\title{
Identification and regulation of (novel) human adipokines : a proteomic approach
}

Citation for published version (APA):

Anja Rosenow, A. R. (2014). Identification and regulation of (novel) human adipokines : a proteomic approach. [Doctoral Thesis, Maastricht University]. Uitgeverij BOXPress.

https://doi.org/10.26481/dis.20140207ar

Document status and date:

Published: 01/01/2014

DOI:

10.26481/dis.20140207ar

Document Version:

Publisher's PDF, also known as Version of record

\section{Please check the document version of this publication:}

- A submitted manuscript is the version of the article upon submission and before peer-review. There can be important differences between the submitted version and the official published version of record.

People interested in the research are advised to contact the author for the final version of the publication, or visit the DOI to the publisher's website.

- The final author version and the galley proof are versions of the publication after peer review.

- The final published version features the final layout of the paper including the volume, issue and page numbers.

Link to publication

\footnotetext{
General rights rights.

- You may freely distribute the URL identifying the publication in the public portal. please follow below link for the End User Agreement:

www.umlib.nl/taverne-license

Take down policy

If you believe that this document breaches copyright please contact us at:

repository@maastrichtuniversity.nl

providing details and we will investigate your claim.
}

Copyright and moral rights for the publications made accessible in the public portal are retained by the authors and/or other copyright owners and it is a condition of accessing publications that users recognise and abide by the legal requirements associated with these

- Users may download and print one copy of any publication from the public portal for the purpose of private study or research.

- You may not further distribute the material or use it for any profit-making activity or commercial gain

If the publication is distributed under the terms of Article $25 \mathrm{fa}$ of the Dutch Copyright Act, indicated by the "Taverne" license above, 
Identification and regulation

$$
\text { of (novel) }
$$

human adipokines

A proteomic approach 


\section{nutúfin}

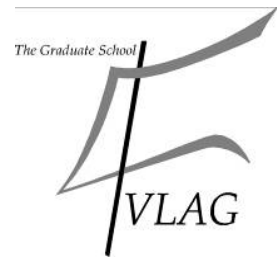

The study presented in this thesis was performed within NUTRIM school for Nutrition, Toxicology and Metabolism which participates in the Graduate School VLAG (Food Technology, Agrobiotechnology, Nutrition and Health Science), accredited by the Royal Netherlands Academy of Arts and Sciences.

$\begin{array}{ll}\text { Cover design: } & \text { sciencephoto.com } \\ \text { Lay-out: } & \text { Anja Rosenow } \\ \text { Printed by: } & \text { Proefschriftmaken.nl/Uitgeverij BOXPress }\end{array}$

(c) Copyright Anja Rosenow, Maastricht 2014

ISBN 978-90-8891-777-6

All right reserved to the author with the exception of those chapters published by the journals referred to. No part of this publications may be produced, stored in a retrieval system, or transmitted, in any form or by any means, without the prior permission in writing from author or the copyright owning journal. 


\title{
Identification and regulation of (novel)
} human adipokines

\author{
A proteomic approach
}

\section{Proefschrift}

ter verkrijging van de graad van doctor

aan de Universiteit Maastricht,

op gezag van de Rector Magnificus,

Prof. Dr. L.L.G. Soete,

volgens het besluit van het College van Decanen,

in het openbaar te verdedigen

op

vrijdag 07 februari 2014

door

\section{Anja Rosenow}

geboren op 09 december 1981,

te Salzwedel, Duitsland 


\section{Promotor}

Prof. dr. Edwin C.M. Mariman

\section{Copromotor}

Dr. Johan Renes

\section{Beoordelingscommissie}

Prof. dr. T. Hackeng (voorzitter)

Prof. dr. M. van Baak

Prof. dr. F. Ramaekers

Prof. dr. M. van Steensel

Studies described in this thesis are financially supported by the School of Life Sciences, transnationale Universiteit Limburg. 


\section{Contents}

Chapter 1

General Introduction

Chapter 2

Novel adipocyte features discovered by adipoproteomics

Chapter 3

Identification of novel human adipocyte secreted proteins by using SGBS cells

Chapter 4

Expression of functional tissue factor by human preadipocytes and adipocytes

Hypoxia-mimetic effects in the secretome of human preadipocytes and adipocytes

Chapter 6

Resveratrol-induced changes of the human adipocyte secretion profile

Chapter 7

Calorie restriction-induced changes in the secretome of human adipocytes, comparison with resveratrol-induced secretome effects

Chapter 8

General Discussion

Summary

Dankwoord

Publications 

Chapter 1

General Introduction 


\section{CHAPTER 1}

\section{Obesity}

Every living cell requires energy to accomplish its function. Mammalian energy sources are carbohydrates, lipids and to a lesser extent proteins which are obtained by food intake. However, in the last decades societal and behavioral changes of human lifestyle have resulted in increased food intake and less physical activity which lead to a positive energy balance. Excess energy is accumulated as body fat in adipose tissue - which results in increased fat mass - and can lead to overweight as well as obesity. Furthermore, the susceptibility to gain weight is also related to genetic factors [1-3].

Overweight is characterized by a body mass index (BMI) of over $25 \mathrm{~kg} / \mathrm{m}^{2}$, a BMI of over 30 $\mathrm{kg} / \mathrm{m}^{2}$ is referred to as obese. Both phenomena have affected humans of all ages, sex, societies and countries, which make overweight and obesity global issues. Furthermore, obesity is associated with premature mortality as well as with several diseases and chronic medical conditions, including skin problems, infertility, stroke, osteoarthritis, obstructive sleep apnea, depression, gastro esophageal reflux disease, hepatic steatosis, gallbladder disease, increased cholesterol and triglyceride levels, increased blood pressure, insulin resistance, type 2 diabetes, dyslipidemia, hypertension, cardiovascular diseases and several cancers such as colon and breast cancer. These health issues and the decreased mobility through increased BMI impair personal life quality and have an enormous impact on national health care costs and the economy as well [4-8]. As such, obesity and its associated complications are one of the major global burdens of our time.

\section{Adipose tissue}

The highly vascularized adipose tissue is a specialized connective tissue that is arranged in a loose association of adipocytes (fat loaded cells) embedded in a construction of collagens. Also stromal-vascular cells including fibroblastic connective tissue cells, leukocytes, macrophages and preadipocytes (precursor cells of the fat loaded adipocytes) are components of adipose tissue. The major weight component of adipose tissue, up to $85 \%$, is lipid that consists of $90-99 \%$ of triglycerides. Other components are free fatty acids, diglycerides, cholesterol, phospholipids, cholesterol esters and monoglycerides. Water, proteins and minerals are the remaining weight components of adipose tissue [9]. Two different forms of adipose tissue are known. The brown adipose tissue (BAT) regulates thermogenesis and white adipose tissue (WAT) regulates energy metabolism and storage.

\section{Brown adipose tissue}

The archetypical BAT has existed for at least 150 million years. However, the BAT is only present in mammals and contains multilocular lipid droplets (one cell contains multiple fat droplets). This tissue, in contrast to the WAT, is located only in specific body regions: 
thyroid/tracheal, mediastinal, paracervical/supraclavicular, parathoracical, supra and perirenal (from the neck region down the air tube) as well as a thin kite-shaped sheet between the shoulder blades in infants. The largest BAT depot has been shown to be the paracervical/supraclavicular (neck) region [10-12]. Approximately $2 \%-5 \%$ of the total body weight is related to the BAT of infants and $0.05 \%-0.01 \%$ is accounted for BAT of adults. This indicates a decrease of BAT with increase of age. In contrast, BAT of mice reaches approximately $5 \%-10 \%$ of their body weight over their whole life period [13].

The main function of BAT is a thermoregulatory thermogenesis by uncoupling the adenosine5 '-triphosphate- (ATP) synthesis from substrate oxidation. The cells of BAT contain many mitochondria which express uncoupling protein 1 (ucp1). Ucp1 is responsible for the proton transport over the inner membrane of the mitochondria that generates heat instead of ATP [14]. Several studies have indicated a metabolic thermogenesis regulation by showing a negative correlation of active BAT and BMI. Moreover, especially young healthy adults have significant cold stimulated active BAT and it seems that women compared to men have a higher amount of active BAT $[10,11,15,16]$. Furthermore, it has been shown that thyroid hormone and catecholamine treatment activate and increase the amount of BAT [17, 18]. These findings indicate the potential of active BAT as a therapeutic approach to obesity and its complications.

\section{White adipose tissue}

The white adipose tissue is mainly localized in subcutaneous (under the skin) or visceral (surrounding inner organ) depots. Compared to subcutaneous fat visceral fat has a higher metabolic activity that may play a major role in causing obesity-related diseases [19, 20]. All different depots have their own characteristics and metabolism, which is shown in different rates of fat accumulation and fat loss.

The majority of WAT is constituted of fat loaded adipocytes, which are organized in unilocular cells (cells with one fat droplet). The formation of white adipose tissue begins before birth and expands rapidly after birth by increased adipocyte numbers (hyperplasia) and/or increased adipocyte size (hypertrophy). Even during adulthood both processes occur. Therefore, hyperplasia and hypertrophy are closely linked to the development of overweight and obesity. However, the development to a mature adipocyte passes several stages starting from an embryonic stem cell precursor with the capacity to differentiate into mesodermal cells like adipocytes, chrondrocytes, osteoblasts and myocytes [21]. Furthermore, the biological process of adipogenesis is supported by hormonal regulation. The most important factors to stimulate the differentiation are: dexamethasone, a glucocorticoid, which stimulates the glycocorticoid receptor pathway, and 3-isobutyl-1-methylxantine (IBMX) that stimulates the cyclic adenosine monophosphate (cAMP)-dependent protein kinase pathway. Insulin, 


\section{CHAPTER 1}

another hormone, is used in high concentration to enhance dexamethasone and IBMX actions. All hormonal stimuli are aimed to activate the PPAR-y and C/EBP- $\alpha$ transcription factors, which induce expression of adipocyte specific genes and thus initiate adipogenesis [21-23].

\section{The Functions of the white adipose tissue}

The major function of WAT adipocytes is the storage of energy and the regulation of the energy balance by tight hormonal control [21]. Storage of energy occurs by the synthesis of triglycerides (TG). This biological process is known as lipogenesis. During lipogenesis, exogenous (dietary) as well as endogenous (de novo synthesized) TG are transported to adipose tissue via lipoproteins. In the capillaries of the adipose tissue TG are hydrolyzed by lipoprotein lipase (LPL) to free fatty acids (FFA) and glycerol. FFA are taken up by the adipocytes while the glycerol is transported to the liver or kidney. There, the glycerol is converted into a dihydroxyacetone phosphate via a glycerol kinase and a glycerol-3phosphate dehydrogenase. In contrast, energy requirements lead to adipocyte TG lipolysis, which results in the hydrolyzation of TG into glycerol and FFA by lipase actions. The hormone-sensitive lipase (HSL) and the adipose triglyceride lipase (ATGL) are responsible for more than $95 \%$ of such lipase activity [24]. As such, HSL is phosphorylated by protein kinase $A(P K A)$ and in turn translocated from the cytoplasm to the lipid droplets where it converts TG into FFA and diacylglycerol in a time-limiting reaction $[25,26]$. In contrast, ATGL is already located on lipid droplets under basal conditions [27], it can work PKAindependently and it is thought to catalyze the initial step of the TG hydrolysis [24, 27]. In addition, ATGL seems to regulate the basal lipolysis in adipocytes while HSL plays a more secondary role [28]. Hydrolyzed FFA are released into the blood circulation, bound to the transport protein serum albumin and are directed to the organ of energy request where FFA are oxidized to $\mathrm{CO}_{2}$ and $\mathrm{H}_{2} \mathrm{O}$ [29]. Within the adipocytes lipogenesis and lipolysis are in balance and are linked to the needs of cellular metabolism. Disturbance of this balance to a higher grade of lipogenesis leads to an increased while a higher grade of lipolysis leads to a decreased fat content. Additional functions of WAT are the thermal isolation, shock cushion of the inner organs as well as the endocrine function [30].

\section{The secretory functions of white adipose tissue}

One of the most important physiological functions of WAT that has a major impact on the human body is the secretion of many endocrine and para/autocrine factors. These influence the homeostasis and metabolism of several organs including heart, liver, lungs, skeletal muscle and the vascular system [22, 31] (Figure 1). Under normal conditions WAT can be described as an endocrine organ, which becomes one of the largest ones during 
overweight/obesity conditions. The paracrine and autocrine actions of adipose tissue are associated with adipogenesis, angiogenesis and remodeling of the adipose tissue while the endocrine actions are linked to energy expenditure, regulation of glucose distribution, food intake and appetite [31-33]. The WAT secreted factors are generally classified as adipokines, although it should be noted that the definition adipokine is not limited to only adipocytesecreted factors. For example, cytokines are mostly secreted by macrophages of the adipose tissue and also classified as adipokines. On the other hand, adipocyte secreted proteins related to the extracellular matrix (ECM) are not classified as adipokines [30, 33]. Therefore, a clear definition of secreted proteins from the adipose tissue is still missing. Among the secreted proteins are hormones, neurotransmitters, cytokines, neuron related proteins, ECM proteins and proteins involved in vascularization, blood flow, lipid and glucose metabolism [34-36].

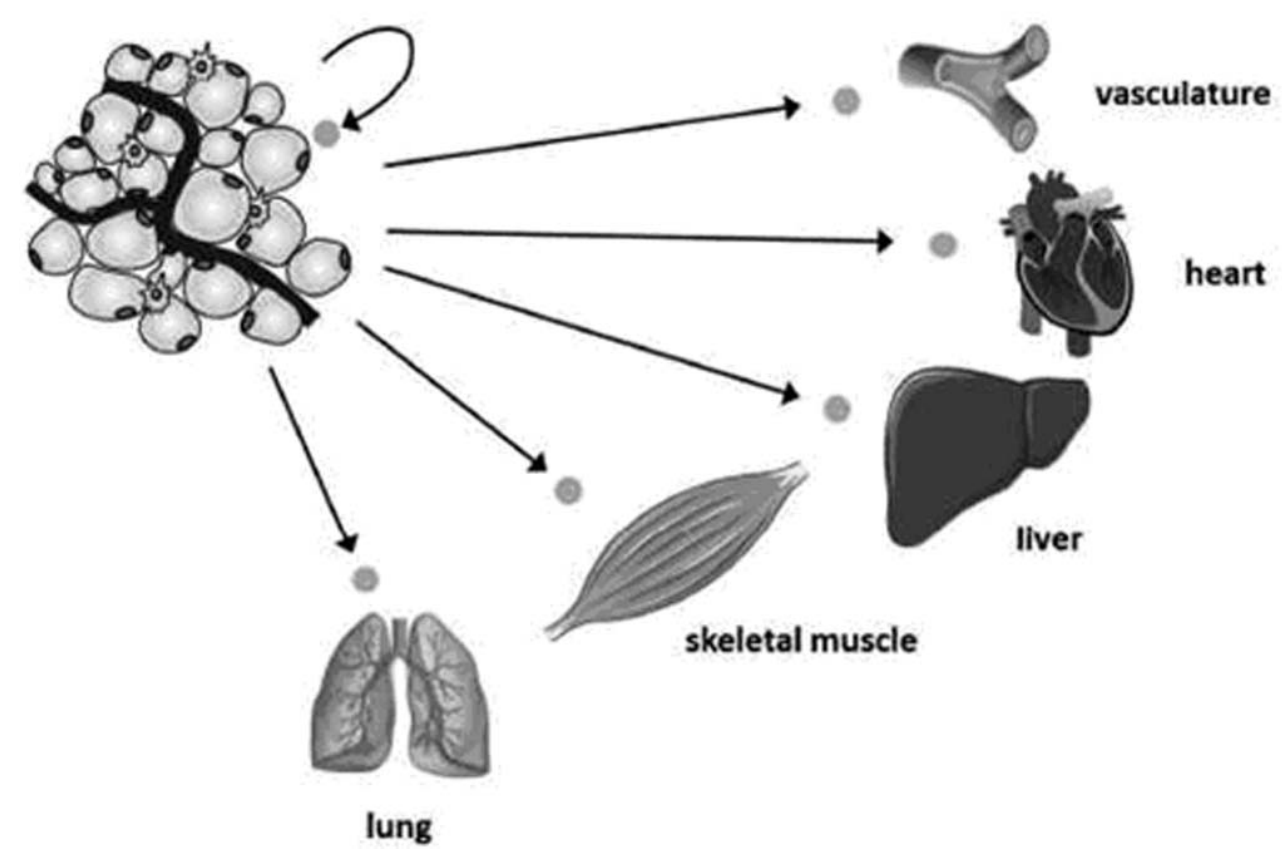

Figure 1: Autocrine/paracrine secretion functions of adipose tissue [37].

As described above, under normal conditions the WAT organization of energy accumulation and utilization is in balance and covers the physiological needs. This is also the case for the WAT secretion function. Disruption of the tightly regulated energy balance leads to changes in the protein secretion pattern [37, 38]. Increased levels of fat loaded adipocytes in an obese state disturb the tightly regulated endocrine function of the WAT to permanently different and secretion profiles. In the last years several rodent [39-44] as well as human [34-36, 45-50] studies have been published that focused on these secretion changes of adipose tissue/adipocytes in which more than 900 different adipocyte-related proteins were identified [37]. The differences in protein expression and regulation are shown over the whole spectrum of adipocyte-related protein classes, which are also related to the ECM, turnover, 


\section{CHAPTER 1}

regulation, signaling and immune regulation. These changes in secretion and circulation patterns of WAT-derived proteins are probably an initiating step for the etiology of obesityassociated metabolic disorders as well as low-grade inflammation [34-36, 39-50]. In addition, compared to normal WAT, obesity-associated WAT may be poorly vascularised, which results in a deficiency of $\mathrm{O}_{2}$ and leads to a hypoxic state within the adipose tissue $[51,52]$. This theory is confirmed by several facts. Firstly, the blood flow to the extended adipose tissue of obese subjects is not elevated compared to lean subjects. Secondly, the blood flow to adipose tissue of obese subjects is not prostprandially increased and thirdly, the diameter of adipocytes in obese subjects is increased up to $180 \mu \mathrm{m}$. The maximum $\mathrm{O}_{2}$ diffusion distance in tissues is around $100 \mu \mathrm{m}$, which may support the development of hypoxia within adipocytes of obese subjects [53-56]. Such hypoxic state may lead to tissue inflammation and cellular dysfunction, which in turn affects secreted factors of the WAT. This can be an additional initiator of a dysregulated secretion profile during obesity [57].

\section{Adipocyte secretion profile}

As has already been mentioned the processes of hyperplasia and hypertrophy lead to an increased amount of adipocytes and to an increased adipocyte cell size. Such processes urge structural remodeling which results in different expression and secretion of structural components including ECM proteins. In addition, reduction of adipocyte intracellular TG content by calorie restriction or therapeutic interventions results in a decreased adipocyte cell size which in turn also induces structural remodeling $[30,58]$. As such, reduced cell size may lead to a decreased expression of cell structure proteins and such mechanical cell shrinking process might be responsible for cellular stress and eventually induced apoptosis [58].

The profile of adipocyte-secreted proteins is broad and several of these proteins are linked to obesity-related metabolic disorders and to the pro-inflammatory state of obese patients. Here some of these are discussed that are relevant for this thesis. These include adiponectin, angiotensinogen, apolipoprotein $E$ (ApoE), haptoglobin, interleukins (IL), leptin, monocyte chemotactic protein (MCP)-1, plasminogen activator inhibitor 1 (PAI-1), pigment epitheliumderived factor (PEDF), prostaglandin-H2 D-isomerase, retinol binding protein (RBP)-4, tumor necrosis factor alpha (TNF- $\alpha$ ), visceral adipose tissue-related serpin (vaspin), visfatin and zinc-alpha-2-glycoprotein (ZAG).

Adiponectin is involved in the energy metabolism and is capable to increase insulin sensitivity. Reduced circulating levels of adiponectin during obesity negatively affects insulin sensitivity that in turn may lead to insulin resistance [59]. Up-regulated adiponectin levels might be beneficial with respect to increased glucose uptake in muscles, insulin sensitivity and suppressed gluconeogenesis in the hepatocytes [60]. Furthermore, adiponectin has also 
been shown to be anti-inflammatory and inhibits pro-inflammatory factors including TNF- $\alpha$ and reactive oxygen species [61].

Angiotensinogen regulates the renin-angiotensin-system and influences blood pressure, body fluids and electrolyte homeostasis [62]. Excess WAT leads to increased circulating levels of angiotensinogen in human and mice [63]. Induced angiotensin expression is related to glucose intolerance and systemic insulin resistance in mice [64].

Angiopoietin-related proteins 1, 2, 3, 4 and 6 regulate angiogenesis while angiopoietinrelated proteins 3, 4 and 6 are also involved in lipid, glucose and energy metabolism [65]. However, angiopoietin-related protein 1 showed phosphatidylinositol 3-kinase/Akt mediated antiapoptotic activities [66].

ApoE regulates TG turnover and the expression of genes which are involved in lipid synthesis to fulfill the energy and lipid homeostasis of cells. Therefore, ApoE is up-regulated during calorie restriction and down-regulated in a high fat environment. An induced expression is associated with increased TG synthesis and TG accumulation as an energy homeostasis protection response [67]. Furthermore, increased signaling of ApoE leads to a conversion of pro-inflammatory M1 macrophages towards M2 macrophages with antiinflammatory phenotype [68].

Haptoglobin is up-regulated during inflammation and obesity. In addition, it is associated with important chemotactic activities towards monocytes [69]. Down-regulation may improve insulin resistance and hepatosteatosis via increased glucose tolerance, insulin sensitivity and increased adiponectin expression [70].

Interleukins (ILs) are related to the group of cytokines. Especially IL-6 and IL-8, which are particularly secreted by visceral adipose tissue, are thought to play an important role in proinflammatory actions and are associated with the initiation of insulin resistance [71, 72].

Leptin is a hormone which interacts with the brain appetite centre and influences the insulin sensitivity. As such, elevated leptin secretion by WAT promotes insulin resistance and in turn increases the risk of leptin resistance [73].

MCP-1 is highly secreted from adipocytes in the obese state and is responsible for recruiting monocytes into adipose tissue. During obesity such recruiting processes are elevated compared to normal conditions and appear to be associated with insulin resistance [74].

PAI-1 initiates fibrinogen formation by matrix metalloproteinase inhibition and fibrinolysis. Increased fibrinogen expression is associated with low-grade inflammation [75]. Downregulation of PAl-1 decreases the chance of development of obesity-induced thrombosis and cardiovascular diseases [75, 76]. Furthermore, down-regulation of PAI-1 shows potential anti-inflammatory effects [77-79].

PEDF secretion levels are associated with enlarged adipose tissue mass and related to insulin resistance [80, 81] and pro-inflammatory action in obese subjects [82]. Down- 


\section{CHAPTER 1}

regulation of PEDF may positively influence the insulin sensitivity and the inflammatory phenotype of obese subjects.

Prostaglandin-H2 D-isomerase (PTGDS) circulating levels are related to adipogenesis. Antisense-mediated decreased levels of PTGDS resulted in increased lipid storage in adipocytes [83]. In addition, PTGDS knock-out mice showed accelerated glucose intolerance [84]. Therefore, PTGDS up-regulation may decrease the risk of glucose intolerance and insulin resistance [83, 84].

RBP-4 is shown to be elevated in serum of insulin resistant patients and is associated with cardiovascular risk factors. As such, RBP-4 is thought to contribute to type 2 diabetes. In addition, RBP-4 inhibits the insulin activity in skeletal muscle and liver [85].

TNF- $\alpha$ has been shown to be an inflammatory cytokine, which is expressed in adipose tissue in the obese state. There is evidence that TNF- $\alpha$ secretion is linked to insulin resistance [86]. Vaspin is an adipokine that is highly expressed and secreted in visceral adipose tissue and is induced by angiogenesis [87]. However, there is no clear correlation between vaspin serum levels and insulin sensitivity or glucose metabolism [88]. It seems that vaspin might be more causative in the development of obesity and obesity-associated disorders and might be considered as a biomarker of these disorders [89].

Visfatin is thought to be highly expressed in visceral adipose tissue. However, its expression profile including its association to obesity [90-92], and its relation to subcutaneous vs. visceral adipose tissue $[90,91,93]$ showed conflicting results. As such, the direct correlation between visfatin and insulin sensitivity is not clear yet [91-93].

ZAG is highly expressed in cancer and adipose tissue. However, ZAG is known to be involved in the lipid metabolism as a lipid-mobilizing factor [94, 95]. It has been shown that ZAG is down-regulated in obesity and is negatively correlated with plasma insulin, C-reactive protein and MCP-1 levels which are associated with obesity-related metabolic disorders [96, 97].

Several adipocyte signaling factors including TNF- $\alpha$, MCP-1 and IL are very low concentrated compared to the whole cell secretome. The identification of such low-abundant proteins is not yet possible with current proteomics technologies, particularly when 2dimensional gel-electrophoresis is applied.

The adipokine resistin is secreted by mice adipocytes while resistin in human adipose tissue is mainly secreted by macrophages [98]. However, in mice as well as in human increased resistin secretion levels are associated with insulin resistance [98].

This overview of a limited set of adipocyte-associated signaling proteins demonstrates the need to further investigate novel adipocyte-related proteins and their relation to obesity and obesity-associated metabolic dysfunction. In addition, the characterization of novel adipocyte-related proteins and their regulation pattern is essential to develop novel treatment 
strategies for obesity and its complications. For the studies described in this thesis proteomics technology has been applied to investigate changes in profiles of adipocytesecreted proteins.

\section{Proteomics}

Proteomics is the study of a complete proteome expressed by a single genome of a cell, tissue or organism. This research direction includes the identification, characterization and quantification of protein isoforms, polymorphisms, modification (alternative splicing and posttranslational), turnover, protein-protein interactions as well as the description of the protein structure and its complexes [29].

To understand single metabolic regulations or the whole physiology of a biological system the study of proteins is essential. Around 25000 human genes [99] are associated with an estimated 500.000 to 1.000 .000 different protein components. This indicates a highly complex and dynamic system, which is internally and externally activated/stimulated [29, $100,101]$. To analyze and interpret such complexity of a single proteome, different strategies are required. The main strategies are: gel-based protein separation with 1-dimensional (1-D) or 2-dimensional gel-electrophoresis (2-DE) combined with mass spectrometry (MS), liquid chromatography (LC) coupled to tandem mass spectrometry (MS/MS), antibody techniques (Western-blotting and antibody array technology), X-ray crystallography, nuclear magnetic resonance spectroscopy and bioinformatics [102, 103]. However, the current proteome technologies are still limited with respect to the sensitivity. Although the detection limits have decreased significantly over the last 10 years, the technology still struggles with the high complexity of proteomes and the high dynamic range (more than $10^{6}$ fold) [101] of protein expression [100, 102].

\section{Gel-approaches combined with mass spectrometry}

With 1-DE the proteins are only separated by molecular masses. As such, complex protein samples are difficult to separate by using 1D approaches due to the low resolution capacity of this technique [102]. Protein separation by 2-DE (separation by isoelectric points and molecular masses) followed by in-gel digestion (e.g. with trypsin) and MALDI-TOF MS significantly enhanced the possibility to analyze complex protein samples and is currently the most classical proteomic approach [104]. The MALDI-TOF MS approach measures the peptide masses as peptide mass fingerprints (PMF) and matches the experimental masses with theoretical masses from a cleavage-site specific database. The 2-DE technique is a semi-quantitative and comparative approach that is capable to visualize simultaneously many different proteins as well as splice variants, post-translational modifications and 


\section{CHAPTER 1}

degraded proteins. However, this approach is time consuming, labor intensive, has a limited dynamic range and visualizes only high abundant proteins. Up to 1000-1500 spots per gel can maximally be visualized from a complex protein sample that may contain over 100000 different proteins. Additionally, proteins with a small $(<10 \mathrm{kDa})$ or a high $(>250 \mathrm{kDa})$ molecular mass as well as acidic $(\mathrm{pl}<3)$ and basic proteins $(\mathrm{pl}>12)$ are difficult to detect with the 2-DE approach. By using sample pre-fractionation, more sensitive staining, larger gel formats or higher resolving gels the 2-DE technique has been improved although not all limitations have been eliminated $[102,105]$. ). Another variant of the 2-DE technique is the differential gel electrophoresis (DIGE). DIGE combines qualitative and quantitative characterization of 2 different samples in one single gel by pre-labeling the proteins with different Cyanine Dyes. It improves the reproducibility but still has the same limitation as the classical 2-DE approach [102, 106].

\section{Mass spectrometry in proteomics research}

To identify and characterize proteins through mass spectrometry the mass to charge $(\mathrm{m} / \mathrm{z})$ ratio of gaseous ions are measured. The mass spectrometer consists of an ion source that produces ions from a peptide sample, a mass analyzer, that analyzes the separated ions, a detector that detects the number of ions and a computer that handles the data by producing a mass spectrum and controlling the instrument $[102,107]$ (Figure 2).

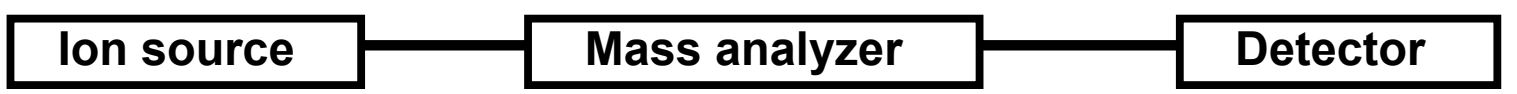

Figure 2: Schematic picture of a mass spectrometry [108].

There are two different techniques to ionize the peptide samples without decomposition and fragmentation - the matrix-assisted desorption ionization (MALDI) [104] and electro spray ionization (ESI). The MALDI technique [104] produces ions out of dry, crystalline matrix of simple peptide samples. The peptide sample is co-crystallized with an excess amount of matrix material (mostly used matrix material: $\alpha$-cyano-4-hydroxycinnamic acid) that absorbs the laser energy. The absorbed energy leads to a desorption of a complex existing of matrix and an intact analytical molecule (a matrix plume) [109] (Figure 3 A). The MALDI ionization process is size and absorption independent. That means MALDI is the perfect tool to identify molecules with a high molecular mass. To analyze more complex samples MALDI can be combined with a HPLC instrument. Compared to the ESI technique, MALDI has a higher tolerance to buffers and salt; it can be used with relatively simple sample preparation, gives rather simple mass spectra and with MALDI it is possible to reanalyze the samples. On the other hand, data obtained from MALDI spectra give less information on a protein compared to ESI spectra $[102,105,107]$. 
The ESI technique uses dissolved samples to form gaseous ions, which are sampled into a vacuum. The use of dissolved peptides allows the coupling of an ESI-equipped mass spectrometer to a HPLC system for pre-separation of the peptide sample. At room temperature ESI produces large, thermally labile, polar molecules, which are ionized without decomposition (Figure $3 \mathrm{~B}$ ). The produced ESI ions depend on the protein molecular mass and structure. Therefore, ESI has the tendency to produce multiply charged ions. Its limitation is the sensitivity for salts and other detergents in buffers. In addition, very complex samples and complicated spectra may reduce the efficacy of this approach [102, 105, 107].

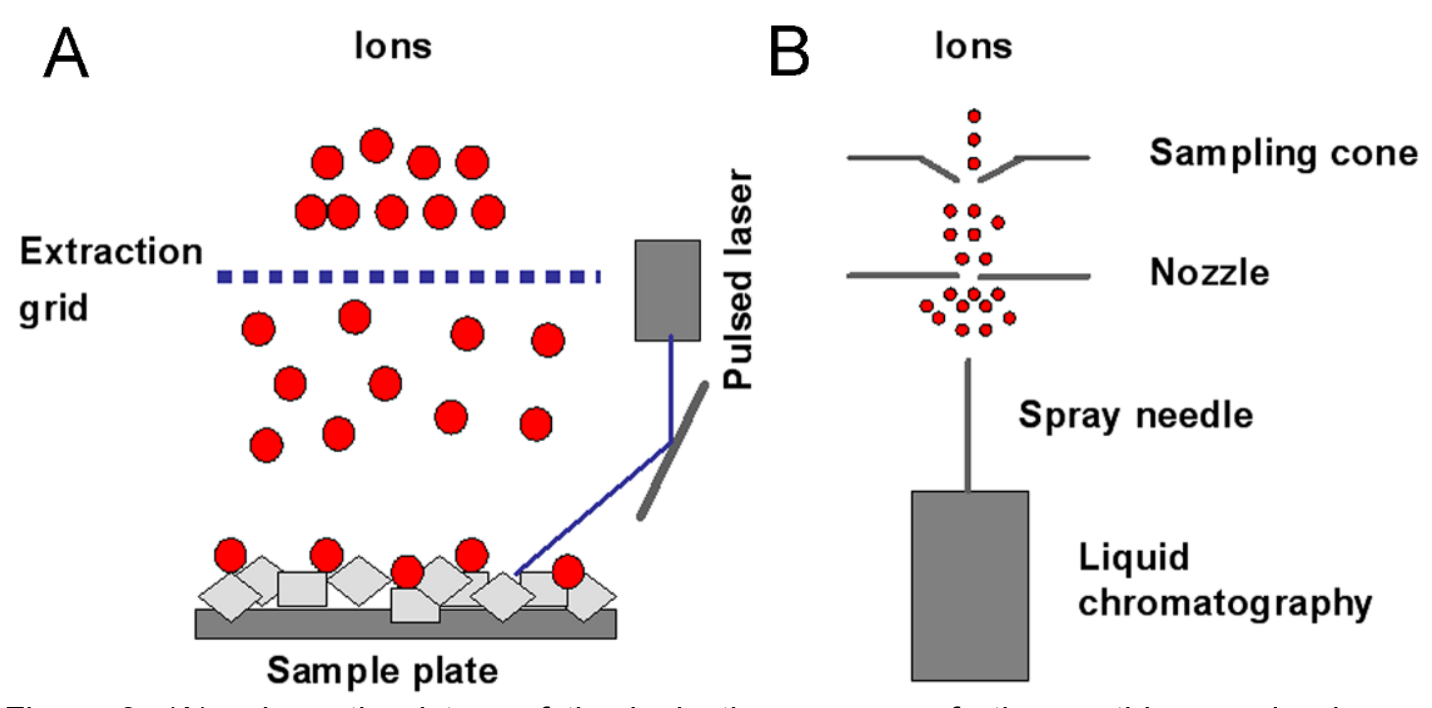

Figure 3: (A) schematic picture of the ionization process of the peptide samples by matrixassociated laser desorption/ionization (MALDI) (left) and (B) by electrospray ionization (ESI) (right ) [108]

\section{Mass analyzer}

Mass resolution, mass accuracy, mass range, sensitivity and the possibility for tandem MS (MS/MS) are important features of mass analyzers. Time-of-flight (TOF), ion trap, quadrupole and Fourier transformation (FT) ion cyclotron resonance (ICR) are the four common mass analyzers, which are used alone or in combination to apply all their advantages. Within the TOF mass analyzer every ion with a different mass takes different time to travel the same distance because of the different accelerating potential of every charged ion. That means, by measuring the time that every ion will take to travel through a field-free region between ion source and detector the $\mathrm{m} / \mathrm{z}$ ratio is determined [110] (Figure $4 \mathrm{~A}$ ). The quadrupole mass analyzer consists of four parallel aligned rods of which two are positively and two are negatively charged. Ions are introduced into this mass analyzer along the central axis of these 4 rods and are filtered based on their $\mathrm{m} / \mathrm{z}$ ratios [111]. As the name indicates an iontrap mass analyzer [112] catches the ions and detects the ions by using their $\mathrm{m} / \mathrm{z}$ ratios. The ion trap is like a quadrupole but forms a closed loop. Therefore an ion trap is also called a quadrupole-ion trap [102]. The FT-MS is a sort of an ion trap that is set up as a cubic cell 


\section{CHAPTER 1}

with a strong magnetic field. This cell consists of three plates: the transmitter, receiver and trapping plate. To analyze the ion masses a constant ion beam that is generated outside the cell is directed to the trap. Ions inside the cells meet a strong magnetic field and start to move in cyclotron motions. The cyclotron motion is characterized by its frequency. This frequency is determined by the magnetic field and the $\mathrm{m} / \mathrm{z}$ ratios of the ions are calculated by Fourier transformation [113] (Figure 4 B). The exact physical workflows of those four mass analyzers are described in the literature [102, 105, 110-113].

A

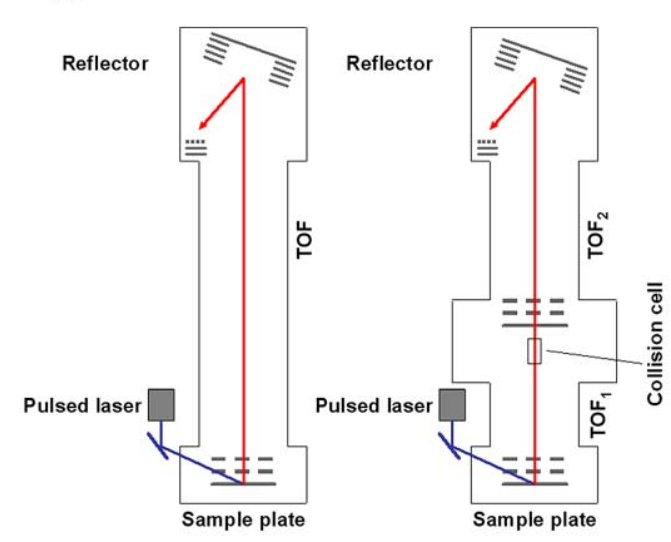

B

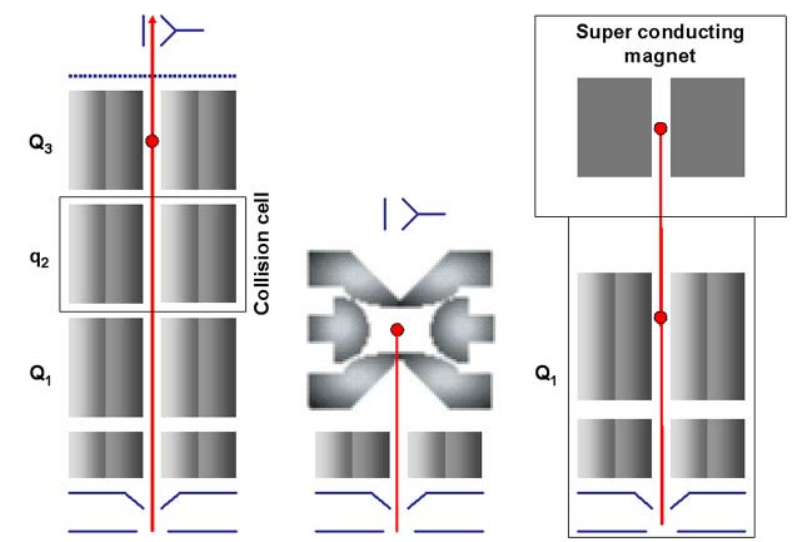

Figure 4: (A) Reflector time-of-flight (TOF) (left) and time-of-flight time-of flight TOF/TOF as mass analyzer of MALDI (right) and (B) triple quadrupole ion trap (linear ion trap), ion trap and Fourier transform ion cyclotron resonance as ESI mass analyzer [108].

\section{Increased performance of MS techniques}

Instruments with two coupled (different) mass analyzers and capacity to further fragment the ionized peptide significantly increased the application of mass spectrometry in proteomics research. With this tandem mass spectrometry approach peptide fragmentation is mediated by an inert gas in a so-called collision chamber placed after the first mass analyzer. In the second mass analyzer the peptide fragments are subsequently measured and MS/MS data are acquired. There are $\mathrm{N}$-terminal and $\mathrm{C}$-terminal peptide fragments that produce $\mathrm{b}$-ions (from N- to C-terminus) and y-ions (C- to N-terminus) [114]. Poor quality spectra, nonhomogeneous fragmentations or poor sequence coverage make protein identification by MS/MS very difficult. The ESI approach is the ideal choice to fragment ionized peptides because ESI produces multi-charged ions. ESI coupled to an ion trap or quadrupole mass analyzer allows high ion selections. Such set-up coupled to pre-separation chromatography like high performance liquid chromatography (HPLC) is a very efficient approach to identify hundreds of proteins in one experiment. This gel-free approach forms the basis of modern mass spectrometry based proteomics [108]. Still this approach is limited with respect to the 
amount of peptides that are fragmented, and, therefore, not all fragmented peptides can be identified. The MALDI technique coupled to a TOF/TOF analyzer combines fragmented ion data with precursor data for more trustworthy identifications. An advantage of tandem mass spectrometry is the capability to identify the proteome of an organism with unknown genome by using the available protein sequencing capacities [108, 115].

Nowadays, 1-DE experiments are often combined with LC-MS/MS approaches. Such gel-LCbased or gel-free LC-MS/MS strategies are among the most efficient proteomic approaches for protein identifications (shot-gun approaches). However, these high through-put approaches miss, in contrast to 2-DE, protein modifications and alternative splicing variants. In addition, quantification of two different protein samples has been another limitation for gelLC-MSMS or gel-free LC-MS/MS strategies. This has been solved by the introduction of stable isotopic labeling strategies that can be used for relative and absolute quantification. The most common labeling strategies are ICAT [116], ITRAQ [117] and SILAC [118]. In addition, with the development of novel reliable computer software label-free quantification strategies that compare peak intensity or volumes are more and more standardized and are increasingly available $[119,120]$ (Figure 5).

Together with the advantage of high through-put measurements, the massive data out-put is at the same time a disadvantage of mass spectrometry-based proteomics. Data collection, analysis, interpretation and visualization and storage require considerable computative power. Furthermore, data publication and sharing ask for higher standardization and transparency. It is necessary to review and validate such data for a more effective electronic search and comparison. To enhance the sensitivity of mass spectrometry and to increase the possibilities to answer new biological questions, further physical improvements like ion source and mass analyzer configurations and new scan mode developments are required.

Although proteomics technologies have still bottlenecks to understand the functional context of a cell, tissue or organism, protein-protein interactions and protein modifications are keyissues that are currently identified, characterized and quantified. Therefore, proteomics, especially mass spectrometry-based proteomics, is one of the most promising research tools to answer many (new) biological questions. These also include the secretome changes of (pre)adipocytes during differentiation and/or different biological conditions including fat overload, hypoxia, calorie restriction and/or drug interventions which are shown here. 


\section{Biological sample \\ (cell, tissue, fluid)}

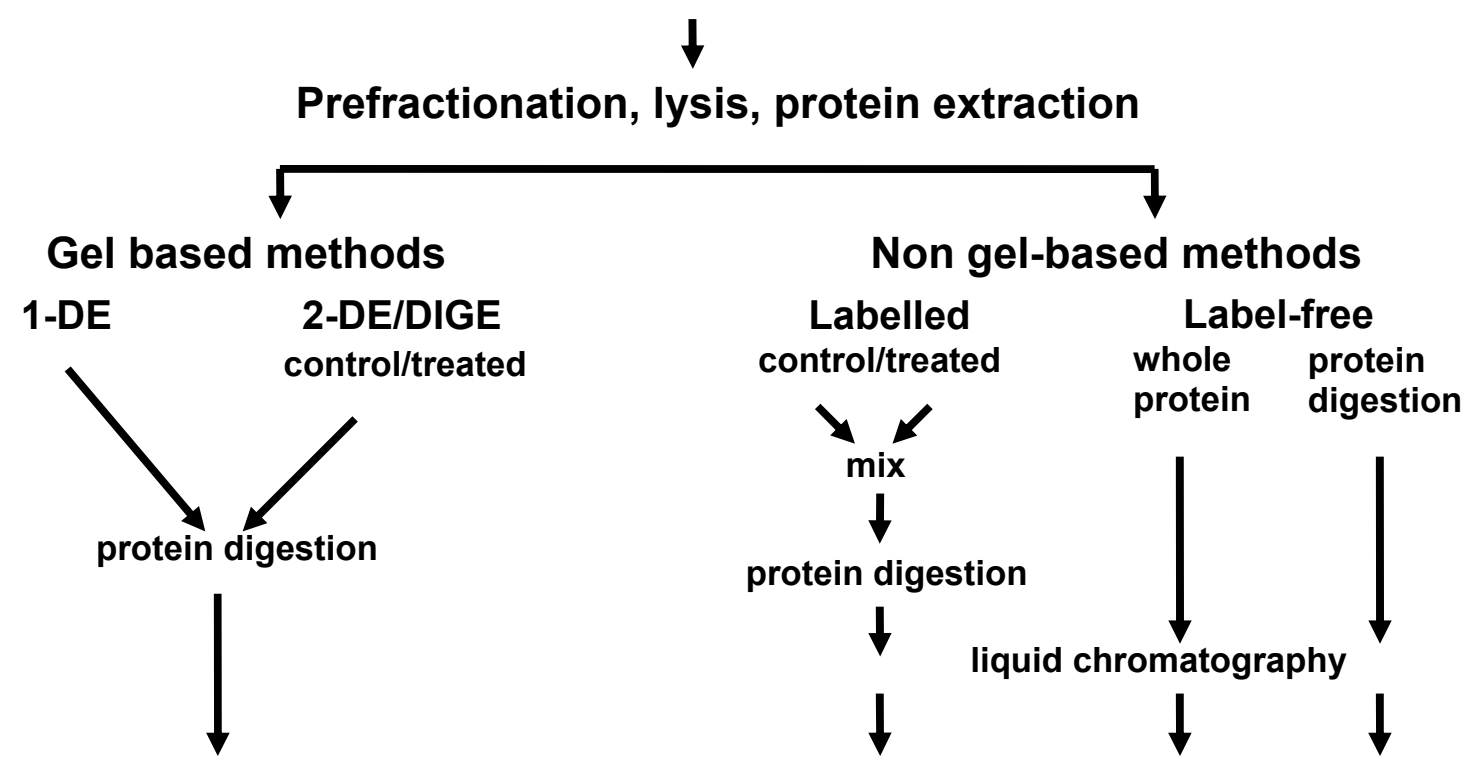

MALDI / ESI mass spectrometry

Figure 5: Overview of the different protein identification approaches.

\section{Outline thesis}

Obesity and its associated diseases currently face epidemic proportions. Therefore, understanding the metabolic regulation of obesity and the beneficial effects of an intervention are important items to prevent obesity and especially its complications. As such, the secretion factors including hormones, cytokines, neuron related proteins, extracellular matrix proteins as well as proteins involved in vascularization, lipid and glucose metabolism of the WAT show autocrine, paracrine and endocrine effects on homeostasis and on metabolism. Differences in their secretion pattern during adipocyte differentiation are thought to initiate obesity-related metabolic and physiological dysfunctions [5, 121]. Therefore, the protein secretion patterns of human (pre)adipocytes were investigated by using proteomics technologies. As study model (pre)adipocytes derived from a subcutaneous fat depot from a Simpson-Golabi-Behmel syndrome (SGBS) patient were used. SGBS cells have been shown to be an ideal human fat cell model because of morphological, functional and biochemical similarities to primary human preadipocytes [122]. Furthermore, SGBS cells have a high differentiation capacity up to $90 \%$ and retain this capacity for at least 30 generations.

Chapter 2 focused on the possibilities of proteomics in the field of adipocyte biology. As such, relevant proteomics technologies and new findings in the context of molecular aspects of adipocyte biology were discussed and highlighted. 
In chapter 3 we investigated the secretion differences of SGBS (pre)adipocytes during adipogenesis. By applying 2-DE-MALDI-MS/MS and nLC-MALDI-MS/MS analysis we have identified a high number of secreted factors as well as additional cellular factors. Comparison of the obtained results with data from studies on different adipocyte strains [34, 35, 40-42, $44,50,123,124]$ confirmed that the SGBS cells with their ideal culturing and manipulation qualities behave as an ideal cell model to study (pre)adipocyte secretion profiles.

Tissue factor (TF), generally known as a blood coagulation factor, is also involved in angiogenesis, an important process in fat tissue expansion during weight gain. Chapter 4 describes a study in which TF expression and activity from SGBS (pre)adipocytes was investigated. Results from this study allowed us to associate TF as a factor for obesityinduced thrombosis and extravascular fibrosis.

Hypoxia is often observed in the fat tissue from obese persons. This prompted us to investigate SGBS (pre)adipocyte secretome changes and protein turnover under hypoxic conditions which is described in chapter $\mathbf{5}$. To mimic hypoxia (pre)adipocytes were treated with $\mathrm{CoCl}_{2}$. 2-DE analysis followed by LC-MS/MS demonstrated differences in secreted protein profiles, while SDS-PAGE followed by MALDI-TOF analysis revealed a turnover profile of hypoxia-stimulated secretion proteins. This investigation allowed us to prove the hypothesis that hypoxia $[51,57,125]$ is connected to adipokine dysregulation as well as WAT dysfunction [126, 127].

Next, we investigated treatment strategies of obesity and its metabolic complications. As such, we studied the effect of resveratrol (RSV) on the SGBS adipocyte secretome (chapter 6). RSV is thought to improve obesity-associated metabolic dysfunction [78, 128]. Therefore, we performed 2-DE followed by LC-MS/MS analysis to investigate the effect of RSV on the secretion profile of mature human SGBS adipocytes. We observed that RSV treatment changed the adipocyte-secretion profile towards a pattern that improves the obesityassociated pro-inflammatory state, insulin sensitivity and thrombotic phenotype.

Another obesity treatment strategy is dietary intervention by calorie reduction (CR). Till now, effects of CR on human adipokine secretion/circulating profiles have not been revealed [129, 130]. As such we investigated the effects of CR on the SGBS adipocyte secretion profile in chapter 7. An extended 2-DE and LC-MS/MS analysis of the secretome allowed us to demonstrate a clear secretion profile related to possible CR-induced beneficial health effects with respect to obesity and obesity-associated disorders. In addition we compared CR- and RSV-induced changes in the secretome profile of human SGBS adipocytes. This study allowed us to look for parallels between both treatments.

Finally, in Chapter 8 the results were discussed in the view of the ideal human (pre)adipocyte model, technical approaches, the secretory function of adipocytes and possible obesity treatment strategies. 


\section{CHAPTER 1}

\section{References}

1. Farooqi, I.S. and S. O'Rahilly, Genetic factors in human obesity. Obes Rev, 2007. 8 Suppl 1: p. 37-40.

2. Rankinen, T., et al., The human obesity gene map: the 2005 update. Obesity (Silver Spring), 2006. 14(4): p. 529-644.

3. Sharma, A.M. and R. Padwal, Obesity is a sign - over-eating is a symptom: an aetiological framework for the assessment and management of obesity. Obes Rev, 2010. 11(5): p. 362-70.

4. Bray, G.A., Medical consequences of obesity. J Clin Endocrinol Metab, 2004. 89(6): p. 2583-9.

5. Dixon, J.B., The effect of obesity on health outcomes. Mol Cell Endocrinol, 2010. 316(2): p. 104-8.

6. Hussain, S.S. and S.R. Bloom, The pharmacological treatment and management of obesity. Postgrad Med, 2011. 123(1): p. 34-44.

7. Runge, C.F., Economic consequences of the obese. Diabetes, 2007. 56(11): p. 266872.

8. Wang, Y., et al., Will all Americans become overweight or obese? estimating the progression and cost of the US obesity epidemic. Obesity (Silver Spring), 2008. 16(10): p. 2323-30.

9. Albright, A.L. and J.S. Stern, Adipose Tissue. Encyclopedia of Sports Medicine and Science; Internet Society for Sport Science: http://sportsci.org, 1998.

10. Cheng, W.Y., Z.H. Zhu, and M. Ouyang, [Patterns and characteristics of brown adipose tissue uptake of 18F-FDG positron emission tomograph/computed tomography imaging]. Zhongguo Yi Xue Ke Xue Yuan Xue Bao, 2009. 31(3): p. 3703.

11. Cypess, A.M., et al., Identification and importance of brown adipose tissue in adult humans. N Engl J Med, 2009. 360(15): p. 1509-17.

12. Heaton, J.M., The distribution of brown adipose tissue in the human. J Anat, 1972. 112(Pt 1): p. 35-9.

13. Enerback, S., Human brown adipose tissue. Cell Metab, 2010. 11(4): p. 248-52.

14. Richard, D. and F. Picard, Brown fat biology and thermogenesis. Front Biosci, 2011. 16: p. $1233-60$.

15. van Marken Lichtenbelt, W.D., et al., Cold-activated brown adipose tissue in healthy men. N Engl J Med, 2009. 360(15): p. 1500-8.

16. Virtanen, K.A., et al., Functional brown adipose tissue in healthy adults. $\mathrm{N}$ Engl $\mathrm{J}$ Med, 2009. 360(15): p. 1518-25. 
17. Lean, M.E., et al., Brown adipose tissue in patients with phaeochromocytoma. Int J Obes, 1986. 10(3): p. 219-27.

18. Skarulis, M.C., et al., Thyroid hormone induced brown adipose tissue and amelioration of diabetes in a patient with extreme insulin resistance. J Clin Endocrinol Metab, 2010. 95(1): p. 256-62.

19. Despres, J.P., et al., Abdominal obesity and the metabolic syndrome: contribution to global cardiometabolic risk. Arterioscler Thromb Vasc Biol, 2008. 28(6): p. 1039-49.

20. Mathieu, P., Abdominal obesity and the metabolic syndrome: a surgeon's perspective. Can J Cardiol, 2008. 24 Suppl D: p. 19D-23D.

21. Gregoire, F.M., C.M. Smas, and H.S. Sul, Understanding adipocyte differentiation. Physiol Rev, 1998. 78(3): p. 783-809.

22. Gregoire, F.M., Adipocyte differentiation: from fibroblast to endocrine cell. Exp Biol Med (Maywood), 2001. 226(11): p. 997-1002.

23. Rosen, E.D. and B.M. Spiegelman, Molecular regulation of adipogenesis. Annu Rev Cell Dev Biol, 2000. 16: p. 145-71.

24. Schweiger, M., et al., Adipose triglyceride lipase and hormone-sensitive lipase are the major enzymes in adipose tissue triacylglycerol catabolism. J Biol Chem, 2006. 281(52): p. 40236-41.

25. Fredrikson, G., et al., Hormone-sensitive lipase of rat adipose tissue. Purification and some properties. J Biol Chem, 1981. 256(12): p. 6311-20.

26. Haemmerle, G., et al., Hormone-sensitive lipase deficiency in mice changes the plasma lipid profile by affecting the tissue-specific expression pattern of lipoprotein lipase in adipose tissue and muscle. J Biol Chem, 2002. 277(15): p. 12946-52.

27. Zimmermann, R., et al., Fat mobilization in adipose tissue is promoted by adipose triglyceride lipase. Science, 2004. 306(5700): p. 1383-6.

28. Miyoshi, H., et al., Adipose triglyceride lipase regulates basal lipolysis and lipid droplet size in adipocytes. J Cell Biochem, 2008. 105(6): p. 1430-6.

29. Voet, D.J. and J.G. Voet, Biochemistry. Vol. 3. 2004: Wiley International Edition. United States

30. Mariman, E.C. and P. Wang, Adipocyte extracellular matrix composition, dynamics and role in obesity. Cell Mol Life Sci, 2010. 67(8): p. 1277-92.

31. Lee, D.E., et al., Getting the message across: mechanisms of physiological cross talk by adipose tissue. Am J Physiol Endocrinol Metab, 2009. 296(6): p. E1210-29.

32. Rosen, E.D. and B.M. Spiegelman, Adipocytes as regulators of energy balance and glucose homeostasis. Nature, 2006. 444(7121): p. 847-53.

33. Wang, P., et al., The secretory function of adipocytes in the physiology of white adipose tissue. J Cell Physiol, 2008. 216(1): p. 3-13. 
34. Alvarez-Llamas, G., et al., Characterization of the human visceral adipose tissue secretome. Mol Cell Proteomics, 2007. 6(4): p. 589-600.

35. Chiellini, C., et al., Characterization of human mesenchymal stem cell secretome at early steps of adipocyte and osteoblast differentiation. BMC Mol Biol, 2008. 9: p. 26.

36. Rosenow, A., et al., Identification of Novel Human Adipocyte Secreted Proteins by Using SGBS Cells. J Proteome Res, 2010. 9: p. 5389-401.

37. Lehr, S., S. Hartwig, and H. Sell, Adipokines: A treasure trove for the discovery of biomarkers for metabolic disorders. Proteomics Clin Appl, 2012. 6(1-2): p. 91-101.

38. Diamanti-Kandarakis, E., et al., The Impact of Endocrine Disruptors on Endocrine Targets. Horm Metab Res, 2010. 42(8): p. 543-52.

39. Aoki, N., et al., Identification and characterization of microvesicles secreted by 3T3L1 adipocytes: redox- and hormone-dependent induction of milk fat globule-epidermal growth factor 8-associated microvesicles. Endocrinology, 2007. 148(8): p. 3850-62.

40. Chen, X., et al., Quantitative proteomic analysis of the secretory proteins from rat adipose cells using a 2D liquid chromatography-MS/MS approach. J Proteome Res, 2005. 4(2): p. 570-7.

41. Kratchmarova, I., et al., A proteomic approach for identification of secreted proteins during the differentiation of 3T3-L1 preadipocytes to adipocytes. Mol Cell Proteomics, 2002. 1(3): p. 213-22.

42. Molina, H., et al., Temporal profiling of the adipocyte proteome during differentiation using a five-plex SILAC based strategy. J Proteome Res, 2009. 8(1): p. 48-58.

43. Roca-Rivada, A., et al., Secretome analysis of rat adipose tissues shows locationspecific roles for each depot type. J Proteomics, 2011. 74(7): p. 1068-79.

44. Wang, P., et al., Profiling of the secreted proteins during 3T3-L1 adipocyte differentiation leads to the identification of novel adipokines. Cell Mol Life Sci, 2004. 61(18): p. 2405-17.

45. Celis, J.E., et al., Identification of extracellular and intracellular signaling components of the mammary adipose tissue and its interstitial fluid in high risk breast cancer patients: toward dissecting the molecular circuitry of epithelial-adipocyte stromal cell interactions. Mol Cell Proteomics, 2005. 4(4): p. 492-522.

46. Kim, J., et al., Comparative analysis of the secretory proteome of human adipose stromal vascular fraction cells during adipogenesis. Proteomics, 2010. 10(3): p. 394405.

47. Lehr, S., et al., Identification and validation of novel adipokines released from primary human adipocytes. Mol Cell Proteomics, 2012. 11(1): p. M111 010504. 
48. Roelofsen, $\mathrm{H}_{\text {., }}$ et al., Comparison of isotope-labeled amino acid incorporation rates (CILAIR) provides a quantitative method to study tissue secretomes. Mol Cell Proteomics, 2009. 8(2): p. 316-24.

49. Zhong, J., et al., Temporal profiling of the secretome during adipogenesis in humans. J Proteome Res, 2010. 9(10): p. 5228-38.

50. Zvonic, S., et al., Secretome of primary cultures of human adipose-derived stem cells: modulation of serpins by adipogenesis. Mol Cell Proteomics, 2007. 6(1): p. 18-28.

51. Fleischmann, E., et al., Tissue oxygenation in obese and non-obese patients during laparoscopy. Obes Surg, 2005. 15(6): p. 813-9.

52. Stuart Wood, I., et al., Cellular hypoxia and adipose tissue dysfunction in obesity. Proc Nutr Soc, 2009. 68(4): p. 370-7.

53. Brahimi-Horn, M.C. and J. Pouyssegur, Oxygen, a source of life and stress. FEBS Lett, 2007. 581(19): p. 3582-91.

54. Dimitriadis, G., et al., Impaired postprandial blood flow in adipose tissue may be an early marker of insulin resistance in type 2 diabetes. Diabetes Care, 2007. 30(12): p. 3128-30.

55. Karpe, F., et al., Impaired postprandial adipose tissue blood flow response is related to aspects of insulin sensitivity. Diabetes, 2002. 51(8): p. 2467-73.

56. Skurk, T., et al., Relationship between adipocyte size and adipokine expression and secretion. J Clin Endocrinol Metab, 2007. 92(3): p. 1023-33.

57. Trayhurn, P. and I.S. Wood, Adipokines: inflammation and the pleiotropic role of white adipose tissue. Br J Nutr, 2004. 92(3): p. 347-55.

58. Mariman, E.C., An adipobiological model for weight regain after weight loss. Adipobiology 2011. 3.

59. Kadowaki, T., et al., Adiponectin and adiponectin receptors in insulin resistance, diabetes, and the metabolic syndrome. J Clin Invest, 2006. 116(7): p. 1784-92.

60. Sharma, A.M. and V.T. Chetty, Obesity, hypertension and insulin resistance. Acta Diabetol, 2005. 42 Suppl 1: p. S3-8.

61. Mandal, P., et al., The anti-inflammatory effects of adiponectin are mediated via a heme oxygenase-1-dependent pathway in rat Kupffer cells. Hepatology, 2010. 51(4): p. 1420-9.

62. Schmieder, R.E., et al., Renin-angiotensin system and cardiovascular risk. Lancet, 2007. 369(9568): p. 1208-19.

63. Yasue, S., et al., Adipose tissue-specific regulation of angiotensinogen in obese humans and mice: impact of nutritional status and adipocyte hypertrophy. Am $\mathrm{J}$ Hypertens, 2010. 23(4): p. 425-31. 
64. Kalupahana, N.S., et al., Overproduction of angiotensinogen from adipose tissue induces adipose inflammation, glucose intolerance, and insulin resistance. Obesity (Silver Spring), 2012. 20(1): p. 48-56.

65. Hato, T., M. Tabata, and Y. Oike, The role of angiopoietin-like proteins in angiogenesis and metabolism. Trends Cardiovasc Med, 2008. 18(1): p. 6-14.

66. Kubota, Y., et al., Cooperative interaction of Angiopoietin-like proteins 1 and 2 in zebrafish vascular development. Proc Natl Acad Sci U S A, 2005. 102(38): p. 135027.

67. Huang, Z.H., et al., Nutritional regulation of adipose tissue apolipoprotein E expression. Am J Physiol Endocrinol Metab, 2007. 293(1): p. E203-9.

68. Baitsch, D., et al., Apolipoprotein $E$ induces antiinflammatory phenotype in macrophages. Arterioscler Thromb Vasc Biol, 2011. 31(5): p. 1160-8.

69. Maffei, M., et al., The obesity and inflammatory marker haptoglobin attracts monocytes via interaction with chemokine (C-C motif) receptor 2 (CCR2). BMC Biol, 2009. 7: p. 87.

70. Lisi, S., et al., Obesity-associated hepatosteatosis and impairment of glucose homeostasis are attenuated by haptoglobin deficiency. Diabetes, 2011. 60(10): p. 2496-505.

71. Bruun, J.M., et al., Association between measures of insulin sensitivity and circulating levels of interleukin-8, interleukin-6 and tumor necrosis factor-alpha. Effect of weight loss in obese men. Eur J Endocrinol, 2003. 148(5): p. 535-42.

72. Fain, J.N., Release of interleukins and other inflammatory cytokines by human adipose tissue is enhanced in obesity and primarily due to the nonfat cells. Vitam Horm, 2006. 74: p. 443-77.

73. Seufert, J., Leptin effects on pancreatic beta-cell gene expression and function. Diabetes, 2004. 53 Suppl 1: p. S152-8.

74. Kanda, H., et al., MCP-1 contributes to macrophage infiltration into adipose tissue, insulin resistance, and hepatic steatosis in obesity. J Clin Invest, 2006. 116(6): p. 1494-505.

75. Nieuwdorp, M., et al., Hypercoagulability in the metabolic syndrome. Curr Opin Pharmacol, 2005. 5(2): p. 155-9.

76. Skurk, T. and H. Hauner, Obesity and impaired fibrinolysis: role of adipose production of plasminogen activator inhibitor-1. Int J Obes Relat Metab Disord, 2004. 28(11): p. 1357-64.

77. Ahn, J., et al., Resveratrol inhibits TNF-alpha-induced changes of adipokines in 3T3L1 adipocytes. Biochem Biophys Res Commun, 2007. 364(4): p. 972-7. 
78. Olholm, J., et al., Anti-inflammatory effect of resveratrol on adipokine expression and secretion in human adipose tissue explants. Int J Obes (Lond), 2010. 34(10): p. 154653.

79. Yen, G.C., et al., Effects of polyphenolic compounds on tumor necrosis factor-alpha (TNF-alpha)-induced changes of adipokines and oxidative stress in 3T3-L1 adipocytes. J Agric Food Chem, 2011. 59(2): p. 546-51.

80. Wang, P., et al., Plasma pigment epithelium-derived factor is positively associated with obesity in Caucasian subjects, in particular with the visceral fat depot. Eur $\mathrm{J}$ Endocrinol, 2008. 159(6): p. 713-8.

81. Borg, M.L., et al., Pigment epithelium-derived factor regulates lipid metabolism via adipose triglyceride lipase. Diabetes, 2011. 60(5): p. 1458-66.

82. Famulla, S., et al., Pigment epithelium-derived factor (PEDF) is one of the most abundant proteins secreted by human adipocytes and induces insulin resistance and inflammatory signaling in muscle and fat cells. Int J Obes (Lond), 2011. 35(6): p. 76272.

83. Chowdhury, A.A., et al., Sustained expression of lipocalin-type prostaglandin D synthase in the antisense direction positively regulates adipogenesis in cloned cultured preadipocytes. Biochem Biophys Res Commun, 2011. 411(2): p. 287-92.

84. Ragolia, L., et al., Accelerated glucose intolerance, nephropathy, and atherosclerosis in prostaglandin D2 synthase knock-out mice. J Biol Chem, 2005. 280(33): p. 2994655.

85. Yang, Q., et al., Serum retinol binding protein 4 contributes to insulin resistance in obesity and type 2 diabetes. Nature, 2005. 436(7049): p. 356-62.

86. Hotamisligil, G.S., Mechanisms of TNF-alpha-induced insulin resistance. Exp Clin Endocrinol Diabetes, 1999. 107(2): p. 119-25.

87. Hida, K., et al., Visceral adipose tissue-derived serine protease inhibitor: a unique insulin-sensitizing adipocytokine in obesity. Proc Natl Acad Sci U S A, 2005. 102(30): p. 10610-5.

88. Youn, B.S., et al., Serum vaspin concentrations in human obesity and type 2 diabetes. Diabetes, 2008. 57(2): p. 372-7.

89. Li, Q., et al., A novel adipocytokine, visceral adipose tissue-derived serine protease inhibitor (vaspin), and obesity. J Int Med Res, 2008. 36(4): p. 625-9.

90. Berndt, J., et al., Plasma visfatin concentrations and fat depot-specific mRNA expression in humans. Diabetes, 2005. 54(10): p. 2911-6.

91. Pagano, C., et al., Reduced plasma visfatin/pre-B cell colony-enhancing factor in obesity is not related to insulin resistance in humans. J Clin Endocrinol Metab, 2006. 91(8): p. 3165-70. 


\section{CHAPTER 1}

92. Chen, M.P., et al., Elevated plasma level of visfatin/pre-B cell colony-enhancing factor in patients with type 2 diabetes mellitus. J Clin Endocrinol Metab, 2006. 91(1): p. 2959.

93. Varma, V., et al., Human visfatin expression: relationship to insulin sensitivity, intramyocellular lipids, and inflammation. J Clin Endocrinol Metab, 2007. 92(2): p. 666-72.

94. Hassan, M.I., et al., Zinc alpha 2-glycoprotein: a multidisciplinary protein. Mol Cancer Res, 2008. 6(6): p. 892-906.

95. Bing, C., et al., Zinc-alpha2-glycoprotein, a lipid mobilizing factor, is expressed in adipocytes and is up-regulated in mice with cancer cachexia. Proc Natl Acad Sci U S A, 2004. 101(8): p. 2500-5.

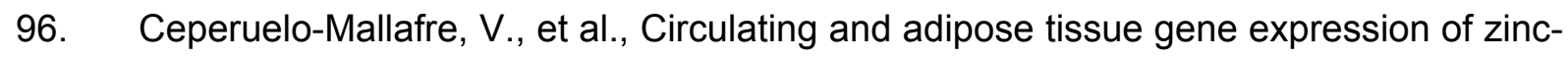
alpha2-glycoprotein in obesity: its relationship with adipokine and lipolytic gene markers in subcutaneous and visceral fat. J Clin Endocrinol Metab, 2009. 94(12): p. 5062-9.

97. Mracek, T., et al., The adipokine zinc-alpha2-glycoprotein (ZAG) is downregulated with fat mass expansion in obesity. Clin Endocrinol (Oxf), 2010. 72(3): p. 334-41.

98. Arner, P., Resistin: yet another adipokine tells us that men are not mice. Diabetologia, 2005. 48(11): p. 2203-5.

99. Pearson, H., Geneticists play the numbers game in vain. Nature, 2003. 423(6940): p. 576.

100. Lundberg, E., et al., Defining the transcriptome and proteome in three functionally different human cell lines. Mol Syst Biol, 2010. 6: p. 450.

101. Tyers, M. and M. Mann, From genomics to proteomics. Nature, 2003. 422(6928): p. 193-7.

102. Lane, C.S., Mass spectrometry-based proteomics in the life sciences. Cell Mol Life Sci, 2005. 62(7-8): p. 848-69.

103. Pandey, A. and M. Mann, Proteomics to study genes and genomes. Nature, 2000. 405(6788): p. 837-46.

104. Karas, M. and F. Hillenkamp, Laser desorption ionization of proteins with molecular masses exceeding 10,000 daltons. Anal Chem, 1988. 60(20): p. 2299-301.

105. Canas, B., et al., Mass spectrometry technologies for proteomics. Brief Funct Genomic Proteomic, 2006. 4(4): p. 295-320.

106. Marouga, R., S. David, and E. Hawkins, The development of the DIGE system: 2D fluorescence difference gel analysis technology. Anal Bioanal Chem, 2005. 382(3): p. 669-78. 
107. Haselberg, R., G.J. de Jong, and G.W. Somsen, Capillary electrophoresis-mass spectrometry for the analysis of intact proteins. J Chromatogr A, 2007. 1159(1-2): p. 81-109.

108. Aebersold, R. and M. Mann, Mass spectrometry-based proteomics. Nature, 2003. 422(6928): p. 198-207.

109. Karas, M., M. Gluckmann, and J. Schafer, Ionization in matrix-assisted laser desorption/ionization: singly charged molecular ions are the lucky survivors. J Mass Spectrom, 2000. 35(1): p. 1-12.

110. Weickhardt, C., F. Moritz, and J. Grotemeyer, Time-Of-Flight Mass Spectrometry: State-of-the-Art in Chemical Analysis and Molecular Science. Mass Spectrometry Reviews, 1996. 15: p. 139-162.

111. Steel, C. and M. Henchman, Understanding the quadrupole mass filter through computer stimulation. J Chemical Edu 1998. 75: p. 1049-54.

112. Guan, Z., et al., Real-time monitoring of the gas phase reactions of a single ion population using the remeasurement experiment in Fourier transform ion cyclotron resonance mass spectrometry. Anal Chem, 1995. 67(8): p. 1453-8.

113. Marshall, A.G., C.L. Hendrickson, and G.S. Jackson, Fourier transform ion cyclotron resonance mass spectrometry: a primer. Mass Spectrom Rev, 1998. 17(1): p. 1-35.

114. Biemann, K., Mass spectrometry of peptides and proteins. Annu Rev Biochem, 1992. 61: p. $977-1010$

115. Cotter, R.J., W. Griffith, and C. Jelinek, Tandem time-of-flight (TOF/TOF) mass spectrometry and the curved-field reflectron. J Chromatogr B Analyt Technol Biomed Life Sci, 2007. 855(1): p. 2-13.

116. Gygi, S.P., et al., Quantitative analysis of complex protein mixtures using isotopecoded affinity tags. Nat Biotechnol, 1999. 17(10): p. 994-9.

117. Ross, P.L., et al., Multiplexed protein quantitation in Saccharomyces cerevisiae using amine-reactive isobaric tagging reagents. Mol Cell Proteomics, 2004. 3(12): p. 115469.

118. Mann, M., Functional and quantitative proteomics using SILAC. Nat Rev Mol Cell Biol, 2006. 7(12): p. 952-8.

119. Beck, F., et al., The good, the bad, the ugly: Validating the mass spectrometric analysis of modified peptides. Proteomics, 2011. 11(6): p. 1099-109.

120. Hansson, J., et al., Time-resolved quantitative proteome analysis of in vivo intestinal development. Mol Cell Proteomics, 2011. 10(3): p. M110 005231.

121. Goossens, G.H., The role of adipose tissue dysfunction in the pathogenesis of obesity-related insulin resistance. Physiol Behav, 2008. 94(2): p. 206-18. 


\section{CHAPTER 1}

122. Wabitsch, M., et al., Characterization of a human preadipocyte cell strain with high capacity for adipose differentiation. Int J Obes Relat Metab Disord, 2001. 25(1): p. 815.

123. Lim, J.M., et al., Defining the regulated secreted proteome of rodent adipocytes upon the induction of insulin resistance. J Proteome Res, 2008. 7(3): p. 1251-63.

124. Sell, H., et al., Cytokine secretion by human adipocytes is differentially regulated by adiponectin, AICAR, and troglitazone. Biochem Biophys Res Commun, 2006. 343(3): p. $700-6$.

125. Trayhurn, P., B. Wang, and I.S. Wood, Hypoxia in adipose tissue: a basis for the dysregulation of tissue function in obesity? Br J Nutr, 2008. 100(2): p. 227-35.

126. Halberg, N., et al., Hypoxia-inducible factor 1alpha induces fibrosis and insulin resistance in white adipose tissue. Mol Cell Biol, 2009. 29(16): p. 4467-83.

127. Khan, T., et al., Metabolic dysregulation and adipose tissue fibrosis: role of collagen VI. Mol Cell Biol, 2009. 29(6): p. 1575-91.

128. Timmers, S., et al., Calorie Restriction-like Effects of 30 Days of Resveratrol Supplementation on Energy Metabolism and Metabolic Profile in Obese Humans. Cell Metab, 2011. 14(5): p. 612-22.

129. Klempel, M.C. and K.A. Varady, Reliability of leptin, but not adiponectin, as a biomarker for diet-induced weight loss in humans. Nutr Rev, 2011. 69(3): p. 145-54.

130. Varady, K.A., et al., Degree of weight loss required to improve adipokine concentrations and decrease fat cell size in severely obese women. Metabolism, 2009. 58(8): p. 1096-101. 


\section{Chapter 2}

\section{Novel adipocyte features discovered by adipoproteomics}

Adapted from: Johan Renes, Anja Rosenow and Edwin Mariman

Adipobiology 2009; 1: 7-18 


\begin{abstract}
Obesity and its associated complications will be the most important near-future medical burden in Western-type societies. One hallmark of obesity is the differentiation of preadipocytes into mature fat-loaded adipocytes present in subcutaneous and visceral fat depots. Furthermore, (pre)adipocytes secrete proteins, known as adipokines, with changing profiles during fat accumulation. Adipocytes serve an important function with respect to energy homeostasis, body insulation and organ protection. Adipocyte dysfunction results in the initiation and progression of obesity-associated disorders. Obviously, knowledge of the adipocyte behaviour under different nutritional conditions and the cross-talk of adipocytes with other cells and organs are key issues to develop proper intervention strategies. A full understanding of the adipocyte behaviour requires a systems biology approach with integrated transcriptomics, proteomics and metabolomics data. This review focuses on the contribution of proteomics research in adipocyte biology. Proteome studies on adipocytes exist for almost 30 years but are boosted in the last decade with the enormous technological developments in mass spectrometry technology. The relevance of proteomics technologies in understanding molecular aspects of adipocyte biology is discussed. Recent novel findings and particularly the identification of novel adipokines are highlighted.
\end{abstract}




\section{Introduction}

Adipocytes play an essential role in normal physiology with respect to energy balance, glucose homeostasis, heat regulation and organ protection. Furthermore, adipocytes are involved in the immune response, blood pressure control, haemostasis, bone mass and thyroid and reproductive function [1, 2]. A deregulation of the adipocyte function results in metabolic dysfunction of the body and the development of obesity-associated diseases like type 2 diabetes, non-alcoholic steatohepatitis, cardiovascular diseases and cancer [3, 4]. With the expected increase in the global overweight population (WHO, Fact sheet on obesity and overweight, September 2006, http://www.who.int/topics/obesity/en/), the number of people that suffer from obesity-associated diseases will consequently rise too [5-7]. Understanding the molecular events leading to adipocyte dysfunction and the related metabolic complications is thus required to provide targets for appropriate intervention to reduce the upcoming metabolic disease burden. During the last decades genomics technologies provided further insights regarding the molecular events underlying the development of obesity and related diseases. Here the contribution of proteomics research with respect to adipocyte biology is highlighted. It is beyond the scope of this review to describe each adipocyte protein that has been identified instead novel biological features of adipocytes discovered by proteomics are discussed.

\section{The proteome: more than just the protein complement of the genome}

The molecular events during preadipocyte differentiation have been examined in a broader sense by several large-scale gene analysis studies [8-10]. Although transcriptomics data provide important information about transcription rates and mRNA turnover, knowledge about protein expression, post-translational modifications and protein turnover, essential for cellular physiology, cannot be covered by this technology. In addition, the correlation between mRNA expression levels and the expression levels of corresponding protein products is low in mammalian systems [11]. For instance, we previously showed that insulin stimulated protein secretion from murine 3T3-L1 adipocytes without increasing the mRNA levels of the corresponding genes [12]. Together, additional monitoring of the cellular protein complement, the proteome, is required to further understand the adipocyte behaviour. The importance of this issue is reflected by the overwhelming amount of different proteins that can be produced by a single genome due to alternative splicing and post-translational modifications. In contrast to the genome, the proteome is highly dynamic that differs in time and in response to external factors [13]. Furthermore, expression and function of many proteins are modified through interactions with other proteins such as kinases and proteases. In fact, most biological systems are controlled by complex protein-protein interaction networks [14]. 


\section{CHAPTER 2}

Consequently, detailed proteome studies may provide additional valuable information about the (patho) physiology of an organism. The dynamic nature of the proteome can be a pitfall for reproducible results, as such; proteome studies require careful experimentation under well-defined conditions.

\section{Proteome analysis strategies}

It is currently not possible to analyze the total proteome of an organism at once. This is due to i) extreme complexity of the proteome, ii) the dynamic nature of the proteome, iii) limitations of the analysis techniques to cover the orders of magnitude difference in protein expression levels and $i v$ ) the differences in physical properties of the proteins. Three main strategies that are currently applied in large-scale proteome research are i) gel-based protein separation, either by 1- or 2-dimensional electrophoresis (2-DE), combined with mass spectrometry $[15,16]$ ii) liquid chromatography coupled to tandem mass spectrometry (LCMSMS) $[17,18]$ and iii) antibody array technology [19, 20], see Figure 1. 


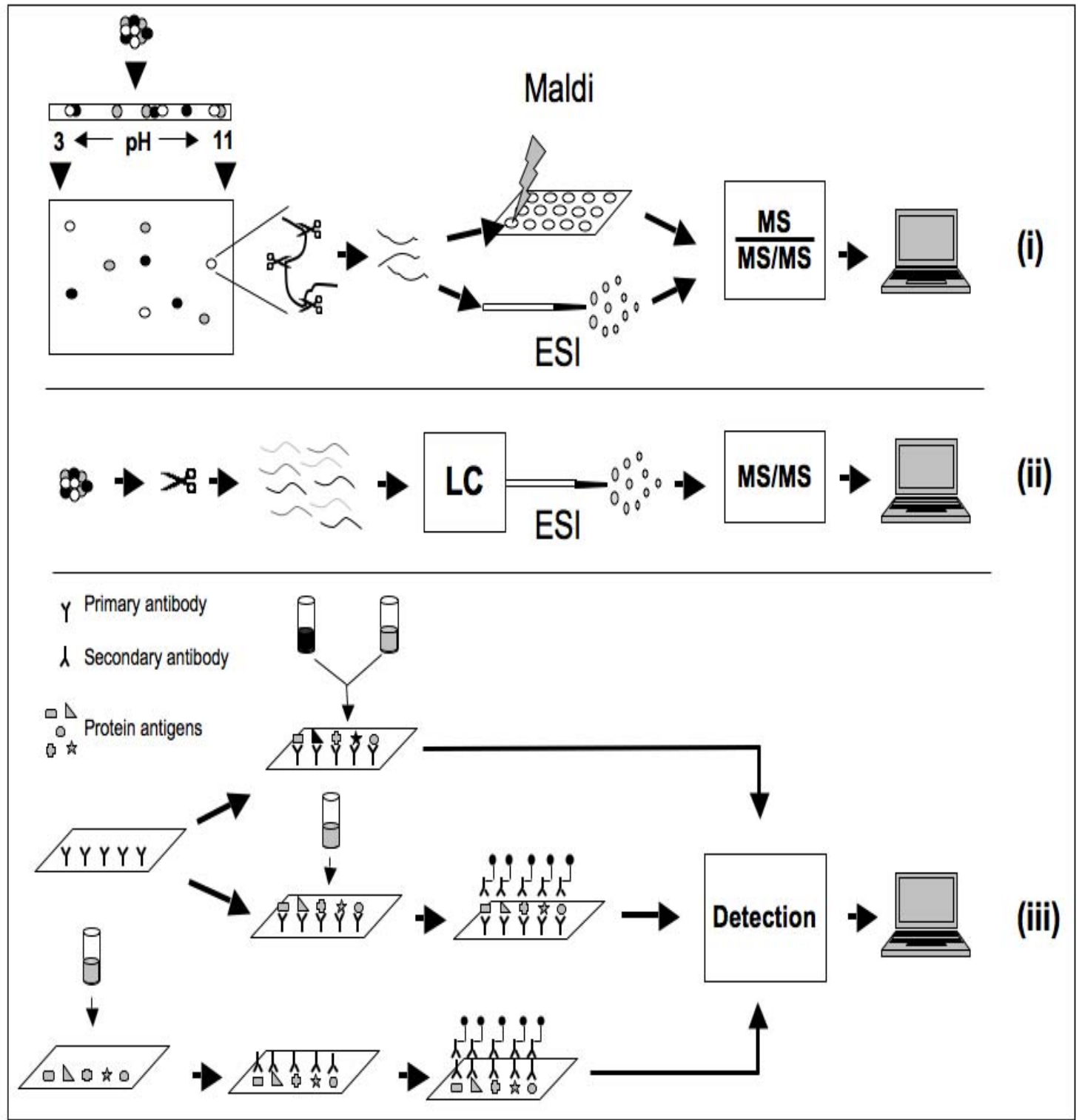

Figure 1: Current strategies in large-scale proteomics studies. (i) Proteomics by 2-DE combined with mass spectrometry. With 2-DE a protein sample is first separated by iso-electric focussing on an immobilized $\mathrm{pH}$ gradient (max. $\mathrm{pH} 3-11$ ) according to the iso-electric points of the proteins. Subsequent separation is based on the molecular mass of the proteins by using sodium dodecyl sulphate polyacrylamide gel electrophoresis. Visualization of the protein spots occurs by staining the gel with silver nitrate, colloidal stains like Coomassie Brilliant Blue or fluorescent dyes. Gels containing different samples are compared by imaging software and differentially expressed protein spots are excised. For identification, proteins are digested (usually by trypsin) and dependent on the type of instrument the peptides can directly be introduced into the mass spectrometer by electrospray ionisation (ESI) or spotted on a target plate for matrix assisted laser desorption ionisation (MALDI) mass spectrometry (see ref. 15 and 16 for details). With the DIGE technology a similar strategy is applied. (ii) LCMSMS-mediated proteomics. In contrast to the gel-based approach, this procedure starts with digestion of the protein sample. Peptides are subsequently separated by liquid chromatography (LC). Separated peptides are directly introduced into the mass spectrometer by electrospray ionisation (ESI). An alternative to this on-line LCMSMS approach is an off-line method where the separated peptides are automatically spotted on a MALDI target plate for subsequent MSMS- identification. (iii) Antibody array technology. Three formats of this technology are displayed (see ref. 19 and 20 for details). A commonly used method is pre-labelling of the proteins samples, which are subsequently mixed and applied to glass slides pre-spotted with a set of available antibodies. Detection of the captured fluorescent antigens occurs by laser scanning. Second, unlabelled protein samples can be applied to similar antibody slides after which the captured antigens are detected by a cocktail of labelled secondary antibodies matched to the pre-spotted ones. The third method involves spotting of the protein sample antigens on the slide. Subsequent detection occurs by labelled read-out antibodies. 


\section{Initial proteome studies on adipocyte biology}

Already in 1979 a 2-DE proteome study was conducted on 3T3-L1 differentiation [32]. Changes in the biosynthesis of 30 cytoplasmic, 9 non-histone chromosome-associated proteins and 24 membrane proteins were found. Unfortunately, only 1 protein could be tentatively identified as actin. Despite that none of the proteins was sequenced, remaining the identity of the proteins unknown, this study demonstrated for the first time that synthesis of several proteins was altered during 3T3-L1 differentiation. Subsequent studies confirmed these results by showing increased $\left[{ }^{3} \mathrm{H}\right]$-leucine incorporation into cellular protein extracts of differentiating 3T3-L1 cells [33] and an increased biosynthesis of glycolytic enzymes in 3T3F442 cells [34]. However, large-scale proteomics studies, including detailed protein identification, on adipocyte biology could only be conducted after the implementation of mass spectrometry in protein research. It lasted until 2001 that the first 2-DE-maps with identified proteins of mouse adipose tissue were published [35, 36].

\section{Proteomics on mouse adipocytes}

Initial proteome studies were conducted on mouse 3T3-L1 cells to discriminate and identify differentially expressed proteins during adipocyte differentiation [37-39]. Many proteins were identified that had not been described before in 3T3-L1 cells but, dependent on the experimental conditions, each study revealed a different set of proteins next to commonly identified proteins. This demonstrated the power of proteomics studies but at the same time the sensitivity of the 3T3-L1 cellular proteome for different experimental conditions and the reproducibility limitation of the used 2-DE method. However, a commonly observed effect was the alteration of proteins involved in cytoskeletal re-arrangements, which is an important event during 3T3-L1 differentiation [40-42]. Furthermore, a proper functioning and remodelling of the cytoskeletal network is required for insulin signaling and GLUT4 translocation in 3T3-L1 adipocytes [43-47]. For instance, the actin-based motor protein myosin $5 a$ is a critical factor for anterograde movement of GLUT4 vesicles along the actin network in 3T3-L1 cells upon insulin-mediated stimulation [43].

Next to cytoskeletal arrangements, proteolytic events play a critical role in the initiation of preadipocyte differentiation [48-50]. One proteomics study revealed the protease inhibitor $\alpha-$ 2-macroglobulin to be involved in adipocyte differentiation [38]. Accumulation of intracellular $\alpha$-2-macroglobulin blocks differentiation while depletion of $\alpha$-2-macroglobulin by polyclonal antibodies again induced a spontaneous differentiation of 3T3-L1 cells. Similar results were found with freshly isolated mouse preadipocytes. Remarkably, the $\alpha$-2-macroglobulin protein found in this study was not mouse-derived. Apparently this protein was taken up from the 
culture medium that was supplemented with bovine serum. $\alpha$-2-macroglobulin, as protease inhibitor, might regulate this process by proteolysis suppression. Probably, preadipocytes take up this protein to inhibit spontaneous differentiation and upon inactivation of $\alpha-2$ macropglobulin differentiation is initiated.

Our group investigated proteome changes during 3T3-L1 differentiation followed by starvation [39]. We identified four categories of proteins: metabolic enzymes, growth regulatory proteins, proteins with functions in cytoskeleton re-arrangements and protein modifiers. Compared to 3T3-L1 differentiation we observed a non-reciprocal regulation of the glycolytic pathway when mature 3T3-L1adipocytes were subjected to caloric restriction. Several other proteins showed a similar behaviour. This indicates that although caloric restriction induces fat release, it results only in a limited preadipocyte protein expression pattern. Apparently, once differentiated, adipocytes do not completely return to their preadipocyte status upon caloric restriction. However, when 3T3-L1 adipocytes were subjected to caloric restriction in the presence with TNF- $\alpha$, the protein profile closely resembled the preadipocyte expression pattern. Stimulation of PPAR $\gamma$ inhibits TNF- $\alpha$ effects on mature 3T3-L1 cells [51]. Thus, we suggested that TNF- $\alpha$, in addition to caloric restriction, stimulates mature 3T3-L1 cells to return further to their preadipocyte status by a forced down-regulation of PPAR $\gamma$.

\section{Organelle proteomics}

Several proteomics studies have been conducted on specific cellular fractions isolated from 3T3-L1 cells, [52, 53], mice [54, 55] and humans [56]. Adipocytes store the triglycerides in lipid droplets but the protein composition of adipocyte lipid droplets remained elusive until identified by mass spectrometry [53]. Next to proteins also present on lipid droplets of other mammalian systems such as lipid metabolism enzymes and vesicular traffic controlling proteins, a number of adipocyte-specific ones were determined. Several of these were only identified from basal adipocytes such as 17- $\beta$-hydroxysteroid dehydrogenase type 7 and CGI-49, and others specifically under lipolytic conditions such as adipophilin, caveolin-1, tubulin and lipid-metabolizing enzymes. Presence of some of these proteins on lipid droplets was confirmed by immunofluorescence [53].

Recently, the nuclear proteome of 3T3-L1 cells was investigated during 5 consecutive days of differentiation using a quantitative five-plex SILAC LC-MSMS method [57]. With this novel approach differentially expressed proteins could be identified in a temporal fashion. In the nuclear fraction a total of 581 proteins were identified. Among these were e.g. the T-cell transcriptional regulator THO complex 4 that showed highest expression during mid-stage of differentiation and the chromatin modulator SNF2 $\alpha$ that was down-regulated during the start 


\section{CHAPTER 2}

of differentiation and remained at low expression level during the further differentiation. With this novel proteomics approach, this study contributed to a broader understanding of the transcriptional regulation of adipocyte differentiation.

With respect to other organelles, mitochondrial biogenesis and remodelling during 3T3-L1 differentiation was discovered as a novel property of adipocytes [52]. When the same experiment was repeated in the presence of the insulin sensitizing thiazolidinedione (TZD) drug rosiglitazone, again mitochondrial biosynthesis was observed. This was accompanied by increased expression of several mitochondrial proteins involved in fatty acid oxidation in the 3T3-L1 cells [52] as well as in the ob/ob mice [54]. The correlation between the expression levels of the identified proteins and their corresponding mRNA's was low, which implied a regulation of the mitochondrial proteins at the post-translational level.

\section{Effects of TZD's on the 3T3-L1 proteome}

The insulin sensitizers TZD's require adipose tissue for their therapeutic actions [58-60] although they stimulate fat cell differentiation, a known risk factor for insulin resistance. To investigate this paradoxical issue we examined the effect of two TZD's (pioglitazone and rosiglitazone) on the proteome of crude cell lysates of 3T3-L1 cells. We observed a pioglitazone- and rosiglitazone-mediated triglyceride accumulation during 3T3-L1 differentiation and induction of proteins involved in intracellular fatty acid transport, glycerol3-phosphate synthesis and gluconeogenesis from non-carbohydrate substrates. At the same time, both TZD's induced tricaboxylic acid cycle proteins, the complete fatty acid betaoxidation pathway and oxidative phosphorylation proteins. Thus, concomitant with increased triglyceride storage the TZD's also increased fatty acid catabolism [61]. In a parallel study the effect of rosiglitazone on mature 3T3-L1 adipocytes was investigated with a combined transcriptomics and proteomics study [62]. Decreased lipid contents were observed together with an mRNA and protein expression pattern that indicated a switch in metabolism towards lipid catabolism. Furthermore, rosiglitazone reduced adipokine expression both on mRNA and protein level, except for the insulin sensitizer adiponectin and apolipoprotein $E$ (ApoE). Together, these proteomics results added new information to support the view that TZD's exert their therapeutic effects through the fatty acid storage and catabolism capacity of the adipose tissue [63] and through a change in the adipokine profile.

\section{Proteomics on human adipocytes}

Despite its value for obesity research the 3T3-L1 model is hampered by its murine nature. A comparison of studies conducted on murine and human adipocytes increasingly showed 
differences between the two model systems regarding adipogenesis and adipokine secretion [64-67]. The value of mouse models to recapitulate human obesity is thus questionable. Detailed comparative proteome studies may reveal how well the mouse model is suited, at least on the proteome level, as a model for human obesity studies. Recently, an in-depth proteome analysis of 3T3-L1 adipocytes was published [68] using subcellular fractionation and high sensitive protein identification by sophisticated mass spectrometry. This strategy revealed 3,287 identified proteins and is currently the largest high confidence proteome map on adipocyte biology. A similar study with subcutaneous and visceral human adipocytes is still awaited but would allow a detailed comparison between the two species but also between the different human fat depots, which enables extensive functional characterization of the adipogenic process.

Proteome studies on human adipogenesis have been conducted with human adult stem cells derived from liposuction aspirates [69] and with mesenchymal stem cells [70, 71]. The human adult stem cells revealed a relative high percentage $(>40 \%)$ of identical proteins compared to mouse adipose tissue and 3T3-L1 cells. In addition, the functional properties of all identified proteins, e.g. cytoskeletal re-arrangements, metabolic and redox enzymes and protein processing, were highly similar compared to proteins earlier identified from murine models. These results might be explained by the applied 2-DE method that primarily reveals the high abundant proteins. (Subtle) differences that distinguish human adipocytes from murine adipocytes require in-depth investigation of the proteomes on a larger scale. Ideally this is done by subcellular fractionation of the adipose material and analysis with more sensitive methods like wide-range 2-DE and quantitative LC-MSMS.

Primary human adipocytes can be isolated from fresh adipose tissue but the propagation and manipulation possibilities of these cells are limited which make them less easy to use. Recently, adipose tissue explants have been used $[72,73]$ in proteome studies but these contain next to adipocytes also stromal vascular cells, which make it difficult to determine the origin of the identified proteins. Furthermore, the tissue explants themselves do not allow the examination of the adipocyte differentiation process.

Immortalized human preadipocytes appear a promising model system $[74,75]$ and developments in this field are ongoing. Other interesting cells are preadipocytes derived from a Simpson-Golabi-Behmel syndrome (SGBS) patient [76]. SGBS cells can be propagated for at least 30 generations without loosing their differentiation capacity. Differentiated SGBS cells are similar to human subcutaneous adipocytes with respect to morphology and biochemical characteristics but are easier to manipulate and therefore ideal to work with. Currently, the SGBS cells form an important in vitro model in the field of adipocyte biology research. 


\section{Analysis of adipokine secretion by proteomics}

Adipokines play an important role in normal physiology [77], however, disturbances in adipokine profiles coinciding the development of obesity are associated with the onset of metabolic complications [78-82]. Proteomics research has had a major impact on the identification of novel adipokines. Started with 3T3-L1 cells, different proteomic approaches have been applied to investigate the change in adipokine profiles during differentiation. Kratchmarova et al. [83] found 20 different secreted proteins by one-dimensional electrophoresis and LC-MSMS. Wang et al. [84] identified 41 different proteins by a combination of 2-DE and mass spectrometry. Together, both studies revealed several different proteins that had not been associated before with adipocyte secretion. More importantly, these studies showed for the first time the applicability of proteomics technology on another level of adipocyte biology, that is adipokine expression profiling.

\section{Adipocyte secretion routes}

Proteins bearing an N-terminal secretion signal peptide are secreted via the ER-Golgi pathway. However, next to this so-called classical pathway at least 3 additional routes of protein secretion are present in eukaryotic cells $[85,86]$. In a study on adipokine profiles from rat adipose cells 99 proteins out of 183 identified were considered as non-secreted because they lacked a secretion signal peptide [87]. However, structural analysis of proteins for signal secretion peptides might lead to an under-representation of adipocyte-derived proteins present in the extracellular space. Our group used a blocking strategy to discriminate between truly secreted proteins and proteins derived from cell leakage [84]. This method provided important information about the secretory properties of the identified proteins. For instance, cyclophilin A, which is known to be secreted from vascular smooth muscle cells [88], was identified as a truly adipocyte-secreted protein while the structure of this protein does not indicate secretory motives [84].

We showed that adipocyte secretion is stimulated by insulin but not on the transcriptional level of the secreted proteins. Instead, insulin promoted the transcription of post-translational processing proteins, particularly those that are involved in proteolysis [12]. The regulation of processing enzymes can increase protein secretion by shedding of transmembrane proteins. This process is another way to bring secretory proteins without secretion motives into the extracellular space $[89,90]$. For example, matrix metalloproteinases (MMP's) are involved in this phenomenon and several subtypes of these proteins have been identified as secreted proteins from adipocytes [12, 84, 87]. Whether these proteins are actively involved in proteolysis of adipocyte-bearing transmembrane proteins and as such stimulate adipokine secretion remains elusive. 
Another route for protein secretion by adipocytes is the release of microvesicles. A proteome analysis of microvesicles derived form 3T3-L1 cells revealed many proteins previously identified as adipokine [91]. Microvesicles can derive from direct budding from the plasma membrane [92] or from exocytosis of endosome-derived multivesicular bodies known as exosomes [93]. Both type of vehicles can be released in the intracellular space and are also found in body fluids. They function in the transfer of proteins and RNA molecules between cells. Furthermore, microvesicles appear to specifically target recipient cells (reviewed in [92, 93]). With respect to adipokines, this mechanism may function as a targeted communication pathway between the adipose tissue and other tissues like muscle, liver and intestine. Indepth proteome studies of adipocyte-derived microvesicles [94] will be necessary to further understand their functional properties, how they target their recipient cells and how they are related to obesity-related disorders.

\section{Human adipokines identified by proteomics}

In the human body the visceral and subcutaneous fat depots are important sources for adipokines present in the circulation. Especially the accumulation of visceral fat and the related increased free fatty acid flux together with a deregulation of visceral adipokine profiles are associated with insulin resistance, endothelial dysfunction and a proinflammatory state $[80,95]$. So far, several studies appeared on profiling of human adipokines, each applying a different experimental approach [71-73, 96, 97]. One study investigated mammary fat and its interstitial fluid by a combination of 2-DE, mass spectrometry and antibody array techniques [97]. 359 different proteins were identified, with functions in signal transduction, energy metabolism, cellular communication, cell growth and maintenance, and immune response. Although the relation between mammary fat and metabolic disturbances such as insulin resistance is less clear, obese woman are considered to be on a higher risk for developing post-menopausal breast cancer [98]. Mammary fatderived factors may be involved in this process as they are able to stimulate tumour cell growth.

So far, three studies appeared on adipokine profiles from freshly isolated human subcutaneous and visceral adipose tissue [72, 73, 96]. Secretomes of adipose tissue explants were examined by SDS-PAGE-LC-MSMS [72] and by antibody arrays [73], respectively. The visceral adipose tissue revealed 259 identified proteins from which 108 contained a secretion signal peptide. This left 151 proteins to be secreted via a non-classical pathway or even non-secreted. Indeed, the tissue explants appeared to contain some residual serum and intracellular proteins [72]. The secretome from subcutaneous fat was 


\section{CHAPTER 2}

investigated by an array containing antibodies against 120 different cytokines. Sixteen proteins were identified from which 5 were regulated by PPAR agonists [73].

Despite the high significance to investigate human adipokine expression, the above two studies could not discriminate the origin of their identified proteins. As adipose tissue explants mainly consist of adipocytes and stromal vascular cells it remains elusive from which cells the secreted proteins are derived. To resolve questions about adipose tissuederived proteins in the circulation this is a less severe problem. However, to dissect communication between adipocytes and other cell types this method is less useful. For instance, the question how macrophages are recruited into adipose tissue of obese persons is best studied by using pure cell cultures as secreted factors from differentiated adipocytes showed monocyte chemotactic activities [99].

Secretion profiles from undifferentiated and differentiated primary cultures of human adiposederived stem cells revealed 81 differentially expressed proteins as identified by a 2-DE approach [96]. About $72 \%$ of these proteins had already been discovered in previous proteomics studies showing a considerable overlap between rodent and human adipokine profiles. Since this study was conducted with 2-DE, the more subtle differences between the two species remain to be discovered. However, HSP47 (SERPINH1) a serpin family member, was identified as a new human adipocyte-secreted protein.

\section{Adipocyte extracellular matrix}

Extracellular matrix (ECM) proteins constitute a major part of the adipocyte secretion profile known today [12, 72, 83, 84, 96]. Remodelling of the ECM plays an important role in adipocyte differentiation $[100,101]$ and particularly MMP's have been demonstrated to be involved in this process in vitro $[102,103]$ and in vivo $[104,105]$. Previously, we determined a differential expression of several ECM proteins from mature 3T3-L1 cells by a novel stable isotope labelling method [106]. Based on these results it was concluded that ECM remodelling is also a functional property of mature 3T3-L1 adipocytes.

Recently it was shown that the ECM of human preadipocytes could be modulated by macrophage-secreted factors and that interstitial fibrosis in obese white adipose tissue coincides with infiltration of inflammatory cells $[107,108]$. This may imply that adipocyte-ECM modulation is associated with the inflammatory state found in the adipose tissue of obese subjects and may be linked to metabolic deregulation. A study by Khan et al. [109] demonstrated that the absence of the adipocyte ECM protein collagen $\mathrm{VI}$ resulted in an improvement of whole body energy homeostasis of ob/ob mice together with enlarged adipocyte size. This paradoxal finding was explained by a weakening of the ECM allowing increased triglyceride storage. As such, excess adipose tissue ECM formation during obesity 
may prevent storage of fat during excess energy intake and may induce plasma glucose and free fatty acid levels.

Together, the adipocyte ECM appears to play an important role in adipose tissue biology. However, the composition of the adipocyte ECM and how it is changed under different inflammatory and nutrient conditions is currently unclear.

\section{Proteomics on human adipose tissue biopsies}

So far, a limited number of proteome analyses have been conducted on human subcutaneous and visceral adipose tissue biopsies. These were from low and high-fat oxidizing obese subjects [110], polycystic ovary syndrome subjects [111] and non-diabetic obese subjects [112]. All three studies were performed with a 2-DE approach. Claessens et al [110] used a subtraction method to discriminate truly adipocyte proteins from contaminating blood-derived proteins and observed an induction of methylmalonatesemialdehyde dehydrogenase in low-fat oxidizing subjects. This may account for an increased valine catabolism as compensatory mechanism for reduced carbohydrate metabolism.

Upregulation of endoplasmic reticulum stress-related proteins was observed in fat biopsies from non-diabetic obese subjects compared to lean ones [112]. Although these proteins are involved in an unfolded protein response, most of these have also been assigned has generally stress-related proteins [24]. The same is true for the proteins identified by Corton et al. [111]. Together, these studies provided initial insight in proteome changes in adipose tissue from human subjects. In-depth proteome analysis may provide a further understanding of the biology of the adipose tissue with respect to obesity and obesity-related disorders. However, this requires complexity-reducing sample preparation and more sensitive analysis methods.

\section{Conclusion and future directions}

Since 2001 proteomics research on adipose biology has evolved and many proteins that had not been associated with adipocytes before have been identified. In addition, by means of proteomics data several novel biological features of adipocytes have been discovered. As indicated, none of the existing proteomics strategies can cover entire proteomes of mammalian systems at once. Thus the coverage of the total proteome of (pre)adipocytes and proteome changes during preadipocyte differentiation requires a combination of current analysis techniques. Subcellular fractionation, wide-scale 2-DE, high sensitive mass 


\section{CHAPTER 2}

spectrometry and high-density antibody arrays will all contribute to a further understanding of adipocyte biology.

Future applications for proteomics research in adipose biology are the further dissection of $i$ ) the molecular events during human adipogenesis, ii) the remodelling of the adipocyte ECM and iii) the link between obesity and obesity-related metabolic complications. Adipocytesecreted adipokines are supposed to play an important role in obesity-related disorders. However, how the change in adipokine profiles influence neighbour and remote target cells and how they induce metabolic complications remains elusive. A challenging task for proteomics researchers lies ahead.

\section{References}

1. Rosen, E.D. and B.M. Spiegelman, Adipocytes as regulators of energy balance and glucose homeostasis. Nature, 2006. 444(7121): p. 847-53.

2. Trayhurn, P., Endocrine and signalling role of adipose tissue: new perspectives on fat. Acta Physiol Scand, 2005. 184(4): p. 285-93.

3. Bluher, M., Adipose tissue dysfunction in obesity. Exp Clin Endocrinol Diabetes, 2009. 117(6): p. 241-50.

4. Goossens, G.H., The role of adipose tissue dysfunction in the pathogenesis of obesity-related insulin resistance. Physiol Behav, 2008. 94(2): p. 206-18.

5. Haslam, D., Obesity: a medical history. Obes Rev, 2007. 8 Suppl 1: p. 31-6.

6. Haslam, D., N. Sattar, and M. Lean, ABC of obesity. Obesity--time to wake up. Bmj, 2006. 333(7569): p. 640-2.

7. Haslam, D.W. and W.P. James, Obesity. Lancet, 2005. 366(9492): p. 1197-209.

8. Guan, H.P., et al., A futile metabolic cycle activated in adipocytes by antidiabetic agents. Nat Med, 2002. 8(10): p. 1122-8.

9. Burton, G.R., et al., Microarray analysis of gene expression during early adipocyte differentiation. Gene, 2002. 293(1-2): p. 21-31.

10. Soukas, A., et al., Distinct transcriptional profiles of adipogenesis in vivo and in vitro. J Biol Chem, 2001. 276(36): p. 34167-74.

11. Pradet-Balade, B., et al., Translation control: bridging the gap between genomics and proteomics? Trends Biochem Sci, 2001. 26(4): p. 225-9.

12. Wang, P., et al., Insulin modulates the secretion of proteins from mature 3T3-L1 adipocytes: a role for transcriptional regulation of processing. Diabetologia, 2006. 49(10): p. 2453-62.

13. Tyers, M. and M. Mann, From genomics to proteomics. Nature, 2003. 422(6928): p. 193-7. 
14. Sharan, R., I. Ulitsky, and R. Shamir, Network-based prediction of protein function. Mol Syst Biol, 2007. 3: p. 88.

15. Gorg, A., et al., 2-DE with IPGs. Electrophoresis, 2009. 30 Suppl 1: p. S122-32.

16. Rabilloud, T., Two-dimensional gel electrophoresis in proteomics: old, old fashioned, but it still climbs up the mountains. Proteomics, 2002. 2(1): p. 3-10.

17. Chen, G. and B.N. Pramanik, Application of LC/MS to proteomics studies: current status and future prospects. Drug Discov Today, 2009. 14(9-10): p. 465-71.

18. Domon, B. and R. Aebersold, Mass spectrometry and protein analysis. Science, 2006. 312(5771): p. 212-7.

19. Wingren, C. and C.A. Borrebaeck, Antibody-based microarrays. Methods Mol Biol, 2009. 509: p. 57-84.

20. Kingsmore, S.F., Multiplexed protein measurement: technologies and applications of protein and antibody arrays. Nat Rev Drug Discov, 2006. 5(4): p. 310-20.

21. Fichmann, J. and R. Westermeier, 2-D protein gel electrophoresis. An overview. Methods Mol Biol, 1999. 112: p. 1-7.

22. Marouga, R., S. David, and E. Hawkins, The development of the DIGE system: 2D fluorescence difference gel analysis technology. Anal Bioanal Chem, 2005. 382(3): p. 669-78.

23. Gade, D., et al., Evaluation of two-dimensional difference gel electrophoresis for protein profiling. Soluble proteins of the marine bacterium Pirellula sp. strain 1. J Mol Microbiol Biotechnol, 2003. 5(4): p. 240-51.

24. Wang, P., F.G. Bouwman, and E.C. Mariman, Generally detected proteins in comparative proteomics--a matter of cellular stress response? Proteomics, 2009. 9(11): p. 2955-66.

25. Heck, A.J. and J. Krijgsveld, Mass spectrometry-based quantitative proteomics. Expert Rev Proteomics, 2004. 1(3): p. 317-26.

26. Ong, S.E. and M. Mann, Mass spectrometry-based proteomics turns quantitative. Nat Chem Biol, 2005. 1(5): p. 252-62.

27. Yates, J.R., 3rd, Mass spectral analysis in proteomics. Annu Rev Biophys Biomol Struct, 2004. 33: p. 297-316.

28. Aebersold, R. and M. Mann, Mass spectrometry-based proteomics. Nature, 2003. 422(6928): p. 198-207.

29. Nilsson, P., et al., Towards a human proteome atlas: high-throughput generation of mono-specific antibodies for tissue profiling. Proteomics, 2005. 5(17): p. 4327-37.

30. Tegel, H., et al., High-throughput protein production--lessons from scaling up from 10 to 288 recombinant proteins per week. Biotechnol J, 2009. 4(1): p. 51-7. 
31. Ponten, F., K. Jirstrom, and M. Uhlen, The Human Protein Atlas--a tool for pathology. J Pathol, 2008. 216(4): p. 387-93.

32. Sidhu, R.S., Two-dimensional electrophoretic analyses of proteins synthesized during differentiation of 3T3-L1 preadipocytes. J Biol Chem, 1979. 254(21): p. 11111-8.

33. Student, A.K., R.Y. Hsu, and M.D. Lane, Induction of fatty acid synthetase synthesis in differentiating 3T3-L1 preadipocytes. J Biol Chem, 1980. 255(10): p. 4745-50.

34. Spiegelman, B.M. and H. Green, Control of specific protein biosynthesis during the adipose conversion of 3T3 cells. J Biol Chem, 1980. 255(18): p. 8811-18.

35. Lanne, B., et al., Thiourea enhances mapping of the proteome from murine white adipose tissue. Proteomics, 2001. 1(7): p. 819-28.

36. Sanchez, J.C., et al., The mouse SWISS-2D PAGE database: a tool for proteomics study of diabetes and obesity. Proteomics, 2001. 1(1): p. 136-63.

37. Welsh, G.I., et al., Proteome analysis of adipogenesis. Proteomics, 2004. 4(4): p. 1042-51.

38. Choi, K.L., et al., Proteomic analysis of adipocyte differentiation: Evidence that alpha2 macroglobulin is involved in the adipose conversion of 3T3 L1 preadipocytes. Proteomics, 2004. 4(6): p. 1840-8.

39. Renes, J., et al., Protein profiling of 3T3-L1 adipocyte differentiation and (tumor necrosis factor alpha-mediated) starvation. Cell Mol Life Sci, 2005. 62(4): p. 492-503.

40. Kawaguchi, N., et al., ADAM12 induces actin cytoskeleton and extracellular matrix reorganization during early adipocyte differentiation by regulating beta1 integrin function. J Cell Sci, 2003. 116(Pt 19): p. 3893-904.

41. Lieber, J.G. and R.M. Evans, Disruption of the vimentin intermediate filament system during adipose conversion of 3T3-L1 cells inhibits lipid droplet accumulation. J Cell Sci, 1996. 109 ( Pt 13): p. 3047-58.

42. Rodriguez Fernandez, J.L. and A. Ben-Ze'ev, Regulation of fibronectin, integrin and cytoskeleton expression in differentiating adipocytes: inhibition by extracellular matrix and polylysine. Differentiation, 1989. 42(2): p. 65-74.

43. Yoshizaki, T., et al., Myosin $5 a$ is an insulin-stimulated Akt2 (protein kinase Bbeta) substrate modulating GLUT4 vesicle translocation. Mol Cell Biol, 2007. 27(14): p. 5172-83.

44. Huang, J., et al., Disruption of microtubules ablates the specificity of insulin signaling to GLUT4 translocation in 3T3-L1 adipocytes. J Biol Chem, 2005. 280(51): p. 423006.

45. Olson, A.L., et al., Insulin promotes formation of polymerized microtubules by a phosphatidylinositol 3-kinase-independent, actin-dependent pathway in 3T3-L1 adipocytes. Endocrinology, 2003. 144(11): p. 5030-9. 
46. Kanzaki, M. and J.E. Pessin, Insulin-stimulated GLUT4 translocation in adipocytes is dependent upon cortical actin remodeling. J Biol Chem, 2001. 276(45): p. 42436-44.

47. Guilherme, A., et al., Perinuclear localization and insulin responsiveness of GLUT4 requires cytoskeletal integrity in 3T3-L1 adipocytes. J Biol Chem, 2000. 275(49): p. 38151-9.

48. Alexander, C.M., et al., Stromelysin-1 regulates adipogenesis during mammary gland involution. J Cell Biol, 2001. 152(4): p. 693-703.

49. Crandall, D.L., et al., Autocrine regulation of human preadipocyte migration by plasminogen activator inhibitor-1. J Clin Endocrinol Metab, 2000. 85(7): p. 2609-14.

50. Patel, Y.M. and M.D. Lane, Role of calpain in adipocyte differentiation. Proc Natl Acad Sci U S A, 1999. 96(4): p. 1279-84.

51. Souza, S.C., et al., BRL 49653 blocks the lipolytic actions of tumor necrosis factoralpha: a potential new insulin-sensitizing mechanism for thiazolidinediones. Diabetes, 1998. 47(4): p. 691-5.

52. Wilson-Fritch, L., et al., Mitochondrial biogenesis and remodeling during adipogenesis and in response to the insulin sensitizer rosiglitazone. Mol Cell Biol, 2003. 23(3): p. 1085-94.

53. Brasaemle, D.L., et al., Proteomic analysis of proteins associated with lipid droplets of basal and lipolytically stimulated 3T3-L1 adipocytes. J Biol Chem, 2004. 279(45): p. 46835-42.

54. Wilson-Fritch, L., et al., Mitochondrial remodeling in adipose tissue associated with obesity and treatment with rosiglitazone. J Clin Invest, 2004. 114(9): p. 1281-9.

55. Bluher, M., et al., Role of insulin action and cell size on protein expression patterns in adipocytes. J Biol Chem, 2004. 279(30): p. 31902-9.

56. Aboulaich, N., et al., Vectorial proteomics reveal targeting, phosphorylation and specific fragmentation of polymerase I and transcript release factor (PTRF) at the surface of caveolae in human adipocytes. Biochem J, 2004. 383(Pt 2): p. 237-48.

57. Molina, $\mathrm{H}$., et al., Temporal profiling of the adipocyte proteome during differentiation using a five-plex SILAC based strategy. J Proteome Res, 2009. 8(1): p. 48-58.

58. Hallakou, S., et al., Pioglitazone induces in vivo adipocyte differentiation in the obese Zucker fa/fa rat. Diabetes, 1997. 46(9): p. 1393-9.

59. de Souza, C.J., et al., Effects of pioglitazone on adipose tissue remodeling within the setting of obesity and insulin resistance. Diabetes, 2001. 50(8): p. 1863-71.

60. Oakes, N.D., et al., Thiazolidinediones increase plasma-adipose tissue FFA exchange capacity and enhance insulin-mediated control of systemic FFA availability. Diabetes, 2001. 50(5): p. 1158-65. 
61. Renes, J., et al., Thiazolidinediones regulate expression of proteins involved in triacylglyceride storage and fatty acid oxidation in 3T3-L1 (pre-)adipocytes. Adipocytes, 2006. 2(2): p. 75-91.

62. Wang, P., et al., Absence of an adipogenic effect of rosiglitazone on mature 3T3-L1 adipocytes: increase of lipid catabolism and reduction of adipokine expression. Diabetologia, 2007. 50(3): p. 654-65.

63. Yki-Jarvinen, H., Thiazolidinediones. N Engl J Med, 2004. 351(11): p. 1106-18.

64. Steppan, C.M., et al., The hormone resistin links obesity to diabetes. Nature, 2001. 409(6818): p. 307-12.

65. Smith, S.R., et al., Agouti expression in human adipose tissue: functional consequences and increased expression in type 2 diabetes. Diabetes, 2003. 52(12): p. 2914-22.

66. Heilbronn, L.K., et al., Relationship between serum resistin concentrations and insulin resistance in nonobese, obese, and obese diabetic subjects. J Clin Endocrinol Metab, 2004. 89(4): p. 1844-8.

67. Arner, P., Resistin: yet another adipokine tells us that men are not mice. Diabetologia, 2005. 48(11): p. 2203-5.

68. Adachi, J., et al., In-depth analysis of the adipocyte proteome by mass spectrometry and bioinformatics. Mol Cell Proteomics, 2007. 6(7): p. 1257-73.

69. DeLany, J.P., et al., Proteomic analysis of primary cultures of human adipose-derived stem cells: modulation by Adipogenesis. Mol Cell Proteomics, 2005. 4(6): p. 731-40.

70. Lee, H.K., et al., The proteomic analysis of an adipocyte differentiated from human mesenchymal stem cells using two-dimensional gel electrophoresis. Proteomics, 2006. 6(4): p. 1223-9.

71. Chiellini, C., et al., Characterization of human mesenchymal stem cell secretome at early steps of adipocyte and osteoblast differentiation. BMC Mol Biol, 2008. 9: p. 26.

72. Alvarez-Llamas, G., et al., Characterization of the human visceral adipose tissue secretome. Mol Cell Proteomics, 2007. 6(4): p. 589-600.

73. Klimcakova, E., et al., Profiling of adipokines secreted from human subcutaneous adipose tissue in response to PPAR agonists. Biochem Biophys Res Commun, 2007. 358(3): p. 897-902.

74. Rodriguez, A.M., et al., Adipocyte differentiation of multipotent cells established from human adipose tissue. Biochem Biophys Res Commun, 2004. 315(2): p. 255-63.

75. Darimont, C. and K. Mace, Immortalization of human preadipocytes. Biochimie, 2003. 85(12): p. 1231-3. 
76. Wabitsch, M., et al., Characterization of a human preadipocyte cell strain with high capacity for adipose differentiation. Int J Obes Relat Metab Disord, 2001. 25(1): p. 815.

77. Wang, P., et al., The secretory function of adipocytes in the physiology of white adipose tissue. J Cell Physiol, 2008. 216(1): p. 3-13.

78. Trayhurn, P., B. Wang, and I.S. Wood, Hypoxia in adipose tissue: a basis for the dysregulation of tissue function in obesity? Br J Nutr, 2008. 100(2): p. 227-35.

79. Trayhurn, P., C. Bing, and I.S. Wood, Adipose tissue and adipokines--energy regulation from the human perspective. J Nutr, 2006. 136(7 Suppl): p. 1935S-1939S.

80. Ritchie, S.A. and J.M. Connell, The link between abdominal obesity, metabolic syndrome and cardiovascular disease. Nutr Metab Cardiovasc Dis, 2007. 17(4): p. 319-26.

81. Trayhurn, P., Adipocyte biology. Obes Rev, 2007. 8 Suppl 1: p. 41-4.

82. Antuna-Puente, B., et al., Adipokines: the missing link between insulin resistance and obesity. Diabetes Metab, 2008. 34(1): p. 2-11.

83. Kratchmarova, I., et al., A proteomic approach for identification of secreted proteins during the differentiation of 3T3-L1 preadipocytes to adipocytes. Mol Cell Proteomics, 2002. 1(3): p. 213-22.

84. Wang, P., et al., Profiling of the secreted proteins during 3T3-L1 adipocyte differentiation leads to the identification of novel adipokines. Cell Mol Life Sci, 2004. 61(18): p. 2405-17.

85. Nickel, W. and C. Rabouille, Mechanisms of regulated unconventional protein secretion. Nat Rev Mol Cell Biol, 2009. 10(2): p. 148-55.

86. Nickel, W., The mystery of nonclassical protein secretion. A current view on cargo proteins and potential export routes. Eur J Biochem, 2003. 270(10): p. 2109-19.

87. Chen, X., et al., Quantitative proteomic analysis of the secretory proteins from rat adipose cells using a 2D liquid chromatography-MS/MS approach. J Proteome Res, 2005. 4(2): p. 570-7.

88. Jin, Z.G., et al., Cyclophilin A is a secreted growth factor induced by oxidative stress. Circ Res, 2000. 87(9): p. 789-96.

89. Huovila, A.P., et al., Shedding light on ADAM metalloproteinases. Trends Biochem Sci, 2005. 30(7): p. 413-22.

90. Mullberg, J., et al., The importance of shedding of membrane proteins for cytokine biology. Eur Cytokine Netw, 2000. 11(1): p. 27-38.

91. Aoki, N., et al., Identification and characterization of microvesicles secreted by 3T3L1 adipocytes: redox- and hormone-dependent induction of milk fat globule-epidermal growth factor 8-associated microvesicles. Endocrinology, 2007. 148(8): p. 3850-62. 
92. Cocucci, E., G. Racchetti, and J. Meldolesi, Shedding microvesicles: artefacts no more. Trends Cell Biol, 2009. 19(2): p. 43-51.

93. Simons, M. and G. Raposo, Exosomes - vesicular carriers for intercellular communication. Curr Opin Cell Biol, 2009.

94. Xiao, Z., et al., Proteomic analysis of extracellular matrix and vesicles. J Proteomics, 2009. 72(1): p. 34-45.

95. Rodriguez, A., et al., Visceral and subcutaneous adiposity: are both potential therapeutic targets for tackling the metabolic syndrome? Curr Pharm Des, 2007. 13(21): p. 2169-75.

96. Zvonic, S., et al., Secretome of primary cultures of human adipose-derived stem cells: modulation of serpins by adipogenesis. Mol Cell Proteomics, 2007. 6(1): p. 18-28.

97. Celis, J.E., et al., Identification of extracellular and intracellular signaling components of the mammary adipose tissue and its interstitial fluid in high risk breast cancer patients: toward dissecting the molecular circuitry of epithelial-adipocyte stromal cell interactions. Mol Cell Proteomics, 2005. 4(4): p. 492-522.

98. Harvie, M., L. Hooper, and A.H. Howell, Central obesity and breast cancer risk: a systematic review. Obes Rev, 2003. 4(3): p. 157-73.

99. Han, C.Y., et al., Adipocyte-derived serum amyloid $A 3$ and hyaluronan play a role in monocyte recruitment and adhesion. Diabetes, 2007. 56(9): p. 2260-73.

100. Kubo, Y., et al., Organization of extracellular matrix components during differentiation of adipocytes in long-term culture. In Vitro Cell Dev Biol Anim, 2000. 36(1): p. 38-44.

101. Antras, J., et al., Decreased biosynthesis of actin and cellular fibronectin during adipose conversion of 3T3-F442A cells. Reorganization of the cytoarchitecture and extracellular matrix fibronectin. Biol Cell, 1989. 66(3): p. 247-54.

102. Croissandeau, G., M. Chretien, and M. Mbikay, Involvement of matrix metalloproteinases in the adipose conversion of 3T3-L1 preadipocytes. Biochem J, 2002. 364(Pt 3): p. 739-46.

103. Bouloumie, A., et al., Adipocyte produces matrix metalloproteinases 2 and 9: involvement in adipose differentiation. Diabetes, 2001. 50(9): p. 2080-6.

104. Chun, T.H., et al., A pericellular collagenase directs the 3-dimensional development of white adipose tissue. Cell, 2006. 125(3): p. 577-91.

105. Chavey, C., et al., Matrix metalloproteinases are differentially expressed in adipose tissue during obesity and modulate adipocyte differentiation. J Biol Chem, 2003. 278(14): p. 11888-96.

106. Bouwman, F., J. Renes, and E. Mariman, A combination of protein profiling and isotopomer analysis using matrix-assisted laser desorption/ionization-time of flight 
mass spectrometry reveals an active metabolism of the extracellular matrix of 3T3-L1 adipocytes. Proteomics, 2004. 4(12): p. 3855-63.

107. Keophiphath, M., et al., Macrophage-secreted factors promote a profibrotic phenotype in human preadipocytes. Mol Endocrinol, 2009. 23(1): p. 11-24.

108. Henegar, C., et al., Adipose tissue transcriptomic signature highlights the pathological relevance of extracellular matrix in human obesity. Genome Biol, 2008. 9(1): p. R14.

109. Khan, T., et al., Metabolic dysregulation and adipose tissue fibrosis: role of collagen VI. Mol Cell Biol, 2009. 29(6): p. 1575-91.

110. Claessens, M., et al., Differential valine metabolism in adipose tissue of low and high fat-oxidizing obese subjects. Proteomics Clin. Appli., 2007. 1: p. 1306-1315.

111. Corton, M., et al., Proteomic analysis of human omental adipose tissue in the polycystic ovary syndrome using two-dimensional difference gel electrophoresis and mass spectrometry. Hum Reprod, 2008. 23(3): p. 651-61.

112. Boden, G., et al., Increase in endoplasmic reticulum stress-related proteins and genes in adipose tissue of obese, insulin-resistant individuals. Diabetes, 2008. 57(9): p. 2438-44. 


\section{Chapter 3}

\section{Identification of novel human adipocyte secreted proteins by using SGBS cells}

Anja Rosenow, Tabiwang N. Arrey, Freek G. Bouwman, Jean-Paul Noben, Martin Wabitsch, Edwin C.M. Mariman, Michael Karas, Johan Renes

Journal of Proteome Research. 2010; 9 5389-5401 


\title{
CHAPTER 3
}

\begin{abstract}
Adipose tissue is an endocrine organ secreting different types of proteins, known as adipokines. These adipokines play important roles in homeostasis and metabolism. Adipocyte differentiation leads to a change in adipokine secretion profile which is probably involved in disruption of homeostasis. Many adipokines have been identified but species differences and limitations of human adipose tissue material urged the need for better model systems. Here we used a human cell strain derived from a Simpson Golabi Behmel syndrome (SGBS) patient. SGBS cells have already been used in functional studies on adipocytes but not in a proteomic search for adipokines. In this study, 2-DE-MS/MS and nLCMALDI-MS/MS were applied to investigate secretion profiles of SGBS adipokines. A total of 80 secreted proteins were identified; 6 proteins are novel adipocyte secreted proteins, 20 proteins have not been detected before in human adipose material and 23 additional proteins previously detected in visceral adipose tissue have been found here secreted by SGBS-cells of subcutaneous origin. It can be concluded that SGBS cells are both a valid human cell model for adipocyte secretion profiling and for searching for novel human (pre)adipocytes secreted proteins.
\end{abstract}




\section{Introduction}

Obesity is a major health issue and is associated with development of several diseases and increased mortality. Consequently, obesity has a considerable impact on personal life quality as well as international economy [1-3]. Obesity is the result of an increase of body fat mass that leads to disruption of homeostasis. Body fat is stored in adipose tissue by an extension of existing adipocytes (hypertrophy) and by an increase of adipocyte number (hyperplasia) $[4,5]$.

Adipose tissue secretes different types of proteins which have autocrine, paracrine and endocrine effects on homeostasis and on metabolism. Among these secreted proteins are hormones, cytokines, neuron related proteins, extracellular matrix proteins as well as proteins involved in vascularization, lipid and glucose metabolism. Changes in their secretion during adipocyte differentiation leads to different circulation profiles, which are probably involved in initiating obesity-related disorders like insulin resistance, type 2 diabetes, cardiovascular diseases, cancer and other physiological disorders [1, 6-8].

Mouse 3T3-L1 cells have been a popular model to study the secretion and adipokine profile. However, limitations of using rodents as models are the species differences in adipokine expression, regulation and response. Well-known adipokines that show species differences are adiponectin, adipsin, leptin, and resistin [9-15]. This emphasizes the importance to characterize the adipocyte secretion profile, and to identify its dynamic changes during differentiation from a human source. Consequently, the number of studies that have used human cell material, in particular fat biopsies increased considerably over the last 5 years.

The use of human fat biopsies is limited with respect to the amount of preadipocytes and their differentiation capacity. To avoid these issues, human preadipocytes from subcutaneous adipose tissue of an infant with Simson-Golabi-Behmel syndrome (SGBS) can be used. These cells display a differentiation capacity of up to $90 \%$ and retain this capacity over at least 30 generations while being morphologically, biochemically and functionally similar to primary preadipocytes [16].

In the present study we have made use of those cells to investigate adipokine secretion in order to identify human-specific secretion profiles during preadipocyte differentiation. To discriminate between non-secreted, leaked proteins and actively secreted proteins that use the endoplasmatic reticulum (ER)/Golgi-dependent secretion pathway, the inhibitor Brefeldin A (BFA) was used $[17,18]$. To analyze changes in adipocyte secretion profile twodimensional (2-D) gel electrophoresis was applied followed by the identification of the secreted proteins by matrix-assisted laser desorption/ionization- time of flight/time of flight tandem mass spectrometry (MALDI TOF/TOF) and by liquid chromatography-electrospray ionization tandem mass spectrometry (LC-ESI MS/MS). Validation of the results was done by off-line LC followed by tandem mass spectrometry. 


\section{Methods}

\section{Materials}

Culture media, $0.5 \%$ trypsin-EDTA and 10,000 u/ml Penicillin together with 10,000 u/ml Streptomycin solutions were obtained from Invitrogen (Carlsbad, CA. USA). Fetal bovine serum (FBS) was from Bodinco (Alkmaar, The Netherlands). Additional cell culture supplements, Brefeldin A (BFA), protease inhibitor cocktail, 3-[(3-cholamidopropyl) dimethylamonio]-1-propanesulfonate (CHAPS), iodoacetamid, Ammonium bicarbonate, N, N, N',N'Tetramethylethylenediamine, a-cyano-4-hydroxyl-cinnamic acid (CHCA), trifluoroacetic acid (TFA) and acetonitrile (ACN) were purchased from Sigma-Aldrich (St. Louis, MO, USA). Ammonium persulfate (APS), protein assay kit, $20 \%$ sodium dodecyl sulfate (SDS) buffer, 10x Tris/glycine/SDS (TGS) buffer, 0.5 M Tris-HCL, pH 6.8, 1.5 M Tris-HCL, pH 8.8, urea and $30 \%$ acrylamide/bis solution were obtained from Bio-Rad Laboratories (Hercules, CA, USA). Immobilized pH gradient (IPG) buffer ( $\mathrm{pH} 3-11$, nonlinear), Dry-Strip cover fluid and immobiline Dry-Strip ( $\mathrm{pH} 3-11$, nonlinear, $24 \mathrm{~cm}$ ) were from GE Healthcare (Little Chalfont, UK).

\section{Cell culture}

Human Simpson-Golabi-Behmel syndrome (SGBS) cells were obtained from Prof. Dr. M. Wabitsch (University of Ulm, Germany) [16]. SGBS preadipocytes were seeded at a concentration of $8 \times 10^{5}-9 \times 10^{5}$ cells in $150 \mathrm{~mm}$ Petri dishes and cultured until 80 to $90 \%$ confluence in $10 \%$ FBS-containing DMEM/F12 medium supplemented with $66 \mathrm{nM}$ biotin, 33 nM D-pantothenic acid and 2\% penicillin/streptomycin antibiotics. Every 2 to 3 days the cells were washed with PBS buffer and medium was changed. The number of preadipocytes per Petri dish were $2.68 \pm 0.61 \times 10^{6}$ (mean $\left.\pm S D, n=6\right)$.

\section{Induction of differentiation}

To induce differentiation, confluent preadipocytes were washed twice with PBS buffer and the medium was changed to serum free DMEM/F12 medium containing $38 \mu \mathrm{g} / \mathrm{mL}$ human transferrin, $76 \mathrm{nM}$ insulin, $380 \mathrm{nM}$ cortisol and $0.76 \mathrm{nM}$ triiodothyronine, $1.9 \mathrm{mM}$ 1-methyl-3isobutyl-xanthine (IBMX), $95 \mathrm{nM}$ dexamethasone and $7.6 \mu \mathrm{M}$ rosiglitazone for the first 96 hours. Subsequently the cells were further differentiated in serum free DMEM/F12 medium containing $38 \mu \mathrm{g} / \mathrm{mL}$ human transferrin, $76 \mathrm{nM}$ insulin, $380 \mathrm{nM}$ cortisol and $0.76 \mathrm{nM}$ triiodothyronine. Every 2 to 3 days the medium was refreshed without washing the cells. After 12 days $65-85 \%$ of the preadipocytes were differentiated into mature adipocytes. The number of adipocytes per $150 \mathrm{~mm}$ Petri dish were $1.78 \pm 0.56 \times 10^{6}$ (mean $\pm \mathrm{SD}, \mathrm{n}=7$ ). 


\section{Protein sample preparation}

To discriminate between secreted proteins and cell-leakage proteins a similar BFA blocking strategy was used as previously described by Wang et al. [8]. Cells were treated with $20 \mu \mathrm{M}$ $\mathrm{BFA}$ at $37^{\circ} \mathrm{C}$ for $1 \mathrm{~h}$. Subsequently, the culture medium was removed, cells were washed twice with PBS and incubated with DMEM/F12 phenol red-free medium supplemented with $20 \mu \mathrm{M} \mathrm{BFA}$ at $37^{\circ} \mathrm{C}$ for $16 \mathrm{~h}$. Non-blocked cells were similarly treated without BFA but with an equal volume of DMSO as control. The media were collected, and supplemented with protease inhibitor cocktail. Afterwards, the media were centrifuged at $4^{\circ} \mathrm{C}, 230 \mathrm{xg}$ for 10 minutes. The supernatants were transferred to dialysis tubes (2-kDa molecular-weight cutoff, Carl Roth $\mathrm{GmbH}$, Karlsruhe, Germany) and dialyzed against $20 \mathrm{mM}$ ammonium bicarbonate at $4^{\circ} \mathrm{C}$ for $48 \mathrm{~h}$ with $8-10 \mathrm{x}$ buffer changes. Meanwhile, preadipocytes were trypsinized and counted with a haemocytometer and adipocytes were counted with a raster ocular. The dialyzed medium samples were freeze-dried, dissolved in fresh rehydration buffer (8 $\mathrm{M}$ urea, $2 \% \mathrm{w} / \mathrm{v}$ CHAPS and $65 \mathrm{mM}$ DTT) and centrifuged at $20000 \mathrm{xg}$ and $10^{\circ} \mathrm{C}$ for $30 \mathrm{~min}$. The protein concentrations were estimated by a Bradford-based protein assay kit (Bio-Rad) (12). All samples were stored at $-80^{\circ} \mathrm{C}$.

\section{Two-dimensional gel electrophoresis and MS/MS analysis}

\section{Two-dimensional gel electrophoresis}

Protein samples derived from $11 \times 10^{6}$ cells were used for 2-DE gel analysis according to Bouwman et al. [19] but with precast IPG strips (pH 3-11, $24 \mathrm{~cm}$, nonlinear). $75 \mu \mathrm{g}$ or $150 \mu \mathrm{g}$ (preparative gels) of total protein, diluted with the same lysis buffer used for sample preparation to a volume of $450 \mu \mathrm{l}$ and $0.5 \%$ (v/v) IPG, was loaded onto the IPG strips. For protein profiling, five independent replicates were made for each group of SGBS cells, i.e. SGBS adipocytes and SGBS preadipocytes with or without $20 \mu \mathrm{M}$ BFA. The gels were stained with flamingo protein stain according to the manufacturer's protocol (Bio-Rad Laboratories). To visualize the proteins, gels were scanned by the Molecular Imager FX (BioRad Laboratories).

\section{Image analysis}

Stained gels were processed by PDQuest 8.0 (Bio-Rad Laboratories). Data were normalized with respect to the total density of gel image. Each group was formed from samples of independent experiments with the same treatment. If the average spot intensity between the groups BFA treated and non-treated differed more than 2.0-fold and $p<0.05$ (obtained from Student's T-test PDQuest) this spot was regarded as significantly secreted. Similar criteria were used to indicate differentially expressed spots between preadipocytes and adipocytes. To refine the PDQuest 8.0 data further statistically evaluations were done. Therefore, spots 


\section{CHAPTER 3}

according to the PDQuest 8.0 Student's t-test that were regarded as significantly differentially secreted and expressed were re-evaluated by an Excel Student's t-test. The criteria for significant differences of the Excel Student's T-test were similar to the PDQuest Student's ttest criteria (spot intensity between the groups differed more than 2.0 -fold and $p<0.05$ ). The criteria for indicating a trend was a spot intensity differences of more than 2.0 -fold and $p<0.1$. For further investigations, gels with differentially expressed spots were re-stained with SYPRO Ruby Protein Stain (according manufacturer's protocol, Bio-Rad Laboratories).

\section{MS/MS and protein identification}

Excised spots were in-gel digested as described by Bouwman et al. [19]. To obtain peptide mass fingerprints $1 \mu \mathrm{l}$ of each peptide mixture and $1 \mu \mathrm{l}$ matrix solution $(10 \mathrm{mg} / \mathrm{ml} \mathrm{CHCA}$ in $50 \%$ ACN/0.1\% TFA) were spotted onto an Opti-TOF ${ }^{\mathrm{TM}} 384$ well plate (AB Sciex, Foster City, USA). The instrument was operated as described by Cranenburg et.al. [20]. Non-identified spots were further analyzed by LC-ESI MS/MS, as described by Dumont et al. [21].

\section{Database search}

To identify the proteins the peptide mass lists generated by MALDI-MS/MS were searched against the SwissProt database (SwissProt release 56.5; 402482 sequences) via Mascot (version 2.2.04; Matrix Science, London, U.K.) by the GPS explorer v3.9 software (AB Sciex, Foster City, USA). Taxonomy was set at all entries and for a more specific search on Homo sapiens. Only one missed cleavage was allowed, carbamidomethylation was set as a fixed modification and oxidation of methionine as a variable modification. The mass tolerance was $100 \mathrm{ppm}$ and there were no restrictions to protein mass and $\mathrm{pl}$. The protein identification criteria was a significant MASCOT probability score $(p<0.05)$.

With respect to LC-ESI-MS/MS, LCQ Xcalibur v2.0 SR2 raw files and spectra were selected from Proteome Discover1.0.0.43 Thermo Electron with following settings: minimal peak count 50 ; total intensity threshold 4000 ; and $S / N \geq 6$. Peal list were searched with Sequest v1.0.43 and Mascot v2.2.0.2 against EMBL-EBI International Protein Index database for human (version 3.66, 86379 entries). Search engine results were combined and validated by Scaffold v2.06.01 (Proteome Software) with minimum peptide and protein probability set to $\geq$ $95 \%$, followed by manual curation considering were mass spectral (annotation) quality.

\section{nLC-MALDI-MS/MS analysis}

\section{Digestion}

Each sample was concentrated using a vivaspin 500 centrifugal concentrator (3,000 MWCO PES Sartorius Stedim Biotech, Goettingen Germany). The lysis buffer was replaced with a $25 \mathrm{mM} \mathrm{NH}_{4} \mathrm{HCO}_{3}$ buffer ( $\mathrm{pH}$ 8.0). The disulfide bridges were reduced in $45 \mathrm{mM}$ DTT for $1 \mathrm{~h}$ 
at $60^{\circ} \mathrm{C}$ and $400 \mathrm{rpm}$ shaking in a Thermomixer comfort (Eppendorf, Hamburg Germany). The solution was allowed to cool to room temperature, and cysteines were alkylated in the presence of $100 \mathrm{mM}$ iodoacetamide for $45 \mathrm{~min}$ in the dark at room temperature. To avoid subsequent alkylation of trypsin, the reaction was quenched after 45 min by adding an additional $12.5 \mu \mathrm{l}$ of $45 \mathrm{mM}$ DTT and incubated for another $45 \mathrm{~min}$ at room temperature. Trypsin solution containing $10 \% \mathrm{ACN}$ was added to the reduced and alkylated samples to obtain a protein/enzyme ratio of $1: 50$ by weight and the solution was incubated overnight at $37^{\circ} \mathrm{C}$. Digestion was stopped by addition of $1 \mu \mathrm{l}$ of a $10 \%$ TFA solution.

\section{Nano-LC}

Peptide separation was performed on an EASY-nLC ${ }^{\mathrm{TM}}$ system (Proxeon Biosystems, Odense, Denmark) coupled to a SunCollect MALDI spotting device (Sunchrom, Friedrichsdorf, Germany). After vacuum concentration solvent A ( $8 \%$ ACN, $0.1 \%$ TFA) was added to increase the final volume to $15 \mu \mathrm{l}$. Prior to separation, the peptide was injected onto an in-house $\mathrm{C}_{18}$ packed $100 \mu \mathrm{m} \times 30 \mathrm{~mm}$ pre-column (Waters X-Bridge ${ }^{\mathrm{TM}} \mathrm{BEH} 180 \mathrm{C}_{18} 300$ A $3.5 \mu \mathrm{m}, 100 \mu \mathrm{m} \times 30 \mathrm{~mm}$ ) and desalted with $30 \mu \mathrm{l}$ solvent $A$ for $15 \mathrm{~min}$. The peptide mixture was separated on an in-house packed $100 \mu \mathrm{m} \times 150 \mathrm{~mm}$ column (Waters X-Bridge ${ }^{\mathrm{TM}}$ $\mathrm{BEH} 180 \mathrm{C}_{18} 300 \AA 3.5 \mu \mathrm{m}, 100 \mu \mathrm{m} \times 30 \mathrm{~mm}$ ) at a flow rate of $400 \mathrm{nl} / \mathrm{min}$. The gradient profile increased linear from $8-45 \%$ solvent $B(92 \%$ ACN, $0.1 \%$ TFA) in $100 \mathrm{~min}$, to $90 \%$ B in $20 \mathrm{~min}, 10 \mathrm{~min}$ at $90 \%, 5 \mathrm{~min}$ returned to $8 \% \mathrm{~B}$ and remained at $8 \%$ for another $5 \mathrm{~min}$.

The effluent from the LC was mixed via a tee (Upchurch Scientific, Washington, USA) with a matrix from an auxiliary pump (flow rate, $1.2 \mu \mathrm{l} / \mathrm{min}$ ) via a MicroTee and spotted every 20 seconds on a blank $123 \times 81 \mathrm{~mm}$ Opti-TOF $^{\text {TM }}$ LC/MALDI Insert metal target. This solution contained $3.5 \mathrm{mg} / \mathrm{ml} \mathrm{\alpha -CHCA} \mathrm{(Bruker} \mathrm{Daltonics,} \mathrm{Bremen,} \mathrm{Germany)} \mathrm{dissolved} \mathrm{in} 70 \% \mathrm{ACN}$, $30 \% \mathrm{H}_{2} \mathrm{O}, 0.1 \%$ TFA, spiked with $20 \mathrm{fmol}$ final amount of [Glu $\left.{ }^{1}\right]$-Fibrinopeptide $B$ (Bachem, Weil, Germany) per spot for internal calibration.

\section{MALDI MS/MS}

Mass spectra were acquired using a MALDI TOF/TOF ${ }^{T M} 4800$ Analyzer (AB Sciex, Darmstadt, Germany). All spectra were acquired in the positive reflector mode between 700 and $4500 \mathrm{~m} / \mathrm{z}$ with fixed laser intensity. A total of 750 laser shots per spot were accumulated. An 8-point plate model External calibration was performed using a Sequazyme ${ }^{\mathrm{TM}}$ Peptide Mass Standards Kit (AB Sciex, Darmstadt, Germany). The fragmentation was performed with collision energy of $1 \mathrm{kV}$ using air as collision gas at a pressure of $1 \times 10^{-6}$ Torr. To reduce sample consumption during measurement, stop conditions for MS/MS were defined: $A$ minimal number of 15 peaks above $45 \mathrm{~S} / \mathrm{N}$ with at least 12 accumulated sub-spectra, a minimum of 1250 and maximum of 2500 laser shots were recorded. To avoid unnecessary 


\section{CHAPTER 3}

multiple selections of identical precursor, MS/MS precursor selection was carried out via the instrument's software with job-wide interpretation. A total of 6 precursors per spot with a minimum signal-to-noise-ratio of 80 were selected for fragmentation. Potential matrix signals were removed from precursor selection by excluding all masses in the range from 700 to $1400 \mathrm{~m} / \mathrm{z}$ having values of $.030+/-.1 \mathrm{~m} / \mathrm{z}$, as well as from the internal calibrant.

\section{Database searches and interpretation}

Mascot Generic Format (MGF) files were retrieved from each MALDI MS/MS spectrum using the built-in Peaks2Mascot feature, exporting up to 65 peaks per MS/MS spectrum, each requiring a minimum signal-to-noise of 5 . The MGF files were processed using the Mascot ${ }^{\mathrm{TM}}$ database search engine v2.2.03 (Matrix Science Ltd., London, UK). The following settings were used: taxonomy: homo sapiens; enzyme: trypsin; allowed miss cleavages for trypsin: 3; fixed modification: carboxymethylation of cysteine; variable modification: oxidation of methionin: MS precursor mass tolerance: $50 \mathrm{pmm}$; MS/MS mass tolerance: $0.5 \mathrm{Da}$. The search was performed against the SwissProt database containing 466739 entries as of November $30^{\text {th }}$ 2009. A decoy database consisting of same-length random protein sequences is automatically generated and searched. False discovery rate is calculated by dividing the absolute number of decoy hits through the sum of decoy and true hits against the actual database.

All statistical analyses were based on peptides having Mascot ${ }^{\mathrm{TM}} \mathrm{MS} / \mathrm{MS}$ ion scores exceeding the "identity or extensive homology threshold" $(p<0.05)$. In the case of multiple fragmentations of identical precursors, due to recurrence in repetitive runs, only data from the highest scoring peptide were kept.

Cell culture supplement-based protein contaminations like insulin and albumin were identified but not further listed.

\section{Analysis of secreted protein candidates}

For verification of secreted protein candidates a SwissProt analysis of amino acid sequences with SignalP and SecretomeP was performed. SignalP 3.0 predicts the presence and location of signal peptide cleavage sites in amino acid sequences. SecretomeP 2.0 predicts non-classical, in particular not signal peptide-triggered protein secretion. In this process the non-classically secreted proteins should obtain an NN-score above the threshold of 0.5 but not at the same time be predicted to contain a signal peptide. 


\section{Results}

\section{Characterization of the secretome of SGBS cells by the 2-DE-method}

Approximately $75 \%$ of all SGBS cells were differentiated over a period of 12 days into mature adipocytes. Successful adipogenesis was characterized by visible fat droplets (Figure 1). For analyzing the secretome cell culture medium with and without BFA from $90 \%$ confluent preadipocytes (adipogenesis day 0 ) and from mature adipocytes (adipogenesis day 12) was harvested, dialyzed and freeze dried. $75 \mu \mathrm{g}$ of total secretion protein was separated on 2-DE gels and after visualization quantitative analyses were performed by PDQuest. 4 representative 2-DE gels with clear distinctive patterns of secreted proteins from preadipocytes and adipocytes as well as their master gel created by PDQuest are shown in Figure 2.
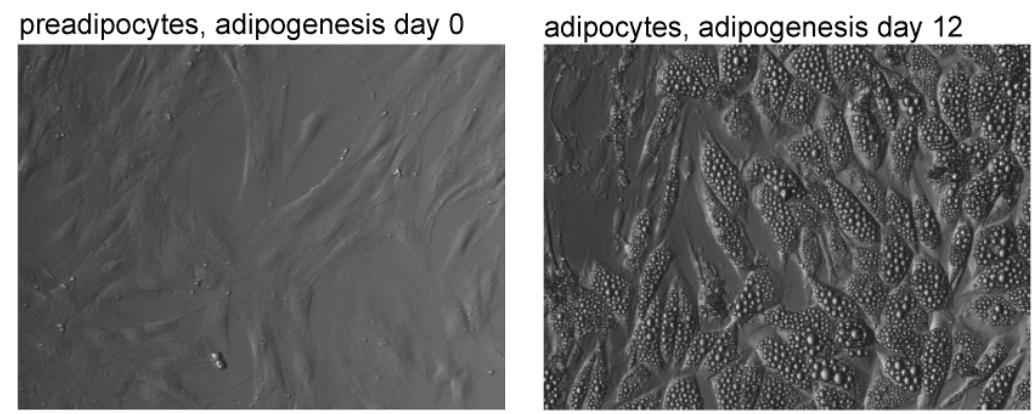

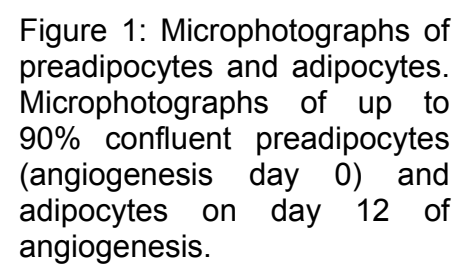
angiogenesis

From a total of 1243 spots 130 spots were selected by PDQuest as secreted and cell type specific spots, which were subjected to further analyses by mass spectrometry (Supplement 1). A total of 59 spots were identified by MALDI MS/MS or LC-ESI MS/MS. Within this group of 59 spots 55 were significantly secreted and 4 further spots showed a trend for secretion. These 59 spots are indicated on the master gel in Figure 2B and listed in Table 1. They include 35 different proteins of which 10 proteins are secreted with different isoforms. From all 59 spots 39 were significantly differentially expressed between preadipocytes and adipocytes and 4 spots were differentially expressed by trend. Based on this data 36 spots were highly expressed by adipocytes and 7 spots highly expressed by preadipocytes. 
A
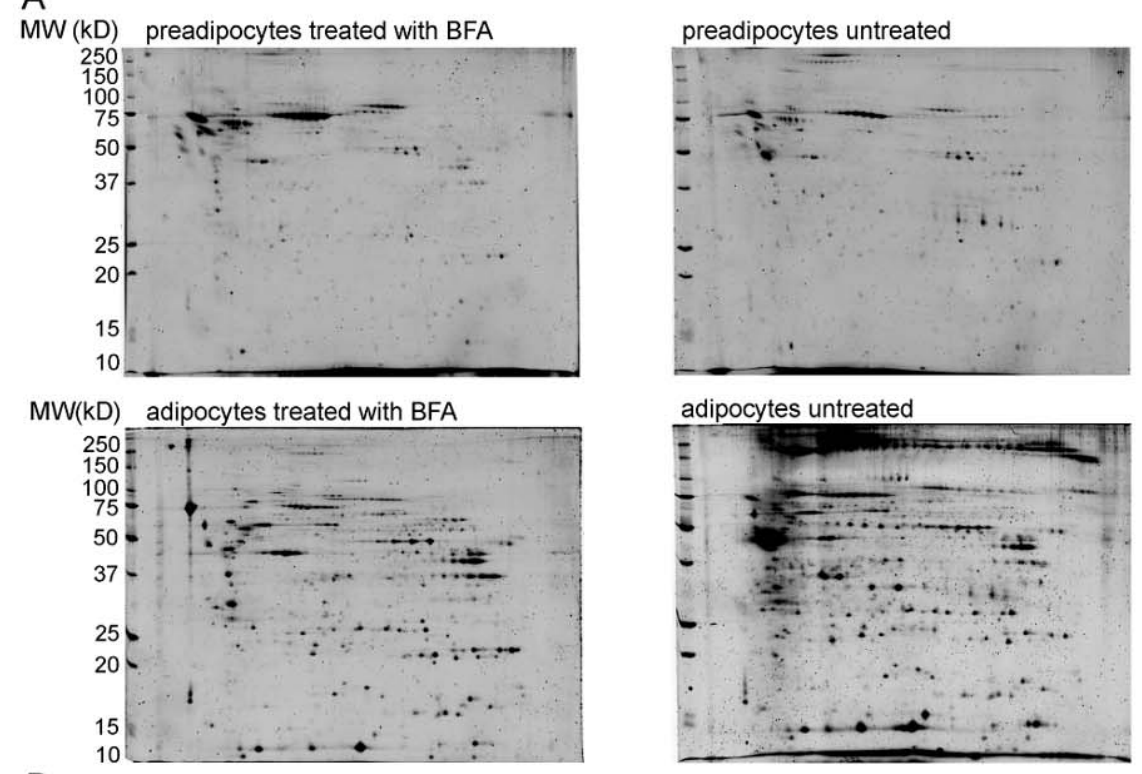

B

$\mathrm{MW}(\mathrm{kD})$

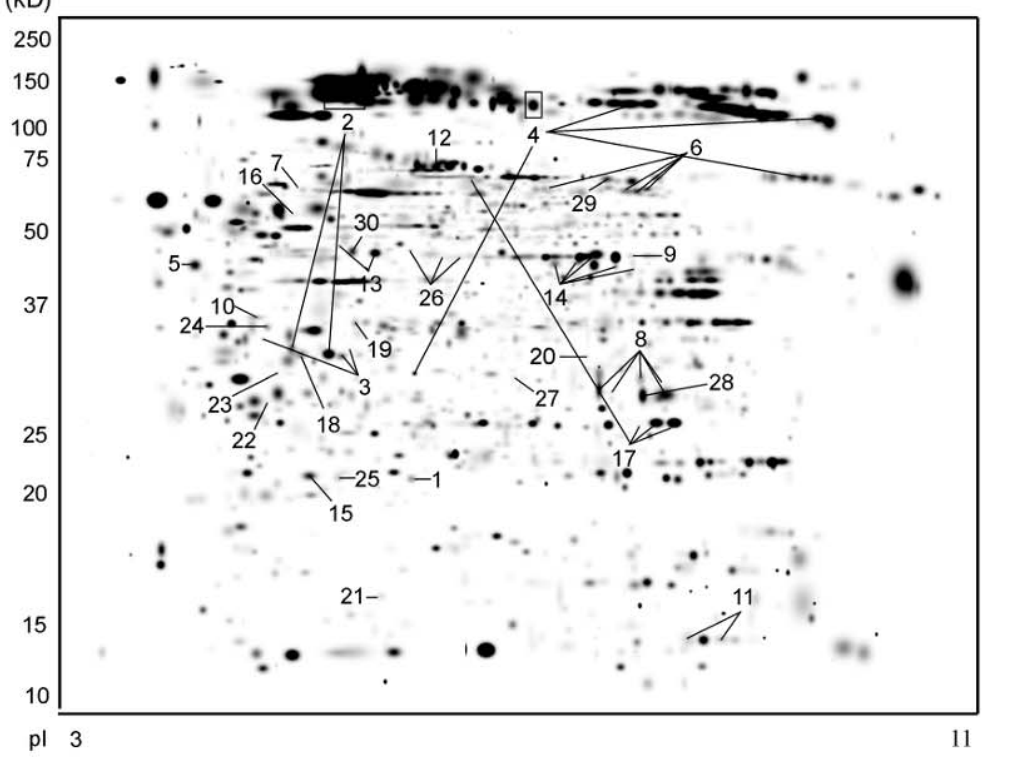

Figure 2: 2-DE gel electrophoresis gels of SGBS cells. 2-DE gels of medium-derived secreted proteins during differentiation of SGBS cells. A. Representative gels of medium-derived secreted protein of up to $90 \%$ confluent preadipocytes and 12 day differentiated adipocytes in presence or absence of $20 \mu \mathrm{M}$ BFA. B.Master gel with identified spots. Spot numbers refer to ID numbers of Tables 1 and 2.

The identified proteins were categorized in 3 groups: secreted, known as non-secreted and mixed spots. The proteins annotated as secreted were sub-categorized into extracellular matrix (HSPG, collagens, SPARC etc...), turnover (MMP-2, TIMP-1 and PCPE-1), regulation/signaling (Apo-E, PEDF, PAl-1 etc...), immune regulation (Complement factor $\mathrm{D}$ ), and others (transgelin-2) (see Table 1). SignalP analysis was used to validate BFA blocking experiments and it classified all proteins as classical secreted proteins. Vitamin D-binding protein (spot 16) is known as a classical secreted protein. Because it is identified from a mixed spot no validation by the BFA blocking experiment and no further determination of its 
expression level could be made. The category "known as non-secreted proteins" contains 4 proteins (annexin A5, GAPDH, LDH-A, and cyclophilin A) that are quantified as secreted proteins. SignalP-analysis identified no signal peptide cleavage sites but for annexin A5 and LDH-A secretomeP-analysis identified a NN score higher than 0.5 (0.503 and 0.614$)$ which is an indicator of non-classical secretion proteins. All 4 proteins showed increased expression in adipocytes. The "mixed spot" category contains 11 spots with a total of 21 different proteins. Based on the BFA blocking experiments 9 of these spots were annotated as significant secreted spots and 7 of them showed prominent expression in adipocytes.

Table 1: Identification of secreted proteins of SGBS cells by 2-DE-MS/MS

\begin{tabular}{|c|c|c|c|c|c|}
\hline ID & $\begin{array}{l}\text { Access- } \\
\text { ion } \\
\text { number }\end{array}$ & Protein name & $\begin{array}{c}\mathrm{MW} \\
{[\mathrm{kDa}]}\end{array}$ & Cell type & $\begin{array}{l}\text { Cell } \\
\text { type } p- \\
\text { value }\end{array}$ \\
\hline \multicolumn{6}{|c|}{ secreted } \\
\hline 1 & P98160 & $\begin{array}{l}\text { Basement membrane-specific heparan sulfate proteoglycan } \\
\text { core protein (HSPG) (perlecan) }\end{array}$ & 23 & adipocyte & 1.0E-02 \\
\hline \multirow[t]{6}{*}{2} & P02452 & Collagen alpha- $1(\mathrm{I})$ chain & 36 & both & 0.5 \\
\hline & & & 35 & both & 0.6 \\
\hline & & & 211 & both & 0.1 \\
\hline & & & 216 & adipocyte & 5.3E-08 \\
\hline & & & 215 & both & 0.2 \\
\hline & & & 220 & adipocyte & $1.5 \mathrm{E}-02$ \\
\hline \multirow[t]{3}{*}{3} & P02461 & Collagen alpha-1(III) chain & 37 & adipocyte & 1.4E-03 \\
\hline & & & 36 & adipocyte & 0.1 \\
\hline & & & 35 & adipocyte & 4.9E-09 \\
\hline \multirow[t]{5}{*}{4} & P08123 & Collagen alpha-2(I) chain & 33 & both & 0.4 \\
\hline & & & 194 & adipocytes & 1.6E-02 \\
\hline & & & 87 & adipocyte & 2.1E-03 \\
\hline & & & 165 & adipocyte & 4.4E-03 \\
\hline & & & 191 & adipocyte & 7.7E-03 \\
\hline 5 & P09486 & SPARC (Osteonectin) & 48 & both & 0.3 \\
\hline \multirow[t]{5}{*}{6} & Q15582 & $\begin{array}{l}\text { Transforming growth factor-beta-induced protein ig-h3 (Beta } \\
\text { ig-h3) }\end{array}$ & 78 & preadipocyte & 8.4E-07 \\
\hline & & & 77 & preadipocyte & 2.7E-02 \\
\hline & & & 76 & preadipocyte & 1.9E-02 \\
\hline & & & 75 & preadipocyte & 4.9E-02 \\
\hline & & turnover & & & \\
\hline 7 & P08253 & $\begin{array}{l}72 \text { kDa type IV collagenase (Matrix metalloproteinase-2) } \\
\text { (MMP-2) }\end{array}$ & 78 & both & 0.3 \\
\hline \multirow[t]{5}{*}{8} & P01033 & $\begin{array}{l}\text { Metalloproteinase inhibitor } 1 \text { (Tissue inhibitor of } \\
\text { metalloproteinases) (TIMP-1) }\end{array}$ & 30 & preadipocyte & 7.3E-03 \\
\hline & & & 31 & preadipocyte & 7.5E-02 \\
\hline & & & 31 & preadipocyte & 3.7E-02 \\
\hline & & & 32 & both & 0.3 \\
\hline & & & 33 & adipocytes & 0.1 \\
\hline \multirow[t]{2}{*}{9} & Q15113 & Procollagen C-endopeptidase enhancer 1 (PCPE-1) & 51 & adipocyte & $3.9 \mathrm{E}-03$ \\
\hline & & regulation/signaling & & & \\
\hline 10 & P02649 & Apolipoprotein E (Apo-E) & $\begin{array}{l}\text { mixed } \\
\text { spot }\end{array}$ & mixed spot & $\begin{array}{l}\text { mixed } \\
\text { spot }\end{array}$ \\
\hline \multirow[t]{2}{*}{11} & P01034 & Cystatin-C (Neuroendocrine basic polypeptide) & 10 & both & 0.6 \\
\hline & & & 10 & adipocyte & 4.4E-07 \\
\hline 12 & P06396 & Gelsolin isoform 1 (Actin-depolymerizing factor) & 98 & adipocyte & 4.1E-03 \\
\hline \multirow[t]{2}{*}{13} & P36955 & $\begin{array}{l}\text { Pigment epithelium-derived factor (PEDF) ( Serpin-F1) } \\
\text { (EPC-1) }\end{array}$ & 54 & adipocyte & $1.2 \mathrm{E}-10$ \\
\hline & & & 52 & adipocyte & 6.6E-10 \\
\hline
\end{tabular}


Table 1: Identification of secreted proteins of SGBS cells by 2-DE-MS/MS

\begin{tabular}{|c|c|c|c|c|c|}
\hline ID & $\begin{array}{l}\text { Access- } \\
\text { ion } \\
\text { number }\end{array}$ & Protein name & $\begin{array}{l}\mathrm{MW} \\
{[\mathrm{kDa}]}\end{array}$ & Cell type & $\begin{array}{l}\text { Cell } \\
\text { type } p- \\
\text { value }\end{array}$ \\
\hline & & $\begin{array}{l}\text { secreted } \\
\text { regulation/signaling }\end{array}$ & & & \\
\hline \multirow[t]{5}{*}{14} & P05121 & Plasminogen activator inhibitor 1 (PAI-1) & 49 & both & 0.5 \\
\hline & & & 48 & both & 0.6 \\
\hline & & & 49 & both & 0.3 \\
\hline & & & 48 & both & 0.2 \\
\hline & & & 51 & adipocytes & 8.6E-02 \\
\hline 15 & $\mathrm{P} 02753$ & Retinol-binding protein 4 (PRBP) (RBP) & 22 & adipocyte & $9.6 \mathrm{E}-10$ \\
\hline \multirow[t]{2}{*}{16} & P02774 & Vitamin D-binding protein (DBP) (VDB) & $\begin{array}{l}\text { mixed } \\
\text { spot }\end{array}$ & mixed spot & $\begin{array}{l}\text { mixed } \\
\text { spot }\end{array}$ \\
\hline & & immune regulation & & & \\
\hline \multirow[t]{5}{*}{17} & P00746 & $\begin{array}{l}\text { Complement factor D (C3 convertase activator) (Properdin } \\
\text { factor D) ( Adipsin) }\end{array}$ & 85 & adipocyte & $1.8 \mathrm{E}-04$ \\
\hline & & & 28 & adipocyte & 2.1E-09 \\
\hline & & & 27 & adipocyte & $1.2 \mathrm{E}-09$ \\
\hline & & & 28 & adipocyte & $6.6 \mathrm{E}-08$ \\
\hline & & not known as secreted proteins & & & \\
\hline 18 & P08758 & Annexin A5 & 35 & adipocyte & 1.6E-07 \\
\hline 19 & P04406 & Glyceraldehyde-3-phosphate dehydrogenase (GAPDH) & 36 & adipocyte & $5.9 \mathrm{E}-10$ \\
\hline 20 & P00338 & L-lactate dehydrogenase A chain (LDH-A) & 35 & adipocyte & $3.5 \mathrm{E}-02$ \\
\hline \multirow[t]{2}{*}{21} & P62937 & $\begin{array}{l}\text { Peptidyl-prolyl cis-trans isomerase A (PPlase A) (Cyclophilin } \\
\text { A) }\end{array}$ & 12 & both & $2.5 \mathrm{E}-03$ \\
\hline & & mixed spot & & & \\
\hline \multirow[t]{4}{*}{22} & P22676 & Calretinin (CR) (29 kDa calbindin) & 30 & adipocyte & 7.1E-06 \\
\hline & P31946 & $14-3-3$ protein beta/alpha & & & \\
\hline & P61981 & $14-3-3$ protein gamma & & & \\
\hline & P63104 & 14-3-3 protein zeta/delta & & & \\
\hline \multirow[t]{2}{*}{10} & P02649 & Apolipoprotein E (Apo-E) & 40 & adipocyte & 7.1E-11 \\
\hline & P07951 & Tropomyosin beta chain (Tropomyosin-2) & & & \\
\hline \multirow[t]{5}{*}{16} & P11021 & 78 kDa glucose-regulated protein (HSPA5) & 66 & both & 0.9 \\
\hline & P02774 & Vitamin D-binding protein (DBP) (VDB) & & & \\
\hline & P31150 & $\begin{array}{l}\text { Rab GDP dissociation inhibitor alpha (Rab GDI alpha) } \\
\text { (Guanosine diphosphate dissociation inhibitor 1) }\end{array}$ & & & \\
\hline & Q02818 & Nucleobindin-1 (CALNUC) & & & \\
\hline & P10809 & $\begin{array}{l}60 \mathrm{kDa} \text { heat shock protein (Heat shock protein } 60) \text { (HSP-60) } \\
\text { (Mitochondrial matrix protein P1) }\end{array}$ & & & \\
\hline \multirow[t]{2}{*}{23} & P08758 & Annexin A5 & 34 & adipocyte & 5.3E-08 \\
\hline & P02461 & Collagen alpha-1(III) chain & & & \\
\hline \multirow[t]{2}{*}{24} & P02649 & Apolipoprotein E (Apo-E) & 39 & adipocyte & 4.7E-08 \\
\hline & P29692 & Elongation factor 1 -delta isoform (1EF-1-delta) & & & \\
\hline \multirow[t]{2}{*}{25} & P98160 & $\begin{array}{l}\text { Basement membrane-specific heparan sulfate proteoglycan } \\
\text { core protein (HSPG) (perlecan) }\end{array}$ & 23 & adipocyte & $1.8 \mathrm{E}-11$ \\
\hline & P32119 & Peroxiredoxin-2 (Thioredoxin peroxidase 1) & & & \\
\hline \multirow[t]{3}{*}{26} & P36955 & $\begin{array}{l}\text { Pigment epithelium-derived factor (PEDF) ( Serpin-F1) } \\
\text { (EPC-1) }\end{array}$ & 53 & adipocyte & 1.1E-03 \\
\hline & P06733 & Alpha-enolase ( Enolase 1) & 51 & adipocyte & 2.8E-08 \\
\hline & & & 50 & adipocyte & 2.7E-09 \\
\hline \multirow[t]{2}{*}{27} & P08123 & Collagen alpha-2(I) chain & 32 & both & 0.2 \\
\hline & P01033 & $\begin{array}{l}\text { Metalloproteinase inhibitor } 1 \text { (Tissue inhibitor of } \\
\text { metalloproteinases) (TIMP-1) }\end{array}$ & & & \\
\hline \multirow[t]{3}{*}{28} & P01033 & $\begin{array}{l}\text { Metalloproteinase inhibitor } 1 \text { (Tissue inhibitor of } \\
\text { metalloproteinases) (TIMP-1) }\end{array}$ & 30 & both & 0.1 \\
\hline & P18669 & $\begin{array}{l}\text { Phosphoglycerate mutase } 1 \text { (Phosphoglycerate mutase } \\
\text { isozyme B) (PGAM-B) }\end{array}$ & & & \\
\hline & O14818 & Proteasome subunit alpha type-7 & & & \\
\hline \multirow[t]{2}{*}{29} & Q15582 & $\begin{array}{l}\text { Transforming growth factor-beta-induced protein ig-h3 (Beta } \\
\text { ig-h3) }\end{array}$ & 86 & both & 0.3 \\
\hline & P05121 & Plasminogen activator inhibitor 1 (PAl-1) & & & \\
\hline
\end{tabular}


Table 1: Identification of secreted proteins of SGBS cells by 2-DE-MS/MS

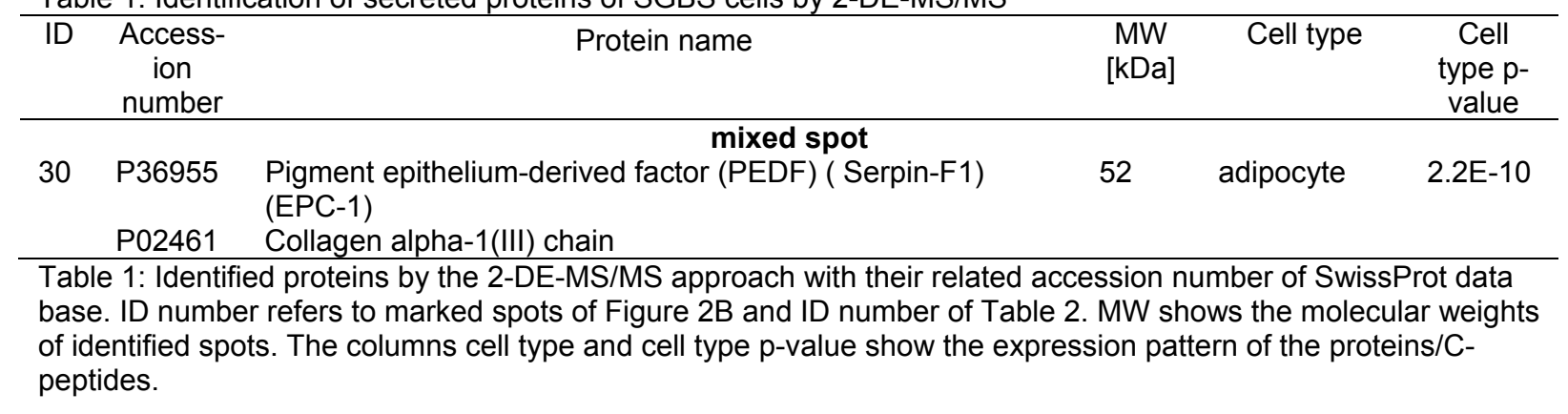

\section{nLC-MALDI-MS/MS approach for a more detailed characterization of the SGBS secretome}

To validate the 2-DE analysis and to obtain additional secretion proteins of the SBGS cells a $\mathrm{nLC}-M A L D I-M S / M S$ analysis of medium samples from preadipocytes and adipocytes was performed. A total of 75 and 182 proteins of preadipocytes and adipocytes, respectively, were identified (Supplement 2). Based on SignalP and/or SecretomeP analysis, 78 of the identified proteins can be described as secreted proteins. Of these proteins, 8 were only detected in preadipocytes, 35 were only detected in adipocytes and 35 proteins were detected in both cell types (Table 2).

Table 2: Identification of secreted proteins of SGBS cells by nLC-MALDI-MS/MS

\begin{tabular}{|c|c|c|c|c|}
\hline ID & $\begin{array}{c}\text { Access- } \\
\text { ion } \\
\text { number }\end{array}$ & Protein name & $\begin{array}{c}\text { Preadipocyte } \\
\text { peptides }\end{array}$ & $\begin{array}{c}\text { Adipocyte } \\
\text { peptides }\end{array}$ \\
\hline \multicolumn{5}{|c|}{$\begin{array}{c}\text { secreted } \\
\text { extracellular matrix }\end{array}$} \\
\hline & P07355 & Annexin A2 & not found & 7 \\
\hline 1 & P98160 & $\begin{array}{l}\text { Basement membrane-specific heparan sulfate proteoglycan } \\
\text { core protein (HSPG) (perlecan) }\end{array}$ & 12 & 2 \\
\hline \multirow[t]{2}{*}{2} & P02452 & Collagen alpha- $1(\mathrm{I})$ chain & 70 & 34,20 \\
\hline & $\mathrm{P} 02458$ & Collagen alpha-1(II) chain & 11 & 2,1 \\
\hline \multirow[t]{5}{*}{3} & $\mathrm{P} 02461$ & Collagen alpha-1(III) chain & 27 & 20,23 \\
\hline & P02462 & Collagen alpha-1(IV) chain & not found & 2,3 \\
\hline & P20908 & Collagen alpha- $1(\mathrm{~V})$ chain & 12 & 3,1 \\
\hline & P12109 & Collagen alpha- 1 (VI) chain & 19 & 19,11 \\
\hline & Q99715 & Collagen alpha- 1 (XII) chain & 9 & 3 \\
\hline \multirow[t]{18}{*}{4} & P08123 & Collagen alpha-2(I) chain & 85 & 34,31 \\
\hline & P08572 & Collagen alpha-2(IV) chain & 17 & 8,6 \\
\hline & P05997 & Collagen alpha-2(V) chain & 12 & 4,3 \\
\hline & $\mathrm{P} 12110$ & Collagen alpha-2(VI) chain & not found & 6,1 \\
\hline & P25940 & Collagen alpha-3(V) chain & not found & 3 \\
\hline & $\mathrm{P} 12111$ & Collagen alpha-3(VI) chain & not found & 7,1 \\
\hline & P29279 & $\begin{array}{l}\text { Connective tissue growth factor ( Hypertrophic chondrocyte- } \\
\text { specific protein } 24 \text { ) }\end{array}$ & not found & 1 \\
\hline & P07585 & Decorin (Bone proteoglycan II) (PG-S2) (PG40) & not found & 2,1 \\
\hline & Q07507 & Dermatopontin (tyrosine-rich acidic matrix protein) (tramp) & not found & $\overrightarrow{1}$ \\
\hline & Q12805 & $\begin{array}{l}\text { EGF-containing fibulin-like extracellular matrix protein } 1 \\
\text { (Fibulin-3) }\end{array}$ & 4 & 3 \\
\hline & P35555 & Fibrillin-1 (FBN1) & 11 & 4 \\
\hline & $\mathrm{P} 02751$ & Fibronectin (FN) (Cold-insoluble globulin) & 98 & 45,26 \\
\hline & P23142 & Fibulin-1 (FBLN1) & 4 & 3 \\
\hline & P09382 & Galectin-1 (Lectin galactoside-binding soluble 1) & not found & 4,3 \\
\hline & Q08380 & $\begin{array}{l}\text { Galectin-3-binding protein ( Lectin galactoside-binding } \\
\text { soluble 3-binding protein) }\end{array}$ & 6 & 4,2 \\
\hline & P07942 & Laminin subunit beta-1 (laminin B1 chain) & not found & 1 \\
\hline & P11047 & Laminin subunit gamma-1 (laminin B2 chain) & not found & 1 \\
\hline & P51884 & Lumican ( Keratan sulfate proteoglycan lumican) & 4 & 2 \\
\hline
\end{tabular}


Table 2: Identification of secreted proteins of SGBS cells by nLC-MALDI-MS/MS

\begin{tabular}{|c|c|c|c|c|}
\hline ID & $\begin{array}{c}\text { Access- } \\
\text { ion } \\
\text { number }\end{array}$ & Protein name & $\begin{array}{c}\text { Preadipocyte } \\
\text { peptides }\end{array}$ & $\begin{array}{l}\text { Adipocyte } \\
\text { peptides }\end{array}$ \\
\hline & & $\begin{array}{c}\text { secreted } \\
\text { extracellular matrix }\end{array}$ & & \\
\hline & P14543 & Nidogen-1 (entactin) & not found & 9,3 \\
\hline & Q15063 & Periostin (PN) (Osteoblast-specific factor 2) & 7 & 2 \\
\hline \multirow[t]{3}{*}{5} & P09486 & SPARC (Osteonectin) & 11 & 10,9 \\
\hline & P07996 & Thrombospondin-1 & 32 & 12,2 \\
\hline & P35442 & Thrombospondin-2 & 5 & not found \\
\hline \multirow[t]{2}{*}{6} & Q15582 & $\begin{array}{l}\text { Transforming growth factor-beta-induced protein ig-h3 (Beta } \\
\text { ig-h3) }\end{array}$ & 32 & 3 \\
\hline & & turnover & & \\
\hline \multirow[t]{2}{*}{7} & P08253 & $\begin{array}{l}72 \text { kDa type IV collagenase (Matrix metalloproteinase-2) } \\
\text { (MMP-2) }\end{array}$ & 16 & 9,2 \\
\hline & Q9Y4K0 & Lysyl oxidase homolog 2 (Lysyl oxidase-like protein 2) & 10 & not found \\
\hline \multirow[t]{2}{*}{8} & P01033 & $\begin{array}{l}\text { Metalloproteinase inhibitor } 1 \text { (Tissue inhibitor of } \\
\text { metalloproteinases) (TIMP-1) }\end{array}$ & 12 & 4,3 \\
\hline & P16035 & $\begin{array}{l}\text { Metalloproteinase inhibitor } 2 \text { (Tissue inhibitor of } \\
\text { metalloproteinases 2) (TIMP-2) }\end{array}$ & not found & 1,1 \\
\hline \multirow[t]{6}{*}{9} & Q15113 & Procollagen C-endopeptidase enhancer 1 (PCPE-1) & 4 & 5 \\
\hline & P28300 & Protein-lysine 6-oxidase (Lysyl oxidase) & 6 & not found \\
\hline & & regulation/signaling & & \\
\hline & P07108 & $\begin{array}{l}\text { Acyl-CoA-binding protein (ACBP) (Diazepam-binding } \\
\text { inhibitor) }\end{array}$ & not found & 3,3 \\
\hline & Q15848 & Adiponectin & not found & 3,1 \\
\hline & P02765 & $\begin{array}{l}\text { Alpha-2-HS-glycoprotein (Ba-alpha-2-glycoprotein) (Alpha-2- } \\
\text { Z-globulin) (Fetuin-A) }\end{array}$ & 1 & not found \\
\hline \multirow[t]{3}{*}{10} & P02649 & Apolipoprotein E (Apo-E) & not found & 9 \\
\hline & P27797 & Calreticulin (CRP55) (calregulin) & not found & 2,1 \\
\hline & O43852 & Calumenin (Crocalbin) & 4 & 5,1 \\
\hline \multirow[t]{2}{*}{11} & P01034 & Cystatin-C (Neuroendocrine basic polypeptide) & not found & 5 \\
\hline & Q12841 & Follistatin-related protein 1 (Follistatin-like 1) & 3 & 5,4 \\
\hline \multirow[t]{10}{*}{12} & P06396 & Gelsolin (Actin-depolymerizing factor) & not found & 7 \\
\hline & P07093 & $\begin{array}{l}\text { Glia-derived nexin (GDN) (Protease nexin I) (Protease } \\
\text { inhibitor } 7 \text { ) }\end{array}$ & 5 & 1 \\
\hline & P17936 & Insulin-like growth factor-binding protein 3 (IBP-3) & 6 & 1,1 \\
\hline & P22692 & Insulin-like growth factor-binding protein 4 (IBP-4) & 5 & 3,3 \\
\hline & P24593 & Insulin-like growth factor-binding protein 5 (IBP-5) & not found & 2,2 \\
\hline & P24592 & Insulin-like growth factor-binding protein 6 (IBP-6) & 3 & 1 \\
\hline & Q16270 & Insulin-like growth factor-binding protein 7 (IBP-7) & 6 & not found \\
\hline & P05452 & Tetranectin (TN) ( Plasminogen kringle 4-binding protein) & not found & 1 \\
\hline & Q14767 & $\begin{array}{l}\text { Latent-transforming growth factor beta-binding protein } 2 \\
\text { (LTBP-2) }\end{array}$ & 4 & not found \\
\hline & Q92626 & Peroxidasin homolog (Vascular peroxidase 1) & 1 & 1 \\
\hline 13 & P36955 & $\begin{array}{l}\text { Pigment epithelium-derived factor (PEDF)( Serpin-F1) (EPC- } \\
\text { 1) }\end{array}$ & not found & 7,4 \\
\hline \multirow[t]{9}{*}{14} & P05121 & Plasminogen activator inhibitor 1 (PAl-1) & 21 & 4 \\
\hline & P41222 & Prostaglandin-H2 D-isomerase (PGDS2) & not found & 2 \\
\hline & 000391 & Sulfhydryl oxidase 1 (hQSOX) & 14 & 1 \\
\hline & P78539 & Sushi repeat-containing protein SRPX & not found & 1 \\
\hline & & immune regulation & & \\
\hline & P10909 & Clusterin (Complement cytolysis inhibitor)(CLI) & not found & 1 \\
\hline & P00736 & Complement $\mathrm{C} 1 \mathrm{r}$ subcomponent & not found & 7 \\
\hline & P09871 & Complement C1s subcomponent & not found & 3 \\
\hline & P01024 & Complement C3 & not found & 7 \\
\hline \multirow[t]{4}{*}{17} & P00746 & $\begin{array}{l}\text { Complement factor D (C3 convertase activator) (Properdin } \\
\text { factor D) ( Adipsin) }\end{array}$ & not found & 4,3 \\
\hline & 014498 & $\begin{array}{l}\text { Immunoglobulin superfamily containing leucine-rich repeat } \\
\text { protein }\end{array}$ & 3 & not found \\
\hline & Q9H293 & Interleukin-25 (IL-25) & 1 & not found \\
\hline & P14174 & Macrophage migration inhibitory factor (MIF) & 1 & 1,1 \\
\hline
\end{tabular}


Table 2: Identification of secreted proteins of SGBS cells by nLC-MALDI-MS/MS

\begin{tabular}{|c|c|c|c|c|}
\hline ID & $\begin{array}{c}\text { Access- } \\
\text { ion } \\
\text { number }\end{array}$ & Protein name & $\begin{array}{l}\text { Preadipocyte } \\
\text { peptides }\end{array}$ & $\begin{array}{l}\text { Adipocyte } \\
\text { peptides }\end{array}$ \\
\hline \multicolumn{5}{|c|}{ secreted } \\
\hline & P26022 & $\begin{array}{l}\text { Pentraxin-related protein PTX3 (Tumor necrosis factor- } \\
\text { inducible gene } 14 \text { protein) }\end{array}$ & 15 & 3 \\
\hline & P05155 & Plasma protease C1 inhibitor (C1 Inh) & not found & 3,2 \\
\hline \multicolumn{5}{|c|}{ other } \\
\hline & Q2M329 & Coiled-coil domain-containing protein 96 & not found & 1 \\
\hline & P37802 & Transgelin-2 (SM22-alpha homolog) & not found & 1 \\
\hline \multicolumn{5}{|c|}{ not known as secreted } \\
\hline 18 & P08758 & Annexin A5 & not found & 2 \\
\hline 19 & P04406 & Glyceraldehyde-3-phosphate dehydrogenase (GAPDH) & not found & 1 \\
\hline 20 & P00338 & L-lactate dehydrogenase A chain (LDH-A) & 2 & 5,1 \\
\hline 21 & P62937 & $\begin{array}{l}\text { Peptidyl-prolyl cis-trans isomerase A (PPlase A) (Cyclophilin } \\
\text { A) }\end{array}$ & not found & 2,1 \\
\hline
\end{tabular}

The secreted proteins were sub-categorized into extracellular matrix (HSPG, collagens, FN, SPARC etc...), turnover (MMP-2, TIMP-1, TIMP-2 and PCPE-1), regulation/signaling (adiponectin, Apo-E, PEDF, PAI-1, etc...), immune regulation (Complement factor D, MIF etc...), and others (coiled-coil domain-containing protein 96, transgelin-2). Based on SecretomeP analysis, acyl-CoA-binding protein, coiled-coil domain-containing protein 96, and transgelin-2 can be reported as non-classical secretion proteins with $\mathrm{NN}$ scores of 0.758 , 0.622 and 0.784 , respectively.

All 2-DE-identified proteins in the categories "secreted" and "known as non-secreted spot" except retinol-binding protein 4 (spot 15) and vitamin D-binding protein (spot 16) were also identified by nLC-MALDI-MS/MS (Table 2). As such, 90\% of the $212-\mathrm{DE}-\mathrm{MS} / \mathrm{MS}$ identified proteins were also identified by nLC-MALDI-MS/MS. Furthermore, 58 additional secreted proteins were identified. On the other hand, the 2-DE analysis revealed only $24 \%$ of the 78 secreted proteins that were identified by nLC-MALDI-MS/MS (Figure 3). In total, by using both approaches, 80 secreted proteins from SGBS preadipocytes and adipocytes were identified. 


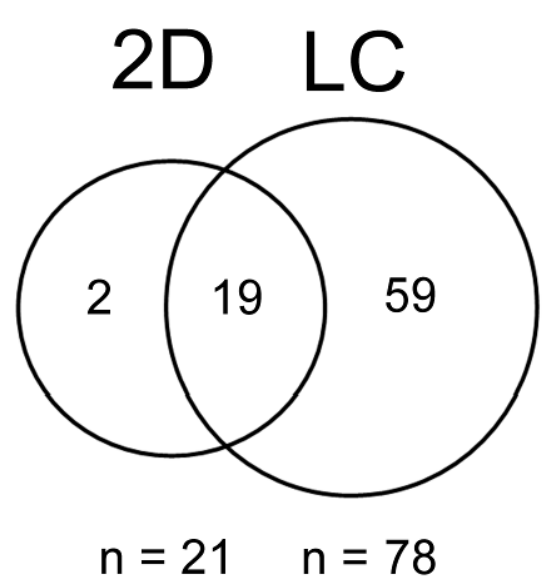

Figure 3: Distribution of secreted protein identification of SGBS cells. Venn diagram of the distribution of all 80 identified secreted protein by both proteomics approaches. $\mathrm{n}$ represents the identified secreted proteins of each method.

\section{Validation of the identified secretome}

The total data set of the identified secretome was compared to adipocyte proteomics literature describing the currently known secretome from human visceral [22, 23] and subcutaneous [24-26] as well as rodent [8, 27-31] adipose tissue (Table 3). 8 of the 80 secreted proteins (lysyl oxidase homolog 2, alpha-2-HS-glycoprotein, glia-derived nexin, insulin-like growth factor-binding protein (IBP) 3 , and IBP-5, sushi repeat-containing protein, immunoglobulin superfamily containing leucine-rich repeat protein, and coiled-coil domaincontaining protein 96) have not been reported in this literature. Further literature investigations confirm that 6 of these 8 proteins (lysyl oxidase homolog 2, alpha-2-HSglycoprotein, glia-derived nexin, sushi repeat-containing protein, immunoglobulin superfamily containing leucine-rich repeat protein, and coiled-coil domain-containing protein 96) have not been reported as adipocyte secreted protein before and therefore they can be described as novel (pre)adipocytes secreted proteins. In contrast, IBP-3 is already known as a secreted protein from human subcutaneous adipocytes and 3T3-L1 cells [32, 33]. IBP-5 mRNA levels are shown in human subcutaneous adipose tissue but is only described as an adipocyte secreted protein from a pig adipose tissue [34-36]. All of those proteins were identified by the nLC-MALDI-MS/MS approach. In addition, 13 proteins (some collagens, FBN1, IL-25, MIF, etc...) have been reported for rodents, but not for human adipocytes. Vitamin D-binding protein is the only one of these 13 proteins that was identified by the 2-DE-MS/MS approach. A total of 60 identified secreted proteins were described in human adipocyte studies. 36 of those were described in human visceral as well as in subcutaneous adipocyte/adipose tissue studies. 2 proteins were described only in human subcutaneous studies, and 23 were described only in human visceral adipose tissue studies. Since SGBS cells are of subcutaneous origin, our study adds 43 ( 6 novel +14 mammal specific +23 visceral specific) proteins to the secretome of human subcutaneous (pre)adipocytes. 
Table 3: Comparison of identified secreted proteins with previous proteomic adipocyte studies

\begin{tabular}{|c|c|c|c|}
\hline \multicolumn{4}{|c|}{ Knoun in literaturo } \\
\hline & human visceral & $\begin{array}{l}\text { human } \\
\text { subcutaneous }\end{array}$ & rodent \\
\hline \multicolumn{4}{|c|}{ extracellular matrix } \\
\hline Annexin A2 & [23] & not found & {$[27][30]$} \\
\hline $\begin{array}{l}\text { Basement membrane-specific heparan sulfate } \\
\text { proteoglycan core protein (HSPG) (perlecan) }\end{array}$ & {$[22][23]$} & [24] & {$[27][30]$} \\
\hline Collagen alpha-1(I) chain & {$[22][23]$} & {$[24][26]$} & {$[8][27][31]$} \\
\hline Collagen alpha-1(II) chain \# & not found & not found & [30] \\
\hline Collagen alpha-1(III) chain & {$[22][23]$} & [24] & {$[8][30]$} \\
\hline Collagen alpha-1(IV) chain \# & not found & not found & [8] \\
\hline Collagen alpha-1(V) chain & [23] & not found & {$[8][31]$} \\
\hline Collagen alpha-1(VI) chain & [23] & {$[24]$} & [8][27][29][30][31] \\
\hline Collagen alpha-1(XII) chain & [22] & not found & {$[30]$} \\
\hline Collagen alpha-2(I) chain & [22][23] & [24] & {$[8][27][30]$} \\
\hline Collagen alpha-2(IV) chain & [22] & not found & {$[8][31]$} \\
\hline Collagen alpha-2(V) chain \# & not found & not found & [30] \\
\hline Collagen alpha-2(VI) chain & {$[22][23]$} & [24] & {$[8][27][29][31]$} \\
\hline Collagen alpha-3(V) chain \# & not found & not found & [30] \\
\hline Collagen alpha-3(VI) chain & {$[22][23]$} & [24] & {$[27][30]$} \\
\hline $\begin{array}{l}\text { Connective tissue growth factor ( Hypertrophic } \\
\text { chondrocyte-specific protein 24) \# }\end{array}$ & not found & not found & [29] \\
\hline Decorin (Bone proteoglycan II) (PG-S2) (PG40) & {$[23]$} & [24] & {$[30]$} \\
\hline $\begin{array}{l}\text { Dermatopontin (tyrosine-rich acidic matrix protein) } \\
\text { (tramp) }\end{array}$ & [22] & [26] & not found \\
\hline $\begin{array}{l}\text { EGF-containing fibulin-like extracellular matrix protein } \\
1 \text { (Fibulin-3) }\end{array}$ & {$[22][23]$} & not found & [30] \\
\hline Fibrillin-1 (FBN1) \# & not found & not found & [30] \\
\hline Fibronectin (FN) (Cold-insoluble globulin) & {$[22][23]$} & [24] & {$[27][30][31]$} \\
\hline Fibulin-1 (FBLN1) & [22] & not found & [30] \\
\hline Galectin-1 (Lectin galactoside-binding soluble 1) \# & not found & not found & [8][27] \\
\hline $\begin{array}{l}\text { Galectin-3-binding protein ( Lectin galactoside-binding } \\
\text { soluble 3-binding protein) }\end{array}$ & {$[22][23]$} & [24] & [30] \\
\hline Laminin subunit beta-1 (laminin B1 chain) & {$[22][23]$} & not found & [30] \\
\hline Laminin subunit gamma-1 (laminin B2 chain) & {$[22][23]$} & [24] & [30] \\
\hline Lumican ( Keratan sulfate proteoglycan lumican) & {$[22][23]$} & [24] & {$[30][31]$} \\
\hline Nidogen-1 (entactin) & [22][23] & [24] & {$[27][30][31]$} \\
\hline Periostin (PN) (Osteoblast-specific factor 2) & {$[22][23]$} & [24] & [31] \\
\hline SPARC (Osteonectin) & {$[22][23]$} & [24][26] & {$[8][28][29][30][31]$} \\
\hline Thrombospondin-1 & {$[22]$} & not found & not found \\
\hline Thrombospondin-2 & {$[22]$} & not found & [30] \\
\hline $\begin{array}{l}\text { Transforming growth factor-beta-induced protein ig-h3 } \\
\text { (Beta ig-h3) }\end{array}$ & {$[22][23]$} & [24] & {$[30]$} \\
\hline \multicolumn{4}{|c|}{ turnover } \\
\hline $\begin{array}{l}72 \text { kDa type IV collagenase (Matrix metalloproteinase- } \\
\text { 2) (MMP-2) }\end{array}$ & {$[22][23]$} & not found & [31] \\
\hline Lysyl oxidase homolog 2 (Lysyl oxidase-like protein 2) & not found & not found & not found \\
\hline $\begin{array}{l}\text { Metalloproteinase inhibitor } 1 \text { (Tissue inhibitor of } \\
\text { metalloproteinases) (TIMP-1) }\end{array}$ & {$[22][23]$} & {$[24][25]$} & {$[28][30]$} \\
\hline $\begin{array}{l}\text { Metalloproteinase inhibitor } 2 \text { (Tissue inhibitor of } \\
\text { metalloproteinases 2) (TIMP-2) }\end{array}$ & {$[23]$} & [25] & [8][31] \\
\hline Procollagen C-endopeptidase enhancer 1 (PCPE-1) & [22][23] & [24] & {$[8][27][31]$} \\
\hline Protein-lysine 6-oxidase (Lysyl oxidase) \# & not found & not found & [8] \\
\hline \multicolumn{4}{|c|}{ regulation/signaling } \\
\hline $\begin{array}{l}\text { Acyl-CoA-binding protein (ACBP) (Diazepam-binding } \\
\text { inhibitor) }\end{array}$ & [23] & not found & not found \\
\hline Adiponectin & {$[22][23]$} & {$[25]$} & {$[8][27][28][30][31]$} \\
\hline $\begin{array}{l}\text { Alpha-2-HS-glycoprotein (Ba-alpha-2-glycoprotein) } \\
\text { (Alpha-2-Z-globulin) (Fetuin-A) * }\end{array}$ & not found & not found & not found \\
\hline Apolipoprotein E (Apo-E) & {$[23]$} & not found & {$[30]$} \\
\hline Calreticulin (CRP55) (calregulin) & [22][23] & {$[24][26]$} & [31] \\
\hline
\end{tabular}


Table 3: Comparison of identified secreted proteins with previous proteomic adipocyte studies

\begin{tabular}{|c|c|c|c|}
\hline \multirow[t]{2}{*}{ Protein name } & \multicolumn{3}{|c|}{ Known in literature } \\
\hline & human visceral & $\begin{array}{l}\text { human } \\
\text { subcutaneous }\end{array}$ & rodent \\
\hline \multicolumn{4}{|c|}{ regulation/signaling } \\
\hline Calreticulin (CRP55) (calregulin) & {$[22][23]$} & {$[24][26]$} & [31] \\
\hline Calumenin (Crocalbin) & not found & {$[23][25]$} & [28][30] \\
\hline Cystatin-C (Neuroendocrine basic polypeptide) & {$[22][23]$} & not found & [28][29] \\
\hline Follistatin-related protein 1 (Follistatin-like 1) & [23] & {$[24][26]$} & {$[30]$} \\
\hline Gelsolin isoform 1 (Actin-depolymerizing factor) & {$[22][23]$} & not found & {$[8][28][29][30]$} \\
\hline $\begin{array}{l}\text { Glia-derived nexin (GDN) (Protease nexin I) (Protease } \\
\text { inhibitor 7) * }\end{array}$ & not found & not found & not found \\
\hline Insulin-like growth factor-binding protein 3 (IBP-3) ** & not found & not found & not found \\
\hline Insulin-like growth factor-binding protein 4 (IBP-4) & [23] & not found & [30] \\
\hline Insulin-like growth factor-binding protein 5 (IBP-5) ** & not found & not found & not found \\
\hline Insulin-like growth factor-binding protein 6 (IBP-6) & [23] & not found & not found \\
\hline Insulin-like growth factor-binding protein 7 (IBP-7) & {$[22][23]$} & [23] & not found \\
\hline $\begin{array}{l}\text { Tetranectin (TN) ( Plasminogen kringle 4-binding } \\
\text { protein) \# }\end{array}$ & not found & not found & {$[30]$} \\
\hline $\begin{array}{l}\text { Latent-transforming growth factor beta-binding protein } \\
2 \text { (LTBP-2) }\end{array}$ & [22][23] & not found & {$[30]$} \\
\hline Peroxidasin homolog (Vascular peroxidase 1) \# & not found & not found & {$[26]$} \\
\hline $\begin{array}{l}\text { Pigment epithelium-derived factor (PEDF) ( Serpin-F1) } \\
\text { (EPC-1) }\end{array}$ & {$[22][23]$} & {$[26]$} & {$[8][28]$} \\
\hline Plasminogen activator inhibitor 1 (PAI-1) & {$[22][23]$} & {$[24][26]$} & {$[28]$} \\
\hline Prostaglandin-H2 D-isomerase (PGDS2) & [22] & not found & not found \\
\hline Retinol-binding protein 4 (PRBP) (RBP) & {$[22]$} & {$[26]$} & {$[28]$} \\
\hline Sulfhydryl oxidase 1 (hQSOX) & {$[23]$} & [24] & [31] \\
\hline Sushi repeat-containing protein SRPX * & not found & not found & not found \\
\hline Vitamin D-binding protein (DBP) (VDB) \# & not found & not found & [28] \\
\hline \multicolumn{4}{|c|}{ immune regulation } \\
\hline Clusterin (Complement cytolysis inhibitor)(CLI) & {$[22][23]$} & not found & {$[28][30]$} \\
\hline Complement $\mathrm{C} 1 \mathrm{r}$ subcomponent & [23] & {$[24]$} & [30] \\
\hline Complement $\mathrm{C} 1$ s subcomponent & [22][23] & {$[24]$} & [28][30] \\
\hline Complement C3 & [22][23] & not found & {$[8][27]$} \\
\hline $\begin{array}{l}\text { Complement factor D (C3 convertase activator) } \\
\text { (Properdin factor D) (Adipsin) }\end{array}$ & [22][23] & not found & {$[8][28][30]$} \\
\hline $\begin{array}{l}\text { Immunoglobulin superfamily containing leucine-rich } \\
\text { repeat protein * }\end{array}$ & not found & not found & not found \\
\hline Interleukin-25 (IL-25) \# & not found & not found & {$[30]$} \\
\hline Macrophage migration inhibitory factor (MIF) \# & not found & not found & {$[8][28]$} \\
\hline $\begin{array}{l}\text { Pentraxin-related protein PTX3 (Tumor necrosis factor- } \\
\text { inducible gene } 14 \text { protein) }\end{array}$ & {$[22]$} & {$[26]$} & not found \\
\hline Plasma protease C1 inhibitor (C1 Inh) & [22][23] & {$[26]$} & not found \\
\hline \multicolumn{4}{|c|}{ other } \\
\hline $\begin{array}{l}\text { Coiled-coil domain-containing protein } 96 \text { * } \\
\text { Transgelin-2 (SM22-alpha homolog) }\end{array}$ & $\begin{array}{l}\text { not found } \\
{[23]}\end{array}$ & $\begin{array}{l}\text { not found } \\
\text { not found }\end{array}$ & $\begin{array}{l}\text { not found } \\
{[31]}\end{array}$ \\
\hline \multicolumn{4}{|c|}{ not known as secreted proteins } \\
\hline Annexin A5 & not found & not found & [8][27] \\
\hline $\begin{array}{l}\text { Glyceraldehyde-3-phosphate dehydrogenase } \\
\text { (GAPDH) }\end{array}$ & [23] & not found & [31] \\
\hline L-lactate dehydrogenase A chain (LDH-A) & [23] & not found & {$[31]$} \\
\hline $\begin{array}{l}\text { Peptidyl-prolyl cis-trans isomerase A (PPlase A) } \\
\text { (Cyclophilin A) }\end{array}$ & [23] & not found & {$[8][30][31]$} \\
\hline
\end{tabular}




\section{Discussion}

There is a crucial need to study the secretion factors of adipocytes and adipose tissue to finally understand the underlying mechanisms of lipid metabolism, the development of obesity and the obesity-related diseases. In vivo studies are mainly limited to plasma and other body fluids. As a consequence, in vivo studies can demonstrate the result of an effect but not the underlying molecular mechanisms. Therefore, studies of human adipocytes and adipose tissue in vitro are necessary. However, human adipose tissue material is limited with respect to preadipocyte number and differentiation capacity. To avoid this we used SGBS cells, which are morphologically, biochemically and functionally similar to primary preadipocytes and have been used in several other human adipocyte biology studies [16, 37]. However, with these cells profiling of secreted proteins has not been done before. Therefore, a 2-DE-gel approach with/without secretion blocking and a nLC-MALDI-MS/MS approach with bioinformatic analyses were applied to characterize the human secretome during SGBS cell differentiation. Comparison to known proteomic literature demonstrate that the SGBS secretion pattern conforms to the secretion pattern of different primary human and mammal cell material [8, 22-28, 30, 31]. It allowed us to identify 6 novel secreted proteins and 13 proteins which have been described in solely rodent proteomic studies. Complementary literature allowed us to identify 1 protein which is secreted by pig adipose tissue [35]. Therefore, a total of 20 proteins were identified that have not been described in studies with human adipose material. Furthermore, we showed that an additional 23 proteins detected in visceral adipose tissue by others are also secreted by SGBS-cells of subcutaneous origin.

To consider SGBS cells as a valid model for primary human adipocytes the identified secreted proteins were compared with established adipose tissue secreted biomarkes. Leptin, adiponectin, resistin, visfatin, C-C motif chemokine 2 (MCP-1), macrophage migration inhibitory factor (MIF), PAI-1, complement factor D (adipsin), interleukins, tumor necrosis factor alpha (TNF- $\alpha$ ), and retinol-binding protein 4 (RBP-4) are recognized as the most relevant adipose tissue derived factors [38-41]. Of those, none of the human adipose proteomics studies including ours could identify all interleukins, TNF- $\alpha$, MCP-1, leptin, resistin, and visfatin. Only Roelofsen et al. [23] were successful in identifying MCP-1 with a LC-MS/MS approach. Studies in which cytokine arrays were applied could identify interleukins, chemokines and several growth factors [25]. These findings indicate the need for more sensitive techniques like western blotting, ELISA and immuno arrays as additional tools to screen for low-abundant secreted factors. Furthermore, those biomarkers are related to findings in adipose tissue which contains fat cells as well as nonfat cells like blood vessels, macrophages and connective tissue fibroblast. The majority of interleukins and cytokines are released by these nonfat cells and not by pure adipocytes [42]. Still, we were capable to 
identify PAI-1, PEDF, MIF, interleukin-25, adipsin, and RBP-4 as relevant secreted factors as well as other important adipocyte secretion proteins like metalloproteinase inhibitors (TIMP's), 72 kDa type IV collagenase (MMP-2), IBP's, and extracellular matrix proteins like collagens, basement membrane-specific heparan sulfate proteoglycan core protein (HSPG), SPARC, transforming growth factor-beta-induced protein ig-h3 (beta-igH3), and thrombospondin 1 and 2. Previously, Wabitsch et al. [16] detected leptin in SGBS cells by a radioimmunoassay. Even on cellular level we identified adipocyte relevant proteins, which are correlated with lipid metabolism including fatty acid binding proteins, fatty acid synthase, perilipin 4, and enoyl-CoA hyratase (supplement 2). Altogether, this shows that SGBS cells are a reliable model for human adipocyte proteome studies.

For the investigation of the secretion profile of SGBS cells we chose for an experimental set up which included a 2-DE-MS/MS and a nLC-MALDI-MS/MS approach. The 2-DE-MS/MS approach has the advantage of relatively uncomplicated quantification and easy visualization of protein isoforms. We showed that 9 of the 21 secreted proteins such as Collagen alpha$1(\mathrm{I})$ chain and beta ig-h3 are present in several different spots with different intensity and expression pattern. Collagen alpha-1(I) chain was detected in 6 different spots of which only 2 spots were significantly expressed in adipocytes. Some spots for collagens alpha-2(I) and alpha-1(III) were abundantly expressed in adipocytes. Part of the collagen spots and the basement membrane-specific heparan sulfate proteoglycan core protein spot represent the C-peptides of those proteins with molecular weights of $\sim 35 \mathrm{kDa}$ and $22.8 \mathrm{kDa}$, respectively. The presence of these C-peptides demonstrates a significant production and turnover of those proteins. For collagen III our data indicate that production and turnover of this type of collagen is dramatically increased during differentiation. The adipocyte-abundance of procollagen C-endopeptidase enhancer 1 , the enhancer of C-terminal collagen-processing, is in line with this observation. Interestingly, also metalloproteinase inhibitor 1, another moderator of ECM-turnover [43], was detected in 5 spots indicating a complex regulation of its activity by posttranslational modification in preadipocytes and adipocytes. These findings indicate that 2-DE-MS/MS is the preferable proteomic approach to show the existence of different isoforms and their expression behavior. However, the identification of the actual modifications required dedicated mass spectrometry, which is not yet a routine.

2-DE is limited with respect to $\mathrm{pl}$ and mass ranges. Therefore, a sensitive nLC-MALDIMS/MS was used to validate the 2-DE-data and to identify additional adipokines. The application of both methods revealed a broad coverage of the adipokine profiles from SGBS cells. Except for vitamin D-binding protein and retinol-binding protein 4 all other proteins identified with the 2-DE-MS/MS were also detected by the nLC-MALDI-MS/MS method. In this respect, our nLC-MALDI-MS/MS data show only a trend of the expression pattern and the amount of proteins involved. Quantitative conclusions can be made only by linking our 
results with those reported in the literature. Nevertheless, it is remarkable that all proteins which have a functional role in the complement systems (clusterin, complement $\mathrm{C} 1 \mathrm{r}$ subcomponent, complement $\mathrm{C} 1$ s subcomponent, complement $\mathrm{C} 3$, complement factor $\mathrm{D}$, and plasma protease $\mathrm{C} 1$ inhibitor) were found in adipocytes. Chiellini et al., Zvonic et al., and Wang et al. [8, 24, 26] compared in their proteomic studies preadipocyte and adipocyte secretion. In those studies and in our own 2-DE experiment complement C1r subcomponent, complement $\mathrm{C} 1 \mathrm{~s}$ subcomponent, complement $\mathrm{C} 3$, and complement factor $\mathrm{D}$ were either highly or solely secreted by adipocytes. This finding may indicate that proteins with a biological function in the complement systems are particulary secreted by mature adipocytes. Some proteins were identified as secreted that are primarily known as cellular proteins, for instance annexin 5,GAPDH, LDH-A and cyclophilin A. In the literature annexin's function is usually referred to as unknown. Wang et al. [44] documented that annexins are related to stress response, whereas others reported that annexin 5 may play a role in inhibition of blood coagulation by competing for phosphatidylserines $[45,46]$. Phosphatidylserines are part of cell membranes and when a cell undergoes stress that may lead to apoptosis, phosphatidylserine switches to the outside of the membrane [47]. If annexin 5 already interacts with the phosphatidylserine it would pass the membrane and is detected as secreted protein. Yet, there is evidence that annexin 5 might be a non-classical secreted protein, since the SecretomeP NN-score is 0.503 which is higher than the threshold value of 0.5. Cyclophilin $A$ is involved in protein folding and immune regulation. Signal peptide and SecretomeP analyses were negative which explains the SwissProt classification as cytoplasm protein. However, there are several reports in which cyclophilin $A$ is described as secreted protein by a vesicular pathway [48-51] which complies with our present findings. GAPDH and LDH-A have also no signal peptide cleavage sides which indicate them as nonclassical secretion proteins. SecretomeP analysis revealed a NN-score of 0.614 for LDH-A, confirming the secretion characteristics of this protein. According to Swiss-Prot GAPDH is involved in membrane trafficking in the early secretion pathway and is consequently located in the plasma membrane too.

Three proteins of the nLC-MALDI-MS/MS data were not classified by SwissProt as secreted protein. Sushi repeat-containing protein has signal peptide cleavage sites which allow classification as a secreted protein. Its function is rather unclear, but it seems to be involved in cell adhesion and migration [52]. Coiled-coil domain-containing protein 96 and acyl-CoAbinding protein showed no evidence of a signal peptide cleavage site but reached high NNscores on SecretomeP analysis. The function of coiled-coil domain-containing protein 96 is still unknown. Acyl-CoA-binding protein may be an intracellular carrier of acyl-CoA esters. It occurs in 3 isoforms, although the LC-MS/MS data do not allow distinguishing them. All are ubiquitously expressed. In addition, isoform 2 is highly expressed in liver and adipose tissue 
and isoform 3 is strongly expressed in adipose tissue and heart. The $\mathrm{NN}$-scores are 0.546 (isoform 1), 0.553 (isoform 2) and 0.758 (isoform 3), indicating secretory properties. Further, Kinseth et al. [53] classified acyl-CoA-binding protein as a non-classical secreted protein. Altogether these data indicate that acyl-CoA-binding protein is a genuine secreted protein. Correlations between this protein, obesity and diabetes suggest acyl-CoA-binding protein is an adipokine [54, 55].

In this study we identified 6 novel (pre)adipocytes-secreted proteins. Genetic studies suggest that alpha-2HS-glycoprotein is involved in adipocyte insulin action and in the regulation of body composition and insulin sensitivity. This protein is primearily secreted by the liver but identified here as adipocyte-secreted protein [56, 57].

Lysyl oxidase homolog 2, another protein that is identified here may play a role in remodeling of adipocyte structure during adipogenesis. During differentiation cells undergo enormous structural changes, including remodeling of extracellular matrix proteins like collagens. The lysyl oxidase is involved in crosslinking collagens and elastins. Furthermore, lysyl oxidase homolog 2 is associated with extracellular matrix remodeling during fibrotic disorders [58]. Glia-derived nexin belongs to the serine protease inhibitor superfamily and is usually related to neurobiological processes [59,60]. Known adipokines such as plasminogen activator inhibitor 1 (PAI-1) and pigment epithelium-derived factor (PEDF) are also members of the serine protease inhibitor superfamily $[8,24]$. Similar to glia-derived nexin their biological roles are not directly associated to the adipocyte biology. According to SwissProt PAI-1 is an inhibitor and decoy for tissue plasminogen activator, urokinase, and protein $\mathrm{C}$. It seems that PAI-1 is a major control point in the regulation of fibrinolysis. PEDF is a neurotrophic protein and it induces extensive neuronal differentiation in retinoblastoma cells. In addition, PEDF is a potent inhibitor of angiogenesis. The relationship to these known adipokines suggests that glia-derived nexin actions might not be limited to neurobiological processes.

Immunoglobulin superfamily containing leucine-rich repeat protein contains a leucine-rich repeat domain (LRR) and an immunoglobulin-like domain $\left(\lg C_{2}\right)$, which is mostly expressed in the nervous systems. However, immunoglobulin superfamily containing leucine-rich repeat protein is not only expressed in the nervous system but in various other tissues such as heart, skeletal muscle, testis, and retina. Its exact functions in these tissues remain unclear. More and more transmembrane proteins are found that contains both domains. Therefore, this protein may play a role in protein-protein interactions and cell-adhesion [61, 62].

Similar to immunoglobulin superfamily containing leucine-rich repeat protein the functions and the exact role of sushi repeat-containing protein and coiled-coil domain-containing protein 96 remains unknown. Further investigations of these proteins are necessary to distinguish their functional properties. 


\section{Conclusion}

Altogether, our study shows that SGBS cells with their ideal culturing and manipulation qualities behave as a proper cell model for adipocyte-related proteomics studies. This is further validated by the high conformance of identified secretion proteins between SGBS and other adipocyte cell strains, by the presence of adipose tissue-specific biomarkers as adiponectin and leptin, as well as by the detection of typical adipocyte cellular proteins. Although confirmation may be needed from primary human cells, our present results show that SGBS cells can be readily used to identify novel human adipocyte secretion factors, to demonstrate in human the presence of secretion factors identified in other species, and to ascribe identified adipocyte secreted proteins to subcutaneous adipose tissue.

\section{Acknowledgements}

We thank Dr. Ping Wang of the Department of Human Biology, Maastricht University for practical knowledge and helpful discussions.

\section{References}

1. Dixon, J.B., The effect of obesity on health outcomes. Mol Cell Endocrinol, 2010. 316(2): p. 104-8.

2. Runge, C.F., Economic consequences of the obese. Diabetes, 2007. 56(11): p. 266872.

3. Wang, Y., et al., Will all Americans become overweight or obese? estimating the progression and cost of the US obesity epidemic. Obesity (Silver Spring), 2008. 16(10): p. 2323-30.

4. Diamanti-Kandarakis, E., et al., The Impact of Endocrine Disruptors on Endocrine Targets. Horm Metab Res, 2010. 42(8): p. 543-52.

5. Gregoire, F.M., Adipocyte differentiation: from fibroblast to endocrine cell. Exp Biol Med (Maywood), 2001. 226(11): p. 997-1002.

6. Goossens, G.H., The role of adipose tissue dysfunction in the pathogenesis of obesity-related insulin resistance. Physiol Behav, 2008. 94(2): p. 206-18.

7. Mohamed-Ali, V., J.H. Pinkney, and S.W. Coppack, Adipose tissue as an endocrine and paracrine organ. Int J Obes Relat Metab Disord, 1998. 22(12): p. 1145-58.

8. Wang, P., et al., Profiling of the secreted proteins during 3T3-L1 adipocyte differentiation leads to the identification of novel adipokines. Cell Mol Life Sci, 2004. 61(18): p. 2405-17. 
9. Chen, $\mathrm{H}_{\text {., }}$ et al., Evidence that the diabetes gene encodes the leptin receptor: identification of a mutation in the leptin receptor gene in $\mathrm{db} / \mathrm{db}$ mice. Cell, 1996. 84(3): p. 491-5.

10. Himms-Hagen, J., Physiological roles of the leptin endocrine system: differences between mice and humans. Crit Rev Clin Lab Sci, 1999. 36(6): p. 575-655.

11. Kadowaki, T. and T. Yamauchi, Adiponectin and adiponectin receptors. Endocr Rev, 2005. 26(3): p. 439-51.

12. Patel, L., et al., Resistin is expressed in human macrophages and directly regulated by PPAR gamma activators. Biochem Biophys Res Commun, 2003. 300(2): p. 472-6.

13. Rosen, B.S., et al., Adipsin and complement factor D activity: an immune-related defect in obesity. Science, 1989. 244(4911): p. 1483-7.

14. Steppan, C.M., et al., The hormone resistin links obesity to diabetes. Nature, 2001. 409(6818): p. 307-12.

15. Wang, P., et al., The secretory function of adipocytes in the physiology of white adipose tissue. J Cell Physiol, 2008. 216(1): p. 3-13.

16. Wabitsch, M., et al., Characterization of a human preadipocyte cell strain with high capacity for adipose differentiation. Int J Obes Relat Metab Disord, 2001. 25(1): p. 815.

17. Nickel, W., The mystery of nonclassical protein secretion. A current view on cargo proteins and potential export routes. Eur J Biochem, 2003. 270(10): p. 2109-19.

18. Orci, L., et al., Brefeldin A, a drug that blocks secretion, prevents the assembly of non-clathrin-coated buds on Golgi cisternae. Cell, 1991. 64(6): p. 1183-95.

19. Bouwman, F., J. Renes, and E. Mariman, A combination of protein profiling and isotopomer analysis using matrix-assisted laser desorption/ionization-time of flight mass spectrometry reveals an active metabolism of the extracellular matrix of 3T3-L1 adipocytes. Proteomics, 2004. 4(12): p. 3855-63.

20. Cranenburg, E.C., et al., The circulating inactive form of matrix Gla Protein (ucMGP) as a biomarker for cardiovascular calcification. J Vasc Res, 2008. 45(5): p. 427-36.

21. Dumont, D., et al., Characterization of mature rat oligodendrocytes: a proteomic approach. J Neurochem, 2007. 102(2): p. 562-76.

22. Alvarez-Llamas, G., et al., Characterization of the human visceral adipose tissue secretome. Mol Cell Proteomics, 2007. 6(4): p. 589-600.

23. Roelofsen, $\mathrm{H}_{\text {., }}$ et al., Comparison of isotope-labeled amino acid incorporation rates (CILAIR) provides a quantitative method to study tissue secretomes. Mol Cell Proteomics, 2009. 8(2): p. 316-24.

24. Chiellini, C., et al., Characterization of human mesenchymal stem cell secretome at early steps of adipocyte and osteoblast differentiation. BMC Mol Biol, 2008. 9: p. 26. 
25. Sell, H., et al., Cytokine secretion by human adipocytes is differentially regulated by adiponectin, AICAR, and troglitazone. Biochem Biophys Res Commun, 2006. 343(3): p. $700-6$.

26. Zvonic, S., et al., Secretome of primary cultures of human adipose-derived stem cells: modulation of serpins by adipogenesis. Mol Cell Proteomics, 2007. 6(1): p. 18-28.

27. Aoki, N., et al., Identification and characterization of microvesicles secreted by 3T3L1 adipocytes: redox- and hormone-dependent induction of milk fat globule-epidermal growth factor 8-associated microvesicles. Endocrinology, 2007. 148(8): p. 3850-62.

28. Chen, X., et al., Quantitative proteomic analysis of the secretory proteins from rat adipose cells using a 2D liquid chromatography-MS/MS approach. J Proteome Res, 2005. 4(2): p. 570-7.

29. Kratchmarova, I., et al., A proteomic approach for identification of secreted proteins during the differentiation of 3T3-L1 preadipocytes to adipocytes. Mol Cell Proteomics, 2002. 1(3): p. 213-22.

30. Lim, J.M., et al., Defining the regulated secreted proteome of rodent adipocytes upon the induction of insulin resistance. J Proteome Res, 2008. 7(3): p. 1251-63.

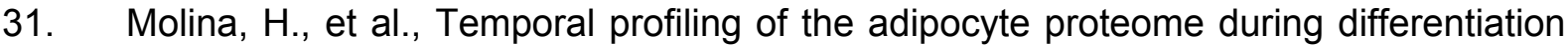
using a five-plex SILAC based strategy. J Proteome Res, 2009. 8(1): p. 48-58.

32. Hossner, K.L., et al., Insulin-like growth factor (IGF)-I and -II and IGFBP secretion by ovine satellite cell strains grown alone or in coculture with 3T3-L1 preadipocytes. In Vitro Cell Dev Biol Anim, 1997. 33(10): p. 791-5.

33. Wabitsch, M., et al., IGF-I- and IGFBP-3-expression in cultured human preadipocytes and adipocytes. Horm Metab Res, 2000. 32(11-12): p. 555-9.

34. Baxter, R.C. and S.M. Twigg, Actions of IGF binding proteins and related proteins in adipose tissue. Trends Endocrinol Metab, 2009. 20(10): p. 499-505.

35. Hausman, G.J., et al., Secreted proteins and genes in fetal and neonatal pig adipose tissue and stromal-vascular cells. J Anim Sci, 2006. 84(7): p. 1666-81.

36. Kallio, P., et al., Association of sequence variations in the gene encoding insulin-like growth factor binding protein 5 with adiponectin. Int $\mathrm{J}$ Obes (Lond), 2009. 33(1): p. 80-8.

37. Fischer-Posovszky, P., et al., Human SGBS Cells - a Unique Tool for Studies of Human Fat Cell Biology. Obes Facts, 2008. 1(4): p. 184-189.

38. Hauner, H., Secretory factors from human adipose tissue and their functional role. Proc Nutr Soc, 2005. 64(2): p. 163-9.

39. Karastergiou, K. and V. Mohamed-Ali, The autocrine and paracrine roles of adipokines. Mol Cell Endocrinol, 2010. 318(1-2): p. 69-78. 
40. Trayhurn, P., C. Bing, and I.S. Wood, Adipose tissue and adipokines--energy regulation from the human perspective. J Nutr, 2006. 136(7 Suppl): p. 1935S-1939S.

41. Vazquez-Vela, M.E., N. Torres, and A.R. Tovar, White adipose tissue as endocrine organ and its role in obesity. Arch Med Res, 2008. 39(8): p. 715-28.

42. Fain, J.N., Release of interleukins and other inflammatory cytokines by human adipose tissue is enhanced in obesity and primarily due to the nonfat cells. Vitam Horm, 2006. 74: p. 443-77.

43. Mariman, E.C. and P. Wang, Adipocyte extracellular matrix composition, dynamics and role in obesity. Cell Mol Life Sci, 2010. 67(8): p. 1277-92.

44. Wang, P., F.G. Bouwman, and E.C. Mariman, Generally detected proteins in comparative proteomics--a matter of cellular stress response? Proteomics, 2009. 9(11): p. 2955-66.

45. Rand, J.H., et al., Resistance to annexin A5 anticoagulant activity: a thrombogenic mechanism for the antiphospholipid syndrome. Lupus, 2008. 17(10): p. 922-30.

46. Urso, B., et al., Comparison of anti-apoptotic signalling by the insulin receptor and IGF-I receptor in preadipocytes and adipocytes. Cell Signal, 2001. 13(4): p. 279-85.

47. Fink, S.L. and B.T. Cookson, Apoptosis, pyroptosis, and necrosis: mechanistic description of dead and dying eukaryotic cells. Infect Immun, 2005. 73(4): p. 1907-16.

48. Jin, Z.G., et al., Cyclophilin A is a secreted growth factor induced by oxidative stress. Circ Res, 2000. 87(9): p. 789-96.

49. Jin, Z.-G., et al., Cyclophilin A Is a Proinflammatory Cytokine that Activates Endothelial Cells. Arterioscler Thromb Vasc Biol, 2004. 24(7): p. 1186-1191.

50. Sherry, B., et al., Identification of cyclophilin as a proinflammatory secretory product of lipopolysaccharide-activated macrophages. Proc Natl Acad Sci U S A, 1992. 89(8): p. 3511-5.

51. Suzuki, J., et al., Cyclophilin A Is Secreted by a Vesicular Pathway in Vascular Smooth Muscle Cells. Circ Res, 2006. 98(6): p. 811-817.

52. Tanaka, K., et al., SRPX2 is overexpressed in gastric cancer and promotes cellular migration and adhesion. Int J Cancer, 2009. 124(5): p. 1072-80.

53. Kinseth, M.A., et al., The Golgi-associated protein GRASP is required for unconventional protein secretion during development. Cell, 2007. 130(3): p. 524-34.

54. Ejaz, A., et al., Curcumin inhibits adipogenesis in 3T3-L1 adipocytes and angiogenesis and obesity in C57/BL mice. J Nutr, 2009. 139(5): p. 919-25.

55. Zhou, X.R., et al., Dietary conjugated linoleic acid increases PPAR gamma gene expression in adipose tissue of obese rat, and improves insulin resistance. Growth Horm IGF Res, 2008. 18(5): p. 361-8. 
56. Dahlman, I., et al., alpha2-Heremans-Schmid glycoprotein gene polymorphisms are associated with adipocyte insulin action. Diabetologia, 2004. 47(11): p. 1974-9.

57. IX, J.H. and K. Sharma, Mechanisms linking obesity, chronic kidney disease, and fatty liver disease: the roles of fetuin-A, adiponectin, and AMPK. J Am Soc Nephrol, 2010. 21(3): p. 406-12.

58. Decitre, M., et al., Lysyl oxidase-like protein localizes to sites of de novo fibrinogenesis in fibrosis and in the early stromal reaction of ductal breast carcinomas. Lab Invest, 1998. 78(2): p. 143-51.

59. Gettins, P.G., Serpin structure, mechanism, and function. Chem Rev, 2002. 102(12): p. 4751-804.

60. Silverman, G.A., et al., The serpins are an expanding superfamily of structurally similar but functionally diverse proteins. Evolution, mechanism of inhibition, novel functions, and a revised nomenclature. J Biol Chem, 2001. 276(36): p. 33293-6.

61. Nagasawa, A., et al., Cloning of the cDNA for a new member of the immunoglobulin superfamily (ISLR) containing leucine-rich repeat (LRR). Genomics, 1997. 44(3): p. 273-9.

62. Wu, M., et al., LRRC4 controls in vitro invasion of glioblastoma cells through inhibiting RPTP-zeta expression. J Neurooncol, 2006. 80(2): p. 133-42. 


\section{Chapter 4}

\section{Expression of functional tissue factor by human preadipocytes and adipocytes}

Anja Rosenow, Simone J. H. Wielders, Edouard M. Bevers, Martin Wabitsch, Theo Lindhout, Edwin C.M. Mariman, Johan Renes

submitted 


\section{CHAPTER 4}

\section{Abstract}

Adipose tissue is an endocrine organs and its para- and endocrine function is tight-regulated. Altered secretion patterns due to excessive adipose tissue growth during obesity are involved in the initiation of metabolic diseases including insulin resistance, type 2 diabetes, cardiovascular diseases and several cancers. Tissue factor (TF) expression during adipose tissue expansion could be a major contributor of intravascular thrombosis and extravascular fibrosis of obese subjects. However, next to its strict blood coagulation function, TF is also known to be involved in intracellular signaling pathways and angiogenesis. Most studies on TF expression in adipose tissue have been done on mRNA/gene levels of rodents. Here, we investigated the expression and activity of TF from preadipocytes and adipocytes of human subcutaneous Simpson-Golabi-Behmel syndrome (SGBS) fat cells. TF expression was detected on SGBS (pre)adipocytes and in the medium derived from these cells. In addition, TF-mediated factor $\mathrm{Xa}$ and thrombin activation was observed both on the cells and in the medium. Our data demonstrate that human (pre)adipocytes express functional TF, which may have implications for obesity-induced thrombosis, extravascular fibrosis and neovascularisation. 


\section{Introduction}

Adipose tissue is one of the largest human endocrine organs and its para- and endocrine function is tightly regulated. Adipose tissue-secreted proteins affect the overall metabolism as well as that of many organs and tissues. During obesity, the number of adipocytes (hyperplasia) as well as the cell size of existing adipocytes increases (hypertrophy) both leading to an excessive growth of adipose tissue [1,2]. In addition, adipocytes of obese subjects are subjected to hypoxic conditions due to limited nutrition and oxygen supplies from the blood-stream [3-5]. Both, excessive adipose tissue growth and the resulting hypoxic conditions of adipocytes, result in altered secretion patterns of the adipose tissue. Such dysfunction may be the initiator of the obesity-associated diseases including insulin resistance, type 2 diabetes, cardiovascular diseases and several cancers [6, 7].

Tissue factor (TF) is expressed by many extravascular cells, including fibroblasts, which have no direct contact to the blood stream. TF is mostly known as initiator of the extrinsic pathway of the blood coagulation by activating factor VII [8, 9]. As such, increased TF expression during excessive growth of adipose tissue could be a major contributor of intravascular thrombosis and extravascular fibrosis of obese subjects [10-12]. However, next to its strict blood coagulation function, TF is also involved in intracellular signaling pathways [13] and angiogenesis [8, 14]. The intracellular signaling of cell membrane glycoproteins such as TF is also linked to protease-activated receptor 1 (PAR-1) and PAR-2 [15]. TF-PAR2 signaling within adipocytes negatively affects weight gain, insulin resistance and inflammation [16]. On the other hand, TF-PAR2 signaling of fast growing tumor tissue is associated with tumor angiogenesis by inducing pro-angiogenic factors [17, 18]. There are at least 5 alternatively spliced Tissue Factors (asTF) with differential expression and activity levels [19-21]. As such, asTF may be non-coagulative but promote angiogenesis processes [22].

Most studies on TF expression in adipose tissue have been done on mRNA/gene levels of rodents $[11,12,16,23]$. Levels of TF mRNA do not necessarily reflect TF activity because of different expression of protein isoforms. In addition, the majority of cell bound TF on cell surfaces is inactive. Furthermore, the presence of anionic phospholipids, in particular phosphatidylserine (PS) is crucial for the action of cell surface expressed TF as a cofactor of the clotting system [9]. TF expression during differentiation of human preadipocytes has not been demonstrated yet. Presence of active TF on human adipocytes may have implications for obesity-associated thrombosis, extravascular fibrosis and angiogenesis. Therefore, we investigated here the expression and activity of TF from preadipocytes and adipocytes of human subcutaneous Simpson-Golabi-Behmel syndrome (SGBS) fat cells [24, 25]. 


\section{Methods}

\section{Material}

Culture media, $0.5 \%$ trypsin-EDTA and $10,000 \mathrm{u} / \mathrm{ml}$ Penicillin together with $10,000 \mathrm{u} / \mathrm{ml}$ Streptomycin solutions were obtained from Invitrogen (Carlsbad, CA. USA). Fetal bovine serum (FBS) was obtained from Bodinco (Alkmaar, The Netherlands). Additional cell culture supplements, protease inhibitor cocktail, DL-Dithiothreitol (DTT), 3-[(3-cholamidopropyl) dimethyl-amonio]-1-propanesulfonate (CHAPS) were purchased from Sigma-Aldrich (Zwijndrecht, The Netherlands).

\section{Cell culture}

Human Simpson-Golabi-Behmel syndrome (SGBS) cells were obtained from Prof. Dr. M. Wabitsch (University of Ulm, Germany) [25]. Preadipocytes were seeded in low concentration $\left(2 \times 10^{4}\right.$ cells) in wells of a well plate and cultured up to $90 \%$ confluence in $10 \%$ FBS-containing DMEM/F12 medium supplemented with $66 \mathrm{nM}$ biotin, $33 \mathrm{nM}$ D-pantothenic acid and $1 \%$ penicillin/streptomycin antibiotics. Every 2 to 3 days the cells were washed with PBS buffer and medium was changed. Number of cells per 48-well was $52 \pm 9 \times 10^{3}$ (mean \pm $\mathrm{SD}, \mathrm{n}=5)$.

\section{Induction of Differentiation}

To induce differentiation, confluent preadipocytes were washed twice with PBS buffer and the medium was changed to serum free DMEM/F12 medium containing $10 \mu \mathrm{g} / \mathrm{mL}$ human transferrin, $20 \mathrm{nM}$ insulin, $100 \mathrm{nM}$ cortisol and $200 \mathrm{pM}$ triiodothyronine, $500 \mu \mathrm{M}$ 1-methyl-3isobutyl-xanthine, $25 \mathrm{nM}$ dexamethasone and $2 \mu \mathrm{M}$ rosiglitazone for the first $96 \mathrm{~h}$. Subsequently the cells were further differentiated in serum free DMEM/F12 medium containing $10 \mu \mathrm{g} / \mathrm{mL}$ human transferrin, $20 \mathrm{nM}$ insulin, $100 \mathrm{nM}$ cortisol and $200 \mathrm{pM}$ triiodothyronine. Every 2 to 3 days the medium was refreshed without washing the cells. After 14 days, up to $80 \%$ of the preadipocytes were differentiated into mature adipocytes. The number of adipocytes per well after rinsing with HEPES buffer prior to experimentation was $14.9 \pm 3.5 \times 10^{3}$ (mean $\left.\pm S D, n=14\right)$.

\section{Measurement of TF by Western blotting}

Cellular and secretion material were obtained from $1.6 \pm 1.3 \times 10^{6}$ (mean $\pm S D, n=4$ ) adipocytes and $2.2 \pm 0.4 \times 10^{6}$ (mean $\pm S D, n=3$ ) preadipocytes. The media for obtaining secretion protein material were collected, supplemented with protease inhibitor cocktail, transferred to dialysis tubes (2-kDa molecular-weight cut-off, Carl Roth $\mathrm{GmbH}$, Karlsruhe, Germany) and dialyzed against $20 \mathrm{mM}$ ammonium bicarbonate at $4^{\circ} \mathrm{C}$ for $48 \mathrm{~h}$ with $10-12$ buffer changes. The dialyzed medium samples were freeze-dried, dissolved in fresh 
rehydration buffer ( $8 \mathrm{M}$ urea, $2 \% \mathrm{w} / \mathrm{v}$ CHAPS and $65 \mathrm{mM} \mathrm{DTT}$ ) and centrifuged at $20000 \mathrm{x} \mathrm{g}$ and $10^{\circ} \mathrm{C}$ for $30 \mathrm{~min}$. To obtain cellular protein material cells were solubilised in lysis buffer consisting of $8 \mathrm{M}$ urea, $2 \% \mathrm{w} / \mathrm{v}$ CHAPS and $65 \mathrm{mM}$ DTT and a protease inhibitor cocktail. The protein concentrations were estimated by a Bradford-based protein assay kit (BioRad Laboratories, Veenendaal, The Netherlands).

$30 \mathrm{ug}$ of the protein solutions were separated by $12 \%$ Criterion TGX electrophoresis gels (BioRad) and then transferred to a nitrocellulose membrane (Trans-Blot Turbo Transfer Pack, BioRad). The membrane was blocked with $5 \%$ milk and probed with a primary mouse monoclonal anti-human TF antibody (R\&D Systems, Abington, UK) or mouse $\beta$-actin (Santa Cruz, Biotechnology, Heidelberg, Germany). Afterwards, the membrane was subjected to secondary anti-mouse antibodies (DAKO, Glostrup, Denmark). The specific proteins were visualized with the enhanced chemiluminescence reagent SuperSignal west Dura extended duration substrate (Pierce, Thermo Fisher Scientific, Etten-Leur, The Netherlands). $\beta$-actin adjusted volume levels were used to normalize for sample fluctuations.

\section{Measurement of TF activity on cell surfaces and in conditioned medium}

TF activity expressed at cell membrane surfaces was measured through its ability to catalyze the factor VIla-mediated activation of factor X. Cells grown in 48-well plates were washed with HEPES buffer (10 mM HEPES, $136 \mathrm{mM} \mathrm{NaCl}, 2 \mathrm{mM} \mathrm{MgCl}, 2.7 \mathrm{mM} \mathrm{KCl}, 1 \mathrm{mg} / \mathrm{mL}$ glucose, and $1 \mathrm{mg} / \mathrm{mL}$ bovine serum albumin, $\mathrm{pH} 7.5$ ), and then incubated during $5 \mathrm{~min}$ with $270 \mu \mathrm{L}$ HEPES buffer containing $0.11 \mathrm{nM}$ factor VIla, $3.3 \mathrm{mM} \mathrm{CaCl}_{2}, 0.22 \mathrm{mM}$ Pefafluor factor Xa substrate (Pentapharm, Basel, Switzerland) and when applicable with polyclonal antihuman TF IgG antibody (TF-5, Sanguin, Amsterdam, The Netherlands) TF antibodies and annexin A5 (MosaMedix, Kattendrijke, The Netherlands). In neutralizing experiments with TF or annexin A5, the incubation time was $15 \mathrm{~min}$. The reaction was started by the addition of $30 \mu \mathrm{L}$ HEPES buffer containing $1 \mu \mathrm{M}$ factor $\mathrm{X}$ and fluorescence tracings were recorded in a plate spectrofluorometer (SpectraMax M2, Molecular Devices, Sunnyvale, CA, USA) at $37^{\circ} \mathrm{C}$ with an excitation and emission wavelength of $350 \mathrm{~nm}$ and $450 \mathrm{~nm}$, respectively. To obtain initial rates of factor Xa generation, the slopes of the first derivatives of the fluorescence tracings were calculated. Known amounts of purified factor Xa were used to construct a reference line. For the measurement of TF activity in (pre)adipocyte-derived culture medium, $100 \mu \mathrm{L}$ conditioned medium, exposed for $24 \mathrm{~h}$ to the cell cultures, was mixed with $35 \mu \mathrm{l}$ HEPES buffer containing $0.43 \mathrm{nM}$ factor VIla, $13 \mathrm{mM} \mathrm{CaCl}_{2}$ and $0.86 \mathrm{mM}$ Pefafluor factor Xa substrate in a 96-well plate. The reaction was started with $15 \mu \mathrm{L}$ HEPES buffer containing 1 $\mu \mathrm{M}$ factor $\mathrm{X}$. TF activity was corrected for a small contribution of substrate converting activity in serum-containing preadipocyte medium. All procedures were performed at $37^{\circ} \mathrm{C}$. 


\section{CHAPTER 4}

\section{Plasma preparation}

Blood was drawn from healthy volunteers by puncture of antecubital vein and freely drained on 0.1 vol trisodium citrate $(0.13 \mathrm{M})$. Platelet free plasma (PFP) was prepared from citrated blood by centrifugation twice at $870 \mathrm{~g}$ during $10 \mathrm{~min}$ at $20^{\circ} \mathrm{C}$, followed by centrifugation at $40,000 \mathrm{~g}$ during $60 \mathrm{~min}$ at $4^{\circ} \mathrm{C}$.

\section{Determination of thrombin generation on cell surfaces and in conditioned medium}

Preadipocytes and mature adipocytes were grown in 48-well plates, rinsed with HEPES buffer and then incubated with $250 \mu \mathrm{L}$ platelet-free plasma (PFP) containing $480 \mu \mathrm{M}$ of the thrombin fluorogenic substrate Z-Gly-Gly-Arg-AMC.HCl (ZGGR-AMC; Bachem, Weil am Rhein, Germany), $60 \mu \mathrm{g} / \mathrm{mL}$ corn trypsin inhibitor (CTI; Hematologic Technologies, Essex Junction, VT, USA) and when applicable, TF antibodies or annexin A5. Thrombin generation was started by addition of $50 \mu \mathrm{L}$ HEPES buffer containing $100 \mathrm{mM} \mathrm{CaCl}_{2}$. Fluorescence was measured with $\lambda_{\mathrm{exc}}=368 \mathrm{~nm}$ and $\lambda_{\mathrm{em}}=460 \mathrm{~nm}$ using a SpectraMax M2 plate reader. Fluorescence tracings were converted into thrombin concentrations as described [26]. The maximal rate of thrombin generation during the propagation phase (maxR) was calculated by linear regression. For the measurement of medium-induced thrombin generation, $60 \mu \mathrm{L}$ conditioned (24 h) medium was mixed with $60 \mu \mathrm{L}$ PFP in a 96-well plate. The reaction was started with $20 \mu \mathrm{L}$ HEPES buffer containing ZGGR-AMC and $\mathrm{CaCl}_{2}$. The final concentrations of reactants and fluorescence measurements were similar to that described for the thrombin generation assay with cell surfaces. All procedures were performed at $37^{\circ} \mathrm{C}$.

\section{Statin and mevalonate intervention}

Preadipocytes and mature adipocytes grown in 48-well plates were incubated for $24 \mathrm{~h}$ with medium containing increasing concentrations of simvastatin (0-10 $\mu \mathrm{M}$, Merck, Darmstadt, Germany) or medium containing $1 \mu \mathrm{M}$ simvastatin and increasing amounts of mevalonolactone (0-100 $\mu \mathrm{M}$; Sigma-Aldrich, Zwijndrecht, The Netherlands). Media were removed and cells were washed with HEPES buffer. TF activity in conditioned medium and cell-bound TF activity were measured as described before.

\section{Statistical analysis}

Results are expressed as mean \pm S.D. Differences between groups of the activity measurements were evaluated by the non-parametric Mann-Whitney $U$ test. Differences between groups were considered statistical significant when $p<0.05$. Western blotting differences between pre- and adipocytes were analyzed by the Student's T-test and considered as statistical significant when $p<0.05$. 


\section{Results}

\section{Detection of TF by Western Blotting}

Cellular and secretion proteins from preadipocytes and adipocytes were used to demonstrate TF expression on both cell types. Cellular TF expression results were normalized against $\beta$ actin expression. The analyses of cellular and secretion material showed two protein bands around 70 to $75 \mathrm{kDa}$ (Figure 1). The masses of these bands are neither according to the theoretical molecular mass of $33 \mathrm{kDa}$ (isoform 1, aa 1 to 295) nor to the post-translational modified molecular mass of $47 \mathrm{kDa}$ [27]. Use of a different rabbit polyclonal TF antibody (Abcam, Cambridge, UK) confirmed our findings with abundant bands around 70 to $75 \mathrm{kDa}$ (data not shown). To investigate the possible tissue- or species-specificity of the observed protein bands in the SGBS cells, we examined TF expression by the same antibody in samples of different tissue origin including subcutaneous adipose tissue, mouse kidney and brain, human muscle, HEPG2 and human cervical Hela cells. The expected protein band of around $47 \mathrm{kDa}$ was detected in mouse brain and kidney cells. Subcutaneous adipose tissue expressed specific bands around $50 \mathrm{kDa}$ and $75 \mathrm{kDa}$. Within HEPG2, Hela and human muscle cells no TF was detectable (Figure 1C). Apparently, TF can occur in various alternative forms in human cells.

Compared to adipocytes, TF expression in cellular material from preadipocytes is significantly higher $(9$-fold, $p<0.05)$. With respect to TF expression in secreted protein material a slightly, but not significant, increased level was observed with preadipocytes compared to adipocytes. 
A

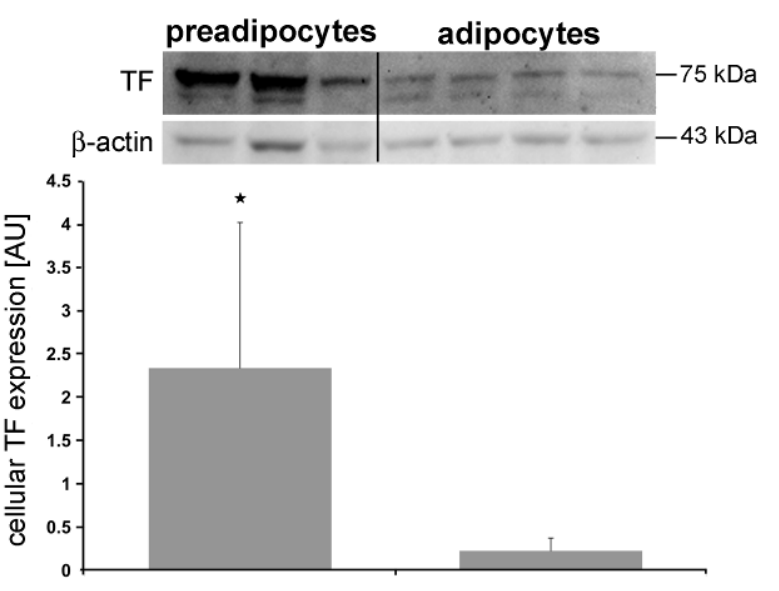

B

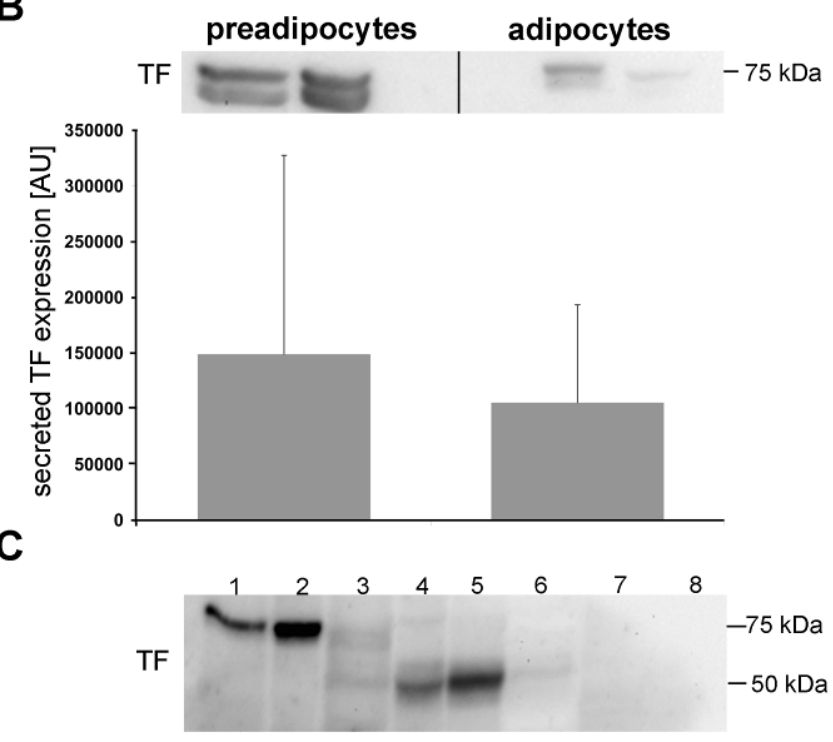

Figure 1: TF expression and secretion levels in preadipocyte and adipocytes.

Western blotting analysis was performed for TF protein with $30 \mu \mathrm{g}$ of total protein extracts. A: Cellular expression of TF. The $\beta$-actin protein signal was used for normalization. Reported values of preadipocytes are the means with SD of 3 biological replicates and of adipocytes are the means of 4 biological replicates. * indicates significant differences with $p<$ 0.05 . B: Secreted expression of TF. No internal protein standards for normalization are available and therefore no significant statistics could be performed. Reported values of preadipocytes and adipocytes are the means of 3 biological replicates. C: TF expression from different cell origin, 1 : SGBS adipocytes, 2: SGBS preadipocytes, 3: subcutaneous adipose tissue, 4: mouse brain, 5: mouse kidney, 6: human muscle, 7: HEPG2 cells, 8: Hela cells.

\section{Constitutive functional TF on preadipocytes and mature adipocytes}

For further investigations of the Western blot data, we performed functional TF assays with the SGBS cells. Cultures of preadipocytes and mature adipocytes $\left(52 \times 10^{3}\right.$ preadipocytes and $15 \times 10^{3}$ adipocytes) were exposed to a mixture of factor VIla and factor $X$ to measure TF activity. Preadipocytes as well as adipocytes express TF activity at their surfaces (Figure $2 A)$. As a reference, the rate of factor Xa generation $(0.456 \mathrm{~nm} / \mathrm{min})$ in the presence of $1 \mathrm{pM}$ recombinant TF (rhTF; Innovin; Dade Behring, Marburg, Germany) under similar conditions, but in the absence of cells was measured. After normalization for cell number differences and rhTF reference, adipocytes show an 8-fold higher TF activity compared to preadipocytes. We noted that the estimated cell-associated TF activity is based on initial rates of factor Xa generation using a commercial TF preparation of which the molar concentration was established by titration with purified human recombinant factor VIla (Novo Nordisk, Alphen aan de Rijn, The Netherlands). That indeed factor Xa generation was mediated by TF could be concluded from the neutralizing activity of a polyclonal anti-human TF IgG (TF-5; Sanguin, Amsterdam, The Netherlands). TF-5 $(20 \mu \mathrm{g} / \mathrm{mL})$ reduced the rate of factor Xa generation on 
preadipocytes and adipocytes with $92 \%$ and $86 \%$, respectively. In addition, annexin A5 (0.5 $\mu \mathrm{M}$; MosaMedix, Kattendijke, The Netherlands), a protein with high affinity for anionic phospholipids, inhibited the rate of factor Xa generation with $63 \%$ and $71 \%$, respectively. Together, these results indicate that factor $X$ activation on preadipocytes and mature adipocytes is driven by TF and that anionic phospholipids are partially involved in this reaction, because a saturating amount of annexin A5 [28], did not fully block cell-bound TF activity.

A

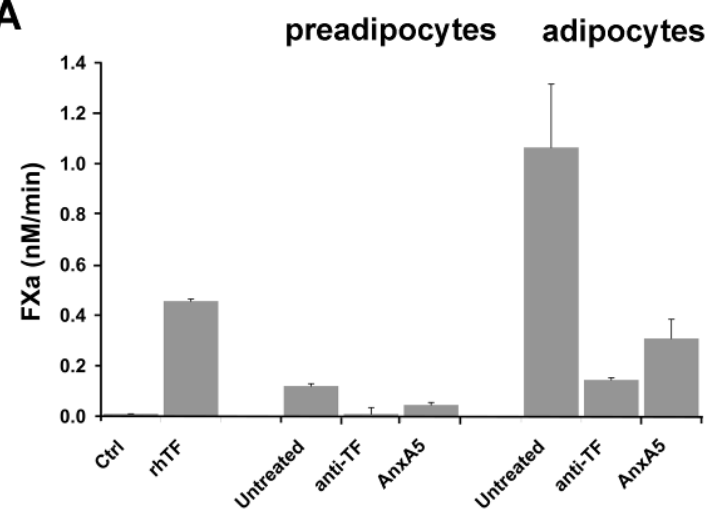

B

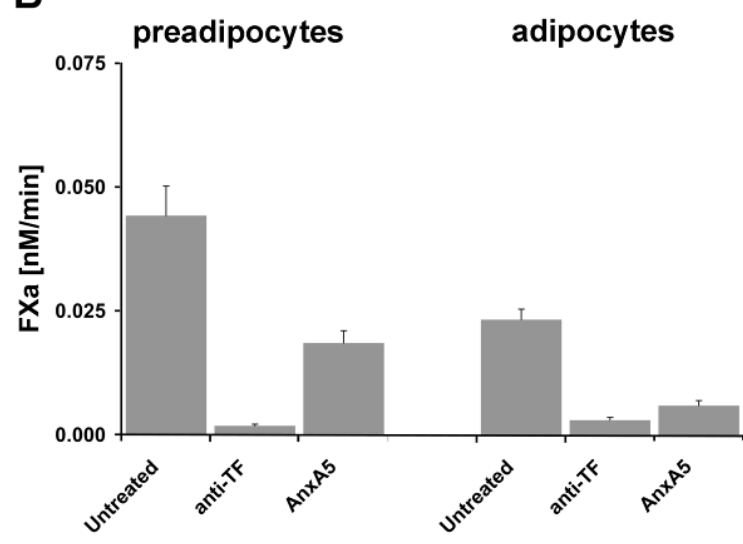

Figure 2: Constitutive expression of TF activity. A: factor Xa generation on preadipocytes and mature adipocytes grown in 48-well plate in the absence (Untreated) and presence of polyclonal rabbit antihuman TF IgG (anti-TF) and annexin A5 (AnxA5). Twenty four hours prior to the FX activation assay, the cell culture medium was refreshed. As references, the rate of factor Xa generation in an empty well $(\mathrm{Ctrl})$ and the rate in the presence of 1 pM recombinant TF (rhTF) are shown. B: samples $(0.1 \mathrm{~mL})$ were taken from medium that was removed prior to the factor $X$ activation assay described under $A$ and transferred to a 96 wellplate to measure factor $\mathrm{Xa}$ generation in the absence (Untreated) and presence of polyclonal rabbit anti-human TF IgG (anti-TF) and annexin A5 (AnxA5) as described under Methods. All experiments were performed at $37^{\circ} \mathrm{C}$. Both data sets are normalized for number of cells

Medium that was removed from the cell cultures $\left(52 \times 10^{3}\right.$ preadipocytes and $15 \times 10^{3}$ adipocytes), prior to the measurement of surface-bound TF was also analyzed for the presence of TF activity. We found that preadipocytes and mature adipocytes shed factor $X$ converting activity into their media. As such, TF concentrations in the media after normalization for cell count were $0.043 \mathrm{nM} / \mathrm{min}$ and $0.022 \mathrm{nM} / \mathrm{min}$, respectively (Figure $2 \mathrm{~B}$ ). The preadipocyte media apparently show twice as much TF activity as the adipocyte media. Anti-TF antibody diminished factor Xa generation, confirming that indeed TF activity is released into the medium. Similar to cell-bound TF, annexin A5 partially inhibited factor $X$ activation. 


\section{TF-driven thrombin generation on preadipocyte and adipocyte cell surfaces}

As a second proof for functional TF, we next investigated the potency of cell-bound TF to generate thrombin in a plasma environment. Therefore, 4 replicate experiments with preadipocytes and adipocytes were performed (Figure 3). Parameters that describe the thrombin generation curves are lag phase: time needed to generate 2-3 nM thrombin; maxR: maximal rate of thrombin generation; thrombin peak height, and time to thrombin peak (Table 1). It is seen that adipocytes are more thrombogenic than preadipocytes because of a statistically significant higher thrombin peak value and maxR. It is apparent that when compared with preadipocytes the higher thrombin production is in accordance with a higher cell surface TF activity of adipocytes. Anti-TF antibodies caused a marked reduction in plasma thrombin generation by both preadipocytes and adipocytes, demonstrating that thrombin generation is indeed driven by TF. In contrast to TF-driven factor Xa generation (see above), the thrombin generation in plasma is completely blocked by annexin A5 incubation. We note therefore that the assembly of prothrombinase, the membrane-bound enzyme complex that coverts prothrombin into thrombin, critically depends on the presence of anionic phospholipids [29].
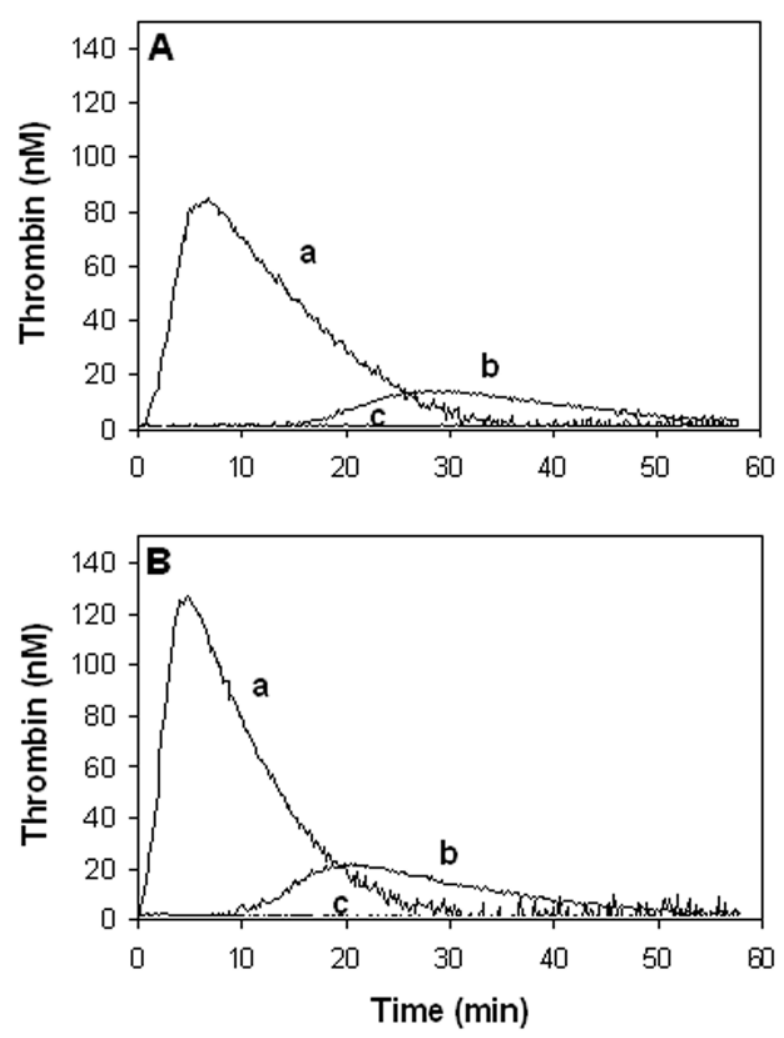

Figure 3: Thrombin generation at cell surfaces. A: preadipocytes and $\mathrm{B}$ : adipocytes grown in 48-well plate were incubated with PFP, CTI $(50 \mu \mathrm{g} / \mathrm{mL})$ and Z-GGR-AMC $(0.4 \mathrm{mM})$. The reaction was started by the addition of $\mathrm{CaCl}_{2}(17 \mathrm{mM})$. Thrombin generation curves were calculated from the fluorescence tracings as described under Methods. a: thrombin generation on untreated cells; b: thrombin generation in the presence of anti-TF $(20 \mu \mathrm{g} / \mathrm{mL})$; c: thrombin generation in the presence of annexin A5 $(0.5 \mu \mathrm{M})$. The curves are the means of 4 experiments. All experiments were performed at $37^{\circ} \mathrm{C}$. 
Table 1: TF-driven thrombin generation in plasma exposed to preadipocytes and mature adipocytes.

\begin{tabular}{lllll}
\hline & Lag time $(\mathrm{min})$ & MaxR $(\mathrm{nM} / \mathrm{min})$ & Peak $(\mathrm{nM})$ & Time-to-peak (min) \\
\hline Preadipocytes & $1.7 \pm 0.1$ & $31 \pm 1$ & $101 \pm 5$ & $7.0 \pm 0.3$ \\
Adipocytes & $1.1 \pm 0.2$ & $47 \pm 5$ & $125 \pm 11$ & $5.7 \pm 0.8$ \\
Preadipocytes + anti-TF & 19.8 & 1.4 & 23 & 34 \\
Adipocytes + anti-TF & 12.8 & 3.0 & 30 & 22 \\
Preadipocytes + AnxA5 & $>60$ & 0 & 0 & $>60$ \\
Adipocytes + AnxA5 & $>60$ & 0 & 0 & $>60$ \\
\hline Table 1: Parameters that describe the thrombin generation curves are lag phase: time needed to generate 2-3 nM \\
thrombin; maxR: maximal rate of thrombin generation; thrombin peak height, and time to thrombin peak.
\end{tabular}

The same thrombin generation experiments were also performed with conditioned medium. In accordance with the lower TF activity in adipocyte-conditioned medium compared to preadipocyte-conditioned medium, also a lower capacity for thrombin generation was found. Anti-TF antibody greatly reduced maxR values and thrombin peak values determined in conditioned medium of preadipocytes and adipocytes (Table 2). Incubation with annexin A5 completely blocked thrombin generation in both conditioned medium of pre-adipocytes and adipocytes.

Table 2: TF-driven thrombin generation in plasma containing conditioned medium

\begin{tabular}{lllll}
\hline & Lag time $(\mathrm{min})$ & $\operatorname{maxR}(\mathrm{nM} / \mathrm{min})$ & Peak $(\mathrm{nM})$ & Time-to-peak (min) \\
\hline Conditioned preadipocyte medium & $3.5 \pm 0.5$ & $10.7 \pm 0.7$ & $43 \pm 1$ & $10.8 \pm 0.5$ \\
Conditioned adipocyte medium & $15 \pm 2$ & $1.4 \pm 0.1$ & $17.0 \pm 0.4$ & $34 \pm 3$ \\
Preadipocytes + anti-TF & $25 \pm 1$ & $0.3 \pm 0.1$ & $8 \pm 1$ & $53 \pm 2$ \\
Adipocytes + anti-TF & $>60$ & 0 & 0 & $>60$ \\
Preadipocytes + AnxA5 & $>60$ & 0 & 0 & $>60$ \\
Adipocytes + AnxA5 & $>60$ & 0 & 0 & $>60$ \\
\hline Table 2: Anti-TF antibody maxR & values and thrombin peak values determined in conditioned medium of \\
preadipocytes and adipocytes.
\end{tabular}

\section{Regulation of TF activity on SGBS cells}

Li et al. [12] and Mobarrez et al. [30] have described statins as a potential treatment strategy of TF-associated health complications due to reduced TF expression. To examine these findings with human adipocytes, preadipocytes and adipocytes were incubated with simvastatin for 24 hours and only functional TF activity assays were performed. TF activity was down regulated by simvastatin. For preadipocytes the maximal reduction was $50 \%$ and for adipocytes $30 \%$ (Figure $4 A$ ). This difference is statistically significant $(p<0.05)$. When preadipocytes and adipocytes were treated with a fixed concentration of simvastatin $(1 \mu \mathrm{M})$ and varying concentrations of mevalonate $(0-100 \mu \mathrm{M})$, TF activity dose dependently increased until the simvastatin effect was almost completely reversed (Figure 4B).

Simvastatin also caused a $53 \%$ decrease in the amount of TF activity in medium exposed to preadipocytes, but did not reduce the shedding of TF activity by adipocytes. As expected, mevalonate $(10 \mu \mathrm{M})$ reversed the inhibitory effect of simvastatin in case of medium exposed to preadipocytes (data not shown). 
A

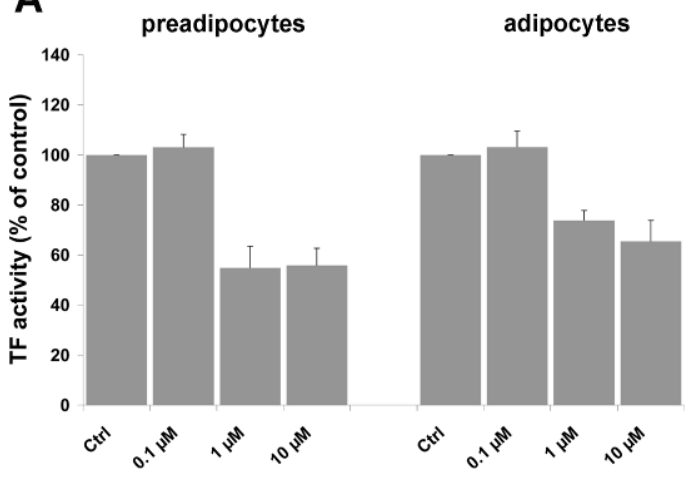

B

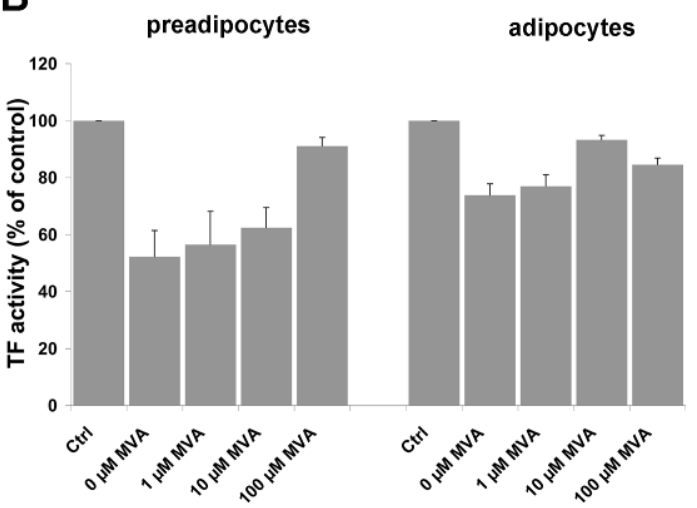

Figure 4: Inhibitory effect of simvastatin on expression of TF activity and the reversion by mevalonate. A: medium of preadipocytes and adipocyte cultures were refreshed with medium containing (0-10 $\mu \mathrm{M})$ simvastatin and cells incubated for 24 hours at $37^{\circ} \mathrm{C}$. TF activity was measured as described under Methods. B: mevalonate-depended reversion of simvastatin-induced down regulation of TF activity was investigated by treatment of preadipocytes and adipocytes for 24 hours with a fixed concentration of simvastatin $(1 \mu \mathrm{M})$ and varying concentrations of mevalonate $(0-100 \mu \mathrm{M})$. All experiments were performed at $37^{\circ} \mathrm{C}$.

\section{Discussion}

Excessive growth of adipose tissue during overweight and obesity is associated with differential expression and secretion of proteins including cytokines, hormones and extracellular matrix proteins [6, 31]. Furthermore, excessive growth of adipose tissue leads to a hypoxic state of the adipocytes which is associated with changes of their protein expression profiles [7] that may lead to stimulation of vascularization and angiogenesis [14, 32]. Increased TF expression in adipose tissue is mostly associated with a hypercoagulable state $[9,23,33]$ while in excessively growing tumor tissue TF expression is primarily related to neo-vascularization [8, 14]. So far, studies of TF expression in adipose tissue have only been done with rodent material and TF expression in relation to human adipocyte differentiation has not been published yet. Therefore, we performed a series of experiments in which human cultured SGBS cells were examined for their capability to express tissue factor. We found, that both human SGBS preadipocytes and adipocytes express functional TF.

The Western blotting analysis detected expression of 70-75 kDa TF bands in human SGBS (pre)adipocytes which is considerably higher than the theoretical molecular masses. A second TF antibody confirmed the observed 70-75 $\mathrm{kDa}$ bands in human SGBS (pre)adipocytes, while the expected $47 \mathrm{kDa}$ band was mainly observed in mouse cells. A possible explanation for the observed $70-75 \mathrm{kDa}$ bands can be that human (pre)adipocyte TF is subjected to additional post-translational modifications. Alternatively, TF from human (pre)adipocytes may form a very stable complex with its natural binding partner factor VII with a molecular mass of $50 \mathrm{kDa}$ [34]. Western blotting shows the relative quantities of a protein but 
does not provide conclusive information about the activity of the detected protein in relation to its expression level. Therefore, TF activity was measured by two different assays.

Based on the cell number, adipocytes express an 8-fold higher TF activity compared to preadipocytes which is in contrast to the expression levels we found with the Western blot analysis. One possible explanation of a higher TF activity is a higher density of anionic phospholipids in the exoleaflet of adipocytes. It has been demonstrated that cell-located TF activity is partially dependent on the presence of externalized PS, albeit this is still a matter of considerable debate $[35,36]$. We found that a saturating concentration of annexin A5 partially (about 65\%) inhibited TF activity, indicating the presence of a PS-dependent and independent TF activity at the surfaces of preadipocytes and mature adipocytes. Another explanation is the increased cell surface size of adipocytes compared to preadipocytes cell surface size [37]. An increased cell surface size can lead to a higher amount of expressed active TF on the cell surface.

An interesting finding was a measurable amount of factor $X$ activation by TF in the medium of both preadipocytes and adipocytes. We observed an elevated level of active TF in preadipocytes medium compared to adipocyte medium, consistent with the Western blot results. Two different isoforms of TF were described by Bogdanov et al. [20]. The first isoform is known as the cell-bound TF (aa 1 - 295), whereas the second isoform lacks the transmembrane domain (aa 252 - 274) and is described as the soluble/secreted alternatively spliced or non-cell-bound TF (asTF). The same group has demonstrated that both isoforms exhibit pro-coagulant activities when they are exposed to phospholipids [20]. More recently, it was reported that there are several asTFs which are differently expressed [19]. In addition, Censarek et al. [21] have demonstrated that asTF has no factor $X$ and thrombin generation activity. This may explain the low TF activity in adipocyte medium compared to a higher cellbound activity as well as the high TF protein expression levels in preadipocyte compared to its relatively low cell bound TF activity.

TF is primarily known for its function in the initiation of the coagulation cascade. Therefore, its function within adipose tissue is mostly associated with the development of a hypercoagulable state which may contribute to the obesity-associated cardiovascular complications [11]. However, TF under hypoxic conditions within fast growing tissues including cancers as well as adipose tissue can contribute to angiogenic processes [38]. The active factor $\mathrm{Xa}$ is known to initiate coagulation but can also trigger angiogenesis by cleavage of PAR-2 [14]. This signaling pathway induces pro-angiogenic factors such as VEGF [17] which is an adipose tissue associated angiogenic factor that promotes vascular endothelial cell proliferation and new vessel formation [39]. Hypoxic conditions in fast growing adipose tissue also induce pro-angiogenic factors [40] although the angiogenesis inducing signaling pathway through hypoxia is still unclear. Hypoxia-inducible factor-1 alpha 
(HIF-1 $\alpha$ ) is stabilized under hypoxia conditions and within tumors its major effect is linked to induction of pro-angiogenic factors [41]. In contrast, Halberg et al. [42] have shown that in fast growing adipose tissue pro-angiogenic factors such VEGF are not affected or induced by HIF-1 1 . Shrivastava et al. [43] described that hypoxia induces angiogenic processes via a TF gene promoter region. One of the promoter regions is called serum response element (SRE) and contains three overlapping Sp1/Erg-1 binding sites which mediates the responses to serum, stress, lipopolysaccaride (LPS) and hypoxia. In addition, Signaevsky and Boing et al. $[22,44]$ showed that asTF stimulated angiogenesis in tumor cells which is promoted through the binding of integrins to the extracellular domain of TF [45]. The alternatively spliced/secreted form is mentioned to promote the process of angiogenesis instead of the coagulation cascade by activating factor $X[22]$.

Besides the TF-dependent factor Xa generation, we also determined the capacity of preadipocytes and adipocytes to enhance TF-dependent thrombin generation in the physiologically more relevant plasma environment. It was found that when cultured preadipocytes were exposed to decalcified plasma, a rapid onset of thrombin generation (within 2 minute) occurred until a thrombin peak height of about $100 \mathrm{nM}$. In good agreement with the higher TF activity, adipocytes have a significantly higher thrombin generating capacity. Indeed, increased thrombin generation and fibrin generation (data not shown) support the hypothesis that TF promotes the hypercoagulated state in obese subjects. However, Belting et al. [14] have considered thrombin as the physical activator of PARs in tumor biology and angiogenesis. PAR-2, which is not cleaved by thrombin, is associated to TF-dependent angiogenesis [14]. In addition, generated thrombin is involved in early inflammation such as acute humoral rejection or sepsis [43]. It can activate PAR-1 and the PAR-1 signaling is an important factor in the evolution of lung inflammation and fibrosis [46]. With our experimental set up sufficient amounts of thrombin were generated which resulted in extravascular fibrin formation. TF-induced thrombin generation and accumulation of fibrin networks are also described as indirect regulation of angiogenesis [47]. Such fibrin network can be seen as a temporary pro-angiogenic matrix that assists the blood vessel infiltration.

Down regulation of TF expression could be interesting to treat TF-induced health problems including hypercoagulation [12], atherothrombotic disease [30], obesity-induced inflammation and in turn insulin resistance [16]. HMG-CoA reductase inhibitors (statins) reduce TF expression in several cell types [48] and also down-regulated TF mRNA and protein expression in cultured rabbit adipocytes [12]. We found that treatment of human (pre)adipocytes with simvastatin in a pharmacologically relevant dose reduced TF activity with about $50 \%$. The inhibitory mechanism likely involves the inhibition of mevalonate synthesis, because addition of mevalonate reversed the inhibitory effect of simvastatin. It is thus feasible that simvastatin prevents the isoprenylation of proteins (small G-proteins) [49] 
which in turn may have profound effects on cellular regulatory pathways including expression of functional TF at the surface of (pre)adipocytes. Moreover, simvastatin and other statins prevent isoprenylation of nuclear lamins. Notably, it has been reported that accumulation of prelamin A causes inhibition of adipogenesis [50]. On the other hand, treatment of obesityassociated diseases via inhibition of TF signaling and in turn inhibition of angiogenesis and vascularization may be responsible for negative systemic effects associated with dysfunction of white adipose tissue (WAT) such as an increased level of inflammation and fibrosis [32]. In conclusion, these data provide evidence for a constitutively active TF expression by human preadipocytes and mature adipocytes. TF signaling may lead to increased vascularization and angiogenesis processes which improve the functioning of WAT. In addition, we hypothesize that in view of the pleiotropy of factor $\mathrm{Xa}$ and thrombin, the here reported generation of these proteases at the surfaces of preadipocytes and adipocytes could also play a pathogenic role in reinforcing thrombosis and inflammation in obese subjects.

\section{References}

1. Gregoire, F.M., Adipocyte differentiation: from fibroblast to endocrine cell. Exp Biol Med (Maywood), 2001. 226(11): p. 997-1002.

2. Deng, Y. and P.E. Scherer, Adipokines as novel biomarkers and regulators of the metabolic syndrome. Ann N Y Acad Sci, 2010. 1212: p. E1-E19.

3. Dimitriadis, G., et al., Impaired postprandial blood flow in adipose tissue may be an early marker of insulin resistance in type 2 diabetes. Diabetes Care, 2007. 30(12): p. 3128-30.

4. Fleischmann, E., et al., Tissue oxygenation in obese and non-obese patients during laparoscopy. Obes Surg, 2005. 15(6): p. 813-9.

5. Stuart Wood, I., et al., Cellular hypoxia and adipose tissue dysfunction in obesity. Proc Nutr Soc, 2009. 68(4): p. 370-7.

6. Goossens, G.H., The role of adipose tissue dysfunction in the pathogenesis of obesity-related insulin resistance. Physiol Behav, 2008. 94(2): p. 206-18.

7. Trayhurn, P., B. Wang, and I.S. Wood, Hypoxia in adipose tissue: a basis for the dysregulation of tissue function in obesity? Br J Nutr, 2008. 100(2): p. 227-35.

8. Zhang, Y., et al., Tissue factor controls the balance of angiogenic and antiangiogenic properties of tumor cells in mice. J Clin Invest, 1994. 94(3): p. 1320-7.

9. Camerer, E., A.B. Kolsto, and H. Prydz, Cell biology of tissue factor, the principal initiator of blood coagulation. Thromb Res, 1996. 81(1): p. 1-41. 
10. Zhao, S.P., et al., Fenofibrate inhibits thrombogenic and fibrinolytic factors expression in adipose tissue of atherosclerotic rabbits. Clin Chim Acta, 2004. 349(1-2): p. 81-6.

11. Samad, F., M. Pandey, and D.J. Loskutoff, Tissue factor gene expression in the adipose tissues of obese mice. Proc Natl Acad Sci U S A, 1998. 95(13): p. 7591-6.

12. Li, J.Q., et al., Atorvastatin reduces tissue factor expression in adipose tissue of atherosclerotic rabbits. Int J Cardiol, 2007. 115(2): p. 229-34.

13. Paborsky, L.R. and R.J. Harris, Post-translational modifications of recombinant human tissue factor. Thromb Res, 1990. 60(5): p. 367-76.

14. Belting, M., J. Ahamed, and W. Ruf, Signaling of the tissue factor coagulation pathway in angiogenesis and cancer. Arterioscler Thromb Vasc Biol, 2005. 25(8): p. 1545-50.

15. Riewald, M. and W. Ruf, Science review: role of coagulation protease cascades in sepsis. Crit Care, 2003. 7(2): p. 123-9.

16. Badeanlou, L., et al., Tissue factor-protease-activated receptor 2 signaling promotes diet-induced obesity and adipose inflammation. Nat Med, 2011. 17(11): p. 1490-7.

17. Liu, Y. and B.M. Mueller, Protease-activated receptor-2 regulates vascular endothelial growth factor expression in MDA-MB-231 cells via MAPK pathways. Biochem Biophys Res Commun, 2006. 344(4): p. 1263-70.

18. Ruf, W., N. Yokota, and F. Schaffner, Tissue factor in cancer progression and angiogenesis. Thromb Res, 2010. 125 Suppl 2: p. S36-8.

19. Chand, H.S., S.A. Ness, and W. Kisiel, Identification of a novel human tissue factor splice variant that is upregulated in tumor cells. Int J Cancer, 2006. 118(7): p. 171320.

20. Bogdanov, V.Y., et al., Alternatively spliced human tissue factor: a circulating, soluble, thrombogenic protein. Nat Med, 2003. 9(4): p. 458-62.

21. Censarek, P., et al., Alternatively spliced human tissue factor (asHTF) is not procoagulant. Thromb Haemost, 2007. 97(1): p. 11-4.

22. Signaevsky, M., et al., Role of alternatively spliced tissue factor in pancreatic cancer growth and angiogenesis. Semin Thromb Hemost, 2008. 34(2): p. 161-9.

23. Samad, F., M. Pandey, and D.J. Loskutoff, Regulation of tissue factor gene expression in obesity. Blood, 2001. 98(12): p. 3353-8.

24. Fischer-Posovszky, P., et al., Human SGBS Cells - a Unique Tool for Studies of Human Fat Cell Biology. Obes Facts, 2008. 1(4): p. 184-189.

25. Wabitsch, M., et al., Characterization of a human preadipocyte cell strain with high capacity for adipose differentiation. Int J Obes Relat Metab Disord, 2001. 25(1): p. 815. 
26. Hemker, H.C., et al., Calibrated automated thrombin generation measurement in clotting plasma. Pathophysiol Haemost Thromb, 2003. 33(1): p. 4-15.

27. Kaneko, T., et al., Induction of Tissue Factor Expression in Endothelial Cells by Basic Fibroblast Growth Factor and its Modulation by Fenofibric acid. Thromb J, 2003. 1(1): p. 6.

28. Wielders, S.J., et al., Factor Xa-driven thrombin generation in plasma: dependency on the aminophospholipid density of membranes and inhibition by phospholipidbinding proteins. Thromb Haemost, 2007. 98(5): p. 1056-62.

29. Heemskerk, J.W., E.M. Bevers, and T. Lindhout, Platelet activation and blood coagulation. Thromb Haemost, 2002. 88(2): p. 186-93.

30. Mobarrez, F., et al., Atorvastatin reduces thrombin generation and expression of tissue factor, P-selectin and GPIIla on platelet-derived microparticles in patients with peripheral arterial occlusive disease. Thromb Haemost, 2011. 106(2): p. 344-52.

31. Wang, P., et al., The secretory function of adipocytes in the physiology of white adipose tissue. J Cell Physiol, 2008. 216(1): p. 3-13.

32. Rutkowski, J.M., K.E. Davis, and P.E. Scherer, Mechanisms of obesity and related pathologies: the macro- and microcirculation of adipose tissue. Febs J, 2009. 276(20): p. 5738-46.

33. Christiaens, V. and H.R. Lijnen, Angiogenesis and development of adipose tissue. Mol Cell Endocrinol, 2010. 318(1-2): p. 2-9.

34. Osterlund, M., et al., Transition state analysis of the complex between coagulation factor VIla and tissue factor: suggesting a sequential domain-binding pathway. Biochem Biophys Res Commun, 2005. 327(3): p. 789-93.

35. Bach, R.R., Tissue factor encryption. Arterioscler Thromb Vasc Biol, 2006. 26(3): p. 456-61.

36. Shaw, A.W., et al., The local phospholipid environment modulates the activation of blood clotting. J Biol Chem, 2007. 282(9): p. 6556-63.

37. Skurk, T., et al., Relationship between adipocyte size and adipokine expression and secretion. J Clin Endocrinol Metab, 2007. 92(3): p. 1023-33.

38. Bluff, J.E., et al., Tissue factor, angiogenesis and tumour progression. Breast Cancer Res, 2008. 10(2): p. 204.

39. Vona-Davis, L. and D.P. Rose, Angiogenesis, adipokines and breast cancer. Cytokine Growth Factor Rev, 2009. 20(3): p. 193-201.

40. Hajer, G.R., T.W. van Haeften, and F.L. Visseren, Adipose tissue dysfunction in obesity, diabetes, and vascular diseases. Eur Heart J, 2008. 29(24): p. 2959-71. 


\section{CHAPTER 4}

41. Oladipupo, S., et al., VEGF is essential for hypoxia-inducible factor-mediated neovascularization but dispensable for endothelial sprouting. Proc Natl Acad Sci U S A, 2011. 108(32): p. 13264-9.

42. Halberg, N., et al., Hypoxia-inducible factor 1alpha induces fibrosis and insulin resistance in white adipose tissue. Mol Cell Biol, 2009. 29(16): p. 4467-83.

43. Shrivastava, S., J.H. McVey, and A. Dorling, The interface between coagulation and immunity. Am J Transplant, 2007. 7(3): p. 499-506.

44. Boing, A.N., et al., Human alternatively spliced tissue factor is not secreted and does not trigger coagulation. J Thromb Haemost, 2009. 7(8): p. 1423-6.

45. van den Berg, Y.W., et al., Alternatively spliced tissue factor induces angiogenesis through integrin ligation. Proc Natl Acad Sci U S A, 2009. 106(46): p. 19497-502.

46. Howell, D.C., et al., Absence of proteinase-activated receptor-1 signaling affords protection from bleomycin-induced lung inflammation and fibrosis. Am J Pathol, 2005. 166(5): p. 1353-65.

47. Rak, J., et al., Tissue factor in cancer and angiogenesis: the molecular link between genetic tumor progression, tumor neovascularization, and cancer coagulopathy. Semin Thromb Hemost, 2006. 32(1): p. 54-70.

48. Brandes, R.P., et al., Withdrawal of cerivastatin induces monocyte chemoattractant protein 1 and tissue factor expression in cultured vascular smooth muscle cells. Arterioscler Thromb Vasc Biol, 2003. 23(10): p. 1794-800.

49. Wang, C.Y., P.Y. Liu, and J.K. Liao, Pleiotropic effects of statin therapy: molecular mechanisms and clinical results. Trends Mol Med, 2008. 14(1): p. 37-44.

50. Rivas, D., R. Akter, and G. Duque, Inhibition of Protein Farnesylation Arrests Adipogenesis and Affects PPARgamma Expression and Activation in Differentiating Mesenchymal Stem Cells. PPAR Res, 2007. 2007: p. 81654. 


\section{Chapter 5}

\section{Hypoxia-mimetic effects in the secretome of human preadipocytes and adipocytes}

Anja Rosenow, Jean-Paul Noben, Freek G. Bouwman, Edwin C.M. Mariman, Johan Renes

BBA - Proteins and Proteomics. 2013; 12 2761-2771 


\begin{abstract}
White adipose tissue (WAT) regulates energy metabolism by secretion of proteins with endocrine and paracrine effects. Dysregulation of the secretome of obesity-associated enlarged WAT may lead to obesity-related disorders. This can be caused by hypoxia as a result of poorly vascularized WAT. The effect of hypoxia on the secretome of human (pre)adipocytes is largely unknown. Therefore, we investigated the effect of $\mathrm{CoCl}_{2}$, a hypoxia mimetic, on the secretome of human SGBS (pre)adipocytes by a proteomics approach combined with bioinformatic analysis. In addition, regulation of protein secretion was examined by protein turnover experiments. As such, secretome changes were particularly associated with protein down-regulation and extracellular matrix protein dysregulation. The observed up-regulation of collagens in adipocytes may be essential for cell survival while down-regulation of collagens in preadipocytes may indicate a disturbed differentiation process. These $\mathrm{CoCl}_{2}$-induced changes reflect WAT dysfunction that ultimately may lead to obesity-associated complications. In addition, 9 novel adipocyte secreted proteins were identified from which 6 were regulated by $\mathrm{CoCl}_{2}$. Mass spectrometry data have been deposited to the ProteomeXchange with identifier PXD000162.
\end{abstract}




\section{Introduction}

Obesity is characterized by expanded white adipose tissue (WAT) [1]. WAT regulates energy metabolism by fatty acid storage and release and by secretion of proteins with endocrine and paracrine effects. A dysregulation of the WAT secretome may lead to obesity-related disorders [2].

Obesity-associated expanded WAT has a decreased capillary density, which leads to $\mathrm{O}_{2}$ deficiency [3] This is related to WAT hypoxia in obese subjects [3] as first postulated by Trayhurn and Wood [4]. Compared to lean persons, obese subjects show an equal blood flow to their WAT despite the enlarged tissue mass [5]. Furthermore, the blood flow to WAT in obese subjects is not increased postprandially [6]. Additionally, the enlarged diameter of hypertrophic adipocytes in obese subjects exceeds the normal $\mathrm{O}_{2}$ diffusion distance of $100-$ $200 \mu \mathrm{m}$ within the tissue [7, 8]. To survive hypoxia, cells switch from oxidative phosphorylation to an anaerobic energy metabolism [9], which influences lipid accumulation [10] and the preadipocyte differentiation capacity [11]. Hypoxia leads to inflammation and cellular dysfunction and as such may lead to increased WAT dysfunction and insulin resistance [12, 13]. In addition, Famulla et. al [14] demonstrated that low oxygen tension (5$10 \%$ ) resulted in smaller lipid droplet size in human adipocytes together with an altered secretion of adiponectin, IL-6, leptin and dipeptidyl eptidase 4. However, The effect of hypoxia on the human (pre)adipocyte secretome is barely understood. Therefore, we investigated the effect of $\mathrm{CoCl}_{2}$, an established hypoxia-mimetic [15], on the secretome of human subcutaneous Simpson-Golabi-Behmel syndrome (SGBS) (pre)adipocytes. Hypoxic responses are mediated via inhibition of prolyl 4-hydroxylases [16] which leads to stabilization of hypoxia inducible factor (HIF-1) [17]. HIF-1 is composed of two subunits, the constitutively expressed HIF-1 $\beta$, and HIF-1 $\alpha$ which is stabilized upon hypoxic conditions [17]. HIF-1 $\alpha$ serves as a hypoxia-induced transcription factor that targets genes involved in development, metabolism, inflammation and integrative physiology [18, 19]. SGBS cells are ideal fat cells to culture [20] and to investigate the (pre)adipocyte secretome [21, 22]. Here, secretome profiling was performed by two-dimensional gel electrophoresis (2DE) and liquid chromatography-electrospray ionization tandem mass spectrometry (LC-ESI MS/MS). In addition, protein turnover, by incorporation of stable isotope labeled phenylalanine, was examined to analyze the effect of $\mathrm{CoCl}_{2}$ on protein synthesis. This $\mathrm{CoCl}_{2}$ effect might represent an underlying mechanism for hypoxia-induced dysregulation of the (pre)adipocyte secretome. 


\section{Methods}

\section{Materials}

Culture media, $0.5 \%$ trypsin-EDTA and 10,000 u/ml Penicillin/Streptomycin were obtained from Life Technologies (Bleiswijk, The Netherlands). Fetal bovine serum (FBS) was purchased from Bodinco (Alkmaar, The Netherlands). Additional cell culture supplements, protease inhibitor cocktail, cobalt (II) chloride hexahydrate (CoCl2) phenylmethylsulfonylfluoride (PMSF), DL-dithiothreitol (DTT), 3-[(3-cholamidopropyl) dimethyl-amonio]-1-propanesulfonate (CHAPS), a-cyano-4-hydroxyl-cinnamic acid (CHCA), trifluoroacetic acid (TFA) and acetonitrile (ACN) were obtained from Sigma-Aldrich (Zwijndrecht, The Netherlands). Immobilized pH gradient (IPG) buffer (pH 3-11, nonlinear), nitrocellulose membrane, Dry-Strip cover fluid and immobiline Dry-Strip ( $\mathrm{pH} 3-11$, nonlinear, $24 \mathrm{~cm}$ ) were from GE Healthcare (Diegem, Belgium). L-(ring-2,3,4,5,6 2H5) phenylalanine was purchased from Cambridge Isotope Laboratories (Andover, MA, USA)

\section{Cell culture}

Human Simpson-Golabi-Behmel syndrome (SGBS) cells were obtained from Prof. Dr. M. Wabitsch (University of Ulm, Germany) [20] and cultured as described [21]. 90\% confluent preadipocytes were differentiated into mature adipocytes for 13 days as previously reported [21]. On average $77 \%$ of the preadipocytes differentiated into mature adipocytes. To determine cell numbers, adipocytes were counted with a raster ocular. Preadipocytes were trypsinized and counted with a hemocytometer. The number of adipocytes per $150 \mathrm{~mm}$ Petri dish was $1.7 \pm 1.3 \times 10^{6}$ (mean $\pm S D, n=5$ ) and the number of preadipocytes per $150 \mathrm{~mm}$ Petri dish was $2.2 \pm 0.36 \times 10^{6}$ (mean $\left.\pm S D, n=5\right)$.

\section{Culture conditions for hypoxia experiments}

To induce a hypoxic state, $90 \%$ confluent preadipocytes or fully differentiated adipocytes were treated with or without (control) $100 \mu \mathrm{M} \mathrm{CoCl} 2$ in serum-free and phenol red-free DMEM/F12 medium containing $66 \mathrm{nM}$ insulin for 48h. A time course experiment was performed with continuous $\mathrm{CoCl}_{2}$ incubation up to $72 \mathrm{~h}$ and with repeated incubation of 100 $\mu \mathrm{M} \mathrm{CoCl}{ }_{2}$ every $24 \mathrm{~h}$ for the $48 \mathrm{~h}$ and $72 \mathrm{~h}$ time points.

\section{Culture conditions protein turnover experiments}

Protein turnover was measured as described by Bouwman et al. [23]. Briefly, $35.48 \mathrm{mg} / \mathrm{L}$ stable isotope $\mathrm{L}$-(ring-2,3,4,5,6 ${ }^{2} \mathrm{H}_{5}$ ) phenylalanine was added to DMEM/F12 medium containing $35.48 \mathrm{mg} / \mathrm{L}$ non-labeled L-phenylalanine to obtain a 1:1 ratio of non-labeled and labeled phenylalanine. This allowed a maximal replacement of non-labeled phenylalanine in proteins up to $50 \%$. For time course experiments cells were washed twice with PBS buffer 
and incubated with phenol red free DMEM/F12 medium containing $66 \mathrm{nM}$ insulin and 35.48 $\mathrm{mg} / \mathrm{L}$ stable isotope $\mathrm{L}$-(ring-2,3,4,5,6 ${ }^{2} \mathrm{H}_{5}$ ) phenylalanine for $0,24,48$ and $72 \mathrm{~h}$.

\section{Determination of HIF-1 $\alpha$ by Western blotting}

Adipocytes were solubilised in lysis buffer (0.5 M Tris (pH 6.8), 10\% SDS, 10\% glycerol, 1 mM PMSF, $0.2 \mathrm{mM}$ DTT, 1 Complete mini $^{\mathrm{TM}}$ tablet (Roche Diagnostics, Almere, The Netherlands) per $10 \mathrm{ml}$ ). $50 \mu \mathrm{g}$ protein was separated by $10 \%$ SDS-PAGE and transferred to a NC membrane. The membrane was blocked with $5 \%$ dry milk powder and probed with primary polyclonal rabbit antibodies against human HIF-1 $\alpha$ (Novus Biologicals, Cambridge, UK) or polyclonal mouse antibodies against $\beta$-actine (Santa Cruz biotechnology, Heidelberg, Germany). As secondary antibodies swine anti-rabbit IgG (DAKO, Glostrup, Denmark) and rabbit anti-mouse IgG (DAKO) were used. Bound antibodies were visualized with chemiluminescence (SuperSignal west Femto reagent, Thermo Scientific, Etten-Leur, The Netherlands).

\section{Sample preparation for 2-DE and protein turnover experiments}

Culture media were collected, supplemented with protease inhibitor cocktail, transferred to dialysis tubes (2-kDa molecular-weight cut-off, Carl Roth $\mathrm{GmbH}$, Karlsruhe, Germany) and dialyzed against $20 \mathrm{mM}$ ammonium bicarbonate at $4^{\circ} \mathrm{C}$ for $48 \mathrm{~h}$ with $10-12 \mathrm{x}$ buffer changes. The dialyzed medium samples were freeze-dried, dissolved in fresh rehydration buffer ( $8 \mathrm{M}$ urea, $2 \% \mathrm{w} / \mathrm{v}$ CHAPS and $65 \mathrm{mM} \mathrm{DTT}$ ) and centrifuged at $20000 \mathrm{xg}$ at $10^{\circ} \mathrm{C}$ for $30 \mathrm{~min}$. The protein concentrations were determined with a Bradford-based protein assay kit (Bio-Rad Laboratories, Veenendaal, The Netherlands) [23]. All samples were stored at $-80^{\circ} \mathrm{C}$.

\section{2-DE}

Protein samples derived from $1.7 \times 10^{6}$ adipocytes and $4.3 \times 10^{6}$ preadipocytes were used for 2-DE analysis according to Bouwman et al. [22] but with different IPG strips (pH 3-11, $24 \mathrm{~cm}$, nonlinear). $100 \mu \mathrm{g}$ of total protein in a volume of $450 \mu$ containing $0.5 \%(\mathrm{v} / \mathrm{v})$ IPG buffer was loaded onto the IPG strips. For protein profiling, 4 independent replicates were made from $\mathrm{CoCl}_{2}$ treated and non-treated adipocytes and 3 independent replicates were made from $\mathrm{CoCl}_{2}$ treated and non-treated preadipocytes (Supplement 1 ). The gels were stained with Flamingo fluorescent gel stain according to the manufacturer's protocol (Bio-Rad Laboratories). To visualize the proteins, gels were scanned by the Molecular Imager FX (BioRad Laboratories). 


\section{CHAPTER 5}

\section{Image analysis}

Stained gels were processed by PDQuest 8.0 (Bio-Rad Laboratories). Data were normalized with respect to the total density of gel image. Each group was formed from samples of independent experiments with the same treatment. Protein spots were regarded as significantly differentially expressed if the average spot intensity between the $\mathrm{CoCl}_{2}$ treated and non-treated groups differed more than 1.5-fold $p<0.05$ (Student's t-test). The criteria for indicating a trend was a spot intensity difference of more than 1.5 -fold and $0.05<p<0.1$. For subsequent protein identification, gels with differentially expressed spots were re-stained with SYPRO Ruby Protein Stain according to the manufacturer's protocol (Bio-Rad Laboratories).

\section{SDS-PAGE for protein turnover experiments}

$20 \mu \mathrm{g}$ protein sample of each condition was separated by $12 \%$ SDS-PAGE. Gels were stained with CBB according to the manufacturer's protocol (Bio-Rad Laboratories). Each lane was cut into 32 similar pieces and subjected to in-gel digestion and MS for protein turnover analysis according to [22].

\section{MS/MS and protein identification}

Excised 2-DE spots and pieces from SDS-PAGE gels were in-gel digested as described by Bouwman et al. [22]. All 2-DE spots were analyzed by LC-ESI MS/MS, as described by Dumont et al. [24]

With respect to protein turnover experiments peptide mass fingerprints were obtained from 1 $\mu \mathrm{l}$ of each peptide mixture and $1 \mu \mathrm{l}$ matrix solution (10 mg/ml CHCA in 50\% ACN/0.1\% TFA). These mixtures were spotted onto an Opti-TOFTM 384 well plate and analyzed with a 4800 MALDI TOF/TOF analyzer (AB Sciex, Foster City, USA). The instrument was operated as described by Cranenburg et.al. [25]. For semi-quantitative labeling measurement, the area of the $M+5$ peaks was divided by that of the $M$ peak $\times 100 \%$ ratio as described by Bouwman et al. [22].

\section{Database search}

With respect to LC-ESI-MS/MS, LCQ Xcalibur v2.0 SR2 raw files and spectra were selected from Proteome Discover1.2 software (Thermo Scientific) with the following settings: minimal peak count 50; total intensity threshold 4000; and $S / N \geq 6$. Peak lists were searched with Sequest v1.2.0.208 and Mascot v2.3.0.1 against EMBL-EBI International Protein Index database for human proteins (version 3.78, 86702 entries). Search engine results were combined and validated by Scaffold v3.00.07 (Proteome Software, Portland, OR, USA) with minimum peptide and protein probability set to $\geq 95 \%$, followed by manual curation considering mass spectral (annotation) quality. To identify the proteins in the protein turnover 
experiments the peptide mass lists generated by MALDI-MS were searched against the SwissProt database. For protein identification one miss-cleavage was tolerated, carbamidomethylation was set as a fixed modification and oxidation of methionine as an optional modification. The peptide mass tolerance was set to $100 \mathrm{ppm}$ and $150 \mathrm{ppm}$. No restrictions were made on the protein $\mathrm{Mr}$ and the $\mathrm{pl}$. A protein was regarded as identified when it had a significant MASCOT probability score $(p<0.05)$ and at least five peptide mass hits or sequence coverage of at least $30 \%$ of the complete protein sequence. The mass spectrometry proteomics data have been deposited to the ProteomeXchange Consortium (http://proteomecentral.proteomexchange.org

<http://proteomecentral.proteomexchange.org>) via the PRIDE partner repository [26] with the dataset identifier PXD000162 and DOI 10.6019/PXD000162." Mascot DAT files were processed by PRIDE Converter software [27] and submitted via ProteomeXchange (http://www.proteomexchange.org) to the PRIDE database [28], project accession number: 28672-28710. MS data can be visualized using PRIDE Inspector [29] (http://tinyurl.com/csffalc) and Scaffold .sf3 free viewer (https://proteomecommons.org/tool.jsp?i=1009).

\section{Validation of secreted protein candidates}

For verification of secreted protein candidates an amino acid sequence analysis was performed with SignalP 3.0 and SecretomeP 2.0 (CBS, Technical University Copenhagen, Denmark, www. cbs.tdu.dk). SignalP 3.0 was used to verify the presence and location of signal peptide cleavage sites in amino acid sequences. SecretomeP 2.0 was applied to categorize proteins as non-classical secreted in case they were devoid of a signal peptide but obtained an NN-score above the threshold of 0.5. In addition, ExoCarta analyses were performed to evaluate how many of the identified proteins are known to be exosomal by introducing the protein gene name to the ExoCarta (NHMRC Biomedical Research Fellow, Bundoora, Australia, www.exocarta.org).

\section{Effect of $\mathrm{CoCl}_{2}$-treatment on lipolysis}

To study the effect of $\mathrm{CoCl}_{2}$ on lipolysis adipocytes were cultured and differentiated as described above. After 13 days of differentiation the medium was removed and adipocytes were washed twice with PBS. Subsequently, adipocytes were cultured with phenol red free DMEM/F12 medium containing $66 \mathrm{nM}$ insulin and $\mathrm{CoCl}_{2}$ as follows. For the $\mathrm{CoCl}_{2}$ concentration study adipocytes were incubated once with $50,75,100$ and $200 \mu \mathrm{M} \mathrm{CoCl}$ for 48h. During the $\mathrm{CoCl}_{2}$ time course study the adipocytes were treated once with $100 \mu \mathrm{M}$ $\mathrm{CoCl}_{2}$ for $4 \mathrm{~h}, 8 \mathrm{~h}, 16 \mathrm{~h}, 24 \mathrm{~h}, 48 \mathrm{~h}$ and $72 \mathrm{~h}$. For $48 \mathrm{~h}$ and $72 \mathrm{~h}$ time points adipocytes were also repeatedly treated with $100 \mu \mathrm{M} \mathrm{CoCl}$ every $24 \mathrm{~h}$. The non-treated cells were cultured with 


\section{CHAPTER 5}

phenol red free DMEM/F12 medium containing 66nM insulin. The effect of different hypoxia conditions on lipolysis was determined by glycerol release and intracellular triglyceride (TG) contents.

\section{Glycerol release measurement}

Glycerol released in the culture medium was measured by a fluorimetric assay using a Cobas-Fara Instrument (Roche Diagnostics, Almere, The Netherlands). The amount of glycerol release was corrected for the number of counted cells.

\section{Measurement of TG content by Oil red O (ORO) staining}

Adipocytes were fixed with $3.7 \%$ formaldehyde for $10 \mathrm{~min}$ at room temperature. Fixed cells were incubated with a filtered ORO solution (1\% ORO in $60 \%$ isopropanol) for $30 \mathrm{~min}$. Finally cells were washed 6 times with $70 \%$ ethanol. Images were taken with a Nikon TE 200 eclipse phase contrast microscope equipped with digital image acquisition. To determine the intracellular TG accumulation, the ORO stain was extracted with DMSO and measured by spectrophotometry at $540 \mathrm{~nm}$. The amount of intracellular ORO content was corrected for the number of cells.

\section{Results}

\section{HIF-1 $\alpha$ protein stabilization by $\mathrm{CoCl}_{2}$ incubation}

Stabilization of HIF-1a indicates hypoxia [31]. In adipocytes HIF-1 $\alpha$ was significantly increased up to $72 \mathrm{~h}$ (Figure 1). Repeated treatment every $24 \mathrm{~h}$ with new $\mathrm{CoCl}_{2}(100 \mu \mathrm{M})$ during $48 \mathrm{~h}$ and $72 \mathrm{~h}$ incubations additionally increased HIF-1 $\alpha$. Maximal HIF-1 $\alpha$ protein levels were already observed after $4 \mathrm{~h}$ incubation; however, this appeared too short for secretome profiling. Consequently, subsequent 2DE and protein turnover experiments were performed with $48 \mathrm{~h} \mathrm{CoCl}_{2}$ incubation, with a repeated treatment after $24 \mathrm{~h}$. Preadipocytes were treated similarly for a valid comparison of the (pre)adipocytes secretome and protein turnover data sets. 

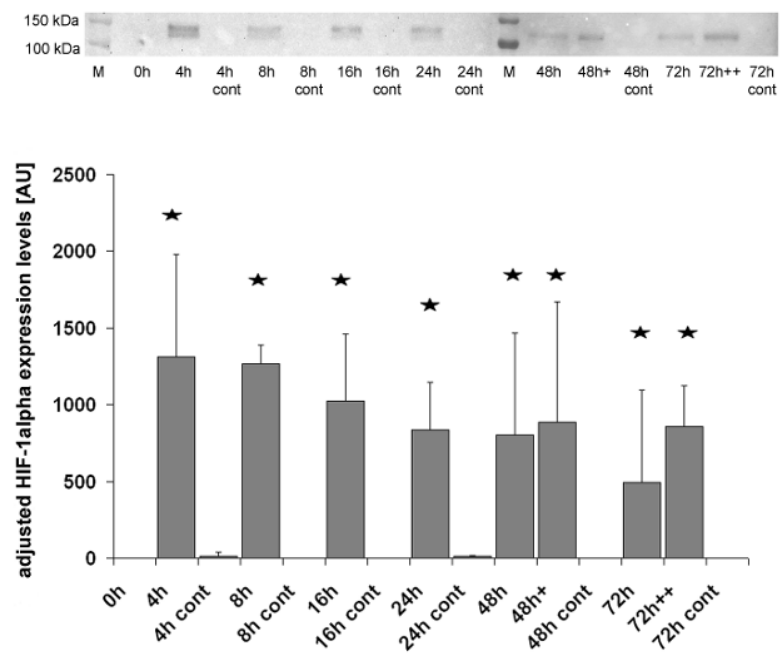

Figure 1: HIF-1a protein expression in $\mathrm{CoCl}_{2}$-treated adipocytes. HIF-1 $\alpha$ protein levels in 13 days differentiated SGBS adipocytes (50 $\mu$ g protein lysate). Cells were continuously incubated up to $72 \mathrm{~h}$ with 100 $\mu \mathrm{M} \mathrm{CoCl}_{2} .48 \mathrm{~h}^{+}$or $72 \mathrm{~h}^{++}$indicate every $24 \mathrm{~h}$ repeated $100 \mu \mathrm{M} \quad \mathrm{CoCl}_{2}$ refreshment. Normalization was done by using the $\beta$-actin protein signal. Reported values are means \pm SD of 3 biological replicates and * indicates statistically significant differences with $p<0.05$ compared to control (cont).

\section{Secretome analysis of $\mathrm{CoCl}_{2}$-treated adipocytes}

LC-ESI-MS/MS analysis, which resulted in 39 identified spots documented in 30 unique spots (Table 1, Figure 2 and Supplement 1). Subsequent data analysis revealed 35 unique proteins which were sub-categorized into classical secreted (17), non-classical secreted (6) and intracellular (12). Presence of intracellular proteins in the secretome can be related to cell death or cell leakage and are presented because of their potential influence on spot regulation. The category "classical secreted" proteins represents proteins which are already known as secreted according to UniProtKB/Swiss-Prot, version 69 or have at least one signal peptide cleavage site according to SignalP v3.0. This group was sub-categorized into extracellular matrix (ECM), processing, regulation/signaling and immune regulation proteins. The largest sub-group comprised ECM proteins, including collagens and fibronectin. Analysis of secretion features by secretomeP v2.0 indicated 6 additional proteins as non-classical secreted proteins. Most of the identified proteins were down-regulated. Serum albumin and full length collagen isoforms were induced by hypoxia (Table 1).

Table 1: Identification of secreted proteins of $\mathrm{CoCl}_{2}$-treated or non-treated (pre)adipocytes

\begin{tabular}{|c|c|c|c|c|c|c|c|c|c|c|}
\hline \multirow{2}{*}{$\begin{array}{l}\text { accession } \\
\text { number }\end{array}$} & \multirow[t]{2}{*}{ protein } & \multirow{2}{*}{$\begin{array}{l}\text { Exo } \\
\text { Carta }\end{array}$} & \multicolumn{4}{|c|}{ adipocytes } & \multicolumn{4}{|c|}{ preadipocytes } \\
\hline & & & ID & $\begin{array}{l}\mathrm{MW} \\
{[\mathrm{kDa}]}\end{array}$ & $\begin{array}{l}\text { Fold } \\
\text { change }\end{array}$ & $p$-value & ID & $\begin{array}{c}\mathrm{MW} \\
{[\mathrm{kDa}]}\end{array}$ & $\begin{array}{c}\text { Fold } \\
\text { change }\end{array}$ & $p$-value \\
\hline \multicolumn{11}{|c|}{$\begin{array}{l}\text { classical secreted } \\
\text { extra cellular matrix }\end{array}$} \\
\hline P27797 & CALR & yes & 1 & 68.9 & $-1.75 E+00$ & 8.37E-02 & & & & \\
\hline \multirow[t]{4}{*}{ P02452 } & COL1A1 & yes & 7 & 164.6 & $1.04 \mathrm{E}+01$ & 2.06E-02 & 3 & $\begin{array}{c}30.4 \\
(\mathrm{~N}- \\
\text { term) }\end{array}$ & $4.3 \mathrm{E}+00$ & $9.06 \mathrm{E}-02$ \\
\hline & & & 9 & 164.2 & $2.79 \mathrm{E}+00$ & 8.08E-02 & 3 & $\begin{array}{c}37.0 \\
(\mathrm{~N}- \\
\text { term })\end{array}$ & $7.3 \mathrm{E}+00$ & 2.64E-02 \\
\hline & & & 15 & 145.8 & $5.88 \mathrm{E}+00$ & 2.84E-02 & 4 & $\begin{array}{c}26.3 \\
(\mathrm{~N}- \\
\text { term) }\end{array}$ & $8.7 \mathrm{E}+00$ & 2.68E-02 \\
\hline & & & 19 & 93.9 & $-5.03 E+00$ & $6.45 \mathrm{E}-02$ & 9 & $\begin{array}{c}80.7 \\
(\mathrm{~N}- \\
\text { term })\end{array}$ & $1.1 \mathrm{E}+01$ & $9.70 \mathrm{E}-02$ \\
\hline
\end{tabular}


Table 1: Identification of secreted proteins of $\mathrm{CoCl}_{2}$-treated or non-treated (pre)adipocytes

\begin{tabular}{|c|c|c|c|c|c|c|c|c|c|c|}
\hline \multirow{2}{*}{$\begin{array}{c}\text { accession } \\
\text { number }\end{array}$} & \multirow[t]{2}{*}{ protein } & \multirow{2}{*}{$\begin{array}{l}\text { Exo } \\
\text { Carta }\end{array}$} & \multicolumn{4}{|c|}{ adipocytes } & \multicolumn{4}{|c|}{ preadipocytes } \\
\hline & & & ID & $\begin{array}{c}\mathrm{MW} \\
{[\mathrm{kDa}]}\end{array}$ & $\begin{array}{l}\text { Fold } \\
\text { change }\end{array}$ & $p$-value & ID & $\begin{array}{c}\mathrm{MW} \\
{[\mathrm{kDa}]}\end{array}$ & $\begin{array}{c}\text { Fold } \\
\text { change }\end{array}$ & $p$-value \\
\hline \multicolumn{11}{|c|}{$\begin{array}{l}\text { classical secreted } \\
\text { extra cellular matrix }\end{array}$} \\
\hline \multirow[t]{42}{*}{ P02452 } & COL1A1 & yes & 7 & 164.6 & $1.04 \mathrm{E}+01$ & 2.06E-02 & 10 & $\begin{array}{c}77.8 \\
(\mathrm{~N}- \\
\text { term) }\end{array}$ & 1.1E+05 & 7.38E-05 \\
\hline & & & & & & & 10 & $\begin{array}{c}36.9 \\
(\mathrm{C}- \\
\text { term) }\end{array}$ & $-2.1 \mathrm{E}+04$ & 3.04E-05 \\
\hline & & & & & & & 10 & $\begin{array}{c}36.6 \\
(\mathrm{C}- \\
\text { term })\end{array}$ & $-3.1 \mathrm{E}+00$ & $7.40 \mathrm{E}-02$ \\
\hline & & & & & & & 10 & 155.4 & $-2.1 \mathrm{E}+01$ & 2.23E-02 \\
\hline & & & & & & & 10 & 155.8 & $-7.2 \mathrm{E}+04$ & $2.55 \mathrm{E}-03$ \\
\hline & & & & & & & 10 & 205.8 & $-6.3 E+00$ & $4.09 \mathrm{E}-02$ \\
\hline & & & & & & & 10 & 258.1 & $-4.0 \mathrm{E}+00$ & $4.63 \mathrm{E}-02$ \\
\hline & & & & & & & 10 & 257.6 & $-1.1 \mathrm{E}+05$ & 4.91E-02 \\
\hline & & & & & & & 10 & 246.6 & $-2.1 \mathrm{E}+05$ & $1.71 \mathrm{E}-04$ \\
\hline & & & & & & & 10 & 259.5 & $-1.1 \mathrm{E}+05$ & 1.44E-05 \\
\hline & & & & & & & 10 & 261.5 & $-2.3 E+05$ & $1.38 \mathrm{E}-04$ \\
\hline & & & & & & & 10 & 245.1 & $-4.5 E+05$ & $4.24 \mathrm{E}-04$ \\
\hline & & & & & & & 10 & $\begin{array}{c}75.6 \\
(\mathrm{C}- \\
\text { term) }\end{array}$ & $-1.7 \mathrm{E}+04$ & 7.01E-04 \\
\hline & & & & & & & 10 & 260.1 & $-3.5 E+04$ & 2.77E-04 \\
\hline & & & & & & & 10 & 260.3 & $-3.3 E+04$ & 7.54E-05 \\
\hline & & & & & & & 10 & 265.5 & $-3.5 E+04$ & $1.75 \mathrm{E}-03$ \\
\hline & & & & & & & 11 & $\begin{array}{c}45.0 \\
(\mathrm{~N}- \\
\text { term) }\end{array}$ & $3.8 \mathrm{E}+00$ & 3.54E-02 \\
\hline & & & & & & & 12 & $\begin{array}{c}94.9 \\
(\mathrm{~N}- \\
\text { term) }\end{array}$ & $1.2 \mathrm{E}+05$ & 3.53E-03 \\
\hline & & & & & & & 13 & $\begin{array}{c}92.0 \\
(\mathrm{~N}- \\
\text { term })\end{array}$ & $9.6 \mathrm{E}+04$ & $1.55 \mathrm{E}-04$ \\
\hline & & & & & & & 19 & $\begin{array}{c}36.5 \\
(\mathrm{C}- \\
\text { term) }\end{array}$ & $-2.3 E+00$ & 7.44E-02 \\
\hline & & & & & & & 19 & $\begin{array}{c}38.9 \\
(\mathrm{C}- \\
\text { term) }\end{array}$ & $-3.9 E+05$ & 1.16E-02 \\
\hline & & & & & & & 21 & 132.3 & $-1.6 \mathrm{E}+00$ & $3.13 \mathrm{E}-02$ \\
\hline & & & & & & & 22 & 132.0 & $-3.0 \mathrm{E}+00$ & 3.57E-02 \\
\hline & & & & & & & 24 & 232.7 & $-5.9 \mathrm{E}+00$ & 5.04E-02 \\
\hline & & & & & & & 24 & 234.8 & $-3.1 \mathrm{E}+00$ & 8.01E-02 \\
\hline & & & & & & & 25 & 235.7 & $-9.5 E+00$ & $2.44 \mathrm{E}-03$ \\
\hline & & & & & & & 25 & 236.1 & $-4.2 \mathrm{E}+00$ & $9.65 \mathrm{E}-02$ \\
\hline & & & & & & & 25 & 236.8 & $-5.1 \mathrm{E}+00$ & $1.53 \mathrm{E}-03$ \\
\hline & & & & & & & 26 & 236.1 & $-4.2 \mathrm{E}+00$ & $2.98 \mathrm{E}-02$ \\
\hline & & & & & & & 27 & 287.2 & $-1.0 \mathrm{E}+05$ & $2.93 \mathrm{E}-02$ \\
\hline & & & & & & & 28 & 250.1 & $-4.1 \mathrm{E}+00$ & $9.63 \mathrm{E}-03$ \\
\hline & & & & & & & 28 & 260.1 & $-4.9 \mathrm{E}+00$ & 2.06E-02 \\
\hline & & & & & & & 36 & 257.4 & $-5.7 E+00$ & $9.56 \mathrm{E}-02$ \\
\hline & & & & & & & 36 & 244.8 & $-7.4 \mathrm{E}+04$ & 8.88E-05 \\
\hline & & & & & & & 36 & 241.1 & $-7.7 \mathrm{E}+04$ & 8.82E-06 \\
\hline & & & & & & & 36 & 239.2 & $-2.4 \mathrm{E}+02$ & 6.77E-02 \\
\hline & & & & & & & 36 & 238.7 & $-2.9 \mathrm{E}+00$ & 4.44E-02 \\
\hline & & & & & & & 36 & 238.3 & $-7.1 \mathrm{E}+03$ & $3.48 \mathrm{E}-02$ \\
\hline & & & & & & & 36 & 237.3 & $-6.3 E+00$ & 7.93E-02 \\
\hline & & & & & & & 36 & 244.2 & $-3.1 \mathrm{E}+04$ & $1.12 \mathrm{E}-03$ \\
\hline & & & & & & & 40 & 242.6 & $-5.4 \mathrm{E}+00$ & 4.02E-02 \\
\hline & & & & & & & 41 & 244.9 & $-3.7 E+01$ & 4.31E-02 \\
\hline \multirow[t]{7}{*}{ P02461 } & COL3A1 & yes & & 141.5 & $-2.83 E+00$ & $6.56 \mathrm{E}-02$ & 24 & 232.7 & $-5.9 \mathrm{E}+00$ & $5.04 \mathrm{E}-02$ \\
\hline & & & 7 & 164.6 & $1.04 \mathrm{E}+01$ & 2.06E-02 & 24 & 234.8 & $-3.1 \mathrm{E}+00$ & $8.01 \mathrm{E}-02$ \\
\hline & & & 9 & 164.2 & $2.79 \mathrm{E}+00$ & 8.08E-02 & 26 & 236.1 & $-4.2 \mathrm{E}+00$ & $2.98 \mathrm{E}-02$ \\
\hline & & & 19 & 93.9 & $-5.03 E+00$ & $6.45 \mathrm{E}-02$ & 28 & 250.1 & $-4.1 \mathrm{E}+00$ & $9.63 \mathrm{E}-03$ \\
\hline & & & & & & & 28 & 260.1 & $-4.9 \mathrm{E}+00$ & $2.06 \mathrm{E}-02$ \\
\hline & & & & & & & 40 & 242.6 & $-5.4 \mathrm{E}+00$ & $4.02 \mathrm{E}-02$ \\
\hline & & & & & & & 41 & 244.9 & $-3.7 \mathrm{E}+01$ & $4.31 \mathrm{E}-02$ \\
\hline P02462 & COL4A1 & yes & & & & & 42 & 264.8 & $-3.2 \mathrm{E}+04$ & 1.14E-04 \\
\hline P20908 & COL5A1 & yes & & & & & 11 & 45.0 & $3.8 \mathrm{E}+00$ & $3.54 \mathrm{E}-02$ \\
\hline
\end{tabular}


Table 1: Identification of secreted proteins of $\mathrm{CoCl}_{2}$-treated or non-treated (pre)adipocytes

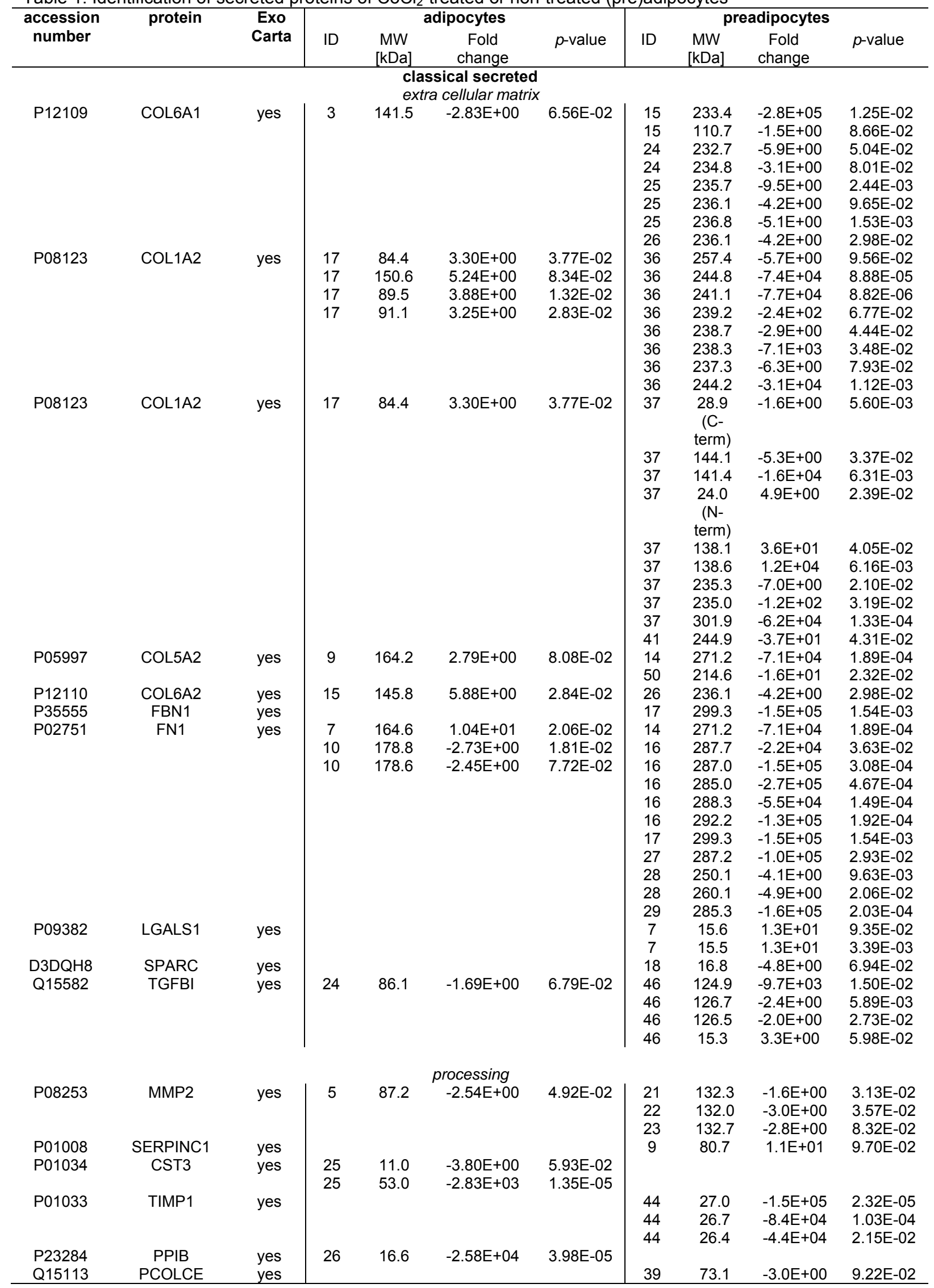


Table 1: Identification of secreted proteins of $\mathrm{CoCl}_{2}$-treated or non-treated (pre)adipocytes

\begin{tabular}{|c|c|c|c|c|c|c|c|c|c|c|}
\hline \multirow{2}{*}{$\begin{array}{c}\text { accession } \\
\text { number }\end{array}$} & \multirow[t]{2}{*}{ protein } & \multirow{2}{*}{$\begin{array}{l}\text { Exo } \\
\text { Carta }\end{array}$} & \multicolumn{4}{|c|}{ adipocytes } & \multicolumn{4}{|c|}{ preadipocytes } \\
\hline & & & ID & $\begin{array}{c}\mathrm{MW} \\
{[\mathrm{kDa}]}\end{array}$ & $\begin{array}{c}\text { Fold } \\
\text { change }\end{array}$ & $p$-value & ID & $\begin{array}{c}\mathrm{MW} \\
{[\mathrm{kDa}]}\end{array}$ & $\begin{array}{c}\text { Fold } \\
\text { change }\end{array}$ & $p$-value \\
\hline \multicolumn{11}{|c|}{$\begin{array}{l}\text { classical secreted } \\
\text { regulation/signaling }\end{array}$} \\
\hline \multirow[t]{3}{*}{ B7Z8Q2 } & $\begin{array}{c}\text { cDNA } \\
\text { FLJ55606* }\end{array}$ & NR & & & & & 5 & 101.0 & $1.6 \mathrm{E}+00$ & $3.29 \mathrm{E}-02$ \\
\hline & & & & & & & 6 & 118.0 & $2.3 \mathrm{E}+00$ & 5.47E-02 \\
\hline & & & & & & & 12 & 94.9 & $1.2 \mathrm{E}+05$ & $3.53 \mathrm{E}-03$ \\
\hline \multirow[t]{2}{*}{ Q9BRK5 } & SDF4 & yes & & & & & 5 & 101.0 & $1.6 \mathrm{E}+00$ & $3.29 \mathrm{E}-02$ \\
\hline & & & & & & & 9 & 80.71 & $1.1 \mathrm{E}+01$ & $9.70 \mathrm{E}-02$ \\
\hline P11021 & HSPA5 & yes & & & & & 22 & 132.0 & $-3.0 \mathrm{E}+00$ & 3.57E-02 \\
\hline \multirow[t]{2}{*}{ P02649 } & APOE & yes & 4 & 39.9 & $-5.05 E+00$ & 3.10E-02 & & & & \\
\hline & & & 14 & 35.4 & $-6.62 E+03$ & 3.96E-02 & & & & \\
\hline Q9Y240 & CLEC11A & yes & & & & & 4 & 26.3 & $8.7 E+00$ & 2.68E-02 \\
\hline \multirow[t]{2}{*}{043852} & CALU* $^{*}$ & yes & & & & & 3 & 30.4 & $4.3 E+00$ & 9.06E-02 \\
\hline & & & & & & & 3 & 37.0 & $7.3 E+00$ & 2.64E-02 \\
\hline P36222 & CHI3L1 & NR & 23 & 42.8 & $-2.62 E+00$ & 9.01E-02 & & & & \\
\hline P14625 & HSP90B1 & yes & & & & & 13 & 92.0 & $9.6 \mathrm{E}+04$ & $1.55 \mathrm{E}-04$ \\
\hline Q12841 & FSTL1 & NR & & & & & 9 & 80.7 & $1.1 \mathrm{E}+01$ & $9.70 \mathrm{E}-02$ \\
\hline 014498 & ISLR & NR & & & & & 5 & 101.0 & $1.6 \mathrm{E}+00$ & $3.29 \mathrm{E}-02$ \\
\hline & & & & & & & 12 & 94.9 & $1.2 E+05$ & $3.53 \mathrm{E}-03$ \\
\hline P08476 & INHBA * & yes & & & & & 38 & 66.8 & $-9.4 E+00$ & $1.62 \mathrm{E}-02$ \\
\hline P24592 & IGFBP6 & yes & & & & & 19 & 36.5 & $-2.3 E+00$ & 7.44E-02 \\
\hline & & & & & & & 19 & 38.9 & $-3.9 E+05$ & 1.16E-02 \\
\hline Q96AY3 & FKBP10 & NR & & & & & 33 & 132.4 & $-3.4 E+04$ & 7.43E-05 \\
\hline & & & & & & & 34 & 125.5 & $1.7 E+00$ & $9.20 \mathrm{E}-02$ \\
\hline P05121 & SERPINE1 & yes & & & & & 45 & 69.55 & $-1.8 \mathrm{E}+00$ & $6.29 \mathrm{E}-02$ \\
\hline P36955 & SERPINF1 & yes & 12 & 54.5 & $-2.14 E+04$ & 2.73E-03 & 30 & 72.0 & $-1.6 E+00$ & 2.85E-02 \\
\hline & & & & & & & 30 & 70.3 & $-1.2 E+04$ & 1.27E-02 \\
\hline & & & & & & & 30 & 69.5 & $-8.3 E+03$ & $2.79 \mathrm{E}-02$ \\
\hline P50454 & SERPINH1 & NR & & & & & 49 & 68.8 & 4.4E-05 & 6.17E-03 \\
\hline P02768 & ALB & yes & 16 & 85.6 & $3.88 \mathrm{E}+00$ & 1.92E-02 & & & & \\
\hline & & & 16 & 83.8 & 3.17E+00 & 2.41E-02 & & & & \\
\hline & & & 16 & 83.8 & $3.77 E+00$ & $2.42 \mathrm{E}-02$ & & & & \\
\hline & & & 16 & 53.3 & $-2.71 E+04$ & 1.09E-02 & & & & \\
\hline P02787 & TF & yes & 20 & 93.9 & $-1.09 E+04$ & $3.66 \mathrm{E}-02$ & & & & \\
\hline Q7Z7G0 & $\mathrm{ABI} B \mathrm{BP}$ * & yes & & & & & 1 & 290.5 & $2.8 E+01$ & $9.26 \mathrm{E}-03$ \\
\hline & & & & & & & 1 & 233.9 & $-3.8 E+04$ & $8.21 \mathrm{E}-05$ \\
\hline & & & & & & & 17 & 299.3 & $-1.5 E+05$ & $1.54 \mathrm{E}-03$ \\
\hline Q7Z7G0 & $\mathrm{ABI} 3 \mathrm{BP}^{*}$ & yes & & & & & 29 & 285.3 & $-1.6 \mathrm{E}+05$ & 2.03E-04 \\
\hline Q8NBS9 & TXNDC5 & yes & & & & & 20 & 74.9 & $-9.4 E+03$ & 7.31E-03 \\
\hline & & & & non- & sical secr & & & & & \\
\hline P62736 & ACTA2 & yes & & & & & 32 & 61.3 & $-3.8 E+00$ & 4.35E-02 \\
\hline P06733 & ENO1 & yes & & & & & 39 & 73.1 & $-3.0 \mathrm{E}+00$ & $9.22 \mathrm{E}-02$ \\
\hline P30049 & ATP5D & yes & & & & & 2 & 17.2 & $3.8 E+00$ & 4.94E-02 \\
\hline Q96CX2 & KCTD12 & NR & 4 & 39.9 & $-5.05 E+00$ & $3.10 \mathrm{E}-02$ & & & & \\
\hline P30101 & PDIA3 & yes & 13 & 67.7 & $-2.37 E+04$ & 1.33E-02 & & & & \\
\hline Q15293 & RCN1 & NR & 2 & 51.5 & $-2.13 E+00$ & 2.71E-02 & & & & \\
\hline Q01995 & TAGLN & yes & 28 & 22.4 & $-4.89 E+00$ & $9.91 \mathrm{E}-02$ & & & & \\
\hline P37802 & TAGLN2 & yes & 27 & 21.3 & $-4.54 E+01$ & 1.10E-03 & & & & \\
\hline Q15904 & ATP6AP* & NR & & & & & 4 & 26.3 & $8.7 E+00$ & 2.68E-02 \\
\hline P08670 & VIM & yes & 2 & 51.5 & $-2.13 E+00$ & 2.71E-02 & 8 & 25.2 & $-6.0 \mathrm{E}+01$ & $1.25 \mathrm{E}-04$ \\
\hline & & & & & tracellular & & & & & \\
\hline P05387 & RPLP2 & yes & & & & & 2 & 17.2 & $3.8 E+00$ & 4.94E-02 \\
\hline B4DI54 & cDNA & NR & 8 & 82.3 & $-3.22 E+00$ & 3.72E-02 & & & & \\
\hline & FLJ56386 & & & & & & & & & \\
\hline P60709 & ACTB & yes & 11 & 48.3 & $-1.95 E+01$ & 1.10E-04 & & & & \\
\hline P00325 & $\mathrm{ADH} 1 \mathrm{~B}$ & NR & 30 & 44.2 & $-3.45 E+00$ & 2.74E-02 & & & & \\
\hline Q3LXA3 & DAK & yes & & & & & 42 & 264.8 & $-3.2 E+04$ & 1.14E-04 \\
\hline P12277 & CKB & yes & 11 & 48.3 & $-1.95 E+01$ & 1.10E-04 & & & & \\
\hline P04080 & CSTB & yes & 21 & 9.9 & $-2.16 E+00$ & 6.38E-02 & & & & \\
\hline Q01469 & FABP5 & yes & 18 & 11.8 & $-2.63 E+00$ & 8.23E-02 & & & & \\
\hline P04075 & ALDOA & yes & 29 & 47.2 & $-7.65 E+00$ & 7.47E-02 & & & & \\
\hline & & & 30 & 44.2 & $-3.45 E+00$ & 2.74E-02 & & & & \\
\hline P62879 & GNB2 & yes & & & & & 31 & 39.0 & $-3.8 E+04$ & $7.28 \mathrm{E}-03$ \\
\hline P11142 & HSPA8 & yes & 5 & 87.2 & $-2.54 E+00$ & 4.92E-02 & 34 & 125.5 & $1.7 \mathrm{E}+00$ & $9.20 \mathrm{E}-02$ \\
\hline & & & 8 & 82.3 & $-3.22 E+00$ & $3.72 \mathrm{E}-02$ & 35 & 124.6 & $-4.0 \mathrm{E}+00$ & $9.28 \mathrm{E}-02$ \\
\hline P62937 & PPIA & yes & & & & & 43 & 18.0 & $-9.4 E+00$ & 4.41E-02 \\
\hline P00558 & PGK1 & yes & 29 & 47.2 & $-7.65 E+00$ & 7.47E-02 & & & & \\
\hline P02545 & LMNA & yes & 22 & 78.4 & $-3.42 E+00$ & 7.94E-02 & & & & \\
\hline
\end{tabular}


Table 1: Identification of secreted proteins of $\mathrm{CoCl}_{2}$-treated or non-treated (pre)adipocytes

\begin{tabular}{|c|c|c|c|c|c|c|c|c|c|c|}
\hline \multirow{2}{*}{$\begin{array}{c}\text { accession } \\
\text { number }\end{array}$} & \multirow[t]{2}{*}{ protein } & \multirow{2}{*}{$\begin{array}{l}\text { Exo } \\
\text { Carta }\end{array}$} & \multicolumn{4}{|c|}{ adipocytes } & \multicolumn{4}{|c|}{ preadipocytes } \\
\hline & & & ID & $\begin{array}{c}\mathrm{MW} \\
{[\mathrm{kDa}]}\end{array}$ & $\begin{array}{c}\text { Fold } \\
\text { change }\end{array}$ & $p$-value & ID & $\begin{array}{c}\mathrm{MW} \\
{[\mathrm{kDa}]}\end{array}$ & $\begin{array}{c}\text { Fold } \\
\text { change }\end{array}$ & $p$-value \\
\hline \multicolumn{11}{|c|}{ intracellular } \\
\hline P07737 & PFN1 & yes & & & & & 48 & 16.0 & $1.8 \mathrm{E}+00$ & $3.80 \mathrm{E}-02$ \\
\hline P14618 & PKM & yes & & & & & 47 & 101.1 & $-7.1 \mathrm{E}+03$ & $1.86 \mathrm{E}-04$ \\
\hline P38646 & HSPA9 & yes & 8 & 82.3 & $-3.22 \mathrm{E}+00$ & $3.72 \mathrm{E}-02$ & & & & \\
\hline P55072 & VCP & yes & 6 & 102.7 & $-4.78 \mathrm{E}+00$ & 3.62E-02 & & & & \\
\hline O75347 & TBCA & yes & & & & & 18 & 16.8 & $-4.8 \mathrm{E}+00$ & $6.94 \mathrm{E}-02$ \\
\hline P54727 & RAD23B & NR & & & & & 13 & 92.0 & $9.6 \mathrm{E}+04$ & $1.55 \mathrm{E}-04$ \\
\hline \multicolumn{11}{|c|}{$\begin{array}{l}\text { Table 1: Identification of secreted proteins of } \mathrm{CoCl}_{2} \text {-treated or -non-treated (pre)adipocytes by } 2 \mathrm{DE} \text { followed by } \\
\text { LC-MS/MS. ID numbers refer to marked spots in Figure } 2 \text { and } 3 \text {. Proteins within the same category with the } \\
\text { identical ID numbers are related to the same spots and proteins marked with * are identified as nove } \\
\text { (pre)adipocyte secreted proteins. MW of the protein spots are defined by PDQuest } 8.0 \text { software. Identification as } \\
\text { known exosomal proteins is shown as yes by additional ExoCarta analyses. NR describes no results found with } \\
\text { the ExoCarta analysis. }\end{array}$} \\
\hline
\end{tabular}

The preadipocyte experiments showed 397 matched spots. 118 of these were differentially expressed, either significantly (99) or as a trend (19). From these 118 spots 101 were identified which revealed 48 unique proteins. By using SignalP v3.0 and SecretomeP v2.0 these proteins were sub-classified in classical secreted (34), non-classical secreted (5) and intracellular (9). The classical secreted proteins were further sub-categorized in ECM, processing and regulation/signaling proteins. Within the group of classical secreted proteins, collagens were prominently present. Especially, collagen alpha-1(I) was identified in 16 different single spots and in 30 different mixed spots. Most of the preadipocyte secreted proteins were down-regulated too. Only galectin 1 and $\mathrm{N}$-terminal collagen alpha-1(I) fragments were induced by $\mathrm{CoCl}_{2}$-treatment. In total 11 proteins were not known as secreted proteins and did not show any secretion feature (Table 1, Figure 3, Supplement 2).

Additional ExoCarta analysis confirmed 42 of all classical and non-classical (pre)adipocytes secreted proteins as exosomal proteins. As such, all ECM as well as processing proteins are described as exosomal proteins. Within the category of regulation/signalling and nonclassical secreted 14 of 21 and 7 of 10 proteins are mentioned as exosomal proteins. 
A
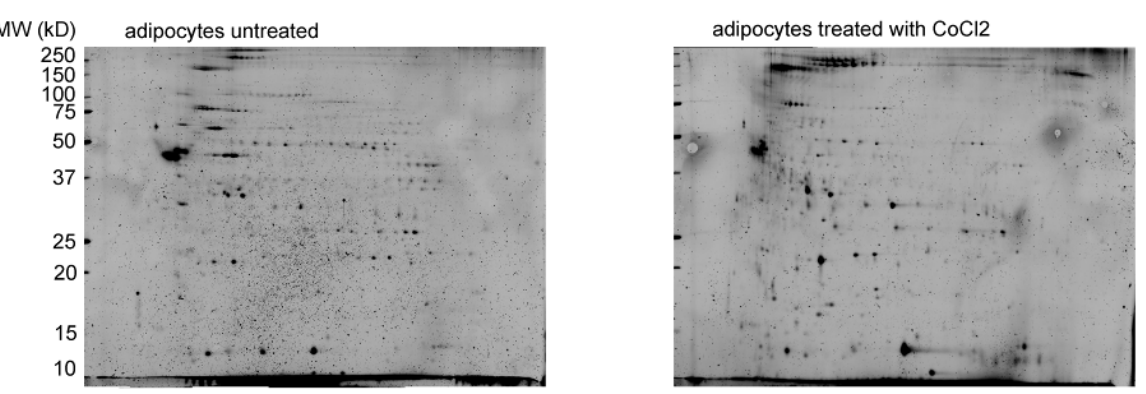

B

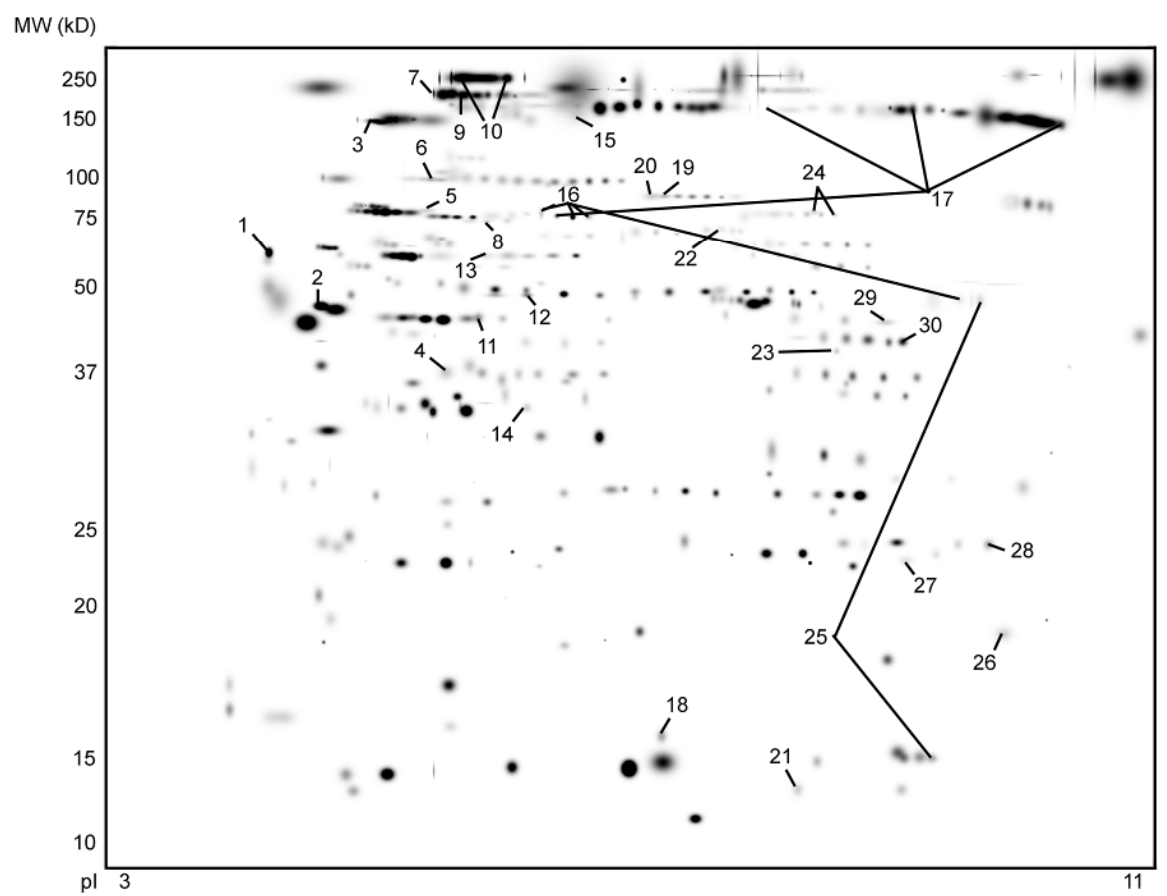

Figure 2: 2-DE gels of adipocyte secreted proteins. A: Representative gels of medium-derived secreted proteins of 13 days differentiated adipocytes after $48 \mathrm{~h}$ in the absence (left) or presence (right) of $100 \mu \mathrm{M} \mathrm{CoCl}_{2}$. B: Master gel with identified spots. Spot numbers refer to ID number of Table 1 and in Supplement 1

\section{Protein turnover in $\mathrm{CoCl}_{2}$ treated preadipocytes and adipocytes}

Hypoxia inhibits protein synthesis [32]. To investigate whether this influenced the (pre)adipocyte secretome, stable isotope labeled phenylalanine incorporation rates in secreted proteins were analyzed after $48 \mathrm{~h} \mathrm{CoCl}_{2}$ incubation. Initial tests showed the highest phenylalanine replacement values at this time point (data not shown).

In the secretome of $\mathrm{CoCl}_{2}$-treated and non-treated (pre)adipocytes no significant differences in the phenylalanine labeling percentages were found (Supplement 3). Some of the classical secreted proteins including $72 \mathrm{kDa}$ type IV collagenase precursor, apolipoprotein $\mathrm{E}$ precursor (ApoE), collagen alpha-2(I), collagen alpha-1(VI), cystatin $C$ precursor, metalloproteinase inhibitor 1 precursor, SPARC and target of Nesh-SH3 precursor, were consistently labeled. In contrast, collagen alpha-1(I) was identified only a few times as labeled and one collagen 
alpha-1(I) of $\mathrm{CoCl}_{2}$-treated adipocytes showed no labeling. Collagen alpha-1(III) and galactin1 were not labeled at all despite they contain phenylalanine in their sequence. Some proteins, like complement factor $\mathrm{D}$, were not labeled since they do not contain phenylalanine (Supplement 3). Overall, adipocytes compared to preadipocytes expressed higher levels of labeled proteins. On the other hand, the labeling rates of ApoE and collagen alpha-1(I) within non-treated (pre)adipocytes were increased compared to the $\mathrm{CoCl}_{2}$-treated cells (Supplement 3).

A
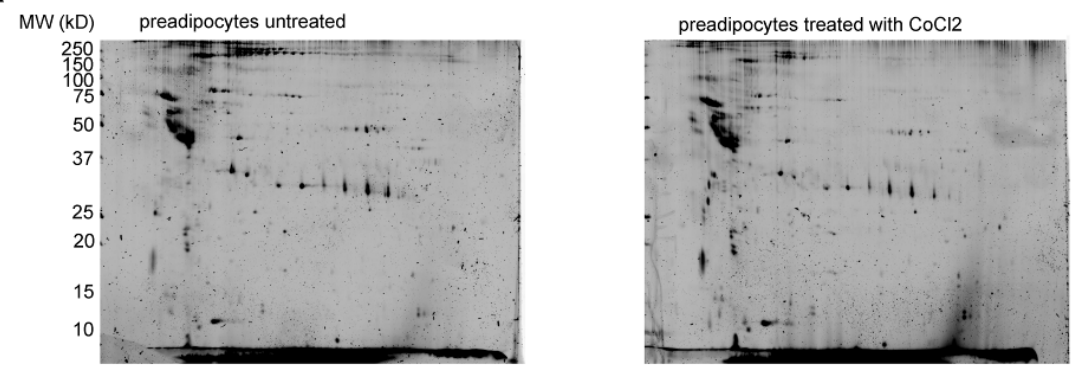

B

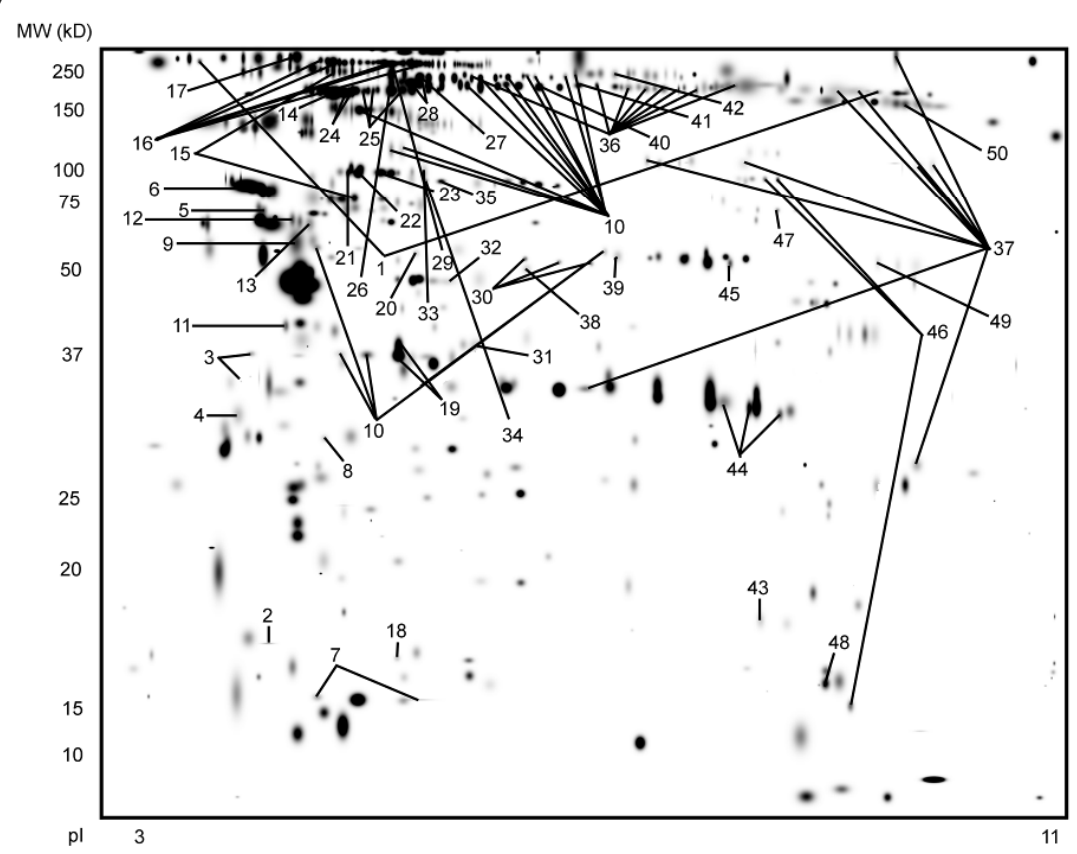

Figure 3. 2-DE gels of preadipocyte secreted proteins. A: Representative gels of mediumderived secreted proteins of up to $90 \%$ confluent preadipocytes in the absence (left) or presence (right) of $100 \mu \mathrm{M} \mathrm{CoCl}_{2}$. B: Master gel with identified spots. Spot numbers refer to ID number of Table 1 and in Supplement 1.

\section{Validation of the identified proteins}

Comparison of both 2DE data sets with previous human and rodent (pre)adipocyte secretome studies (reviewed by Lehr et al. [33]) identified inhibin beta A chain, v-type proton ATPase subunit S1 and cDNA FLJ55606, highly similar to alpha-2-HS-glycoprotein as novel (pre)adipocyte-expressed proteins. The proteins calumenin and target of Nesh-SH3 were often detected. However, specific isoforms have not been distinguished before in the (pre)adipocyte proteome, except for isoform 1 of calumenin [34]. Here, we have identified 


\section{CHAPTER 5}

calumenin isoform 4 and target of Nesh-SH3 isoforms 1 and 2. As such, 6 novel secreted proteins regulated by $\mathrm{CoCl}_{2}$ in (pre)adipocytes were identified. Protein identification of the turnover experiments revealed 3 additional novel (pre)adipocyte secreted proteins: carbohydrate sulfotransferase 5, HERV-K_5q33.3 provirus Np9 protein and telethonin (Table 1, Supplement 1, 2 and 3). In total this study revealed 9 novel (pre)adipocyte secreted proteins. The additional ExoCarta analyses showed that 4 of these 9 proteins were exosomal proteins.

\section{Effect of $\mathrm{CoCl}_{2}$ on lipolysis}

Hypoxia influences lipid metabolism in adipocytes [10], increases lipolysis in mouse 3T3-L1 adipocytes [35] and may negatively influence protein synthesis by HIF-1 $\alpha$-mediated ATP reduction [9]. Therefore, the effect of $\mathrm{CoCl}_{2}$ on lipolysis in human SGBS adipocytes was analyzed. No changes in TG accumulation were observed. In contrast, $48 \mathrm{~h} \mathrm{CoCl}_{2}$-treated adipocytes showed a significantly increased glycerol release (Figure 4A). This was further investigated by additional time and concentration studies, which revealed a positive correlation with glycerol release and $\mathrm{CoCl}_{2}$ concentrations (significant at $200 \mu \mathrm{M} \mathrm{CoCl}$ ) (Figure 4B). Again, no effect on TG accumulation was measured. The time course study indicated increased glycerol release starting from $16 \mathrm{~h}$ (not significant), but no effect on TG accumulation (Figure 4C).

\section{Discussions}

In this study we analyzed the hypoxia-mimetic effect of $\mathrm{CoCl}_{2}$ on the secretome of human SGBS (pre)adipocytes. SGBS cells are morphologically, biochemically and functionally similar to primary preadipocytes even after 30 generations, which is a definitive advantage compared to primary human fat cells [20].

The oxygen tension is crucial factor for WAT function [4, 14]. Hypoxic conditions activate members of the HIF family including HIF-1, HIF-2 and HIF-3. HIF-1 is a dominant hypoxiainduced factor, while HIF-2 and HIF-3 are more related to inflammatory responses [36, 37]. HIF-1 is composed of subunit $\alpha$ and $\beta$. While HIF-1 $\beta$ is constantly present, HIF-1 $\alpha$ is stabilized under low $\mathrm{O}_{2}$ tension to function as transcription factor $[17,38] . \mathrm{CoCl}_{2}$ is known to stabilize HIF-1 $\alpha$ and as such we used this compound to investigate hypoxia-mimetic effects on the SGBS (pre)adipocyte secretome. Challenging the SGBS cells for 48 hrs with $\mathrm{CoCl}_{2}$ resulted in a down-regulation of most of the differentially secreted proteins. In addition, 9 unknown (pre)adipocyte secreted proteins were identified.

Hypoxic cells convert to an anaerobic energy metabolism which includes the less efficient anaerobe glycolysis resulting in reduced ATP production by decreased mitochondrial activity 
[9]. An increased glycolytic flux and a glucose-to-lipid converted metabolism can be regulated via the HIF-1a-controlled glycerol-3-phosphate pathway [10]. Reduced ATP production negatively influences energy-dependent protein synthesis and secretion processes, which is shown here by $\mathrm{CoCl}_{2}$-mediated down-regulation of many (pre)adipocytesecreted proteins. We measured glycerol release and TG contents to investigate metabolic changes that may explain this down-regulation. $\mathrm{CoCl}_{2}$-treated adipocytes showed increased glycerol release without lowered TG contents. This agrees with a previous study [35] showing increased glycerol release from and decreased free fatty acid uptake in hypoxic mouse 3T3-L1 adipocytes. This was accompanied by reduced protein expression.

A
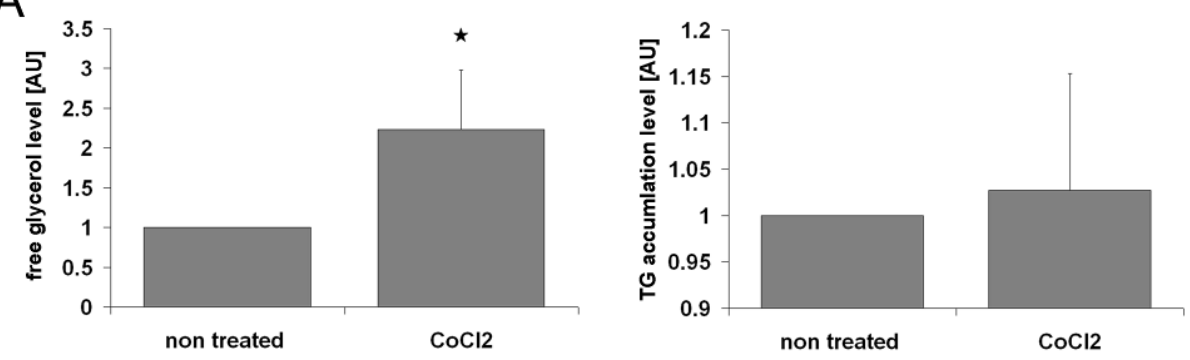

$\mathrm{B}$
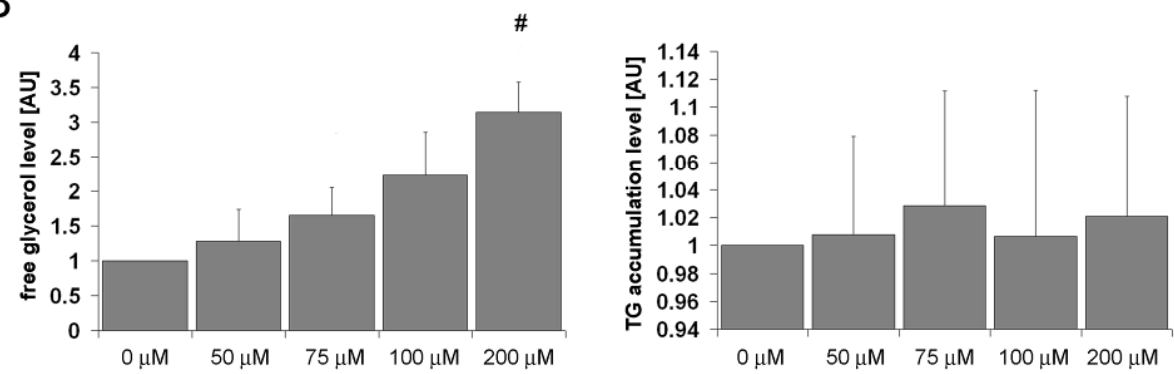

C
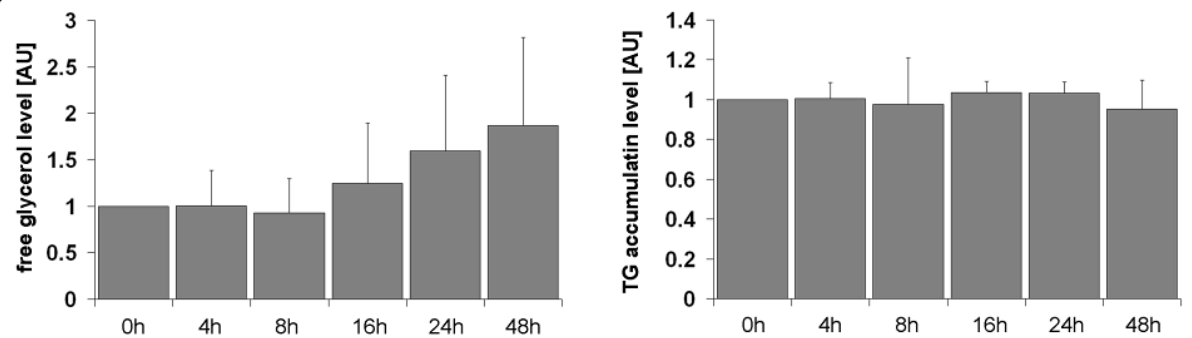

Figure 4: Effect of $\mathrm{CoCl}_{2}$ on lipolysis in adipocytes. A: Glycerol release (left) and TG accumulation (right) of $100 \mu \mathrm{M} \mathrm{CoCl}_{2}$-treated adipocytes after $48 \mathrm{~h}$ incubation. The reported values are means \pm $\mathrm{SD}$ of at least 5 biological replicates $(\mathrm{n} \geq 5)$ and * indicates differences with $p<0.05$ (Student T-test) B: Glycerol release and TG accumulation of a $48 \mathrm{~h} \mathrm{CoCl}_{2}$ concentration study with $0,50,75,100$ and $200 \mu \mathrm{M} \mathrm{CoCl}_{2}$ treatments. C: Glycerol release and TG accumulation during $4 \mathrm{~h}, 8 \mathrm{~h}, 16 \mathrm{~h}, 24 \mathrm{~h}$ and $48 \mathrm{~h}$ incubation with $100 \mu \mathrm{M} \mathrm{CoCl}_{2}$. All reported values are means \pm SD of at least 3 biological replicates $(n \geq 3)$ and \# indicates differences with $p<0.05$ (repeated measures ANOVA with Bonferroni post-hoc test). 


\section{CHAPTER 5}

Hypoxia-mediated reduction of protein turnover rates provides an additional mechanism for reduced protein synthesis and secretion. We observed higher rates of stable isotope labeled phenylalanine containing proteins in adipocytes compared to preadipocytes. This may rely on structural remodeling and fat accumulation during differentiation. However, compared to control conditions, labeling of $\mathrm{ApoE}$ and collagens in adipocytes was decreased. In addition, collagen alpha-1(I) showed a $50 \%$ increased labeling in control preadipocytes compared to $\mathrm{CoCl}_{2}$-treated cells. Decreased labeling rates in (pre)adipocytes indicate reduced protein synthesis and secretion. However, the flux of secretory proteins is dynamic due to constant release and synthesis of proteins. For instance, galectin-1 contains phenylalanine in its amino acid sequence but does not show labeling. This is probably due to slow protein turnover rates, which cannot be detected during the applied $48 \mathrm{~h} \mathrm{CoCl}_{2}$ incubation. This may also explain why some proteins with several phenylalanine-containing peptides only label one or two specific phenylalanine sites. For a complete protein turnover comparison not only the same protein but also all phenylalanine-containing peptides of this protein should be identified.

$\mathrm{CoCl}_{2}$-induced changes of the adipocyte secretome revealed a down regulation of most of the differentially expressed proteins. Only collagens and serum albumin were up-regulated. As ECM proteins collagens are important for structural remodeling of cells. An up-regulation of collagens by $\mathrm{CoCl}_{2}$ agrees with previous hypoxia studies with WAT [12, 13]. This characterizes WAT dysfunction and the associated development of adipose tissue fibrosis. Fibrotic adipocytes of obese subjects are related to insulin resistance [12, 39]. However, increased collagen levels may also reflect cell survival. The $\mathrm{CoCl}_{2}$-mediated down-regulation of other ECM-classified proteins is in contrast with previous hypoxia-induced gene expression studies $[12,40]$.. Such differences may be addressed to different cell species or to low correlation levels of RNA and protein expression. According to UniProtKB/Swiss-Prot calreticulin, fibronectin and beta ig-h3 are involved in ECM-cell interactions and not directly involved in the ECM fibrin structure remodeling as collagens are. Apparently, $\mathrm{CoCl}_{2}$ induces different regulation of various functional classes of ECM components including the preservation of (fibrillar) collagens in mature adipocytes.

Another $\mathrm{CoCl}_{2}$-induced protein is human serum albumin. Albumin is present in serumcontaining cell culture medium. However, we observed a significant up-regulation of human albumin within $\mathrm{CoCl}_{2}$-treated adipocytes grown in serum-devoid medium. Serum albumin is a carrier for fatty acids and, as such, increased glycerol release might be a sign for enhanced lipolysis and fatty acid release. However, ApoE, which is involved in lipid synthesis, $\beta$ oxidation and regulation of TG turnover [41] was down-regulated. Reduced ApoE may indicate decreased TG requirement during low $\beta$-oxidation capacity in an anaerobic 
metabolism. As such, down-regulated ApoE and stable fat accumulation may indicate decreased lipolysis.

Compared to adipocytes, preadipocytes have a different function and secretion profile [21]. However, they play an important role in the tightly regulated WAT metabolism. Hypoxia alters preadipocyte secreted factors [42] and inhibits preadipocyte differentiation by decreased levels of peroxisome proliferator-activated receptor-y (PPARY), [43]. This is in line with a hypoxia-mediated reduction of energy-dependent protein synthesis. $\mathrm{CoCl}_{2}$ down-regulated nearly all normal size ECM proteins in human preadipocytes. ECM proteins are crucial for preadipocyte remodeling during differentiation [44], which is inhibited by the down-regulation of full-length collagens. In contrast, $\mathrm{N}$-terminal collagen fragments were up-regulated probably by increased collagen processing rates since $\mathrm{C}$ and $\mathrm{N}$-terminal parts of precursor collagens are cut off during processing [45]. Furthermore, the presence of collagen alpha-1(I) and $-2(\mathrm{I})$ mixed spots may indicate $\mathrm{CoCl}_{2}$-induced collagen processing. Collagen alpha-1(I) preferentially connects with collagen alpha-2(I) and forms fibrils which cross-link to strong mature type 1 collagen fibers [46]. These are important components for preadipocyte remodeling during differentiation [44]. Insufficient amounts of precursor collagens and, as such, down-regulated full-length precursor collagens may thus limit preadipocyte differentiation.

Galectin-1 is a direct target of HIF-1a [47] and is up-regulated within $\mathrm{CoCl}_{2}$-treated preadipocytes. This might disagree with the assumption of inhibited preadipocyte differentiation, since galectin-1 induces trophoblast differentiation by proliferation inhibition [48]. In colorectal cancer cells HIF-1 1 induces galectin-1, which stimulates hypoxia-mediated migration and invasion of these cells [49]. Despite the hypoxia-mediated inhibition of preadipocyte differentiation, the $\mathrm{CoCl}_{2}$-induced galectin-1 expression found here may be countered. Galectin-1-induced cell differentiation may be an early event in preadipocyte differentiation, which is subsequently inhibited by the later action of down-regulated PPARY and collagens.

Since proteomics studies on hypoxia-induced changes of total human (pre)adipocyte secretomes are lacking, we compared our data with recently published microarray analysis of hypoxia-treated adipocytes [50]. In SGBS adipocytes 8 hypoxia-induced genes were identified, which were related to HIF transcription factor family members HIF1A and HIF2A [50]. However, none of their gene products was present within our data. Wang et al. [51] demonstrated a dysregulation of generally known adipokines under hypoxic conditions, but none of these proteins was identified in our study, probably due to their low abundance. In addition, we compared both 2DE and turnover data sets with recent adipocyte secretome studies [52]. This revealed 9 new adipocyte-secreted proteins of which 4 (calumenin isoform 4 , both targets of Nesh-SH3 isoforms and inhibin beta A chain) were verified as exosomal 


\section{CHAPTER 5}

proteins. The ExoCarta database is built with experimental results; as such, not all exosomal proteins are present yet. Particularly novel secretory proteins, as we also identified here, are lacking.

With respect to the 9 new adipocyte-secreted proteins, according to UniProtKB/Swiss-Prot carbohydrate sulfotransferase 5, and v-type proton ATPase subunit S1 are involved in general cell signaling and regulation processes and are not associated with adipocytespecific functions. The same is true for calumenin isoform 4, HERV-K_5q33.3 provirus Np9 protein and both targets of Nesh-SH3 isoforms. Telethonin is associated with the visual system and with muscle functions. Inhibin beta A chain belongs to the transforming growth factor beta family and regulates follicle-stimulating hormone secretion [53]. It also stimulates proliferation of embryonic cells and in turn regulates cell differentiation. Preadipocytes are also subjected to proliferation and differentiation processes which may be regulated by inhibin beta A. Down-regulated inhibin beta A decreases cell proliferation activity [54]. These findings may be in line with down-regulated preadipocyte collagen secretion. The cDNA FLJ55606, highly similar to alpha-2-HS-glycoprotein, is related to a cysteine-type endopeptidase inhibitor activity. Till now no secretion features are known for this protein. However, alpha-2-HS-glycoprotein, identified before as adipokine, is secreted by different types of cells $[21,55]$. Therefore, cDNA FLJ55606 protein, highly similar to alpha-2-HSglycoprotein, may have similar features as the alpha-2-HS-glycoprotein adipokine.

\section{Conclusion}

In conclusion, this study revealed 9 novel adipocyte-secreted proteins from which 6 were regulated by $\mathrm{CoCl}_{2}$. We showed specific down-regulation of proteins and $\mathrm{ECM}$ dysregulation in human preadipocytes and adipocytes. In adipocytes $\mathrm{CoCl}_{2}$ resulted in an up-regulation of collagens and a down-regulation of specific functional classes of ECM proteins. This may provide an essential adipocyte survival mechanism under hypoxic conditions. In preadipocytes, $\mathrm{CoCl}_{2}$ caused a down-regulation of proteins involved in fat cell differentiation. All these changes can be regarded as WAT dysfunction, which contributes to the initiation of obesity-associated complications.

\section{Acknowledgements}

This study was supported by a grant from the School of Life Sciences, transnationale Universiteit Limburg to JR. Dr. Martin Wabitsch (UIm University Medical Center, Germany) is kindly acknowledged for providing SGBS cells, Eric Royackers (University Hasselt, Belgium) 
for practical support and Dr. Johan Jocken (Maastricht University, The Netherlands) for helpful discussions. The PRIDE team is acknowledged for handling our MS data. This study is MIAPE compliant.

\section{References}

1. Gregoire, F.M., Adipocyte differentiation: from fibroblast to endocrine cell. Exp Biol Med (Maywood), 2001. 226(11): p. 997-1002.

2. Diamanti-Kandarakis, E., et al., The Impact of Endocrine Disruptors on Endocrine Targets. Horm Metab Res, 2010. 42(8): p. 543-52.

3. Pasarica, M., et al., Reduced Oxygenation in Human Obese Adipose Tissue Is Associated with Impaired Insulin Suppression of Lipolysis. J Clin Endocrinol Metab, 2010. 95(8): p. 4052-5.

4. Trayhurn, P. and I.S. Wood, Adipokines: inflammation and the pleiotropic role of white adipose tissue. Br J Nutr, 2004. 92(3): p. 347-55.

5. Virtanen, K.A., et al., Glucose uptake and perfusion in subcutaneous and visceral adipose tissue during insulin stimulation in nonobese and obese humans. J Clin Endocrinol Metab, 2002. 87(8): p. 3902-10.

6. Karpe, F., et al., Impaired postprandial adipose tissue blood flow response is related to aspects of insulin sensitivity. Diabetes, 2002. 51(8): p. 2467-73.

7. Lolmede, K., et al., Effects of hypoxia on the expression of proangiogenic factors in differentiated 3T3-F442A adipocytes. Int J Obes Relat Metab Disord, 2003. 27(10): p. 1187-95.

8. Skurk, T., et al., Relationship between adipocyte size and adipokine expression and secretion. J Clin Endocrinol Metab, 2007. 92(3): p. 1023-33.

9. Denko, N.C., Hypoxia, HIF1 and glucose metabolism in the solid tumour. Nat Rev Cancer, 2008. 8(9): p. 705-13.

10. Krishnan, J., et al., Activation of a HIF1alpha-PPARgamma axis underlies the integration of glycolytic and lipid anabolic pathways in pathologic cardiac hypertrophy. Cell Metab, 2009. 9(6): p. 512-24.

11. Trayhurn, P., B. Wang, and I.S. Wood, Hypoxia in adipose tissue: a basis for the dysregulation of tissue function in obesity? Br J Nutr, 2008. 100(2): p. 227-35.

12. Halberg, N., et al., Hypoxia-inducible factor 1alpha induces fibrosis and insulin resistance in white adipose tissue. Mol Cell Biol, 2009. 29(16): p. 4467-83.

13. Khan, T., et al., Metabolic dysregulation and adipose tissue fibrosis: role of collagen VI. Mol Cell Biol, 2009. 29(6): p. 1575-91. 
14. Famulla, S., et al., Differentiation of human adipocytes at physiological oxygen levels results in increased adiponectin secretion and isoproterenol-stimulated lipolysis. Adipocyte, 2012. 1(3): p. 132-181.

15. Goldberg, M.A., S.P. Dunning, and H.F. Bunn, Regulation of the erythropoietin gene: evidence that the oxygen sensor is a heme protein. Science, 1988. 242(4884): p. 1412-5.

16. Myllyharju, J., Prolyl 4-hydroxylases, master regulators of the hypoxia response. Acta Physiol (Oxf), 2013. 208(2): p. 148-65.

17. Semenza, G.L., Regulation of mammalian $\mathrm{O} 2$ homeostasis by hypoxia-inducible factor 1. Annu Rev Cell Dev Biol, 1999. 15: p. 551-78.

18. Majmundar, A.J., W.J. Wong, and M.C. Simon, Hypoxia-inducible factors and the response to hypoxic stress. Mol Cell, 2010. 40(2): p. 294-309.

19. Kaelin, W.G., Jr. and P.J. Ratcliffe, Oxygen sensing by metazoans: the central role of the HIF hydroxylase pathway. Mol Cell, 2008. 30(4): p. 393-402.

20. Wabitsch, M., et al., Characterization of a human preadipocyte cell strain with high capacity for adipose differentiation. Int J Obes Relat Metab Disord, 2001. 25(1): p. 815.

21. Rosenow, A., et al., Identification of Novel Human Adipocyte Secreted Proteins by Using SGBS Cells. J Proteome Res, 2010. 9: p. 5389-401.

22. Rosenow, A., et al., Resveratrol-induced changes of the human adipocyte secretion profile. J Proteome Res, 2012. 11(9): p. 4733-43.

23. Bouwman, F., J. Renes, and E. Mariman, A combination of protein profiling and isotopomer analysis using matrix-assisted laser desorption/ionization-time of flight mass spectrometry reveals an active metabolism of the extracellular matrix of 3T3-L1 adipocytes. Proteomics, 2004. 4(12): p. 3855-63.

24. Bradford, M.M., A rapid and sensitive method for the quantitation of microgram quantities of protein utilizing the principle of protein-dye binding. Anal Biochem, 1976. 72: p. 248-54.

25. Dumont, D., et al., Characterization of mature rat oligodendrocytes: a proteomic approach. J Neurochem, 2007. 102(2): p. 562-76.

26. Cranenburg, E.C., et al., The circulating inactive form of matrix Gla Protein (UCMGP) as a biomarker for cardiovascular calcification. J Vasc Res, 2008. 45(5): p. 427-36.

27. Vizcaino, J.A., et al., The PRoteomics IDEntifications (PRIDE) database and associated tools: status in 2013. Nucleic Acids Res, 2013. 41(Database issue): p. D1063-9.

28. Barsnes, H., et al., PRIDE Converter: making proteomics data-sharing easy. Nat Biotechnol, 2009. 27(7): p. 598-9. 
29. Vizcaino, J.A., et al., The Proteomics Identifications database: 2010 update. Nucleic Acids Res, 2010. 38(Database issue): p. D736-42.

30. Wang, R., et al., PRIDE Inspector: a tool to visualize and validate MS proteomics data. Nat Biotechnol, 2012. 30(2): p. 135-7.

31. Huang, L.E., et al., Regulation of hypoxia-inducible factor 1alpha is mediated by an O2-dependent degradation domain via the ubiquitin-proteasome pathway. Proc Natl Acad Sci U S A, 1998. 95(14): p. 7987-92.

32. Connolly, E., et al., Hypoxia inhibits protein synthesis through a 4E-BP1 and elongation factor 2 kinase pathway controlled by mTOR and uncoupled in breast cancer cells. Mol Cell Biol, 2006. 26(10): p. 3955-65.

33. Lehr, S., S. Hartwig, and H. Sell, Adipokines: A treasure trove for the discovery of biomarkers for metabolic disorders. Proteomics Clin Appl, 2012. 6(1-2): p. 91-101.

34. Molina, H., et al., Temporal profiling of the adipocyte proteome during differentiation using a five-plex SILAC based strategy. J Proteome Res, 2009. 8(1): p. 48-58.

35. Yin, J., et al., Role of hypoxia in obesity-induced disorders of glucose and lipid metabolism in adipose tissue. Am J Physiol Endocrinol Metab, 2009. 296(2): p. E33342.

36. Aragones, J., et al., Myeloid hypoxia-inducible factors in inflammatory diseases. Crit Rev Immunol, 2011. 31(1): p. 1-13.

37. Forooghian, F., R. Razavi, and L. Timms, Hypoxia-inducible factor expression in human RPE cells. Br J Ophthalmol, 2007. 91(10): p. 1406-10.

38. Cummins, E.P. and C.T. Taylor, Hypoxia-responsive transcription factors. Pflugers Arch, 2005. 450(6): p. 363-71.

39. Spencer, M., et al., Adipose Tissue Extracellular Matrix and Vascular Abnormalities in Obesity and Insulin Resistance. J Clin Endocrinol Metab, 2011. 96(12): p. E1990-8.

40. Distler, J.H., et al., Hypoxia-induced increase in the production of extracellular matrix proteins in systemic sclerosis. Arthritis Rheum, 2007. 56(12): p. 4203-15.

41. Huang, Z.H., et al., Nutritional regulation of adipose tissue apolipoprotein $E$ expression. Am J Physiol Endocrinol Metab, 2007. 293(1): p. E203-9.

42. Wang, B., I.S. Wood, and P. Trayhurn, Hypoxia induces leptin gene expression and secretion in human preadipocytes: differential effects of hypoxia on adipokine expression by preadipocytes. J Endocrinol, 2008. 198(1): p. 127-34.

43. Kim, K.H., et al., Hypoxia inhibits adipocyte differentiation in a HDAC-independent manner. Biochem Biophys Res Commun, 2005. 333(4): p. 1178-84.

44. Mariman, E.C. and P. Wang, Adipocyte extracellular matrix composition, dynamics and role in obesity. Cell Mol Life Sci, 2010. 67(8): p. 1277-92. 


\section{CHAPTER 5}

45. Trackman, P.C., Diverse biological functions of extracellular collagen processing enzymes. J Cell Biochem, 2005. 96(5): p. 927-37.

46. Robins, S.P., Biochemistry and functional significance of collagen cross-linking. Biochem Soc Trans, 2007. 35(Pt 5): p. 849-52.

47. Zhao, X.Y., et al., Synergistic induction of galectin-1 by CCAAT/enhancer binding protein alpha and hypoxia-inducible factor 1alpha and its role in differentiation of acute myeloid leukemic cells. J Biol Chem, 2011. 286(42): p. 36808-19.

48. Fischer, I., et al., The role of galectin-1 in trophoblast differentiation and signal transduction. J Reprod Immunol, 2011. 90(1): p. 35-40.

49. Li, H.X., et al., Review: Epigenetic regulation of adipocyte differentiation and adipogenesis. J Zhejiang Univ Sci B, 2010. 11(10): p. 784-91.

50. Beck, F., et al., The good, the bad, the ugly: Validating the mass spectrometric analysis of modified peptides. Proteomics, 2011. 11(6): p. 1099-109.

51. Wang, B., I.S. Wood, and P. Trayhurn, Dysregulation of the expression and secretion of inflammation-related adipokines by hypoxia in human adipocytes. Pflugers Arch, 2007. 455(3): p. 479-92.

52. Famulla, S., et al., Pigment epithelium-derived factor (PEDF) is one of the most abundant proteins secreted by human adipocytes and induces insulin resistance and inflammatory signaling in muscle and fat cells. Int J Obes (Lond), 2011. 35(6): p. 76272.

53. Vale, W., et al., Purification and characterization of an $\mathrm{FSH}$ releasing protein from porcine ovarian follicular fluid. Nature, 1986. 321(6072): p. 776-9.

54. Tomaszewski, J., et al., Essential roles of inhibin beta $A$ in mouse epididymal coiling. Proc Natl Acad Sci U S A, 2007. 104(27): p. 11322-7.

55. Ix, J.H. and K. Sharma, Mechanisms linking obesity, chronic kidney disease, and fatty liver disease: the roles of fetuin-A, adiponectin, and AMPK. J Am Soc Nephrol, 2010. 21(3): p. 406-12. 


\section{Chapter 6}

\section{Resveratrol-induced changes of the human adipocyte secretion profile}

Anja Rosenow, Jean-Paul Noben, Johan Jocken, Sonja Kallendrusch, Pamela FischerPosovszky, Edwin C.M. Mariman, Johan Renes

Journal of Proteome Research. 2012; 9 4733-4743 


\title{
CHAPTER 6
}

\begin{abstract}
Enlarged white adipose tissue (WAT) is a feature of obesity and leads to changes in its paracrine and endocrine function. Dysfunction of WAT cells is associated with obesityassociated disorders like type 2 diabetes and cardiovascular diseases. Resveratrol (RSV) a natural polyphenolic compound mimics beneficial effects of calorie restriction. As such, RSV seems a promising therapeutic target for obesity-associated disorders. The effect of RSV on the human adipokine profile is still elusive. Therefore, a proteomic study together with bioinformatical analysis was performed to investigate the effect of RSV on the secretion profile of mature human SGBS adipocytes. RSV incubation resulted in elevated basal glycerol release and reduced intracellular TG content. This increased intracellular lipolysis was accompanied by profound changes in the adipocyte secretion profile. Extracellular matrix proteins were down-regulated while processing proteins were mostly up-regulated after RSV treatment. Interestingly, RSV induced secretion of proteins protective against cellular stress and proteins involved in the regulation of apoptosis. Furthermore, we found a RSV-induced up-regulation of adiponectin and ApoE accompanied by a down-regulation of PAI-1 and PEDF secretion which may improve anti-inflammatory processes and increased insulin sensitivity. These effects may contribute to alleviate obesity-induced metabolic complications. In addition, two novel RSV-regulated adipocyte-secreted proteins were identified.
\end{abstract}




\section{Introduction}

The polyphenol resveratrol (RSV) (3,4',5-trihydroxystilbene) is synthesized in more than 70 plant species and is present in many dietary substances [1, 2]. Animal studies have shown that RSV mimics several beneficial effects of calorie restriction including prevention of insulin resistance, increased mitochondrial contents of several tissues like liver and skeletal muscles as well as the restoration of normal longevity in obese mice [1, 3, 4]. However, despite other benefits from calorie restriction such as slowing heart rate, prevention of spontaneous lymphomas, decreased core body temperature RSV-treatment did not extend the lifespan in non-obese animals [1, 3, 4]. Recently, Timmers et al. [5] and Olholm et al. [6] have demonstrated that RSV can mimic positive effects of calorie restriction in obese humans. These included improvement of insulin resistance, decreased levels of blood glucose, triglycerides and cytokines as well as decreased intramyocellular lipid levels, decreased intrahepatic lipid content, and decreased systolic blood pressure [5, 6]. However, the molecular pathways by which RSV executes its beneficial effect are still poorly known.

In mammals, most of the beneficial effects of RSV treatment are mediated by the activation of sirtuin (SIRT) 1 [7]. SIRT1 is the mammalian homologue of the yeast Sir2 protein, which is involved in the longer lifespan of yeast upon calorie restriction [8]. However, it is still under discussion if RSV activates SIRT1 in a direct or indirect manner. Recently published data demonstrate a SIRT1 activation as a downstream signaling effect of a RSV-stimulated AMPactivated protein kinase (AMPK) activation [1, 9] In addition, Park et. al. [10] showed cAMP effector protein Epac1 as a key regulator of the RSV-induced effects which in turn activates AMPK and SIRT1.

Obesity and its associated diseases like type 2 diabetes, cardiovascular complications and certain cancers are currently major health burdens. Obesity is characterized by morphological, histological and functional changes of accumulated white adipose tissue (WAT). The accumulation of WAT is associated with an increase of adipocyte number (hyperplasia) and/or with an enlargement of existing adipocytes (hypertrophy) [11]. Simultaneously, changes of the paracrine and endocrine functions of WAT are related to changes in the protein expression and secretion profiles of (pre)adipocytes. Hormones, cytokines, neurotrophins, extracellular matrix proteins as well as proteins involved in angiogenesis, inflammation, lipid and glucose metabolism are known secreted factors of WAT and a dysregulation of their secretion pattern may lead to obesity-associated disorders $[12,13]$.

Previously, we investigated changes in the secretion profiles during fat cell differentiation by using human Simpson Golabi Behmel Syndrome (SGBS) adipocytes [14]. Here we hypothesized that RSV may have a beneficial effect on the secretion profile of mature human SGBS adipocytes. Therefore, the differences of the secretome of fully differentiated RSV- 


\section{CHAPTER 6}

treated or non-treated SGBS adipocytes were analyzed by two-dimensional gel electrophoresis (2-DE). The identification of the secreted proteins was performed by liquid chromatography-electrospray ionization tandem mass spectrometry (LC-ESI-MS/MS).

\section{Methods}

\section{Materials}

Culture media, $0.5 \%$ trypsin-EDTA and 10,000 u/ml Penicillin/Streptomycin were obtained from Life Technologies (Bleiswijk, The Netherlands). Fetal bovine serum (FBS) was purchased from Bodinco (Alkmaar, The Netherlands). Additional cell culture supplements, RSV, protease inhibitor cocktail, phenylmethylsulfonylfluoride (PMSF), DL-dithiothreitol (DTT), 3-[(3-cholamidopropyl) dimethyl-amonio]-1-propanesulfonate (CHAPS), a-cyano-4hydroxyl-cinnamic acid (CHCA), trifluoroacetic acid (TFA) and acetonitrile (ACN) were obtained from Sigma-Aldrich (Zwijndrecht, The Netherlands). Immobilized pH gradient (IPG) buffer ( $\mathrm{pH}$ 3-11, nonlinear), Dry-Strip cover fluid and immobiline Dry-Strips $(\mathrm{pH} 3-11$ nonlinear, $24 \mathrm{~cm}$ ) were purchased from GE Healthcare (Diegem, Belgium).

\section{Cell culture}

Human Simpson-Golabi-Behmel syndrome (SGBS) cells were obtained from Prof. Dr. M. Wabitsch (University of Ulm, Germany) [15] and cultured as described [14]. 90\% confluent preadipocytes $\left(2.2 \pm 0.36 \times 10^{6}\right.$, mean $\left.\pm S E M, n=3\right)$ were differentiated into mature adipocytes during 13 days as described [14]. On average $83 \%$ of the preadipocytes differentiated into mature adipocytes. To determine cell numbers, adipocytes were counted with a raster ocular. Preadipocytes were trypsinized and counted with a haemocytometer. The number of adipocytes per $150 \mathrm{~mm}$ Petri dish was $1.8 \pm 0.95 \times 10^{6}$ (mean $\pm \mathrm{SEM}, \mathrm{n}=7$ ).

\section{RSV experiments}

Dose-response experiment was performed to define the optimal RSV concentration to study its effect on the human adipocyte secretome. Briefly, 13 days differentiated adipocytes were treated with $66 \mathrm{nM}$ insulin and with different RSV concentrations $(0,5,50,75,100$ and 200 $\mu \mathrm{M}$ ) in phenol red free DMEM/F12 medium for a period of $48 \mathrm{~h}$. To study RSV-induced changes in the adipocyte secretome, fully differentiated adipocytes were washed twice with PBS and incubated for $48 \mathrm{~h}$ in phenol red free DMEM/F12 medium with $66 \mathrm{nM}$ insulin and $75 \mu \mathrm{M}$ RSV. Control adipocytes were cultured in a similar manner with $66 \mathrm{nM}$ insulin and an equal volume of dimethylsulfoxide (DMSO) instead of RSV. 


\section{Effect of RSV on lipolysis}

To determine the effect of $48 \mathrm{~h}$ RSV treatment $(75 \mu \mathrm{M})$ on intracellular lipolysis in human SGBS adipocytes, intracellular triglyceride (TG) content and glycerol release (marker of complete TG hydrolysis) were determined. Adipocytes were fixed with $3.7 \%$ formaldehyde for $10 \mathrm{~min}$ at room temperature. Fixed cells were incubated with a filtered Oil Red O (ORO) solution (1\% ORO in $60 \%$ isopropanol) for $30 \mathrm{~min}$. Finally, cells were washed 6 times with $70 \%$ ethanol. Images were taken with a Nikon TE 200 eclipse phase contrast microscope equipped with digital image acquisition. To determine intracellular TG accumulation, the ORO stain was extracted with DMSO and measured by spectrophotometry at $540 \mathrm{~nm}$. The amount of intracellular ORO content was expressed per number of cells.

Glycerol in the culture medium was measured by a fluorimetric assay using a Cobas-Fara Instrument (Roche Diagnostics, Almere, The Netherlands). The amount of glycerol release was expressed per number of cells.

\section{Sample preparation of 2-DE experiments}

Culture media were collected, supplemented with protease inhibitor cocktail, transferred to dialysis tubes (2-kDa molecular-weight cut-off, Carl Roth $\mathrm{GmbH}$, Karlsruhe, Germany) and dialyzed against $20 \mathrm{mM}$ ammonium bicarbonate at $4^{\circ} \mathrm{C}$ for $48 \mathrm{~h}$ with $10-12 \mathrm{x}$ buffer changes. The dialyzed medium samples were freeze-dried, dissolved in fresh rehydration buffer ( $8 \mathrm{M}$ urea, $2 \% \mathrm{w} / \mathrm{v}$ CHAPS and $65 \mathrm{mM} \mathrm{DTT}$ ) and centrifuged at $20000 \mathrm{x} \mathrm{g}$ at $10^{\circ} \mathrm{C}$ for $30 \mathrm{~min}$. The protein concentrations were determined with a Bradford-based protein assay kit (Bio-Rad Laboratories). All samples were stored at $-80^{\circ} \mathrm{C}$.

\section{2-DE}

Protein samples derived from $1.8 \times 10^{6}$ cells were used for 2-DE gel analysis according to Bouwman et al. [16] but with different IPG strips ( $\mathrm{pH}$ 3-11 nonlinear, $24 \mathrm{~cm}$ ). $100 \mu \mathrm{g}$ of total protein in a volume of $450 \mu$ containing $0.5 \%(v / v)$ IPG buffer was loaded onto the IPG strips. For protein profiling, 5 independent replicates were made for RSV-treated and non-treated adipocytes. The gels were stained with Flamingo fluorescent gel stain according to the manufacturer's protocol (Bio-Rad Laboratories) and scanned by the Molecular Imager FX (Bio-Rad Laboratories) to visualize the proteins.

\section{Image analysis}

Stained gels were processed by PDQuest 8.0 (Bio-Rad Laboratories). Data were normalized with respect to the total density of the gel image. The groups of RSV-treated and non-treated adipocytes were formed from samples of independent experiments with the same treatments. Protein spots were regarded as significantly differentially expressed if the 
average spot intensity between the groups differed more than 1.5 -fold with $p<0.05$ (Student's t-test). The criteria for indicating a trend was a spot intensity difference of more than 1.5-fold and $0.05<p<0.1$. For subsequent protein identification, gels with differentially expressed spots were re-stained with SYPRO Ruby Protein Stain according to the manufacturer's protocol (Bio-Rad Laboratories).

\section{MS/MS and protein identification}

Excised spots were in-gel digested as described by Bouwman et al. [16]. $10 \mu$ of the digested spots were analyzed by LC-ESI MS/MS on a LCQ Classic (ThermoFinnigan, San Jose, USA), as described by Dumont et al. [17]. The trapped sample was separated on the analytical column (Biosphere C18, $5 \mu \mathrm{m}$ particle diameter, $200 \mathrm{~mm} \mathrm{~L} \times 0.05 \mathrm{~mm}$ ID; Nanoseparations, Nieuwkoop, The Netherlands) using a linear gradient from 5 to $60 \% \mathrm{v} / \mathrm{v}$ $\mathrm{ACN}$ in water containing $100 \mathrm{mM}$ acetic acid in $55 \mathrm{~min}(100 \mathrm{nl} / \mathrm{min})$. The eluate of the analytical column was nanosprayed from a teflon-connected, gold-coated fused silica emitter (5 $\mu \mathrm{m}$ ID; NanoSeparations).

\section{Database search}

With respect to LC-ESI-MS/MS, LCQ Xcalibur v2.0 SR2 raw files and spectra were selected from Proteome Discover1.2 software (Thermo Scientific) with the following settings: minimal peak count 50; total intensity threshold 4000; and $S / N \geq 6$. Peak lists were searched with Sequest v1.2.0.208 and Mascot v2.3.0.1 against EMBL-EBI International Protein Index database for human proteins (version 3.78, 86702 entries) and using following settings: fragment tolerance, 1.00 Da (monoisotopic); parent tolerance, 3.0 Da (monoisotopic); fixed modifications, carbamidomethylation of cystein; variable modifications, oxidation of methionin; max missed cleavages, 2. Search engine results were combined and validated by Scaffold v3.00.07 (Proteome Software, Portland, OR, USA) with minimum peptide and protein probability set to $\geq 80 \%$, followed by visual inspection of spectral (annotation) quality and manual curation, eliminating high-background spectra and single-engine identifications, evaluating one-hit wonders and filtering out keratins. Finally, the Mascot DAT files were processed by PRIDE Converter software [18] and were submitted together with relevant files (.raw, .mgf, .dat and Scaffold .sf3 files) via ProteomeXchange (http://www.protemexchange.org) to the PRIDE database [19], project accession number is 27081. Mass spectrometry data can be visualized using PRIDE Inspector [20] (http://tinyurl.com/csffalc) and Scaffold .sf3 free viewer (https://proteomecommons.org/tool.jsp?i=1009). 


\section{Analysis of secreted protein candidates}

For verification of secreted protein candidates an amino acid sequence analysis was performed with SignalP 3.0 and SecretomeP 2.0 (CBS, Technical University Copenhagen, Denmark, www. cbs.tdu.dk). SignalP 3.0 was used to verify the presence and location of signal peptide cleavage sites in amino acid sequences. SecretomeP 2.0 was applied to categorize proteins as non-classical secreted in case they are devoid of a signal peptide but obtain an NN-score above the threshold of 0.5 .

\section{Statistical analysis}

All statistical analyses were performed with the Student's T-test. Significant differences between groups are indicated when $p<0.05$.

\section{Results}

\section{RSV-induced lipolysis in human SGBS adipocytes}

To investigate the RSV-induced secretome changes of mature SGBS adipocytes, the optimal RSV concentration was determined by which the highest TG hydrolysis and the lowest apoptotic stress response were observed. This was measured by intracellular TG content and glycerol release. A significant reduction of TG was observed with RSV treatment of 50 $\mu \mathrm{M}$ and higher (Figure $1 \mathrm{~A}$ and $1 \mathrm{~B}$ ). A significant increase of glycerol release was observed with $75 \mu \mathrm{M}$ and $100 \mu \mathrm{M}$ RSV treatment (Figure 1C). Incubation with $200 \mu \mathrm{M}$ RSV showed a strong reduction of intracellular TG but this was together with a significant decrease of glycerol release which may indicate cytotoxic effects (Figure 1A, B and C). According to these results we choose a RSV concentration of $75 \mu \mathrm{M}$ which was the lowest concentration that produced a significant TG reduction and glycerol release (Figure 1). 
A

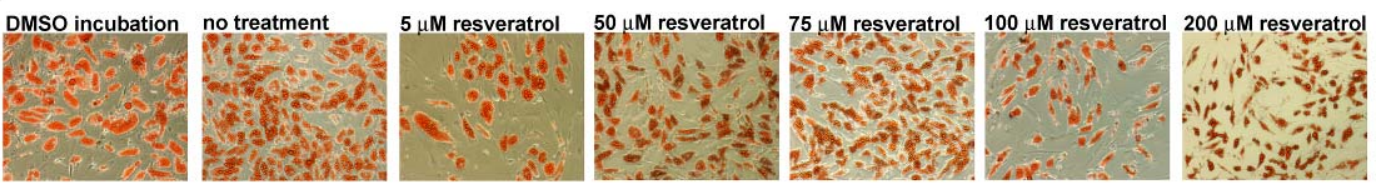

B

C
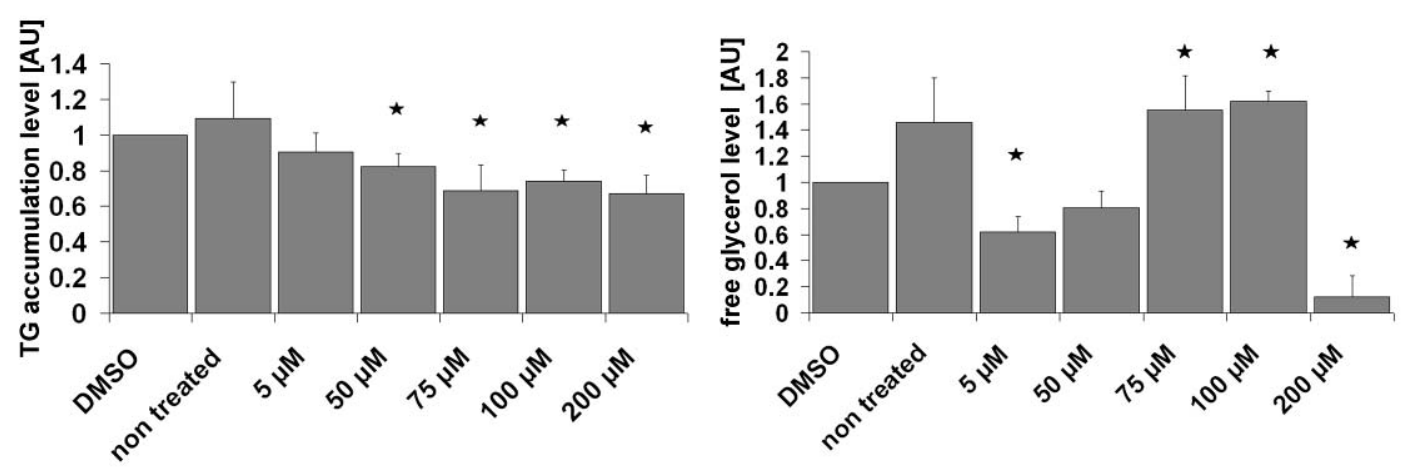

Figure 1: TG accumulation and glycerol release studies of RSV-treated SGBS adipocytes. A: Images of 13 days differentiated adipocytes which were incubated for $48 \mathrm{~h}$ with different RSV concentrations. From left to right: incubation with DMSO (control), no treatment, incubation with 5, 50, 75, 100 and $200 \mu \mathrm{M} \mathrm{RSV}$. Intracellular TG was stained by ORO and visualized by phase contrast microscopy. B: Intracellular TG accumulation corrected for cell number determined by spectrophotometry after similar conditions as in A. C: Glycerol release in the culture medium corrected for cell number determined after similar conditions as in A. Reported values are means \pm SEM of 4 independent biological replications and * indicates differences with $p<0.05$.

\section{Secretome analysis of RSV-treated adipocytes}

Changes in the secretome were determined by comparing $100 \mu \mathrm{g}$ protein samples derived from 13-day differentiated adipocytes, treated for $48 \mathrm{~h}$ with $75 \mu \mathrm{M}$ RSV or with DMSO (control). Proteins were separated by 2-DE and analyzed by PDQuest. Representative gels of the control and RSV condition, together with the master gel are shown in Figure 2. 
A

MW (kD) adipoctes untreated

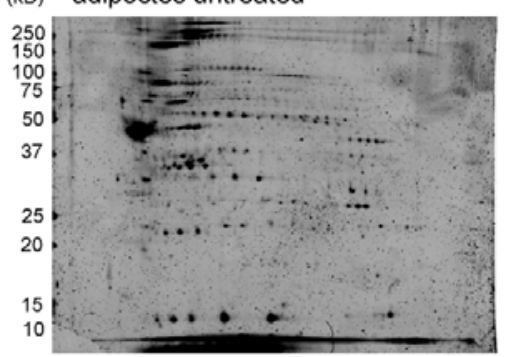

adipocytes treated with resveratrol

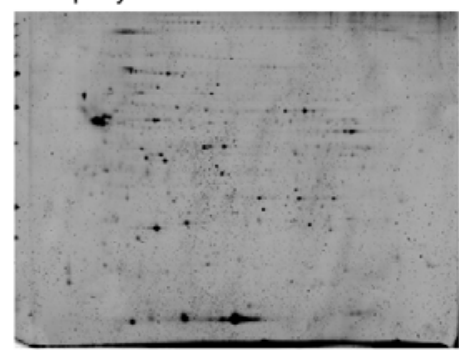

$\mathrm{B}$

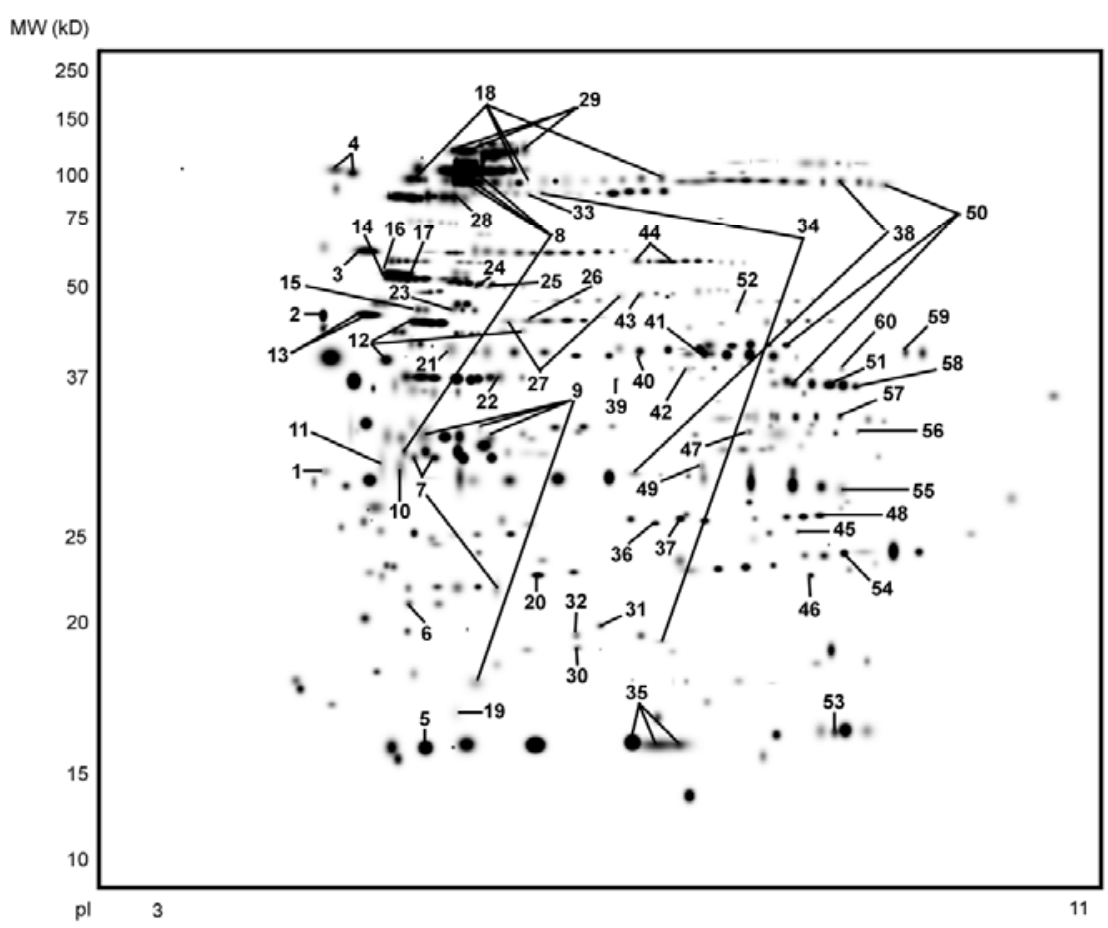

Figure 2: 2-DE gels of SGBS adipocyte secreted proteins. A: Representative gels of medium-derived secreted proteins of 13 days differentiated SGBS adipocytes in the absence (left) or presence (right) of $75 \mu \mathrm{M}$ RSV. B: Master gel with identified spots. Spot numbers refer to ID numbers in Table 1.

From a total of 484 matched spots, 155 spots were significantly $(p>0.05)$ (112) or as trend $(p>0.1)$ (43) differentially expressed. These spots were excised from the gels and analyzed by LC-ESI-MS/MS. 81 of the 155 spots were identified, which revealed 57 unique proteins. These 57 proteins were analyzed by SignalP 3.0 and Secretome 2.0 which revealed 25 proteins as classical secreted proteins, 14 proteins as non-classical secreted and 18 proteins annotated as intracellular proteins. The category of classical secreted proteins is further subcategorized into extracellular matrix (ECM) proteins, processing proteins and regulation/signaling proteins (Figure 2, Table 1, Supplement 1).

The 17 intracellular proteins are mostly associated with cell death or cell leakage. However, they were mainly identified within spots containing more than one protein and as such; they are listed in the context of spot regulation. The ECM proteins including collagens, EGF- 
containing fibulin-like extracellular matrix protein 1, fibronectin, laminin and nidogen-2 were significantly or by trend down-regulated. In addition, non-classical secreted proteins related to cellular structure components including serpin $\mathrm{H} 1$ and vimentin were significantly downregulated. Most of the processing proteins including cathepsin L1, cystatin $C$, complement factor $D$ (by trend) and procollagen $C$-endopeptidase enhancer 1 were significantly induced by RSV. Further analyses of the identified proteins revealed that RSV similarly regulated proteins associated with cell stress (putative heat shock $70 \mathrm{kDa}$ protein 7 ) and proteins involved in the regulation of apoptosis (galectin-1 and haptoglobin). Both stress- and apoptosis-related proteins were significantly as well as by trend induced by RSV (Table1). In addition, peroxiredoxin 1 (by trend), peroxiredoxin 2 (significant) and superoxide dismutase (by trend), which are proteins that protect cells from radicals, peroxides and oxidative damage, were also induced by RSV. Apolipoprotein $E(A p o E)$ and fatty acid binding protein (FABP), which are involved in TG regulation, were also significant RSV-induced. In addition, RSV mediated a down-regulation of plasminogen activator inhibitor 1 (PAl-1) (significant) and pigment epithelium-derived factor (PEDF) (by trend) and a significant up-regulation of adiponectin.

Table 1: Identification of secreted proteins from RSV-treated or non-treated adipocytes

\begin{tabular}{|c|c|c|c|c|c|c|}
\hline $\begin{array}{l}\text { Accession } \\
\text { number }\end{array}$ & \multicolumn{2}{|l|}{ Protein name } & ID & $\begin{array}{c}\mathrm{MW} \\
{[\mathrm{kDa}]}\end{array}$ & $\begin{array}{l}\text { Fold } \\
\text { change }\end{array}$ & $\begin{array}{l}\text { Treatment } \\
p \text {-value }\end{array}$ \\
\hline \multicolumn{7}{|c|}{$\begin{array}{l}\text { classical secreted } \\
\text { extracellular matrix }\end{array}$} \\
\hline P27797 & Calreticulin & & 2 & 70.3 & $3.3 E+00$ & 6.17E-02 \\
\hline \multirow[t]{8}{*}{ P02452 } & Collagen alpha-1(I) chain & C-term & 7 & 35.8 & $-1.9 \mathrm{E}+00$ & 7.35E-02 \\
\hline & & & 7 & 243.4 & $-2.1 E+05$ & 2.64E-02 \\
\hline & & & 7 & 249.1 & $-3.8 E+01$ & $5.49 \mathrm{E}-02$ \\
\hline & & & 7 & 249.2 & $-4.3 E+00$ & 4.75E-02 \\
\hline & & & 17 & 246.2 & $-2.6 E+05$ & 4.94E-06 \\
\hline & & & 17 & 249.7 & $-5.6 E+00$ & 3.29E-02 \\
\hline & & & 17 & 241.0 & $5.8 E+00$ & 7.54E-03 \\
\hline & & & 17 & 244.5 & $-2.8 \mathrm{E}+00$ & 6.33E-02 \\
\hline \multirow{7}{*}{ P02461 } & Collagen alpha-1(III) chain & C-term & 6 & 34.9 & $-9.4 E+04$ & 2.28E-05 \\
\hline & & C-term & 6 & 34.9 & $-1.7 E+00$ & 4.46E-02 \\
\hline & & C-term & 6 & 21.0 & $-3.8 E+04$ & $9.40 \mathrm{E}-07$ \\
\hline & & C-term & 7 & 35.8 & $-1.9 E+00$ & 7.35E-02 \\
\hline & & & 7 & 243.4 & $-2.1 E+05$ & 2.64E-02 \\
\hline & & & 7 & 249.1 & $-3.8 \mathrm{E}+01$ & $5.49 E-02$ \\
\hline & & & 7 & 249.2 & $-4.3 E+00$ & 4.75E-02 \\
\hline P12109 & Collagen alpha- $1(\mathrm{VI})$ chain & & 26 & 209.6 & $6.4 \mathrm{E}+00$ & $6.29 E-02$ \\
\hline P08123 & Collagen alpha-2(I) chain & & 57 & 242.1 & $-4.7 E+00$ & 1.24E-02 \\
\hline $\mathrm{P} 12110$ & Collagen alpha-2(VI) chain (isoform 2C2) & & 31 & 221.3 & $3.2 E+00$ & $9.10 \mathrm{E}-02$ \\
\hline Q12805 & $\begin{array}{l}\text { EGF-containing fibulin-like extracellular matri) } \\
\text { protein } 1 \text { (isoform 1) }\end{array}$ & & 12 & 79.1 & $-2.2 E+05$ & 7.29E-03 \\
\hline & & & 12 & 79.0 & $-7.1 \mathrm{E}+04$ & 2.45E-04 \\
\hline $\mathrm{P} 02751$ & Fibronectin & & 27 & 263.8 & $-2.8 E+04$ & 1.10E-02 \\
\hline & & & 27 & 263.2 & $-3.1 E+05$ & 6.86E-04 \\
\hline & & & 27 & 264.5 & $-1.3 E+05$ & 1.71E-02 \\
\hline P11047 & Laminin subunit gamma-1 & & 4 & 248.2 & $-1.8 E+01$ & 4.78E-02 \\
\hline & & & 4 & 250.0 & $-1.4 E+05$ & 1.54E-02 \\
\hline Q14112 & Nidogen-2 & & 3 & 128.8 & $-7.8 \mathrm{E}+00$ & $9.04 \mathrm{E}-02$ \\
\hline
\end{tabular}


Table 1: Identification of secreted proteins from RSV-treated or non-treated adipocytes

\begin{tabular}{|c|c|c|c|c|c|}
\hline $\begin{array}{c}\text { Accession } \\
\text { number }\end{array}$ & Protein name & ID & $\begin{array}{c}\mathrm{MW} \\
{[\mathrm{kDa}]}\end{array}$ & $\begin{array}{c}\text { Fold } \\
\text { change }\end{array}$ & $\begin{array}{c}\text { Treatment } \\
p \text {-value }\end{array}$ \\
\hline \multicolumn{6}{|c|}{ classical secreted } \\
\hline P08253 & 72 kDa type IV collagenase & 13 & 100.0 & $-1.4 \mathrm{E}+00$ & 3.45E-02 \\
\hline P07711 & Cathepsin L1 & 53 & 38.9 & $6.8 E+00$ & 2.49E-02 \\
\hline A6XNE2 & Complement factor D preproprotein & 45 & 27.6 & $3.6 \mathrm{E}+00$ & 7.86E-02 \\
\hline P01034 & Cystatin-C & 50 & 13.7 & $5.1 E+00$ & 7.16E-03 \\
\hline P01033 & Metalloproteinase inhibitor 1 & 52 & 30.6 & $-3.6 E+04$ & 2.72E-02 \\
\hline Q15113 & Procollagen C-endopeptidase enhancer 1 & 48 & 47.1 & $2.3 \mathrm{E}+00$ & 4.65E-02 \\
\hline \multicolumn{6}{|c|}{ regulation/signaling } \\
\hline P11021 & $78 \mathrm{kDa}$ glucose-regulated protein, HSPA5 protein & $\begin{array}{l}15 \\
16\end{array}$ & $\begin{array}{l}105.5 \\
104.3\end{array}$ & $\begin{array}{c}-1.1 \mathrm{E}+05 \\
2.4 \mathrm{E}+00\end{array}$ & $\begin{array}{l}\text { 1.15E-02 } \\
6.44 \mathrm{E}-02\end{array}$ \\
\hline Q15848 & Adiponectin & $\begin{array}{c}9 \\
10\end{array}$ & $\begin{array}{l}33.4 \\
33.9\end{array}$ & $\begin{array}{c}\mathbf{2 . 3 E}+00 \\
-1.4 \mathrm{E}+00\end{array}$ & $\begin{array}{l}3.09 \mathrm{E}-02 \\
8.52 \mathrm{E}-02\end{array}$ \\
\hline P02649 & Apolipoprotein E & $\begin{array}{c}8 \\
8 \\
8 \\
8 \\
23\end{array}$ & \begin{tabular}{l|l|}
38.2 & \\
17.6 \\
37.8 \\
39.3 \\
91.1
\end{tabular} & $\begin{array}{c}2.8 E+00 \\
9.3 E+00 \\
-9.7 E+04 \\
1.8 E+00 \\
-1.6 E+04\end{array}$ & $\begin{array}{l}6.77 \mathrm{E}-02 \\
1.50 \mathrm{E}-02 \\
4.81 \mathrm{E}-04 \\
6.60 \mathrm{E}-03 \\
7.28 \mathrm{E}-03\end{array}$ \\
\hline P09382 & Galectin-1 & 5 & 12.7 & $2.3 \mathrm{E}+00$ & $6.51 \mathrm{E}-02$ \\
\hline P00738 & Haptoglobin & 30 & 19.3 & $7.3 E+00$ & 4.54E-02 \\
\hline Q02818 & Nucleobindin-1 & 14 & 74.4 & $-4.4 E+00$ & 3.31E-03 \\
\hline \multirow[t]{2}{*}{ P36955 } & Pigment epithelium-derived factor & 20 & 51.4 & $-6.0 \mathrm{E}+00$ & $6.02 \mathrm{E}-02$ \\
\hline & & 33 & 19.1 & $-1.6 \mathrm{E}+04$ & 5.89E-02 \\
\hline P05121 & Plasminogen activator inhibitor 1 & 38 & 49.8 & $-2.9 E+00$ & 1.42E-03 \\
\hline P30101 & Protein disulfide-isomerase A3 & 25 & 66.9 & $-6.6 \mathrm{E}+00$ & 7.80E-02 \\
\hline \multirow[t]{2}{*}{ P02787 } & Serotransferrin & 41 & 116.6 & $-1.9 E+01$ & 3.84E-05 \\
\hline & & 41 & 116.4 & $-2.9 \mathrm{E}+00$ & 8.53E-02 \\
\hline \multicolumn{6}{|c|}{ non-classical secreted } \\
\hline P06733 & Alpha-enolase (Isoform alpha-enolase) & 48 & 47.1 & $2.3 E+00$ & 4.65E-02 \\
\hline P09496 & Clathrin light chain $A$ * & 1 & 32.9 & $4.3 \mathrm{E}+00$ & 7.93E-02 \\
\hline Q13011 & Delta(3,5)-Delta(2,4)-dienoyl-CoA isomerase & 46 & 33.6 & 4.1E+00 & 9.36E-03 \\
\hline P09622 & Dihydrolipoyl dehydrogenase & 49 & 72.6 & $2.5 \mathrm{E}+00$ & $9.41 \mathrm{E}-02$ \\
\hline \multirow{4}{*}{ P15090 } & Fatty acid-binding protein, adipocyte & 32 & 12.9 & $1.4 \mathrm{E}+02$ & 3.58E-02 \\
\hline & & 32 & 12.9 & $8.8 E+01$ & 3.36E-02 \\
\hline & & 32 & 13.0 & $3.4 \mathrm{E}+00$ & 4.54E-02 \\
\hline & & 35 & 27.2 & $5.0 \mathrm{E}+00$ & 3.03E-02 \\
\hline P00338 & L-lactate dehydrogenase A chain & 53 & 38.9 & $6.8 E+00$ & 2.49E-02 \\
\hline P07195 & L-lactate dehydrogenase $B$ chain & 37 & 49.7 & $-1.4 \mathrm{E}+00$ & $6.62 \mathrm{E}-02$ \\
\hline Q06830 & Peroxiredoxin-1 & 51 & 23.6 & $2.6 E+00$ & 8.94E-02 \\
\hline P32119 & Peroxiredoxin-2 & 19 & 21.5 & 4.1E+00 & 1.83E-02 \\
\hline P30086 & Phosphatidylethanolamine-binding protein 1 & 43 & 21.5 & $5.4 \mathrm{E}+00$ & 9.55E-03 \\
\hline P48741 & Putative heat shock $70 \mathrm{kDa}$ protein $7^{*}$ & 16 & 104.3 & $2.4 \mathrm{E}+00$ & $6.44 \mathrm{E}-02$ \\
\hline P50454 & Serpin H1 & 56 & 50.0 & $-1.1 \mathrm{E}+02$ & 4.55E-05 \\
\hline P00441 & Superoxide dismutase [Cu-Zn] & 28 & 18.9 & $3.3 E+00$ & $7.88 \mathrm{E}-02$ \\
\hline \multirow[t]{3}{*}{ P08670 } & Vimentin & 11 & 49.4 & $-3.4 \mathrm{E}+00$ & 7.78E-02 \\
\hline & & 11 & 66.5 & $-1.4 \mathrm{E}+01$ & 5.78E-03 \\
\hline & & 11 & 61.2 & $-1.2 E+04$ & 3.66E-08 \\
\hline \multicolumn{6}{|c|}{ intracellular } \\
\hline P10809 & $60 \mathrm{kDa}$ heat shock protein & 22 & 74.5 & $-2.7 E+00$ & 4.98E-02 \\
\hline Q03154 & Aminoacylase-1 & 36 & 47.9 & $2.7 E+00$ & 9.53E-02 \\
\hline \multirow[t]{2}{*}{ P60709 } & Actin & 21 & 48.1 & $-2.5 \mathrm{E}+00$ & 7.36E-02 \\
\hline & & 39 & 48.7 & $-7.6 \mathrm{E}+00$ & 3.19E-05 \\
\hline Q9UKK9 & ADP-sugar pyrophosphatase & 10 & 33.9 & $-1.4 \mathrm{E}+00$ & $8.52 \mathrm{E}-02$ \\
\hline P00325 & Alcohol dehydrogenase 1B & 55 & 46.8 & 4.1E+00 & 4.57E-02 \\
\hline $\mathrm{P} 12277$ & Creatine kinase B-type & 21 & 48.1 & $-2.5 \mathrm{E}+00$ & 7.36E-02 \\
\hline Q16555 & Dihydropyrimidinase-related protein 2 & 40 & 85.7 & $-2.7 E+00$ & 4.60E-02 \\
\hline Q13561 & Dynactin subunit 2 & 20 & 51.4 & $-6.0 \mathrm{E}+00$ & $6.02 \mathrm{E}-02$ \\
\hline \multirow[t]{4}{*}{ P04075 } & Fructose-bisphosphate aldolase A & 47 & 53.3 & $3.4 \mathrm{E}+00$ & 9.96E-02 \\
\hline & & 47 & 47.2 & $4.9 \mathrm{E}+00$ & 7.99E-02 \\
\hline & & 47 & 240.0 & $-2.1 \mathrm{E}+00$ & 7.09E-02 \\
\hline & & 55 & 46.8 & 4.1E+00 & 4.57E-02 \\
\hline
\end{tabular}


Table 1: Identification of secreted proteins from RSV-treated or non-treated adipocytes

\begin{tabular}{|c|c|c|c|c|c|}
\hline $\begin{array}{l}\text { Accession } \\
\text { number }\end{array}$ & Protein name & ID & $\begin{array}{c}\mathrm{MW} \\
{[\mathrm{kDa}]}\end{array}$ & $\begin{array}{c}\text { Fold } \\
\text { change }\end{array}$ & $\begin{array}{l}\text { Treatment } \\
p \text {-value }\end{array}$ \\
\hline \multicolumn{6}{|c|}{ intracellular } \\
\hline P04406 & Glyceraldehyde-3-phosphate dehydrogenase & 54 & 41.3 & $7.9 \mathrm{E}+00$ & 5.79E-02 \\
\hline P08107 & Heat shock $70 \mathrm{kDa}$ protein $1 \mathrm{~A} / 1 \mathrm{~B}$ & $\begin{array}{l}23 \\
24\end{array}$ & $\begin{array}{l}91.1 \\
91.4\end{array}$ & $\begin{array}{l}-1.6 \mathrm{E}+04 \\
-1.9 \mathrm{E}+04\end{array}$ & $\begin{array}{l}7.28 \mathrm{E}-03 \\
1.72 \mathrm{E}-03\end{array}$ \\
\hline P22626 & $\begin{array}{l}\text { Heterogeneous nuclear ribonucleoproteins } \\
\text { A2/B1(Isoform B1) }\end{array}$ & 42 & 25.8 & $3.6 \mathrm{E}+00$ & 2.85E-02 \\
\hline P40925 & Malate dehydrogenase & 44 & 38.8 & $3.1 \mathrm{E}+00$ & 9.36E-02 \\
\hline P15531 & Nucleoside diphosphate kinase A & 29 & 19.6 & $2.0 \mathrm{E}+00$ & $9.42 \mathrm{E}-02$ \\
\hline P30041 & Peroxiredoxin-6 & 35 & 27.2 & $5.0 \mathrm{E}+00$ & 3.03E-02 \\
\hline P07737 & Profilin-1 & 50 & 13.7 & $5.1 \mathrm{E}+00$ & 7.16E-03 \\
\hline P60174 & Triosephosphate isomerase & 34 & 26.7 & $-6.5 E+00$ & 4.84E-03 \\
\hline & & 34 & 27.2 & $9.5 \mathrm{E}+00$ & 3.13E-02 \\
\hline O75347 & Tubulin-specific chaperone A & 18 & 9.8 & $3.5 \mathrm{E}+00$ & 5.14E-02 \\
\hline
\end{tabular}

Table 1: Identification of secreted proteins from RSV-treated or non-treated SGBS adipocytes by 2-DE followed by LC-MS/MS. Proteins with the identical ID number were identified in the same spot and proteins marked with * were identified as novel adipocyte secreted proteins. Bold lines mark proteins/protein isoforms which are significantly differentially expressed $(p<0.05)$. Thin lines relate to a trend for change $(0.05<p<0.10)$.

\section{Validation of the identified proteins}

Comparison of the RSV data set with recent human and rodent adipokine proteomics studies [14, 21-30] identified clathrin light chain $A$ and putative heat shock $70 \mathrm{kDa}$ protein 7 as novel adipocyte secreted proteins. Most of the identified differentially expressed proteins are annotated as classical adipocyte secreted proteins. Since other proteomics studies on RSVinduced secretome changes are lacking we compared our data with currently existing gene and protein expression data with respect to RSV-specific effects on mouse 3T3-L1 adipocytes [31-34], visceral adipose tissue from Zucker rats [35], human SGBS adipocytes $[36,37]$, human adipose tissue explants [6] and human visceral adipocytes [38]. Within these data sets interleukin (IL) $1 \beta, 6,8$, monocyte chemotactic protein (MCP)-1, tumor necrosis factor (TNF)- $\alpha$, PAl-1, peroxisome proliferator-activated receptor (PPAR)- $\gamma$, resistin, Sirt1 and visfatin were found down-regulated by RSV. Only adiponectin and once PPARy were found to be RSV-induced [6, 31-33, 35-39]. Comparison with our data confirmed only adiponectin and PAI-1 expression and regulation [6, 31, 33, 35, 38].

\section{Discussion}

Obesity is associated with the development of metabolic diseases and increased mortality. Consequently, obesity has a considerable impact on personal life quality $[13,40]$. RSV is known to mimic beneficial effects of calorie restriction and may positively affect obesityassociated diseases $[1,3,5]$. Our study is the first using a proteomic approach to study the effect of RSV on the secretome of human adipocytes. The use of human SGBS adipocytes was based on the fact that human fat biopsies are limited with respect to the amount of preadipocytes and their differentiation capacity. In contrast, human preadipocytes from subcutaneous adipose tissue of an infant with Simson-Golabi-Behmel syndrome (SGBS) can 
display a differentiation capacity of up to $90 \%$ and retain this capacity over at least 30 generations while being morphologically, biochemically and functionally similar to primary preadipocytes [15]. In addition, Fischer-Posovszky et al. [41], our group [14] and Luo et al. [42] showed that SGBS cell are an ideal cell model to study adipocyte biology and its secretion behavior. As such, SGBS (pre)adipocytes are a good choice to investigate the adipocyte differentiation, manipulation and secretome. Our results show that RSV, concomitantly with reduced intracellular TG levels, positively affected the SGBS adipocyte secretion profile with respect to the obesity-associated metabolic pattern. Furthermore, two novel adipocyte-secreted proteins were discovered from which the expression was regulated by RSV.

RSV-treated adipocytes showed a significant decrease in intracellular lipid (TG) content which might be the result of increased intracellular lipolysis, as supported by an increased basal glycerol release. The intracellular lipolysis is mediated by lipase activity. As such, HSL and ATGL lipases are responsible for more than $95 \%$ of the lipase activity in human adipocytes [43]. Rayalam et al. [32] showed in 3T3-L1 adipocytes a RSV-induced SIRT1 repression of peroxisome proliferator-activated receptor gamma (PPARY), CCAAT/enhancer binding protein $\alpha(\mathrm{C} / \mathrm{EBP} \alpha)$, FAS and lipoprotein lipase (LPL), which is in line with inhibition of lipid accumulation in adipocytes. In addition, Lasa et al. [44] demonstrated clearly a RSVinduced lipolysis by ATGL activity in SGBS and 3T3-L1. Rayalam et al. [32] further showed a RSV-induced reduction of the HSL expression, which we also observed in the SGBS cells (Supplement 2).

The used RSV concentration of $75 \mu \mathrm{M}$ is high compared to physiological RSV concentrations. Such high concentrations are not achieved by normal diets and also not measured in the blood plasma $[1,5,45]$. In addition, the bioavailability of RSV in in vivo studies is dramatically reduced due to a rapid and extensive conversion to resveratrol glucuronides and resveratrol sulfates [46]. Therefore, pharmacological intervention with high RSV concentrations might be needed to bring about the RSV-induced beneficial effects. However, Timmers et al. demonstrated at physiological RSV concentration beneficial effects on insulin sensitivity and improved blood glucose, triglycerides and cytokines levels [5]. Such in vivo studies are based on longer intervention periods [5]. In contrast, in vitro studies which mostly use cell models, have to achieve the same effects in a shorter intervention period until $96 \mathrm{~h}$. This limitation requires an increased RSV concentration which was used here over a period of $48 \mathrm{~h}$.

$\mathrm{RSV}$ of in vitro experiments was demonstrated to be oxidized after $24 \mathrm{~h}$ which decreases its activity and generates oxidative stress by producing $\mathrm{H}_{2} \mathrm{O}_{2}$ [47]. Increased $\mathrm{H}_{2} \mathrm{O}_{2}$ levels were shown to decrease SIRT1 expression which might negatively effects the SIRT1-induced lipolysis and inhibition of lipid accumulation in adipocytes [32]. Oxidized RSV could also be the explanation of the significant decrease of the glycerol release of $5 \mu \mathrm{M}$ RSV treated 


\section{CHAPTER 6}

adipocytes in our study. To avoid the oxidation of RSV and in turn increased levels of oxidative stress, RSV might be protected by adding vitamin C or superoxide dismutase (SOD) to the culture medium. On the other hand, additional substances might lead to crossreaction and a differentially expressed secretome profile. In addition, vitamin C and SOD are shown to even enhance the $\mathrm{H}_{2} \mathrm{O}_{2}$ production [48] or produce inconsistent results [49]. Therefore, no extra measures were taken to protect the RSV in the medium. To investigate the presence of functional RSV, we performed SIRT1 Western blotting of $48 \mathrm{~h} 75 \mu \mathrm{M}$ RSVtreated or non-treated SGBS adipocytes which revealed a RSV-induced significant increase of SIRT1 expression (Supplement 2). SIRT1 is thought to be indirectly activated by RSV via Epac1 and AMPK signaling pathway [10] and is down-regulated by $\mathrm{H}_{2} \mathrm{O}_{2}$ production [50-52]. Apparently, functional RSV was present to explain the measured effects including increased lipolysis, decreased TG content and secretome changes, although we cannot totally exclude $\mathrm{H}_{2} \mathrm{O}_{2}$-induced secretome affections due to oxidized RSV.

The observed RSV-induced reduction in intracellular TG content might result in a decreased adipocyte cell size and induces structural remodeling $[53,54]$. In line, we observed that most of structural proteins including ECM components, serpin $\mathrm{H} 1$ as a collagen binding protein and filament-related proteins (vimentin) are significantly down-regulated while processing proteins including cathepsin L1, cystatin-C and procollagen C-endopeptidase enhancer 1 are significantly up-regulated after RSV treatment. It has been shown that reduced expression levels of vimentin results in a decreased lipid droplet formation [55]. As such, this may explain the RSV-mediated reduction in vimentin secretion which is accompanied by decreased lipid content. Reduced cell size urges a decreased expression of cell structure proteins and complies with the lower relative abundance of ECM proteins and the upregulation of the processing proteins allowing the remodeling of the ECM. Cell shrinking may lead to cellular stress and may eventually induce apoptosis [53, 54]. Indeed, Rayalam et al. [32] reported increased apoptosis in RSV-treated 3T3-L1 adipocytes. In addition, Mader et al. [51] investigated a SIRT1-independent way of RSV-induced apoptosis in SGBS adipocytes. It was shown that RSV inhibited phosphoinositide 3 kinase-driven phosphorylation of Akt that in turn induces Bax activation. This resulted in caspase-dependent apoptosis. The present study identified apoptosis and stress-related proteins including heat shock proteins and galectin-1 [56], which were up-regulated by RSV. It confirms the occurrence of cellular stress and increases the liability towards apoptosis, mimicking the effect of calorie restriction on adipocytes [51]. Although we cannot conclude whether RSV mediates apoptosis in fat cells via cell shrinking or via a caspase-dependent pathway, our findings support the theory of RSV-induced adipocyte apoptosis. This may have implications for a potential application of RSV to reduce fat content in obese subjects by mimicking calorie restriction and adipocyte apoptosis. 
Beside the induction of stress- and apoptosis-related proteins we observed that RSV also induced proteins that protect cells from oxidative damage like peroxiredoxin 1, 2 and superoxide dismutase. It has been show previously that RSV treatment activates antioxidants and in turn reduces mitochondrial reactive oxygen species in human endothelial cells [57]. Peroxiredoxin (Prx) proteins are generally known to protect against oxidative stress. Prx-2 reduces generated hydrogen peroxide non-catalytically [58] while the redox function of Prx-1 is still under discussion. Kim et al. [59] demonstrated that Prx-1 is negatively correlated with the apoptosis signaling-regulating kinase 1 (ASK1) that stimulates apoptosis. On the other hand, Morinaka et al. [60] showed that high levels of oxidative stress induced the formation of oligomeric Prx-1 which leads to p53-induced cell apoptosis. Upregulation of Prx-proteins suggests higher levels of oxidative stress which may be induced by apoptosis or increased lipolysis and up-regulated metabolic enzymes [61]. It shows that RSV-treated adipocytes react in a cell survival manner to deal with increased cellular stress.

Next to the apoptosis regulation and the anti-oxidative capacity of RSV on adipocytes we identified in this study two novel adipocyte-secreted proteins. They were classified as nonclassical secreted proteins. First, clathrin light chain $A$ is a protein of the coated pits and vesicles. As such, it is involved in accumulation of proteins on the plasma membrane like glucose transporter (GLUT) 4 in adipocytes [62]. Translocation of GLUT 4 proteins is an important regulatory mechanism of the glucose transport into the cells (adipocytes) and may play an important role in the regulation of blood circulating glucose levels. Second, the putative heat shock $70 \mathrm{kDa}$ protein 7 belongs to the heat shock protein (HSP) 70 family and as such mostly associated with the stress response. Not much is known about the putative heat shock $70 \mathrm{kDa}$ protein 7 . However, HSP70 chaperons are ubiquitous proteins and mostly associated with adenosine triphosphate-dependent biological processes including protein folding, degradation and translocation [63].

RSV mimics the effect of calorie restriction, which includes a less inflammatory phenotype as well as an improvement of metabolic complications [5, 64]. The observed regulation patterns of adiponectin, ApoE, PAI-1 and PEDF indicate that RSV reverses the human adipocyte secretion profile towards a less inflammatory phenotype and a more insulin-sensitizing pattern. Adiponectin, ApoE, PAI-1 and PEDF are known to be involved in inflammatory processes [65-70]. Therefore, a reduced secretion of PAI-1 and PEDF and elevated secretion of the anti-inflammatory factors adiponectin and ApoE by RSV might indicate a potential anti-inflammatory effect of RSV. ApoE, which is known to regulate TG turnover as well as expression of genes which are involved in lipid synthesis to fulfill the energy and lipid homeostasis of cells, induces a conversion of pro-inflammatory M1 macrophages towards M2 macrophages with anti-inflammatory phenotypes [67]. This indicates that RSV-induced ApoE secretion might be involved in the switch between macrophage phenotypes, 


\section{CHAPTER 6}

contributing to its anti-inflammatory effect. To the best of our knowledge this is the first report that shows a RSV-mediated induction of ApoE secretion from human adipocytes. However, increase of ApoE secretion might also be a protection mechanism against oxidative stress which is shown by Tarnus et al. [71]. Beside a possible $\mathrm{H}_{2} \mathrm{O}_{2}$ environment due to oxidized RSV, a RSV- and as such a SIRT1-induced lipolysis and in turn cell shrinking processes also cause oxidative stress [32, 51, 53, 54]. In contrast to the finding of Tarnus et al. [71], Esperitu et al. [72] clearly demonstrated in a $\mathrm{H}_{2} \mathrm{O}_{2}$ environment an oxidative stress-stimulated Apo-E suppression in mice adipocytes. In addition, the here observed regulation pattern of adiponectin and PAI-1 are contrary to the protein regulation pattern under $\mathrm{H}_{2} \mathrm{O}_{2}$-induced oxidative stress $[73,74]$. Therefore, we conclude that the Apo-E up-regulation and its antiinflammatory actions are the result of functional RSV and its downstream signaling.

Together with the increased ApoE secretion, the RSV-induced up-regulation of adiponectin and down-regulation of PEDF (by trend) and PAI-1 indicates an adipocyte secretome profile towards a more insulin-sensitizing pattern. Adiponectin is involved in glucose regulation, fatty acid catabolism and increased insulin sensitivity. The levels of circulating adiponectin are reduced during the development of obesity, which negatively influences insulin sensitivity and in turn may lead to metabolic syndrome complications [75]. The observed up-regulation of adiponectin secretion might be a beneficial effect with respect to increased glucose uptake in muscles, insulin sensitivity and suppressed gluconeogenesis in the hepatocytes [76]. In addition, adiponectin is an anti-inflammatory factor that inhibits pro-inflammatory factors including TNF- $\alpha$ and reactive oxygen species [66]. The observed RSV-induced adiponectin levels are in line with recent literature [6, 31, 33-35, 38] although Derdemezis et al. [36] showed no RSV-stimulated adiponectin expression in SGBS adipocytes. This result was explained by the lower inflammatory characteristics of SGBS adipocyte type compared to visceral adipocytes [36]. However, the use of only $10 \mu \mathrm{M}$ or $25 \mu \mathrm{M}$ RSV concentration over a period of $24 \mathrm{~h}$ and $48 \mathrm{~h}$ [36] might be the more reasonable explanation for the absence of RSV-stimulated effects which is also shown in our RSV concentration course study.

PEDF is associated with enlarged adipose tissue mass and insulin resistance [77, 78]. Down-regulation of PEDF may be beneficial for insulin sensitivity and decreases the inflammatory phenotype within obese subjects [65]. Here all identified PEDF spots were down-regulated by trend which shows a potential regulation mechanism of PEDF during RSV treatment. As such, it is to the best of our knowledge the first report that shows a RSVmediated down-regulation of PEDF secretion from human adipocytes.

PAl-1 stimulates the fibrinogen formation by inhibiting matrix metalloproteinases and fibrinolysis. Such increased fibrinogen expression is also related to low-grade inflammation [70]. As such, a down-regulation of PAI-1 may decrease the chance of development of obesity-induced thrombosis and cardiovascular diseases [70, 79]. The RSV-mediated down- 


\section{SECRETOME OF RSV-TREATED ADIPOCYTES}

regulation of PAl-1 found in the present study is in-line with literature and might contribute to the potential anti-inflammatory action of RSV $[6,31,34]$.

\section{Conclusion}

We showed caloric-restrictive effects of RSV in human adipocytes and in turn a changed adipocyte secretion profile that indicates ECM remodeling, reduced cellular stress and modification of apoptosis sensitivity. In addition, a RSV-induced up-regulation of ApoE and adiponectin together with a down-regulation of PAI-1 and PEDF may change the adipocyte secretion profile towards a more beneficial pattern that may improve the obesity-associated pro-inflammatory state, insulin sensitivity and thrombotic phenotype.

\section{Acknowledgement}

We thank Freek Bouwman of the Department Human Biology, Maastricht University, The Netherlands, Eric Royackers of the Biomedical Research Institute, Hasselt University, Belgium for their practical support and Prof. Dr. Martin Wabitsch of the Endocrine Research Laboratory, Division of Pediatric Endocrinology and Diabetes, Ulm University Medical Center, Germany for the kind gift of SGBS cells.

\section{References}

1. Baur, J.A., et al., Resveratrol improves health and survival of mice on a high-calorie diet. Nature, 2006. 444(7117): p. 337-42.

2. Roemer, K. and M. Mahyar-Roemer, The basis for the chemopreventive action of resveratrol. Drugs Today (Barc), 2002. 38(8): p. 571-80.

3. Baur, J.A., Resveratrol, sirtuins, and the promise of a DR mimetic, in Mech Ageing Dev. 2010. p. 261-9.

4. Pearson, K.J., et al., Resveratrol delays age-related deterioration and mimics transcriptional aspects of dietary restriction without extending life span. Cell Metab, 2008. 8(2): p. 157-68.

5. Timmers, S., et al., Calorie Restriction-like Effects of 30 Days of Resveratrol Supplementation on Energy Metabolism and Metabolic Profile in Obese Humans. Cell Metab, 2011. 14(5): p. 612-22. 
6. Olholm, J., et al., Anti-inflammatory effect of resveratrol on adipokine expression and secretion in human adipose tissue explants. Int J Obes (Lond), 2010. 34(10): p. 154653.

7. Howitz, K.T., et al., Small molecule activators of sirtuins extend Saccharomyces cerevisiae lifespan. Nature, 2003. 425(6954): p. 191-6.

8. Lin, S.J., P.A. Defossez, and L. Guarente, Requirement of NAD and SIR2 for lifespan extension by calorie restriction in Saccharomyces cerevisiae. Science, 2000. 289(5487): p. 2126-8.

9. Park, C.E., et al., Resveratrol stimulates glucose transport in $\mathrm{C} 2 \mathrm{C} 12$ myotubes by activating AMP-activated protein kinase. Exp Mol Med, 2007. 39(2): p. 222-9.

10. Park, S.J., et al., Resveratrol ameliorates aging-related metabolic phenotypes by inhibiting cAMP phosphodiesterases. Cell, 2012. 148(3): p. 421-33.

11. Gregoire, F.M., Adipocyte differentiation: from fibroblast to endocrine cell. Exp Biol Med (Maywood), 2001. 226(11): p. 997-1002.

12. Lehr, S., S. Hartwig, and H. Sell, Adipokines: A treasure trove for the discovery of biomarkers for metabolic disorders. Proteomics Clin Appl, 2012. 6(1-2): p. 91-101.

13. Diamanti-Kandarakis, E., et al., The Impact of Endocrine Disruptors on Endocrine Targets. Horm Metab Res, 2010. 42(8): p. 543-52.

14. Rosenow, A., et al., Identification of Novel Human Adipocyte Secreted Proteins by Using SGBS Cells. J Proteome Res, 2010. 9: p. 5389-401.

15. Wabitsch, M., et al., Characterization of a human preadipocyte cell strain with high capacity for adipose differentiation. Int J Obes Relat Metab Disord, 2001. 25(1): p. 815.

16. Bouwman, F., J. Renes, and E. Mariman, A combination of protein profiling and isotopomer analysis using matrix-assisted laser desorption/ionization-time of flight mass spectrometry reveals an active metabolism of the extracellular matrix of 3T3-L1 adipocytes. Proteomics, 2004. 4(12): p. 3855-63.

17. Dumont, D., et al., Characterization of mature rat oligodendrocytes: a proteomic approach. J Neurochem, 2007. 102(2): p. 562-76.

18. Barsnes, $\mathrm{H}_{\text {., }}$ et al., PRIDE Converter: making proteomics data-sharing easy. Nat Biotechnol, 2009. 27(7): p. 598-9.

19. Vizcaino, J.A., et al., The Proteomics Identifications database: 2010 update. Nucleic Acids Res, 2010. 38(Database issue): p. D736-42.

20. Wang, R., et al., PRIDE Inspector: a tool to visualize and validate MS proteomics data. Nat Biotechnol, 2012. 30(2): p. 135-7.

21. Lehr, S., et al., Identification and validation of novel adipokines released from primary human adipocytes. Mol Cell Proteomics, 2012. 11(1): p. M111 010504. 
22. Alvarez-Llamas, G., et al., Characterization of the human visceral adipose tissue secretome. Mol Cell Proteomics, 2007. 6(4): p. 589-600.

23. Chen, X., et al., Quantitative proteomic analysis of the secretory proteins from rat adipose cells using a 2D liquid chromatography-MS/MS approach. J Proteome Res, 2005. 4(2): p. 570-7.

24. Kim, J., et al., Comparative analysis of the secretory proteome of human adipose stromal vascular fraction cells during adipogenesis. Proteomics, 2010. 10(3): p. 394405.

25. Molina, $\mathrm{H}_{\text {., et }}$ al., Temporal profiling of the adipocyte proteome during differentiation using a five-plex SILAC based strategy. J Proteome Res, 2009. 8(1): p. 48-58.

26. Zhong, J., et al., Temporal profiling of the secretome during adipogenesis in humans. J Proteome Res, 2010. 9(10): p. 5228-38.

27. Zvonic, S., et al., Secretome of primary cultures of human adipose-derived stem cells: modulation of serpins by adipogenesis. Mol Cell Proteomics, 2007. 6(1): p. 18-28.

28. Chiellini, C., et al., Characterization of human mesenchymal stem cell secretome at early steps of adipocyte and osteoblast differentiation. BMC Mol Biol, 2008. 9: p. 26.

29. Lim, J.M., et al., Defining the regulated secreted proteome of rodent adipocytes upon the induction of insulin resistance. J Proteome Res, 2008. 7(3): p. 1251-63.

30. Roelofsen, $H_{\text {. }}$, et al., Comparison of isotope-labeled amino acid incorporation rates (CILAIR) provides a quantitative method to study tissue secretomes. Mol Cell Proteomics, 2009. 8(2): p. 316-24.

31. Ahn, J., et al., Resveratrol inhibits TNF-alpha-induced changes of adipokines in 3T3L1 adipocytes. Biochem Biophys Res Commun, 2007. 364(4): p. 972-7.

32. Rayalam, S., et al., Resveratrol induces apoptosis and inhibits adipogenesis in 3T3L1 adipocytes. Phytother Res, 2008. 22(10): p. 1367-71.

33. Kang, L., et al., Resveratrol modulates adipokine expression and improves insulin sensitivity in adipocytes: relative to inhibition of inflammatory responses. Biochimie, 2010. 92(7): p. 786-96.

34. Yen, G.C., et al., Effects of polyphenolic compounds on tumor necrosis factor-alpha (TNF-alpha)-induced changes of adipokines and oxidative stress in 3T3-L1 adipocytes. J Agric Food Chem, 2011. 59(2): p. 546-51.

35. Rivera, L., et al., Long-term resveratrol administration reduces metabolic disturbances and lowers blood pressure in obese Zucker rats. Biochem Pharmacol, 2009. 77(6): p. 1053-63.

36. Derdemezis, C.S., et al., Effect of Plant Polyphenols on Adipokine Secretion from Human SGBS Adipocytes. Biochem Res Int, 2011. 2011: p. 285618. 
37. Fischer-Posovszky, P., et al., Resveratrol regulates human adipocyte number and function in a Sirt1-dependent manner. Am J Clin Nutr, 2010. 92(1): p. 5-15.

38. Costa Cdos, S., et al., Resveratrol upregulated SIRT1, FOXO1, and adiponectin and downregulated PPARgamma1-3 mRNA expression in human visceral adipocytes. Obes Surg, 2011. 21(3): p. 356-61.

39. Ahn, J., et al., Dietary resveratrol alters lipid metabolism-related gene expression of mice on an atherogenic diet. J Hepatol, 2008. 49(6): p. 1019-28.

40. Dixon, J.B., The effect of obesity on health outcomes. Mol Cell Endocrinol, 2010. 316(2): p. 104-8.

41. Fischer-Posovszky, P., et al., Human SGBS Cells - a Unique Tool for Studies of Human Fat Cell Biology. Obes Facts, 2008. 1(4): p. 184-189.

42. Luo, X., et al., Identification of BMP and activin membrane-bound inhibitor (BAMBI) as a potent negative regulator of adipogenesis and modulator of autocrine/paracrine adipogenic factors. Diabetes, 2012. 61(1): p. 124-36.

43. Schweiger, M., et al., Adipose triglyceride lipase and hormone-sensitive lipase are the major enzymes in adipose tissue triacylglycerol catabolism. J Biol Chem, 2006. 281(52): p. 40236-41.

44. Lasa, A., et al., Resveratrol regulates lipolysis via adipose triglyceride lipase. J Nutr Biochem, 2011.

45. Lagouge, M., et al., Resveratrol improves mitochondrial function and protects against metabolic disease by activating SIRT1 and PGC-1alpha. Cell, 2006. 127(6): p. 110922.

46. Wenzel, E. and V. Somoza, Metabolism and bioavailability of trans-resveratrol. Mol Nutr Food Res, 2005. 49(5): p. 472-81.

47. Yang, N.C., C.H. Lee, and T.Y. Song, Evaluation of resveratrol oxidation in vitro and the crucial role of bicarbonate ions. Biosci Biotechnol Biochem, 2010. 74(1): p. 63-8.

48. Wee, L.M., et al., Factors affecting the ascorbate- and phenolic-dependent generation of hydrogen peroxide in Dulbecco's Modified Eagles Medium. Free Radic Res, 2003. 37(10): p. 1123-30.

49. Hou, Z., et al., Mechanism of action of (-)-epigallocatechin-3-gallate: auto-oxidationdependent inactivation of epidermal growth factor receptor and direct effects on growth inhibition in human esophageal cancer KYSE 150 cells. Cancer Res, 2005. 65(17): p. 8049-56.

50. Bai, S.W., et al., Identification and characterization of a set of conserved and new regulators of cytoskeletal organization, cell morphology and migration. BMC Biol, 2011. 9: p. 54. 
51. Mader, I., et al., Identification of a novel proapoptotic function of resveratrol in fat cells: SIRT1-independent sensitization to TRAIL-induced apoptosis. Faseb J, 2010. 24(6): p. 1997-2009.

52. Furukawa, A., et al., $\mathrm{H} 2 \mathrm{O} 2$ accelerates cellular senescence by accumulation of acetylated $\mathrm{p} 53$ via decrease in the function of SIRT1 by NAD+ depletion. Cell Physiol Biochem, 2007. 20(1-4): p. 45-54.

53. Mariman, E.C., An adipobiological model for weight regain after weight Loss. Adipobiology, 2011. 3.

54. Mariman, E.C. and P. Wang, Adipocyte extracellular matrix composition, dynamics and role in obesity. Cell Mol Life Sci, 2010. 67(8): p. 1277-92.

55. Lieber, J.G. and R.M. Evans, Disruption of the vimentin intermediate filament system during adipose conversion of 3T3-L1 cells inhibits lipid droplet accumulation. J Cell Sci, 1996. 109 ( Pt 13): p. 3047-58.

56. Hsu, D.K., R.Y. Yang, and F.T. Liu, Galectins in apoptosis. Methods Enzymol, 2006. 417: p. 256-73.

57. Ungvari, Z., et al., Resveratrol attenuates mitochondrial oxidative stress in coronary arterial endothelial cells. Am J Physiol Heart Circ Physiol, 2009. 297(5): p. H1876-81.

58. Low, F.M., et al., Peroxiredoxin 2 functions as a noncatalytic scavenger of low-level hydrogen peroxide in the erythrocyte. Blood, 2007. 109(6): p. 2611-7.

59. Kim, S.Y., T.J. Kim, and K.Y. Lee, A novel function of peroxiredoxin 1 (Prx-1) in apoptosis signal-regulating kinase 1 (ASK1)-mediated signaling pathway. FEBS Lett, 2008. 582(13): p. 1913-8.

60. Morinaka, A., et al., Oligomeric peroxiredoxin-I is an essential intermediate for p53 to activate MST1 kinase and apoptosis. Oncogene, 2011. 30(40): p. 4208-18.

61. Harman, D., Aging: a theory based on free radical and radiation chemistry. J Gerontol, 1956. 11(3): p. 298-300.

62. Huang, S., et al., Insulin stimulates membrane fusion and GLUT4 accumulation in clathrin coats on adipocyte plasma membranes. Mol Cell Biol, 2007. 27(9): p. 345669.

63. Kampinga, H.H. and E.A. Craig, The HSP70 chaperone machinery: J proteins as drivers of functional specificity. Nat Rev Mol Cell Biol, 2010. 11(8): p. 579-92.

64. Baur, J.A. and D.A. Sinclair, Therapeutic potential of resveratrol: the in vivo evidence. Nat Rev Drug Discov, 2006. 5(6): p. 493-506.

65. Famulla, S., et al., Pigment epithelium-derived factor (PEDF) is one of the most abundant proteins secreted by human adipocytes and induces insulin resistance and inflammatory signaling in muscle and fat cells. Int J Obes (Lond), 2011. 35(6): p. 76272. 
66. Mandal, P., et al., The anti-inflammatory effects of adiponectin are mediated via a heme oxygenase-1-dependent pathway in rat Kupffer cells. Hepatology, 2010. 51(4): p. 1420-9.

67. Baitsch, D., et al., Apolipoprotein $\mathrm{E}$ induces antiinflammatory phenotype in macrophages. Arterioscler Thromb Vasc Biol, 2011. 31(5): p. 1160-8.

68. Odrowaz-Sypniewska, G., Markers of pro-inflammatory and pro-thrombotic state in the diagnosis of metabolic syndrome. Adv Med Sci, 2007. 52: p. 246-50.

69. Wang, J.J., et al., Anti-inflammatory effects of pigment epithelium-derived factor in diabetic nephropathy. Am J Physiol Renal Physiol, 2008. 294(5): p. F1166-73.

70. Nieuwdorp, M., et al., Hypercoagulability in the metabolic syndrome. Curr Opin Pharmacol, 2005. 5(2): p. 155-9.

71. Tarnus, E., et al., Apolipoprotein E limits oxidative stress-induced cell dysfunctions in human adipocytes. FEBS Lett, 2009. 583(12): p. 2042-8.

72. Espiritu, D.J. and T. Mazzone, Oxidative stress regulates adipocyte apolipoprotein e and suppresses its expression in obesity. Diabetes, 2008. 57(11): p. 2992-8.

73. Chen, B., et al., Hypoxia dysregulates the production of adiponectin and plasminogen activator inhibitor-1 independent of reactive oxygen species in adipocytes. Biochem Biophys Res Commun, 2006. 341(2): p. 549-56.

74. Yu, B., C. Cook, and N. Santanam, The aporphine alkaloid boldine induces adiponectin expression and regulation in 3T3-L1 cells. J Med Food, 2009. 12(5): p. 1074-83.

75. Kadowaki, T., et al., Adiponectin and adiponectin receptors in insulin resistance, diabetes, and the metabolic syndrome. J Clin Invest, 2006. 116(7): p. 1784-92.

76. Sharma, A.M. and V.T. Chetty, Obesity, hypertension and insulin resistance. Acta Diabetol, 2005. 42 Suppl 1: p. S3-8.

77. Wang, P., et al., Plasma pigment epithelium-derived factor is positively associated with obesity in Caucasian subjects, in particular with the visceral fat depot. Eur $\mathrm{J}$ Endocrinol, 2008. 159(6): p. 713-8.

78. Borg, M.L., et al., Pigment epithelium-derived factor regulates lipid metabolism via adipose triglyceride lipase. Diabetes, 2011. 60(5): p. 1458-66.

79. Skurk, T. and H. Hauner, Obesity and impaired fibrinolysis: role of adipose production of plasminogen activator inhibitor-1. Int J Obes Relat Metab Disord, 2004. 28(11): p. 1357-64. 


\section{Chapter 7}

Calorie restriction-induced changes in the secretome of human adipocytes, comparision with resveratrol-induced secretome effects

Anja Rosenow, Jean-Paul Noben, Martin Wabitsch, Edwin C.M. Mariman, Johan Renes submitted 


\title{
CHAPTER 7
}

\begin{abstract}
Obesity is characterized by excess energy storage which results in dysfunctional white adipose tissue (WAT) and in turn leads to metabolic diseases. Lifestyle changes especially calorie restriction $(\mathrm{CR})$ reduces the risk for age and obesity-associated complications. The impact of CR on obesity has mainly been examined with human intervention studies, which indicated alterations in circulating adipokines. However, a detailed understanding of CRinduced adipocyte secretome changes remains elusive. Therefore, we investigated the effect of $\mathrm{CR}$ on the secretion profile of mature human adipocytes by using proteomics technology. We demonstrated CR-mediated adipocyte triglyceride reduction, which resulted in a positive effect on adipokine secretion indicating an improved inflammatory phenotype and alleviation of obesity-associated metabolic dysfunction including insulin resistance and glucose intolerance. Furthermore, 6 novel adipocyte-secreted proteins were identified which were regulated by CR. Since resveratrol (RSV) mimics CR we compared results from this study with data from our previous RSV study on the adipocyte secretome. We observed that both treatment strategies lead to a less inflammatory phenotype and an altered adipokine profile indicating improvement of metabolic complications even though the CR and RSV adipocyte secretomes differed from each other. Mass spectrometry data have been deposited to the ProteomeXchange with identifier PXD000301.
\end{abstract}




\section{Introduction}

The two major functional roles of white adipose tissue (WAT) are energy storage as triglycerides (TG) and endocrine/paracrine regulation of the energy metabolism. The endocrine and paracrine functions of the WAT are mediated by expression and release of secretory factors, known as adipokines, which include extracellular matrix proteins, hormones, neurotrophins, cytokines as well as proteins involved in angiogenesis and lipid and glucose metabolism [1-3]. Excess energy storage results in elevated fat mass, which is characterized by morphological, histological and functional changes of the WAT, including the alteration of secreted adipokines. These changes lead to dysfunction of the WAT, which is associated with obesity-related disorders such as type 2 diabetes mellitus, coronary heart disease and cancers $[2,4,5]$.

Bariatric surgery, drugs, exercise and/or calorie restriction (CR) are common methods to treat obesity and its complications. Especially CR has been shown to lower the risk for ageand obesity-associated diseases, which results in improved life quality and longer lifespan [6, 7]. In yeast, these effects are mediated by the silent information regulator 2 (SIR2) [8]. Beneficial effects of $C R$ in mammals have been attributed to SIRT1, the mammalian homologue of SIR2 [9]. Within mouse 3T3-L1 adipocytes the lipolysis of TG and the release of free fatty acid (FFA) are regulated by SIRT1 [9].

The effects of CR on obesity and its associated complications have been mainly investigated with human intervention studies. Varady et al. [10] demonstrated an improvement of the circulating adipokine profile together with a decreased adipocyte size already by a weight loss of $5 \%$ in severely obese women. Such improvements may be attributed to the decreased adipocyte size since adipokine expression and adipocyte size are correlated [11]. Yet, the molecular mechanisms of CR-mediated beneficial effects on adipokine expression and release are still poorly understood [12].

Simpson-Golabi-Behmel-Syndrome (SGBS) fat cells provide an ideal model to investigate changes in the secretome of human adipocytes [3]. As such, we analysed the effect of CR on mature adipocytes to investigate the relation between reduction of the TG content and changes in the adipokine secretion pattern. Therefore, mature SGBS adipocytes were calorie restricted by using serum free low caloric medium for a period of 4 days. The differences of the secretome of fully differentiated calorie restricted or non-calorie restricted SGBS adipocytes were analyzed by two-dimensional gel electrophoresis (2-DE). The identification of the secreted proteins was performed by liquid chromatography-electrospray ionization tandem mass spectrometry (LC-ESI-MS/MS).

Previously, we showed that resveratrol (RSV) has a positive effect on the SGBS secretome [13]. Recent studies have demonstrated that RSV can mimic positive effects of CR in obese persons [13-15]. These included improvement of insulin resistance, decreased levels of 


\section{CHAPTER 7}

blood glucose, TG and cytokines as well as increased intramyocellular lipid levels, decreased intrahepatic lipid content and decreased systolic blood pressure [14, 15]. Such RSV-induced beneficial health effects are also thought to be mediated by the activation of SIRT1 [16]. This suggests that CR and RSV may exert their beneficial effects on adipocytes by a common mechanism. It prompted us to compare the data from this CR study with data from our previous RSV study with respect to the effect of both treatments on the human adipokine profile.

\section{Methods}

\section{Materials}

Modified phenol red free DMEM/F12 medium without glucose, $0.5 \%$ trypsin-EDTA and $10,000 \mathrm{u} / \mathrm{ml}$ Penicillin/Streptomycin were obtained from Life Technologies (Bleiswijk, The Netherlands). Fetal bovine serum (FBS) was purchased from Bodinco (Alkmaar, The Netherlands). Additional cell culture supplements, protease inhibitor cocktail, phenylmethanesulfonylfluoride (PMSF), DL-Dithiothreitol (DTT), 3-[(3-cholamidopropyl) dimethyl-amonio]-1-propanesulfonate (CHAPS), a-cyano-4-hydroxyl-cinnamic acid (CHCA), trifluoroacetic acid (TFA) and acetonitrile (ACN) were purchased from Sigma-Aldrich (Zwijndrecht, The Netherlands). Immobilized pH gradient (IPG) buffer (pH 3-11, nonlinear), Dry-Strip cover fluid and immobiline Dry-Strip ( $\mathrm{pH} 3-11$, nonlinear, $24 \mathrm{~cm}$ ) were obtained from GE Healthcare (Diegem, Belgium).

\section{Cell culture}

Human Simpson-Golabi-Behmel syndrome (SGBS) cells were obtained from Prof. Dr. M. Wabitsch (University of Ulm, Germany) [17] and cultured as described [13]. 90\% confluent preadipocytes $\left(2.2 \pm 0.36 \times 10^{6}\right.$, mean $\left.\pm S D, n=3\right)$ were differentiated into mature adipocytes during 14 days as described [13]. On average $78 \%$ of the preadipocytes differentiated into mature adipocytes. To determine cell numbers, adipocytes were counted with a raster ocular. Preadipocytes were trypsinized and counted with a haemocytometer.

\section{CR experiments}

At first dose-response experiments were performed to define the optimal glucose concentration to study the effect of $\mathrm{CR}$ on the adipocyte secretome. Therefore, mature adipocytes (14 days differentiated) were cultured with $66 \mathrm{nM}$ insulin and with different glucose concentrations $(0,0.2,0.55$ and $1 \mathrm{mM}$ glucose $)$ for a period of $96 \mathrm{~h}$. The control cells reflected the time point of the beginning of the $C R$ intervention. As such, the control adipocytes were cultured for only 14 days after the initiation of differentiation. On day 12 of 
the differentiation the non-calorie restricted adipocytes were washed twice and incubated with phenol red free DMEM/F12 medium containing $17.5 \mathrm{mM}$ glucose (normal glucose condition) and $66 \mathrm{nM}$ insulin for $48 \mathrm{~h}$. These cells are indicated as non-CR adipocytes. In addition, mature adipocytes (14 days differentiation) cultured with $66 \mathrm{nM}$ insulin and the normal medium glucose concentrations of $17.5 \mathrm{mM}$ glucose were used to demonstrate the maximum TG accumulation within the 96h incubation period. The effect of CR on the adipocytes was measured by intracellular TG contents and glycerol release.

In the subsequent 2-DE experiments, non-CR adipocytes were compared with $0.55 \mathrm{mM} \mathrm{CR}$ adipocytes the here assessed adequate glucoses concentration to achieve ideal CR conditions. As such, 14 days differentiated adipocytes were washed twice and cultured with $0.55 \mathrm{mM}$ glucose and $66 \mathrm{nM}$ insulin in phenol red free DMEM/F12 medium for 4 days.

\section{Measurement of TG content by ORO staining}

Adipocytes were fixed with $3.7 \%$ formaldehyde for $10 \mathrm{~min}$ at room temperature. Fixed cells were incubated with a filtered ORO solution (1\% in $60 \%$ isopropanol) for 30 min. Finally, cells were washed 6 times with $70 \%$ ethanol. TG accumulation was visualized with a Nikon TE 200 eclipse phase contrast microscope equipped with digital image acquisition. To determine intracellular TG accumulation, the ORO stain was extracted with dimethylsulfoxide (DMSO) and measured by spectrophotometry at $540 \mathrm{~nm}$. The amount of intracellular ORO content was corrected for the number of cells.

\section{Determination of glycerol release}

Glycerol released in the culture medium after 4 days of CR was measured by a fluorimetric assay using a Cobas-Fara Instrument (Roche Diagnostics, Almere, The Netherlands). The amount of glycerol release was corrected for the number of cells.

\section{Measurement of hormone sensitive lipase (HSL) and adipose triglyceride lipase (ATGL) by Western blotting}

To obtain cellular protein material cells were solubilised in lysis buffer consisting of $8 \mathrm{M}$ urea, $2 \% \mathrm{w} / \mathrm{v}$ CHAPS and $65 \mathrm{mM}$ DTT and a protease inhibitor cocktail. The protein concentrations were determined by using a Bradford-based protein assay kit (BioRad Laboratories, Veenendaal, The Netherlands). Cellular proteins $(10 \mu \mathrm{g})$ were separated by $10 \%$ SDS gel electrophoresis and then transferred to a nitrocellulose membrane. The membrane was blocked with $5 \%$ milk and probed with the polyclonal rabbit antibody against human HSL (kind gift from Prof. Dr. C Holm, Lund University, Sweden) or with the polyclonal rabbit antibody against ATGL (Cell Signalling Technology, Leiden, The Netherlands). Afterwards, the membrane was subjected to polyclonal swine anti rabbit IgG (DAKO, Glostrup, Denmark) 


\section{CHAPTER 7}

as secondary antibodies. Bound antibodies were visualized with the enhanced chemiluminescence reagent SuperSignal west Dura extended duration reagent (Pierce/Thermo Scientific, Etten-Leur, The Netherlands).

\section{Sample preparation for 2-DE experiments}

Culture media were collected, supplemented with protease inhibitor cocktail, transferred to dialysis tubes (2-kDa molecular-weight cut-off, Carl Roth $\mathrm{GmbH}$, Karlsruhe, Germany) and dialyzed against $20 \mathrm{mM}$ ammonium bicarbonate at $4^{\circ} \mathrm{C}$ for $48 \mathrm{~h}$ with $10-12 \mathrm{x}$ buffer changes. The dialyzed medium samples were freeze-dried, dissolved in fresh rehydration buffer (8 M urea, $2 \% \mathrm{w} / \mathrm{v}$ CHAPS and $65 \mathrm{mM} \mathrm{DTT}$ ) and centrifuged at $20000 \mathrm{xg}$ at $10^{\circ} \mathrm{C}$ for $30 \mathrm{~min}$. The protein concentrations were determined with a Bradford-based protein assay kit (Bio-Rad Laboratories). All samples were stored at $-80^{\circ} \mathrm{C}$.

\section{2-DE}

Protein samples derived from $2.75 \times 10^{6}$ adipocytes were used for 2-DE analysis according to Bouwman et al. [18] but with different IPG strips ( $\mathrm{pH} \mathrm{3-11,} \mathrm{nonlinear,} 24 \mathrm{~cm}$ ). $100 \mathrm{~g}$ of total protein in a volume of 450 I containing $0.5 \%(\mathrm{v} / \mathrm{v})$ IPG was loaded onto the IPG strips. For protein profiling, 4 independent replicates were made for $\mathrm{CR}(0.55 \mathrm{mM}$ glucose concentration for a period of $96 \mathrm{~h})$ and non-CR (17.5 mM glucose concentration) adipocytes. The gels were stained with Flamingo fluorescent gel stain according to the manufacturer's protocol (Bio-Rad Laboratories). To visualize the proteins, gels were scanned by the Molecular Imager FX (BioRad Laboratories).

\section{Image analysis}

Stained gels were processed by PDQuest 8.0 software (Bio-Rad Laboratories). Data were normalized with respect to the total density of gel image. CR and non-CR groups were formed from samples of 4 independent experiments with the same treatment. Protein spots were regarded as significantly differentially expressed if the average spot intensity between the $\mathrm{CR}$ and non-CR groups differed more than 1.5-fold with $p<0.05$ (Student's T-test). The criteria for indicating a trend was a spot intensity difference of more than 1.5-fold with $0.05<p<0.1$. For subsequent protein identification, gels with differentially expressed spots were re-stained with SYPRO Ruby Protein Stain according to the manufacturer's protocol (Bio-Rad Laboratories).

\section{MS/MS and protein identification}

Excised spots were in-gel digested as described by Bouwman et al. [18]. $10 \mu \mathrm{l}$ of the digested spots were analyzed by LC-ESI MS/MS on a LCQ Classic (Thermo Scientific, San 
Jose, USA), as described by Dumont et al. [19]. The trapped sample was separated on the analytical column (Biosphere C18, $5 \mu \mathrm{m}$ particle diameter, $200 \mathrm{~mm} \mathrm{~L} \times 0.05 \mathrm{~mm}$ ID; Nanoseparations, Nieuwkoop, The Netherlands) using a linear gradient from 5 to $60 \% \mathrm{v} / \mathrm{v}$ ACN in water containing $100 \mathrm{mM}$ acetic acid in $55 \mathrm{~min}(100 \mathrm{nl} / \mathrm{min})$. The eluate of the analytical column was nanosprayed from a teflon-connected, gold-coated fused silica emitter (5 $\mu \mathrm{m}$ ID; NanoSeparations, Nieuwkoop, The Netherlands).

\section{Database search}

With respect to LC-ESI-MS/MS, LCQ Xcalibur v2.0 SR2 raw files and spectra were selected from Proteome Discover1.2 software (Thermo Scientific) with the following settings: minimal peak count 50 , total intensity threshold 4000 and $S / N \geq 6$. Peak lists were searched with Sequest v1.2.0.208 and Mascot v2.3.0.1 against EMBL-EBI International Protein Index database for human proteins (version $3.78,86702$ entries) by using the following settings: fragment tolerance, 1.00 Da (monoisotopic); parent tolerance, 3.0 Da (monoisotopic); fixed modifications, carbamidomethylation of cystein; variable modifications, oxidation of methionin; max missed cleavages, 2. Search engine results were combined and validated by Scaffold v3.00.07 (Proteome Software, Portland, OR, USA) with minimum peptide and protein probability set to $\geq 80 \%$, followed by visual inspection of spectral (annotation) quality and manual curation, eliminating high-background spectra and single-engine identifications, evaluating one-hit wonders and filtering out keratins. The mass spectrometry proteomics data have been deposited to the ProteomeXchange Consortium (http://proteomecentral.proteomexchange.org<http://proteomecentral.proteomexchange.org> ) via the PRIDE partner repository [20] with the dataset identifier "PXD000301 and DOI 10.6019/PXD000301." Mascot DAT files were processed by PRIDE Converter software [21] and submitted via ProteomeXchange (http://www.proteomexchange.org) to the PRIDE database [22], project accession number: 29652-29779. MS data can be visualized using PRIDE Inspector [23] (http:tinyurl.com/csffalc) and Scaffold .sf3 free viewer (https://proteomecommons.org/tool.jsp?i=1009).

\section{Analysis of secreted protein candidates}

For verification of secreted protein candidates an amino acid sequence analysis was performed with SignalP 3.0 and SecretomeP 2.0 (CBS, Technical University Copenhagen, Denmark, URL: www.cbs.tdu.dk). SignalP 3.0 was used to verify the presence and location of signal peptide cleavage sites in amino acid sequences. SecretomeP 2.0 was applied to categorize proteins as non-classical secreted in case they were devoid of a signal peptide but obtained an NN-score above the threshold of 0.5 . 


\section{CHAPTER 7}

\section{Statistical analysis}

Statistical analyses of protein identifications were performed with the Student's T-test. Glycerol release and TG accumulation was analyzed by ANOVA. Significant differences between groups were indicated with $p<0.05$.

\section{Results}

\section{CR-induced lipolysis in SGBS adipocytes}

CR-induced lipolysis in SGBS cells was determined by measurement of intracellular TG content and glycerol release under low glucose conditions $(0,0.2,0.55$ and $1.0 \mathrm{mM})$. Compared to control cells a significant reduction of intracellular TG was observed with 0.2 $\mathrm{mM}$ (reduction of $25 \pm 0.03 \%, \mathrm{n}=4$ ) and $0.55 \mathrm{mM}$ (reduction of $17 \pm 0.06 \%, \mathrm{n}=4$ ) glucose (Figure $1 \mathrm{~A}$ and $1 \mathrm{~B}$ ). With respect to glycerol, a significant decrease of glycerol release was observed with $0 \mathrm{mM}$ (reduction of $88 \pm 0.08 \%, \mathrm{n}=4$ ), $0.2 \mathrm{mM}$ (reduction of $90 \pm 0.06 \%, \mathrm{n}=4$ ) and $0.55 \mathrm{mM}$ (reduction of $81 \pm 0.14 \%, \mathrm{n}=4$ ) glucose compared to the control (Figure $1 \mathrm{C}$ ). To investigate the maximum CR-induced secretome changes of mature SGBS adipocytes, we chose a $0.55 \mathrm{mM}$ glucose concentration. In addition, Western Blotting results from 4 independent experiments showed that after 4 days of $0.55 \mathrm{mM}$ treatment ATGL expression was significant down-regulated while HSL expression was up to $60 \%$ up-regulated compared to the control condition (Figure 1D). 
A

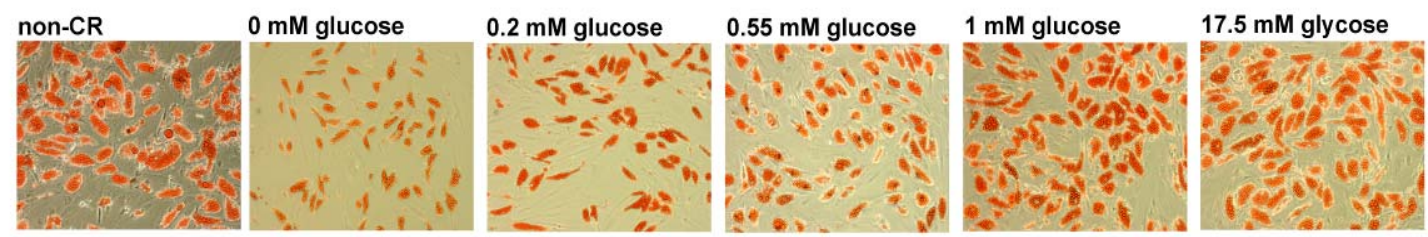

B

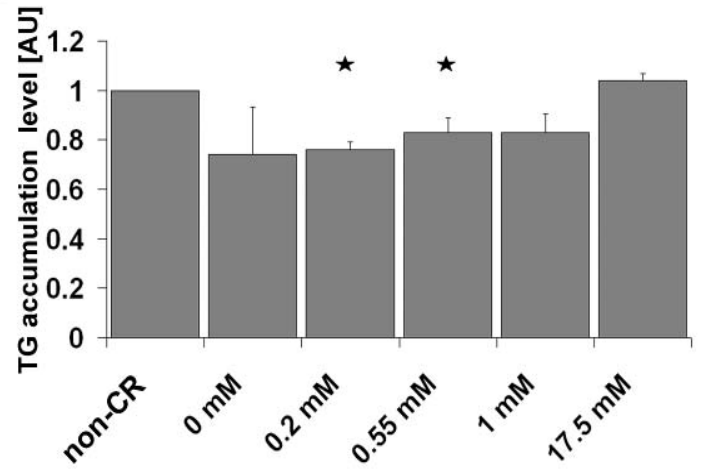

C

$\mathrm{D}$
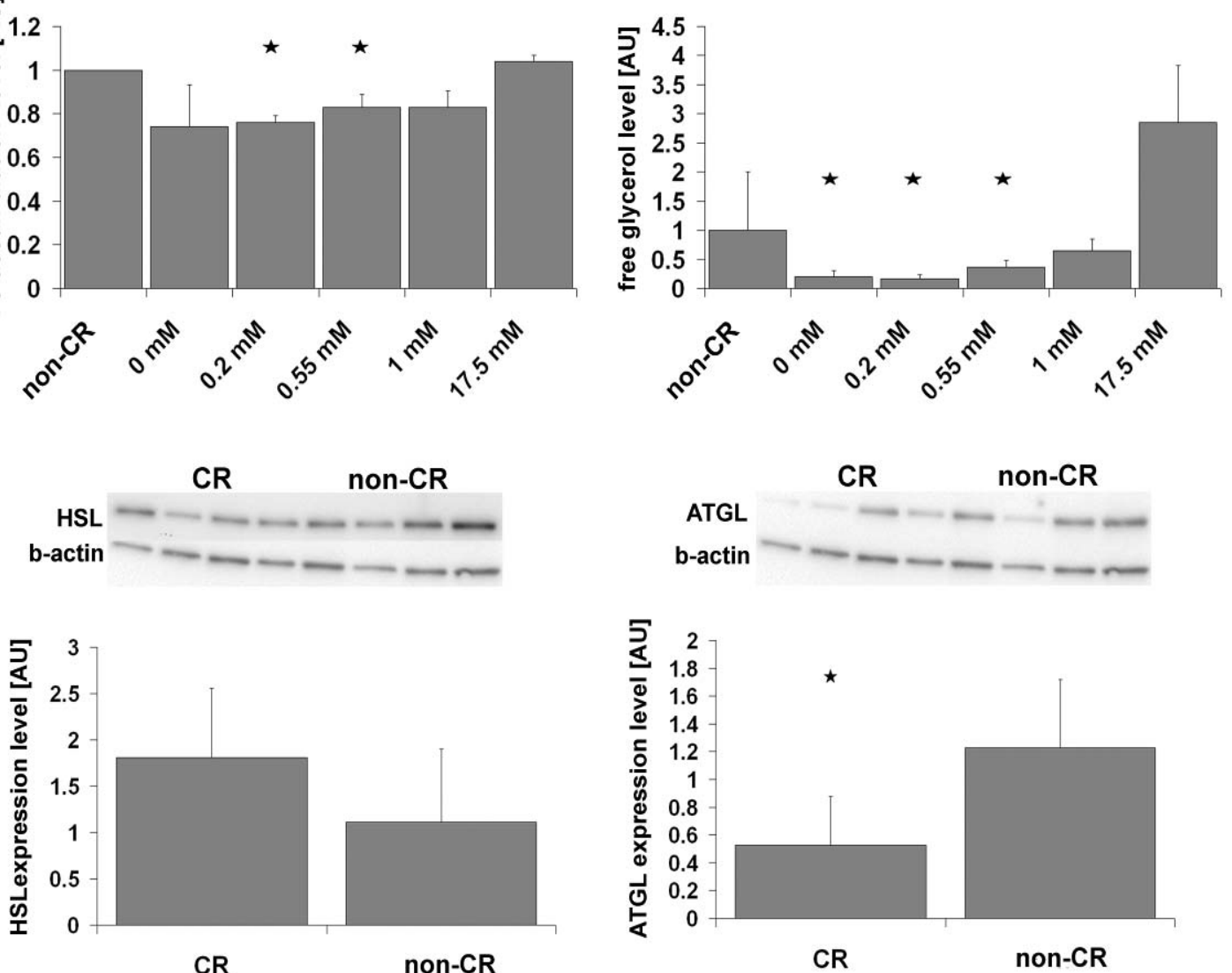

Figure 1: Glycerol release and TG accumulation studies of calorie-restricted adipocytes. A: Images with a Nikon TE 200 eclipse phase contrast microscope of 14 days differentiated adipocytes which were not calorie restricted (non-CR) or calorie restricted for 4 days with different concentrations of medium glucose. From left to right: non-CR, 0, 0.2, 0.55, 1.0 and $17.5 \mathrm{mM}$ medium glucose. B: Intracellular TG accumulation determined by spectrophotometry after similar conditions as in A. C: Glycerol release in the culture medium determined after similar conditions as in A. D: HSL and ATGL protein levels in 4 days calorie restricted ( $0.55 \mathrm{mM}$ glucose) adipocytes, compared to non-CR adipocytes. Western blotting analysis was performed with $10 \mu \mathrm{g}$ of total protein lysate. Normalization was done by using $\beta$-actin protein signal. Reported values are means \pm SD of 3 independent biological replications and * indicates differences with $p<0.05$.

\section{CR-induced secretome changes of SGBS adipocytes}

CR-induced secretome changes were identified by comparing $100 \mu \mathrm{g}$ of secreted protein samples derived from $\mathrm{CR}$ adipocytes and non-CR adipocytes. Protein samples were separated by 2-DE and analyzed by PDQuest. Representative gels of the non-CR and the $\mathrm{CR}$ condition together with the master gel are shown in Figure 2. 
A
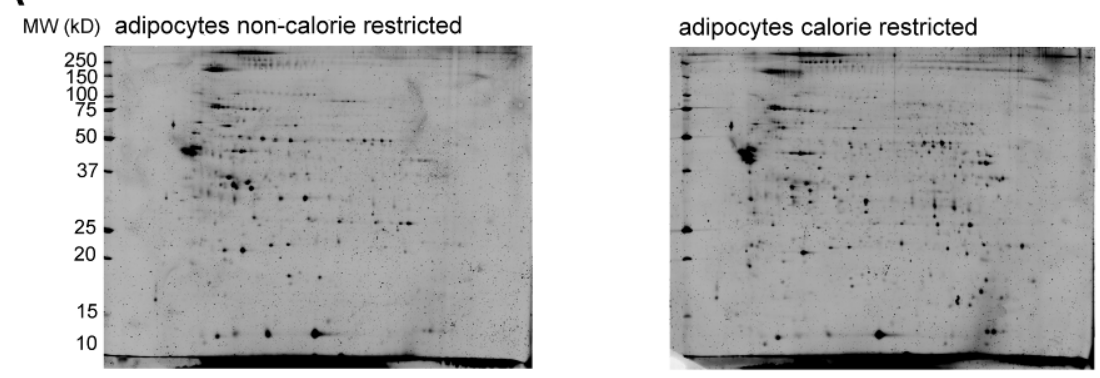

B

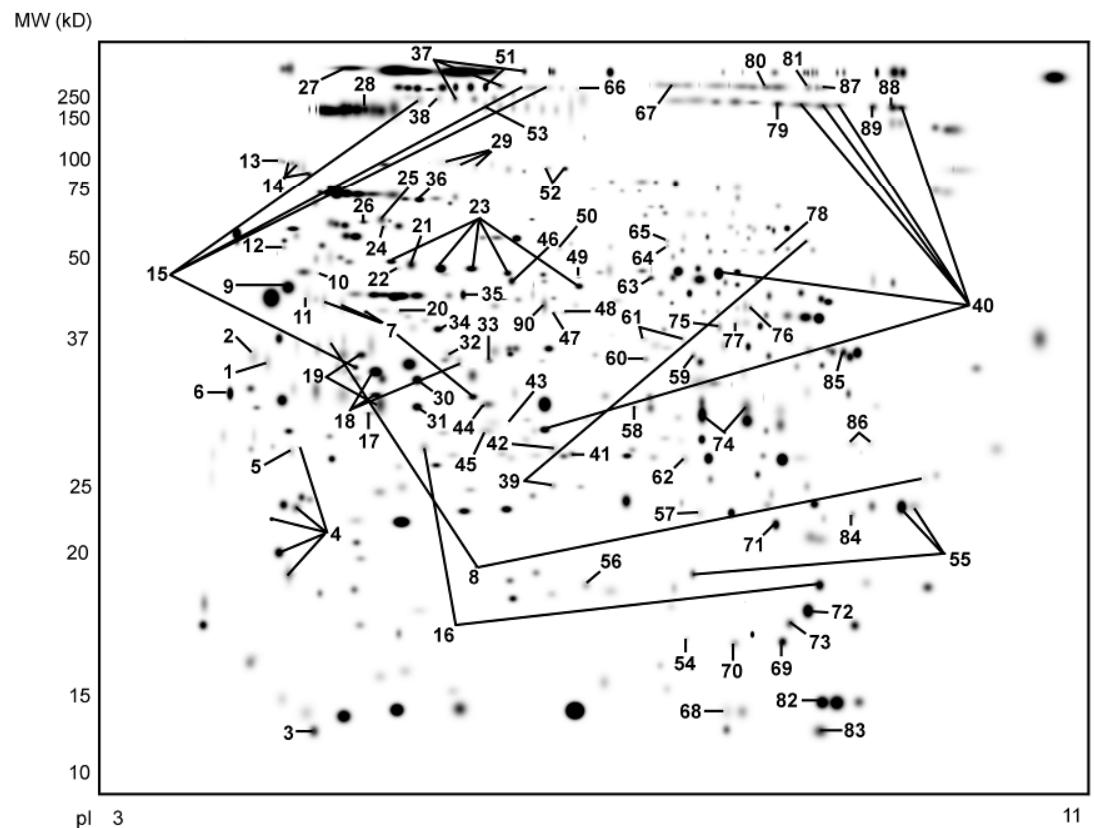

Figure 2: 2-DE gels of adipocyte secreted proteins. A: Representative gels of mediumderived secreted proteins of non-CR adipocytes (left) and 4 days calorie restricted $(0.55$ $\mathrm{mM}$ glucose) adipocytes (right). B: Master gel with identified spots. Spot numbers refer to ID number of Table 1.

From a total of 511 matched spots, 141 spots were either significantly ( $p<0.05$ : 92 spots) or as a trend ( $p<0.1: 49$ spots) differentially expressed. These spots were excised from the gels and analyzed by LC-ESI-MS/MS. 127 of the 141 spots were identified. Subsequent analysis revealed 89 unique proteins (Figure 2 and Supplement 1). SignalP 3.0 and SecretomeP 2.0 analysis of the 89 proteins revealed 34 proteins as classical secreted proteins, 22 proteins as non-classical secreted and 33 proteins as intracellular proteins (Supplement 1). Identical proteins could be identified in different spots containing either one or several different proteins. As such, proteins with similar accession numbers can be related to different IDnumbers dependent on the number of different proteins within one spot. Therefore, protein identifications derived from one protein per spot were listed in Table 1. From those proteins a clear CR-mediated regulation pattern could be obtained. The regulation pattern of proteins identified from spots containing more than one protein remains elusive. These are listed in 
Table 2. Next to secretory proteins intracellular proteins were also detected in non-single protein spots, these are listed in Supplement 1.

The category of classical secreted proteins was further sub-categorized into extracellular matrix (ECM) proteins (9), processing (4), regulation/signalling proteins (18) and immune regulation proteins (3) (Tables 1 and 2, Supplement 1 ).

\begin{tabular}{|c|c|c|c|c|c|}
\hline $\begin{array}{l}\text { Accession } \\
\text { number }\end{array}$ & Protein name & ID & $\begin{array}{c}\mathrm{MW} \\
{[\mathrm{kDa}]}\end{array}$ & $\begin{array}{c}\text { fold } \\
\text { change }\end{array}$ & p-value \\
\hline \multicolumn{6}{|c|}{$\begin{array}{l}\text { classical secreted } \\
\text { extracellular matrix }\end{array}$} \\
\hline P02452 & Collagen alpha-1(I) chain & $\begin{array}{l}37 \\
37 \\
37\end{array}$ & $\begin{array}{l}219.3 \\
221.0 \\
251.4\end{array}$ & $\begin{array}{r}-2.4 \mathrm{E}+00 \\
-3.9 \mathrm{E}+00 \\
7.9 \mathrm{E}+00\end{array}$ & $\begin{array}{l}9.32 \mathrm{E}-02 \\
4.27 \mathrm{E}-02 \\
2.87 \mathrm{E}-02\end{array}$ \\
\hline P02461 & Collagen alpha-1(III) chain & 30 & 33.4 & $-2.9 E+00$ & 1.83E-02 \\
\hline P20908 & Collagen alpha- $1(\mathrm{~V})$ chain & 2 & 36.0 & $-4.4 \mathrm{E}+00$ & 7.60E-02 \\
\hline P12109 & Collagen alpha-1(VI) chain & 28 & 196.7 & $-1.3 E+00$ & 2.25E-02 \\
\hline \multirow[t]{6}{*}{ P08123 } & Collagen alpha-2(I) chain & 40 & 28.7 & $6.1 E+04$ & 1.30E-06 \\
\hline & & 40 & 48.4 & $2.1 \mathrm{E}+00$ & $9.20 \mathrm{E}-02$ \\
\hline & & 40 & 206.3 & $7.6 \mathrm{E}+00$ & 1.95E-02 \\
\hline & & 40 & 203.2 & $5.7 E+00$ & 5.05E-02 \\
\hline & & 40 & 205.2 & $2.5 E+01$ & 4.55E-02 \\
\hline & & 40 & 199.7 & $1.3 E+01$ & 4.82E-02 \\
\hline P08572 & Collagen alpha-2(IV) chain & 87 & 247.8 & $-5.7 E+00$ & 2.49E-03 \\
\hline P12110 & Collagen alpha-2(VI) chain & 53 & 201.8 & $-4.8 E+00$ & 2.23E-02 \\
\hline \multirow[t]{2}{*}{ P02751 } & Fibronectin & 27 & 308.5 & $-8.7 E+00$ & 2.67E-03 \\
\hline & pro & & & & \\
\hline P07711 & Cathepsin L1 & 34 & 39.7 & $5.3 E+04$ & $6.62 \mathrm{E}-02$ \\
\hline \multirow[t]{2}{*}{ P01033 } & Metalloproteinase inhibitor 1 & 74 & 30.3 & $2.2 E+04$ & 8.49E-03 \\
\hline & & 74 & 29.5 & $1.9 \mathrm{E}+00$ & 5.05E-02 \\
\hline \multirow[b]{2}{*}{ P02649 } & regulation/signaling & & & & \\
\hline & Apolipoprotein E & $\begin{array}{l}8 \\
8\end{array}$ & $\begin{array}{l}24.6 \\
37.8\end{array}$ & $\begin{array}{r}4.9 \mathrm{E}+03 \\
-4.1 \mathrm{E}+00\end{array}$ & $\begin{array}{l}7.00 \mathrm{E}-07 \\
2.62 \mathrm{E}-02\end{array}$ \\
\hline D6QS48 & Calumenin (isoform 4) & 6 & 32.1 & $-3.8 E+00$ & 2.10E-02 \\
\hline P14625 & Endoplasmin & 12 & 58.1 & $6.7 E+03$ & 1.56E-02 \\
\hline \multirow[t]{2}{*}{ P06396 } & Gelsolin & 52 & 116.7 & $-2.0 \mathrm{E}+00$ & $7.80 \mathrm{E}-02$ \\
\hline & & 52 & 117.1 & $-6.4 \mathrm{E}+00$ & $9.41 \mathrm{E}-02$ \\
\hline P00738 & Haptoglobin & 38 & 16.7 & $-8.8 \mathrm{E}+00$ & 6.96E-02 \\
\hline \multirow[t]{5}{*}{ P36955 } & Pigment epithelium-derived factor & 23 & 51.3 & $-9.8 \mathrm{E}+00$ & 1.62E-02 \\
\hline & & 23 & 49.3 & $-2.5 \mathrm{E}+00$ & 6.77E-02 \\
\hline & & 23 & 49.5 & $-4.5 \mathrm{E}+00$ & 7.94E-03 \\
\hline & & 23 & 48.5 & $-4.0 \mathrm{E}+00$ & 1.04E-02 \\
\hline & & 23 & 46.3 & $1.1 \mathrm{E}+01$ & 3.82E-02 \\
\hline \multirow[t]{2}{*}{ P41222 } & Prostaglandin-H2 D-isomerase & 16 & 27.1 & $-1.5 E+01$ & 2.15E-02 \\
\hline & & 16 & 16.4 & $1.4 \mathrm{E}+05$ & 1.91E-03 \\
\hline P10599 & Thioredoxin & 3 & 9.3 & $3.4 \mathrm{E}+04$ & 7.21E-04 \\
\hline \multirow[t]{4}{*}{ P25311 } & Zinc-alpha-2-glycoprotein & 7 & 43.2 & $-9.3 E+00$ & 3.02E-02 \\
\hline & & 7 & 43.9 & $-8.4 E+00$ & 3.92E-02 \\
\hline & & 7 & 42.2 & $-9.0 \mathrm{E}+04$ & 9.62E-03 \\
\hline & & 7 & 31.7 & $9.2 \mathrm{E}+04$ & 1.83E-02 \\
\hline \multirow{4}{*}{ B4DPQ0 } & immune regulation & & & & \\
\hline & $\begin{array}{l}\text { cDNA FLJ54471. highly similar to Complement C1r } \\
\text { subcomponent* }^{*}\end{array}$ & 29 & 123.0 & $-4.0 E+00$ & 3.97E-02 \\
\hline & & 29 & 121.7 & $-4.5 E+00$ & 4.59E-02 \\
\hline & & 29 & 123.4 & $-2.5 E+00$ & 3.33E-02 \\
\hline \multirow[t]{4}{*}{ P09871 } & Complement C1s subcomponent & 14 & 122.2 & $-3.4 \mathrm{E}+00$ & 4.91E-02 \\
\hline & & 14 & 119.6 & $3.2 \mathrm{E}+00$ & 7.69E-02 \\
\hline & & 14 & 111.3 & $1.5 \mathrm{E}+04$ & 7.17E-06 \\
\hline & Protein S100-A9 & 51 & 249.6 & $8.1 E+00$ & 2.43E-03 \\
\hline
\end{tabular}


Table 1: Single protein per spot identifications of secreted proteins from CR and non-CR SGBS adipocytes

\begin{tabular}{|c|c|c|c|c|c|}
\hline $\begin{array}{c}\text { Accession } \\
\text { number }\end{array}$ & Protein name & ID & $\begin{array}{c}\mathrm{MW} \\
{[\mathrm{kDa}]}\end{array}$ & $\begin{array}{c}\text { fold } \\
\text { change }\end{array}$ & p-value \\
\hline Q6NVY1 & $\begin{array}{l}\text { non-classical secreted } \\
\text { 3-hydroxyisobutyryl-CoA hydrolase. mitochondrial } \\
\text { (Isoform 1)* }\end{array}$ & 75 & 40.1 & $-5.8 \mathrm{E}+00$ & 4.59E-02 \\
\hline P06733 & Alpha-enolase (Isoform alpha-enolase) & 78 & 56.5 & $-4.5 E+00$ & 5.79E-02 \\
\hline P23528 & Cofilin-1 & 72 & 14.8 & $2.6 E+00$ & 2.50E-02 \\
\hline P09104 & Gamma-enolase & 10 & 48.5 & $6.4 \mathrm{E}+03$ & $7.36 \mathrm{E}-02$ \\
\hline P04792 & Heat shock protein beta- 1 & 41 & 26.6 & $1.3 \mathrm{E}+00$ & $7.28 \mathrm{E}-02$ \\
\hline Q6NSB4 & HP protein & 90 & 43.2 & $-5.3 E+04$ & $1.22 \mathrm{E}-02$ \\
\hline P30086 & Phosphatidylethanolamine-binding protein 1 & 71 & 20.7 & $2.0 \mathrm{E}+00$ & $7.61 \mathrm{E}-02$ \\
\hline P28074 & Proteasome subunit beta type-5 (isoform 1) & 84 & 21.5 & $2.1 \mathrm{E}+00$ & 4.21E-02 \\
\hline P04179 & Superoxide dismutase [Mn]. mitochondrial & 57 & 21.6 & $-2.3 E+00$ & 3.74E-02 \\
\hline \multirow{3}{*}{ Q01995 } & Transgelin & 55 & 22.1 & $3.0 \mathrm{E}+04$ & 2.32E-04 \\
\hline & & 55 & 17.1 & $4.6 \mathrm{E}+00$ & $5.44 \mathrm{E}-02$ \\
\hline & & 55 & 22.1 & $2.0 \mathrm{E}+00$ & 7.95E-02 \\
\hline P15374 & Ubiquitin carboxyl-terminal hydrolase isozyme L3* & 5 & 26.9 & $2.9 E+00$ & $5.71 \mathrm{E}-02$ \\
\hline \multirow[t]{5}{*}{ P08670 } & Vimentin & 4 & 17.1 & $8.1 \mathrm{E}+00$ & $6.22 \mathrm{E}-02$ \\
\hline & & 4 & 21.1 & $6.7 \mathrm{E}+01$ & 5.32E-02 \\
\hline & & 4 & 18.6 & $1.9 E+00$ & 3.27E-02 \\
\hline & & 4 & 22.1 & $2.5 \mathrm{E}+00$ & 2.04E-02 \\
\hline & & 4 & 27.2 & $4.9 E+03$ & 1.99E-02 \\
\hline
\end{tabular}

Table 1: Single protein per spot identifications of secreted proteins from CR and non-CR SGBS adipocytes by 2-

DE followed by LC-MS/MS. Proteins marked with * are identified as novel adipocyte secreted proteins. ID numbers refers to marked spots of Figure 2B. MW of the protein spots are defined by PDQuest 8.0 software. Lines marked bolt are significant CR reactive proteins.

Analysis of the regulation patterns of proteins with similar functions revealed that a large group of structure-related proteins changed considerably by CR. As such, most of the subunits and isoforms of type I, III, IV, V and VI collagens and fibronectin were found to be down-regulated. In contrast, all isoforms of collagen 2(I) were induced by CR. In addition, the structural proteins including cofilin 1, transgelin and vimentin were also induced by CR. Spots of processing proteins as well as endoplasmin were up-regulated by CR.

$\mathrm{CR}$ also changed the secretion of proteins related to regulation and signalling. As such, most of the identified subunits and/or isoforms of apolipoprotein $E$ (ApoE), haptoglobin, pigment epithelium-derived factor (PEDF) and zinc-alpha-2-glycoprotein (ZAG) were down-regulated by $\mathrm{CR}$ (Table 1). In addition, many adipocyte-specific proteins including adiponectin, angiopoietin-related protein 1, angiotensinogen, calumenin, follistatin-related protein 1 , plasminogen activator inhibitor 1 (PAl-1) were identified after the CR intervention (Table 2). However, these proteins were found in a mixed spot, which makes their CR-mediated regulation pattern less clear. 
Table 2: Identifications from non-single protein spots of secreted proteins of CR and non-CR SGBS adipocytes

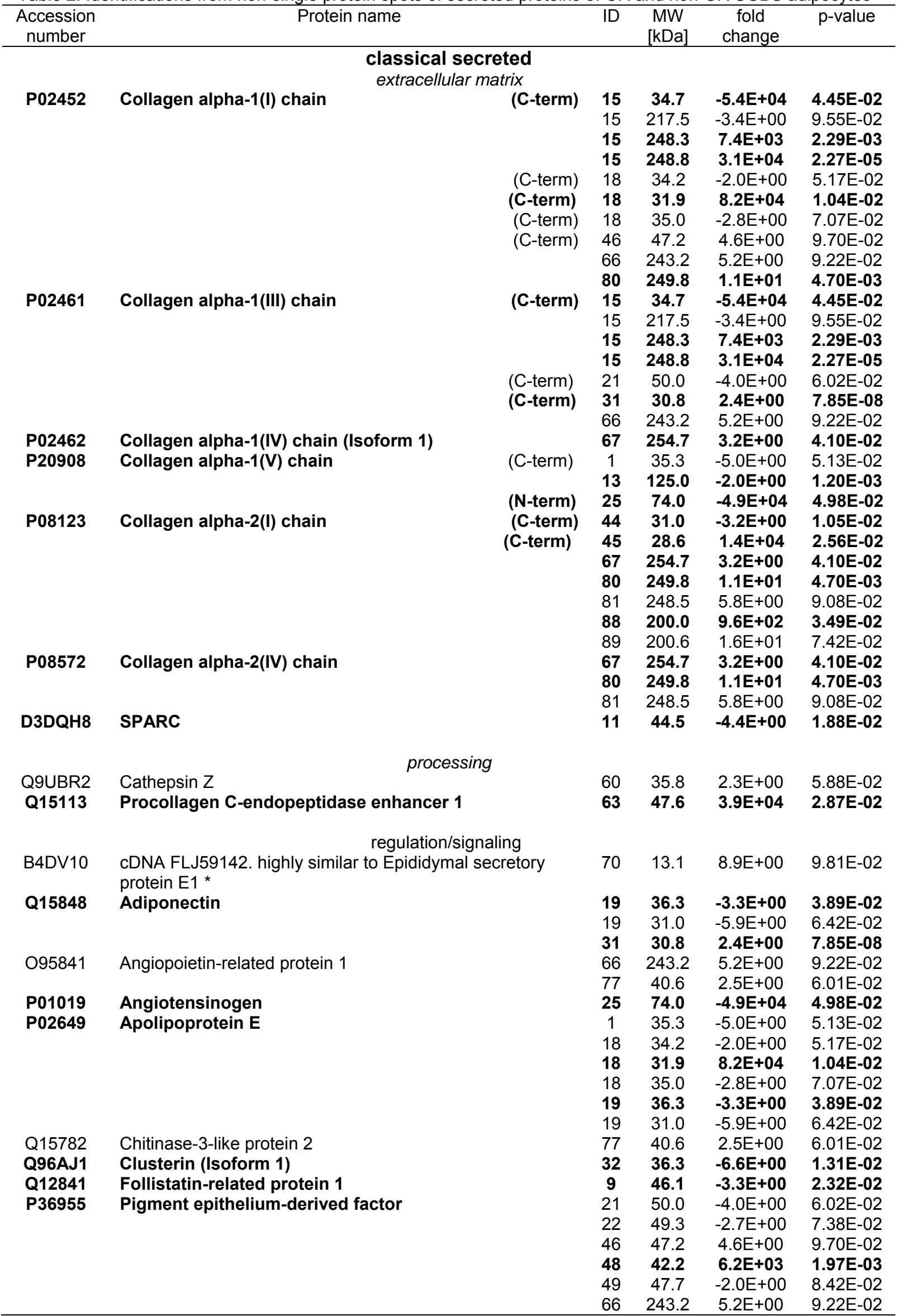


Table 2: Identifications from non-single protein spots of secreted proteins of CR and non-CR SGBS adipocytes

\begin{tabular}{|c|c|c|c|c|c|}
\hline $\begin{array}{l}\text { Accession } \\
\text { number }\end{array}$ & Protein name & ID & $\begin{array}{c}\mathrm{MW} \\
{[\mathrm{kDa}]}\end{array}$ & $\begin{array}{c}\text { fold } \\
\text { change }\end{array}$ & p-value \\
\hline \multicolumn{6}{|c|}{$\begin{array}{l}\text { classical secreted } \\
\text { regulation/signaling }\end{array}$} \\
\hline P05121 & Plasminogen activator inhibitor 1 & $\begin{array}{l}63 \\
76\end{array}$ & $\begin{array}{l}47.6 \\
42.8\end{array}$ & $\begin{array}{l}3.9 \mathrm{E}+04 \\
7.1 \mathrm{E}+03\end{array}$ & $\begin{array}{l}2.87 \mathrm{E}-02 \\
3.47 \mathrm{E}-02\end{array}$ \\
\hline P41222 & Prostaglandin-H2 D-isomerase & 42 & 27.0 & $1.4 \mathrm{E}+05$ & 8.17E-02 \\
\hline Q8NBS9 & Thioredoxin domain-containing protein 5 & 22 & 49.3 & $-2.7 E+00$ & 7.38E-02 \\
\hline P25311 & Zinc-alpha-2-glycoprotein & 11 & 44.5 & $-4.4 \mathrm{E}+00$ & $1.88 \mathrm{E}-02$ \\
\hline \multicolumn{6}{|c|}{ immune regulation } \\
\hline P09871 & Complement $\mathrm{C} 1 \mathrm{~s}$ subcomponent & 13 & 125.0 & $-2.0 E+00$ & $1.20 \mathrm{E}-03$ \\
\hline \multicolumn{6}{|c|}{ non-classical secreted } \\
\hline $\begin{array}{l}\text { P08865 } \\
\text { B4DWA6 }\end{array}$ & $\begin{array}{l}\text { 40S ribosomal protein SA } \\
\text { cDNA FLJ60094. highly similar to F-actin capping } \\
\text { protein subunit beta* }\end{array}$ & $\begin{array}{l}11 \\
44\end{array}$ & $\begin{array}{l}44.5 \\
31.0\end{array}$ & $\begin{array}{l}-4.4 \mathrm{E}+00 \\
-3.2 \mathrm{E}+00\end{array}$ & $\begin{array}{l}1.88 \mathrm{E}-02 \\
1.05 \mathrm{E}-02\end{array}$ \\
\hline P06733 & Alpha-enolase (Isoform alpha-enolase) & 49 & 47.7 & $-2.0 \mathrm{E}+00$ & 8.42E-02 \\
\hline P60981 & Destrin & 73 & 14.1 & $3.9 E+00$ & 5.04E-02 \\
\hline P15090 & Fatty acid binding protein. adipocyte & 68 & 10.1 & $6.5 E+04$ & 6.37E-02 \\
\hline P09211 & Glutathione S-transferase P & 39 & 24.0 & $8.3 E+00$ & 2.72E-02 \\
\hline P00390 & Glutathione reductase. mitochondrial & 39 & 61.3 & $4.3 E+03$ & 5.11E-02 \\
\hline Q6NSB4 & HP protein * & 35 & 44.9 & $-8.7 E+04$ & 1.75E-02 \\
\hline P40926 & Malate dehydrogenase. mitochondrial & 85 & 36.6 & $3.5 \mathrm{E}+01$ & $1.35 \mathrm{E}-02$ \\
\hline P30044 & Peroxiredoxin-5. mitochondrial & 70 & 13.1 & $8.9 E+00$ & $9.81 \mathrm{E}-02$ \\
\hline Q08257 & Quinone oxidoreductase & 85 & 36.6 & $3.5 E+01$ & 1.35E-02 \\
\hline P60174 & Triosephosphate isomerase (isoform 1) & 62 & 26.2 & $1.4 \mathrm{E}+04$ & 1.50E-02 \\
\hline \multirow[t]{4}{*}{ P08670 } & Vimentin & 9 & 46.1 & $-3.3 E+00$ & 2.32E-02 \\
\hline & & 11 & 44.5 & $-4.4 E+00$ & $1.88 \mathrm{E}-02$ \\
\hline & & 26 & 72.7 & $-6.6 E+01$ & 6.63E-02 \\
\hline & & 76 & 42.8 & $7.1 E+03$ & 3.47E-02 \\
\hline
\end{tabular}

Table 2: Multi-protein per spot identifications of secreted proteins from CR and non-CR SGBS adipocytes by 2-DE followed by LC-MS/MS. Proteins with the identical ID number are related to the spots with the same protein identifications and proteins marked with * are identified as novel adipocyte secreted proteins. ID numbers refers to marked spots of Figure 2B. MW of the protein spots are defined by PDQuest 8.0 software. Lines marked bolt are significant $\mathrm{CR}$ reactive spots.

\section{Secretome validation}

Comparison of the secretome data sets with recent human and rodent adipokine studies revealed several new adipocyte-secreted proteins: 3-hydroxyisobutyryl-CoA hydrolase; cDNA FLJ54471, highly similar to complement C1r subcomponent; cDNA FLJ59142, highly similar to epididymal secretory protein E1; cDNA FLJ60094, highly similar to F-actin capping protein subunit beta; HP protein and ubiquitin carboxyl-terminal hydrolase isozyme L3 $[3,13$, 24-28]. Together, this validation analysis revealed 6 novel identified adipocyte secreted proteins in this study.

To the best of our knowledge this is the first detailed proteomics study of CR-induced secretome changes of in vitro human adipocytes. As such, we compared our data with data from human trial studies in which CR-induced adipokine changes were determined. Klempel [29] and Varady [30] reviewed in vivo studies of CR-induced human adipokine changes, which revealed a relatively small set of plasma circulating adipokines including adiponectin, IL 6, IL-8, leptin, monocyte chemotactic protein-1, resistin, retinol-binding protein and tumor necrosis factor-alpha. From these proteins only adiponectin, interleukin (IL) 6, IL-8, leptin and retinol-binding protein 4 are known as human adipocyte-secreted proteins. All other 
described proteins are secreted from other cells in the WAT. Comparison with our data revealed only adiponectin as a shared protein [29,30].

A

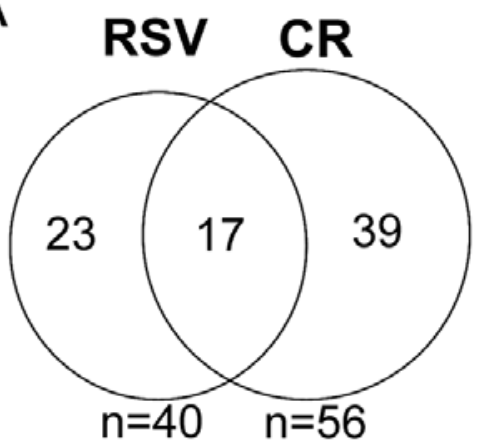

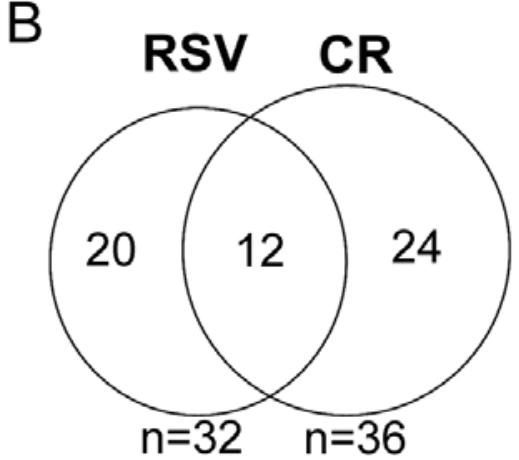

Figure 3: A: Venn diagram showing the comparison of all identified secreted proteins from this $\mathrm{CR}$ study and our previous RSV study ${ }^{13}$. B: Venn diagram of the distribution of identified secreted proteins as single spots from this CR study and the previous RSV study ${ }^{13}$.

\section{Comparison of the secretion profile of CR- and RSV-treated adipocytes}

Since RSV mimics CR we compared the data described in this study with data from our recently published study on RSV-mediated changes in the human adipocyte secretome [13] (Table 3 and Figure 3). From all identified secreted proteins from this CR study 17 proteins were also present in the RSV data (Figure 3A), including adipokines like adiponectin and PAI-1 identified in spots containing more than one protein. To gain more insight in regulation patterns only proteins identified as single protein per spot were compared (Table 3). As such, 12 proteins were identified in the CR as well as RSV data sets. Furthermore, this comparison revealed an additional set of 20 unique proteins from the RSV study and 24 unique proteins from this CR study (Figure 3B and Table 3).

Table 3: Comparison of single spot identifications from secretome of CR and RSV-treated SGBS adipocytes

\begin{tabular}{|c|c|c|c|}
\hline $\begin{array}{c}\text { Accession } \\
\text { number }\end{array}$ & Protein name & $\begin{array}{c}\text { This study } \\
\text { ID }\end{array}$ & $\begin{array}{c}\text { RSV study }^{13} \\
\text { ID }\end{array}$ \\
\hline \multicolumn{4}{|c|}{$\begin{array}{l}\text { classical secreted } \\
\text { extracellular matrix }\end{array}$} \\
\hline P27797 & Calreticulin & & 2 \\
\hline P02452 & Collagen alpha-1(I) chain & 37 & 17 \\
\hline P02461 & Collagen alpha-1(III) chain & 30 & 6 \\
\hline P20908 & Collagen alpha-1(V) chain & 2 & \\
\hline P12109 & Collagen alpha-1(VI) chain & 28 & 26 \\
\hline P08123 & Collagen alpha-2(I) chain & 40 & 57 \\
\hline P08572 & Collagen alpha-2(IV) chain & 87 & \\
\hline P12110 & Collagen alpha-2(VI) chain & 53 & 31 \\
\hline Q12805 & EGF-containing fibulin-like extracellular matrix protein 1 & & 12 \\
\hline P02751 & Fibronectin & 27 & 27 \\
\hline P11047 & Laminin subunit gamma-1 & & 4 \\
\hline Q14112 & Nidogen-2 & & 3 \\
\hline \multicolumn{4}{|c|}{ regulation/signaling } \\
\hline P08253 & $78 \mathrm{kDa}$ glucose-regulated protein & & 15 \\
\hline Q15848 & Adiponectin & & 9 \\
\hline P02649 & Apolipoprotein $\mathrm{E}$ & 8 & 8 \\
\hline D6QS48 & Calumenin & 6 & \\
\hline $\mathrm{P} 14625$ & Endoplasmin & 12 & \\
\hline
\end{tabular}


Table 3: Comparison of single spot identifications from secretome of CR and RSV-treated SGBS adipocytes

\begin{tabular}{|c|c|c|c|}
\hline $\begin{array}{c}\text { Accession } \\
\text { number }\end{array}$ & Protein name & $\begin{array}{l}\text { This study } \\
\text { ID }\end{array}$ & $\begin{array}{l}\text { RSV study } \\
\text { ID }\end{array}$ \\
\hline \multicolumn{4}{|c|}{ classical secreted } \\
\hline P09382 & Galectin-1 & & 5 \\
\hline P06396 & Gelsolin & 52 & \\
\hline P00738 & Haptoglobin & 38 & 30 \\
\hline Q02818 & Nucleobindin-1 & & 14 \\
\hline P36955 & Pigment epithelium-derived factor & 23 & 33 \\
\hline P05121 & Plasminogen activator inhibitor 1 & & 38 \\
\hline P41222 & Prostaglandin-H2 D-isomerase & 16 & \\
\hline P30101 & Protein disulfide-isomerase $\mathrm{A} 3$ & & 25 \\
\hline P02787 & Serotransferrin & & 41 \\
\hline Q8NBS9 & Thioredoxin & 3 & \\
\hline P25311 & Zinc-alpha-2-glycoprotein & 7 & \\
\hline \multicolumn{4}{|c|}{ immune regulation } \\
\hline B4DPQ0 & $\begin{array}{l}\text { cDNA FLJ54471, highly similar to Complement C1r } \\
\text { subcomponent }\end{array}$ & 29 & \\
\hline P09871 & Complement C1s subcomponent & 14 & \\
\hline A6XNE2 & Complement factor $\mathrm{D}$ & & 45 \\
\hline P06702 & Protein S100-A9 & 51 & \\
\hline \multicolumn{4}{|c|}{ non-classical secreted } \\
\hline Q6NVY1 & 3-hydroxyisobutyryl-CoA hydrolase & 75 & \\
\hline P06733 & Alpha-enolase & 78 & \\
\hline P09496 & Clathrin light chain $\mathrm{A}$ & & 1 \\
\hline P23528 & Cofilin-1 & 72 & \\
\hline Q13011 & Delta(3,5)-Delta(2,4)-dienoyl-CoA isomerase & & 46 \\
\hline P09622 & Dihydrolipoyl dehydrogenase & & 49 \\
\hline P15090 & Fatty acid-binding protein & & 32 \\
\hline P09104 & Gamma-enolase & 10 & \\
\hline P04792 & Heat shock protein beta-1 & 41 & \\
\hline Q6NSB4 & HP protein & 90 & \\
\hline P07195 & L-lactate dehydrogenase $B$ chain & & 37 \\
\hline Q06830 & Peroxiredoxin-1 & & 51 \\
\hline P32119 & Peroxiredoxin-2 & & 19 \\
\hline P30086 & Phosphatidylethanolamine-binding protein 1 & 71 & 43 \\
\hline P28074 & Proteasome subunit beta type- 5 & 84 & \\
\hline P50454 & Serpin $\mathrm{H} 1$ & & 56 \\
\hline P04179 & Superoxide dismutase [Mn] & 57 & \\
\hline P00441 & Superoxide dismutase [Cu-Zn] & & 28 \\
\hline Q01995 & Transgelin & 55 & \\
\hline P15374 & Ubiquitin carboxyl-terminal hydrolase isozyme L3 & 5 & \\
\hline P08670 & Vimentin & 4 & 11 \\
\hline
\end{tabular}

\section{Discussion}

During obesity, excess energy storage results in dysfunctional WAT with concomitant risks for development of metabolic diseases and increased mortality [4]. Changes in lifestyle, especially dietary changes, are known to prevent age and obesity-associated diseases, which results in improved life quality and a longer lifespan [6, 7]. Beneficial effects of CR on circulating adipokines have been demonstrated with human intervention studies. However, a detailed investigation of CR-induced human adipocyte secretome changes has not been 
published yet. Therefore, we analyzed CR-mediated changes in the secretome of human SGBS adipocytes.

For optimal growth conditions cells were cultured in macronutrient-rich medium-containing high glucose levels (DMEM/F12 medium with $17.5 \mathrm{mM}$ glucose). To simulate calorie restriction the similar medium was used with a drastic reduction of the glucose concentration in order to obtain significant TG reduction while cell viability was ensured. As such, the low glucose concentration of $0.5 \mathrm{mM}$ equals $3 \%$ of the high DMEM medium glucose concentration $(17.5 \mathrm{mM})$ and is about $10 \%$ of the normal physiological glucose concentration. With these experimental set up the positive impact of CR on the adipocyte secretion profile is demonstrated. In addition, 6 novel adipocyte-secreted proteins have been identified.

Previously it was shown that RSV positively influences obesity-associated diseases. This has been attributed to the CR-mimicking effects of RSV [9, 15, 31]. Recently we published a study on RSV-mediated changes of the human adipocyte secretome [13]. As such, a comparison of this CR study and the previous RSV study was performed to analyze similarities and differences between both treatments with respect to their effects on the adipocyte secretome.

There are strong indications that the beneficial effects of CR and RSV intervention are mediated via SIRT1 and its down-stream signalling [31-33]. Activation of SIRT1 stimulates fat mobilization in WAT by increased lipolysis activity as well as increased free fatty acids (FFA) release. This results in a reduction of the TG storage [9, 23]. Indeed, RSV [13] as well as CR (this study) resulted in a significant reduction of TG contents of human adipocytes. The intracellular TG reduction by low calorie diets might be coupled to increased intracellular lipolysis as shown before in mice [34]. Here, a CR-induced HSL expression and a -reduced ATGL expression was observed which might indicate a HSL-mediated reduction of the TG content. This is in line with the findings of Ryden et al. [35] but is in contrast to a RSVmediated TG reduction [13]. Adipocytes incubated with RSV demonstrated no ATGL stimulation and a significant HSL down-regulation [13] while Lasa et al. [36] showed an ATGL-mediated lipolysis. This difference in lipolysis induction might be an example of different CR and RSV-induced effects on adipocytes. However, an increased lipolysis and decreased TG content are associated with an increased basal glycerol release which was not measured here. In contrast, a CR-mediated down-regulation of glycerol release was identified. This was also observed when SGBS adipocytes were treated with a low $(5 \mu \mathrm{m})$ RSV concentration [13]. A possible explanation is that reduced intracellular TG stimulates the transcriptional coactivator peroxisome proliferators-activated receptor $\mathrm{Y}$ (PPARY) coactivator

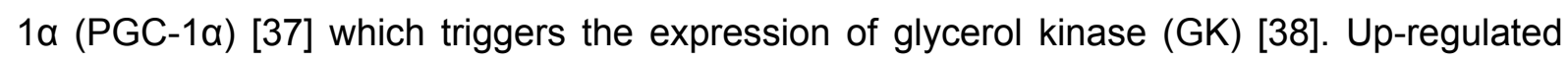




\section{CHAPTER 7}

GK turns glycerol into glycerol-3-phosphate which can be broken down via the glycolysis pathway [39].

As shown here, reduced intracellular TG content leads to cell shrinking which induces a structural remodelling of the adipocytes [2]. Reduced cell size urges a decreased expression of cell structure components. The down-regulation of most of the collagen isoforms and subunits identified here is in line with this. However, a CR-induced secretion of all collagen alpha-2(I) isoforms, various vimentin isoforms and the actin remodelling protein cofilin-1 was also observed. A possible explanation of such divergent regulation pattern might be the fact that collagen type I isoforms are also part of adiposomes, which are adipocyte-specific vesicles involved in the secretion of adipokines [40]. As such, enhanced adiposome secretion may in turn increase the amount of collagen type I in the cell medium. Alternatively, a divergent regulation pattern might also be the result of a slowly CR-mediated cell size reduction and in turn an adaption of the stress-related cell shrinking process in a cell survival manner, which would support the assumption of Mariman and Wang [2]. Compared to RSV, CR seems a less stress-inducing process in relation to intracellular TG reduction. This is reflected by the regulation pattern of structural proteins but also by the fact that most of the stress-associated proteins, including calreticulin, nucleobindin-1, putative heat shock $70 \mathrm{kDa}$ protein 7 and the apoptosis inducing factor galectin-1 [41] were identified within the RSV data set [13]. An explanation for RSV-induced stress-related proteins might be the relative high RSV concentration (75 uM) applied or the in vitro oxidation of RSV which generates $\mathrm{H}_{2} \mathrm{O}_{2}$ and $\mathrm{H}_{2} \mathrm{O}_{2}$-induced stress responses [42]. However, increased $\mathrm{H}_{2} \mathrm{O}_{2}$ levels would result in decreased SIRT levels [43-45] whereas in our previous study a RSV-induced SIRT-1 expression was observed [13] which indicates a normal response.

The investigation of CR-induced changes of the human adipocyte secretome revealed 6 novel adipocyte-secreted proteins. According to UniProtKB 3-hydroxyisobutyryl-CoA hydrolase and ubiquitin carboxyl-terminal hydrolase isozyme $L 3$ are associated with ubiquitin cell regulation, signalling and synthesis processes. cDNA FLJ54471, highly similar to complement C1r subcomponent; cDNA FLJ59142, highly similar to epididymal secretory protein E1; cDNA FLJ60094, highly similar to F-actin capping protein subunit beta and HP are proteins of which the functions have not been established. Their similarities to known proteins might be taken as a lead to understand their real biological function. Since CR is known to introduce beneficial effects towards a less inflammatory phenotype as well as an improvement of obesity-associated metabolic disorders $[31,46]$ their function may be sought in this direction.

In this study adipocyte specific secretion proteins with metabolic functions were detected of which apoE, haptoglobin, PEDF, prostaglandin-H2 D-isomerase and ZAG were identified in a single protein spots and as such their regulation may contribute to a better understanding of 
CR-mediated effects. ApoE regulates TG turnover and expression of genes of the lipid synthesis [47]. In obese mice ApoE is decreased and again increased during CR [48]. In line with an ApoE induction during TG release a RSV-induced ApoE regulation pattern was observed in SGBS adipocytes [13]. In contrast, in this CR study the identified spot containing the full-length ApoE protein was significantly down-regulated. Huang et al. $[47,48]$ described that CR-induced ApoE expression is associated with increased TG mass, TG syntheses and decreased TG hydrolysis as an adipose tissue and energy homeostasis protection response. Similarly, we previously showed an up-regulation of ApoE in mature SGBS adipocytes compared to SGBS preadipocytes [3]. As such, a down-regulation of ApoE in parallel to reduced TG levels in SGBS cells after CR would be in line with such an ApoE-induced energy homeostasis mechanism.

Another interesting protein is the adipokine ZAG which is involved in lipid metabolism as lipid-mobilizing factor and is highly expressed in adipose tissue [49] and several types of tumors [50]. It has been shown that ZAG is down-regulated in obesity and is negatively correlated with plasma insulin, C-reactive protein and monocyte chemotactic protein-1 levels which are all related to obesity-related metabolic disorders [51, 52]. Therefore, CR-induced ZAG expression might promote beneficial effects with respect to the metabolic syndrome. Our CR-induced adipocyte secretome data demonstrated that 3 of the 4 identified single protein ZAG spots were down-regulated while the up-regulated ZAG single protein spot was related to a smaller MW compared to the other 3 ZAG identifications. Reduced MW is mostly associated to protein modifications/fragmentations and in turn inactivity of proteins. However, different studies of secreted ZAG in relation to obesity revealed inconsistent results. Yeung et al. [53] showed increased circulating ZAG levels while Gong et al. [54] and Selva et al. [55] demonstrated decreased circulating ZAG levels in obesity. These contradictive findings may be due to differentially expressed and secreted ZAG isoforms. Our data indeed showed different secreted ZAG isoforms which differ in pl and molecular weight. In addition, a RSVinduced TG release showed no effect on the ZAG secretion [13]. As such, ZAG needs further investigation to understand its part in obesity and obesity-associated disorders.

CR also has been shown to be antioxidative [56] and anti-inflammatory [10, 57]. Furthermore, CR-treated adipocytes secrete adipokines which are related to the obesityassociated metabolic and inflammatory phenotype including haptoglobin, PEDF and prostaglandin-H2 D-isomerase identified in this study. The inflammatory adipokine haptoglobin shows chemotactic activity towards monocytes and is up-regulated during inflammation and obesity [58]. Within this study a CR-mediated down-regulation was observed. Haptoglobin knock-out mice show an improvement of insulin resistance and hepatosteatosis via increased glucose tolerance, insulin sensitivity and increased adiponectin expression [59] As such, CR-mediated down-regulation of haptoglobin appears 


\section{CHAPTER 7}

beneficial for obesity-associated metabolic complications. The association of prostaglandinH2 D-isomerase with obesity and metabolic syndrome is still under debate. Elevated levels of prostaglandin-H2 D-isomerase negatively affect adipogenesis and decrease the risk for glucose intolerance and insulin resistance [60,61]. Here, two isoforms were identified which are significantly up and down-regulated. This might be linked to CR-specific reactions towards an improvement of obesity-associated metabolic status as previously described [60, 61]. The adipokine PEDF is related to insulin resistance and a pro-inflammatory status in obese patients [62, 63]. As such, a CR-mediated down-regulation of PEDF is another argument for the CR-induced positive impact on obesity-associated inflammatory status, insulin resistance, glucose intolerance and WAT dysfunction.

A comparison with the previously described RSV secretome study [13] revealed that both treatment strategies lead to a less inflammatory phenotype and a phenotype reflecting improvement of metabolic complications; even their secretion patterns differ from each other as shown in Figure 3. RSV treatment resulted in reduced PEDF and PAI-1 as well as induced adiponectin and ApoE secretion, which reverse the human adipocyte secretion profile towards a less inflammatory phenotype and a more insulin-sensitizing pattern [63-68]. Except for the reduced PEDF secretion, CR treatment did not clearly demonstrate such protein regulation pattern. Adiponectin and PAI-1 were only identified in multi-protein spots and ApoE was even down-regulated by CR-treatment. In addition, RSV induced secretion of complement factor $D$ which was not observed in this study. Complement factor $D$ induces lipolysis and TG release [69] and as such, it may enhance the strong positive effects of RSV treatment. During obesity complement factor $D$ is defectively expressed which may negatively influence the signalling pathway of the immune-related complement system [70] as well as lipolysis and the TG release. An induced expression of intact complement factor $D$ during obesity might lead to improved immune responses and increased lipolysis activity.

Taken together, the analysis of the RSV and CR data sets indicate that, compared to CR, RSV treatment induces stronger beneficial effects towards improved obesity-associated metabolic complications and a relieved inflammatory phenotype. However, the CR-mediated regulation pattern of haptoglobin, PEDF and prostaglandin-H2 D-isomerase as well as the reduced secretion of stress-related proteins also indicates a clear CR-induced positive impact on obesity-associated disorders which was also shown by several intervention studies including the study of Varady et al. [10]. As such, the differences between CR- and RSVregulated adipocyte secretomes might be due to different cellular signalling responses and regulation mechanisms. This is also reflected by different isoforms of similar proteins which have been found regulated in both studies. This finally results in the idea that CR is less forceful than RSV but appears to reach its positive effects with minor cellular stress. 


\section{Conclusion}

We showed a CR-induced adipocyte TG reduction associated with a change of the adipocyte secretion profile, in which 6 novel adipocyte-secreted proteins were discovered. Changes in the adipocyte-secretion pattern indicate a positive effect of $C R$ with respect to obesityassociated inflammatory phenotype, WAT dysfunction and metabolic status. Compared to RSV the CR-induced cell-shrinking process was associated with a milder cellular stress response and in addition, affected differently the adipocyte secretome with respect to obesity-associated metabolic dysfunctions.

\section{Acknowledgement}

We thank Dr. Johan Jocken, Department Human Biology, Maastricht University, The Netherlands for helpful discussions. Freek Bouwman and Sonja Kallendrusch, Department Human Biology, Maastricht University and Eric Royackers, Biomedical Research Institute, Hasselt University are acknowledged for their practical support.

\section{References}

1. Lehr, S., S. Hartwig, and H. Sell, Adipokines: A treasure trove for the discovery of biomarkers for metabolic disorders. Proteomics Clin Appl, 2012. 6(1-2): p. 91-101.

2. Mariman, E.C. and P. Wang, Adipocyte extracellular matrix composition, dynamics and role in obesity. Cell Mol Life Sci, 2010. 67(8): p. 1277-92.

3. Rosenow, A., et al., Identification of Novel Human Adipocyte Secreted Proteins by Using SGBS Cells. J Proteome Res, 2010. 9: p. 5389-401.

4. Diamanti-Kandarakis, E., et al., The Impact of Endocrine Disruptors on Endocrine Targets. Horm Metab Res, 2010. 42(8): p. 543-52.

5. Dixon, J.B., The effect of obesity on health outcomes. Mol Cell Endocrinol, 2010. 316(2): p. 104-8.

6. McCay, C.M., M.F. Crowell, and L.A. Maynard, The effect of retarded growth upon the length of life span and upon the ultimate body size. 1935. Nutrition, 1989. 5(3): p. 155-71; discussion 172.

7. Lefevre, M., et al., Caloric restriction alone and with exercise improves CVD risk in healthy non-obese individuals. Atherosclerosis, 2009. 203(1): p. 206-13. 


\section{CHAPTER 7}

8. Lin, S.J., P.A. Defossez, and L. Guarente, Requirement of NAD and SIR2 for lifespan extension by calorie restriction in Saccharomyces cerevisiae. Science, 2000. 289(5487): p. 2126-8.

9. Picard, F., et al., Sirt1 promotes fat mobilization in white adipocytes by repressing PPAR-gamma. Nature, 2004. 429(6993): p. 771-6.

10. Varady, K.A., et al., Degree of weight loss required to improve adipokine concentrations and decrease fat cell size in severely obese women. Metabolism, 2009. 58(8): p. 1096-101.

11. Skurk, T., et al., Relationship between adipocyte size and adipokine expression and secretion. J Clin Endocrinol Metab, 2007. 92(3): p. 1023-33.

12. Hu, Y., et al., The controversial links among calorie restriction, SIRT1, and resveratrol. Free Radic Biol Med, 2011. 51(2): p. 250-6.

13. Rosenow, A., et al., Resveratrol-induced changes of the human adipocyte secretion profile. J Proteome Res, 2012. 11(9): p. 4733-43.

14. Olholm, J., et al., Anti-inflammatory effect of resveratrol on adipokine expression and secretion in human adipose tissue explants. Int J Obes (Lond), 2010. 34(10): p. 154653.

15. Timmers, S., et al., Calorie Restriction-like Effects of 30 Days of Resveratrol Supplementation on Energy Metabolism and Metabolic Profile in Obese Humans. Cell Metab, 2011. 14(5): p. 612-22.

16. Howitz, K.T., et al., Small molecule activators of sirtuins extend Saccharomyces cerevisiae lifespan. Nature, 2003. 425(6954): p. 191-6.

17. Wabitsch, M., et al., Characterization of a human preadipocyte cell strain with high capacity for adipose differentiation. Int J Obes Relat Metab Disord, 2001. 25(1): p. 815.

18. Bouwman, F., J. Renes, and E. Mariman, A combination of protein profiling and isotopomer analysis using matrix-assisted laser desorption/ionization-time of flight mass spectrometry reveals an active metabolism of the extracellular matrix of 3T3-L1 adipocytes. Proteomics, 2004. 4(12): p. 3855-63.

19. Dumont, D., et al., Characterization of mature rat oligodendrocytes: a proteomic approach. J Neurochem, 2007. 102(2): p. 562-76.

20. Vizcaino, J.A., et al., The PRoteomics IDEntifications (PRIDE) database and associated tools: status in 2013. Nucleic Acids Res, 2013. 41(Database issue): $p$. D1063-9.

21. Barsnes, H., et al., PRIDE Converter: making proteomics data-sharing easy. Nat Biotechnol, 2009. 27(7): p. 598-9. 
22. Vizcaino, J.A., et al., The Proteomics Identifications database: 2010 update. Nucleic Acids Res, 2010. 38(Database issue): p. D736-42.

23. Wang, R., et al., PRIDE Inspector: a tool to visualize and validate MS proteomics data. Nat Biotechnol, 2012. 30(2): p. 135-7.

24. Schweiger, M., et al., Adipose triglyceride lipase and hormone-sensitive lipase are the major enzymes in adipose tissue triacylglycerol catabolism. J Biol Chem, 2006. 281(52): p. 40236-41.

25. Lehr, S., et al., Identification and validation of novel adipokines released from primary human adipocytes. Mol Cell Proteomics, 2012. 11(1): p. M111 010504.

26. Molina, $\mathrm{H}_{\text {. }}$, et al., Temporal profiling of the adipocyte proteome during differentiation using a five-plex SILAC based strategy. J Proteome Res, 2009. 8(1): p. 48-58.

27. Roelofsen, $\mathrm{H}$., et al., Comparison of isotope-labeled amino acid incorporation rates (CILAIR) provides a quantitative method to study tissue secretomes. Mol Cell Proteomics, 2009. 8(2): p. 316-24.

28. Zhong, J., et al., Temporal profiling of the secretome during adipogenesis in humans. J Proteome Res, 2010. 9(10): p. 5228-38.

29. Zvonic, S., et al., Secretome of primary cultures of human adipose-derived stem cells: modulation of serpins by adipogenesis. Mol Cell Proteomics, 2007. 6(1): p. 18-28.

30. Klempel, M.C. and K.A. Varady, Reliability of leptin, but not adiponectin, as a biomarker for diet-induced weight loss in humans. Nutr Rev, 2011. 69(3): p. 145-54.

31. Varady, K.A. and M.K. Hellerstein, Do calorie restriction or alternate-day fasting regimens modulate adipose tissue physiology in a way that reduces chronic disease risk? Nutr Rev, 2008. 66(6): p. 333-42.

32. Baur, J.A., Resveratrol, sirtuins, and the promise of a DR mimetic, in Mech Ageing Dev. 2010. p. 261-9.

33. Barger, J.L., et al., A low dose of dietary resveratrol partially mimics caloric restriction and retards aging parameters in mice. PLoS One, 2008. 3(6): p. e2264.

34. Smith, J.J., et al., Small molecule activators of SIRT1 replicate signaling pathways triggered by calorie restriction in vivo. BMC Syst Biol, 2009. 3: p. 31.

35. Benz, V., et al., Sexual dimorphic regulation of body weight dynamics and adipose tissue lipolysis. PLoS One, 2012. 7(5): p. e37794.

36. Ryden, M., et al., Comparative studies of the role of hormone-sensitive lipase and adipose triglyceride lipase in human fat cell lipolysis. Am J Physiol Endocrinol Metab, 2007. 292(6): p. E1847-55.

37. Lasa, A., et al., Resveratrol regulates lipolysis via adipose triglyceride lipase. J Nutr Biochem, 2011. 23(4): p. 379-84. 


\section{CHAPTER 7}

38. Chen, Z., J.Y. Norris, and B.N. Finck, Peroxisome proliferator-activated receptorgamma coactivator-1alpha (PGC-1alpha) stimulates VLDL assembly through activation of cell death-inducing DFFA-like effector B (CideB). J Biol Chem, 2010. 285(34): p. 25996-6004.

39. Mazzucotelli, A., et al., The transcriptional coactivator peroxisome proliferator activated receptor (PPAR)gamma coactivator-1 alpha and the nuclear receptor PPAR alpha control the expression of glycerol kinase and metabolism genes independently of PPAR gamma activation in human white adipocytes. Diabetes, 2007. 56(10): p. 2467-75.

40. Harding, J.W., Jr., et al., Proportional activities of glycerol kinase and glycerol 3phosphate dehydrogenase in rat hepatomas. Biochem J, 1975. 148(3): p. 545-50.

41. Aoki, N., et al., Identification and characterization of microvesicles secreted by 3T3L1 adipocytes: redox- and hormone-dependent induction of milk fat globule-epidermal growth factor 8-associated microvesicles. Endocrinology, 2007. 148(8): p. 3850-62.

42. Hsu, D.K., R.Y. Yang, and F.T. Liu, Galectins in apoptosis. Methods Enzymol, 2006. 417: p. 256-73.

43. Yang, N.C., C.H. Lee, and T.Y. Song, Evaluation of resveratrol oxidation in vitro and the crucial role of bicarbonate ions. Biosci Biotechnol Biochem, 2010. 74(1): p. 63-8.

44. Bai, P., et al., PARP-1 inhibition increases mitochondrial metabolism through SIRT1 activation. Cell Metab, 2011. 13(4): p. 461-8.

45. Furukawa, A., et al., $\mathrm{H} 2 \mathrm{O} 2$ accelerates cellular senescence by accumulation of acetylated p53 via decrease in the function of SIRT1 by NAD+ depletion. Cell Physiol Biochem, 2007. 20(1-4): p. 45-54.

46. Mader, I., et al., Identification of a novel proapoptotic function of resveratrol in fat cells: SIRT1-independent sensitization to TRAIL-induced apoptosis. Faseb J, 2010. 24(6): p. 1997-2009.

47. Anderson, R.M. and R. Weindruch, Calorie restriction: progress during mid-2005-mid2006. Exp Gerontol, 2006. 41(12): p. 1247-9.

48. Huang, Z.H., C.A. Reardon, and T. Mazzone, Endogenous ApoE expression modulates adipocyte triglyceride content and turnover. Diabetes, 2006. 55(12): p. 3394-402.

49. Huang, Z.H., et al., Nutritional regulation of adipose tissue apolipoprotein $E$ expression. Am J Physiol Endocrinol Metab, 2007. 293(1): p. E203-9.

50. Bing, C., et al., Zinc-alpha2-glycoprotein, a lipid mobilizing factor, is expressed in adipocytes and is up-regulated in mice with cancer cachexia. Proc Natl Acad Sci U S A, 2004. 101(8): p. 2500-5. 
51. Hassan, M.I., et al., Zinc alpha 2-glycoprotein: a multidisciplinary protein. Mol Cancer Res, 2008. 6(6): p. 892-906.

52. Ceperuelo-Mallafre, $\mathrm{V}$., et al., Circulating and adipose tissue gene expression of zincalpha2-glycoprotein in obesity: its relationship with adipokine and lipolytic gene markers in subcutaneous and visceral fat. J Clin Endocrinol Metab, 2009. 94(12): p. 5062-9.

53. Mracek, T., et al., The adipokine zinc-alpha2-glycoprotein (ZAG) is downregulated with fat mass expansion in obesity. Clin Endocrinol (Oxf), 2010. 72(3): p. 334-41.

54. Yeung, D.C., et al., Serum zinc-alpha2-glycoprotein correlates with adiposity, triglycerides, and the key components of the metabolic syndrome in Chinese subjects. J Clin Endocrinol Metab, 2009. 94(7): p. 2531-6.

55. Gong, F.Y., et al., Zinc-alpha2-glycoprotein is involved in regulation of body weight through inhibition of lipogenic enzymes in adipose tissue. Int J Obes (Lond), 2009. 33(9): p. 1023-30.

56. Selva, D.M., et al., Lower zinc-alpha2-glycoprotein production by adipose tissue and liver in obese patients unrelated to insulin resistance. J Clin Endocrinol Metab, 2009. 94(11): p. 4499-507.

57. Merry, B.J., Oxidative stress and mitochondrial function with aging--the effects of calorie restriction. Aging Cell, 2004. 3(1): p. 7-12.

58. Nunn, A.V., et al., Inflammatory modulation of exercise salience: using hormesis to return to a healthy lifestyle. Nutr Metab (Lond), 2010. 7: p. 87.

59. Maffei, M., et al., The obesity and inflammatory marker haptoglobin attracts monocytes via interaction with chemokine (C-C motif) receptor 2 (CCR2). BMC Biol, 2009. 7: p. 87.

60. Lisi, S., et al., Obesity-associated hepatosteatosis and impairment of glucose homeostasis are attenuated by haptoglobin deficiency. Diabetes, 2011. 60(10): p. 2496-505.

61. Chowdhury, A.A., et al., Sustained expression of lipocalin-type prostaglandin D synthase in the antisense direction positively regulates adipogenesis in cloned cultured preadipocytes. Biochem Biophys Res Commun, 2011. 411(2): p. 287-92.

62. Ragolia, L., et al., Accelerated glucose intolerance, nephropathy, and atherosclerosis in prostaglandin D2 synthase knock-out mice. J Biol Chem, 2005. 280(33): p. 2994655.

63. Borg, M.L., et al., Pigment epithelium-derived factor regulates lipid metabolism via adipose triglyceride lipase. Diabetes, 2011. 60(5): p. 1458-66.

64. Famulla, S., et al., Pigment epithelium-derived factor (PEDF) is one of the most abundant proteins secreted by human adipocytes and induces insulin resistance and 


\section{CHAPTER 7}

inflammatory signaling in muscle and fat cells. Int J Obes (Lond), 2011. 35(6): p. 76272.

65. Mandal, P., et al., The anti-inflammatory effects of adiponectin are mediated via a heme oxygenase-1-dependent pathway in rat Kupffer cells. Hepatology, 2010. 51(4): p. $1420-9$.

66. Baitsch, D., et al., Apolipoprotein $E$ induces antiinflammatory phenotype in macrophages. Arterioscler Thromb Vasc Biol, 2011. 31(5): p. 1160-8.

67. Odrowaz-Sypniewska, G., Markers of pro-inflammatory and pro-thrombotic state in the diagnosis of metabolic syndrome. Adv Med Sci, 2007. 52: p. 246-50.

68. Wang, J.J., et al., Anti-inflammatory effects of pigment epithelium-derived factor in diabetic nephropathy. Am J Physiol Renal Physiol, 2008. 294(5): p. F1166-73.

69. Nieuwdorp, M., et al., Hypercoagulability in the metabolic syndrome. Curr Opin Pharmacol, 2005. 5(2): p. 155-9.

70. Cianflone, K., Z. Xia, and L.Y. Chen, Critical review of acylation-stimulating protein physiology in humans and rodents. Biochim Biophys Acta, 2003. 1609(2): p. 127-43.

71. Rosen, B.S., et al., Adipsin and complement factor D activity: an immune-related defect in obesity. Science, 1989. 244(4911): p. 1483-7. 
Chapter 8

General Discussion 


\section{Introduction}

Obesity and its associated metabolic complications, including chronic inflammatory phenotypes, are a major health and economic burden that has increased constantly during the last decades [1]. Therefore, detailed investigation of obesity and its related metabolic dysfunction is essential to further characterize these complications. In addition, genetic factors that are specific for weight gain, loss and maintenance also play a major role in obesity. To obtain a more comprehensive view on obesity, these should be identified and further examined, too.

A detailed investigation of the adipocyte secretome as shown in the studies described in this thesis is an important step towards a better understanding of functional and biological aspects of adipocytes within the development of obesity, obesity-associated disorders, weight loss and weight loss-related metabolic improvements. Such knowledge is essential to develop promising treatment strategies for obesity and its specific complications.

\section{Cell model}

To study the secretome of preadipocytes and adipocytes within the adipose tissue the use of a valid fat cell model is important. The first adipocyte secretome studies were performed with 3T3-L1 mouse or rat adipocytes [2-6]. Such rodent fat cell models are easy to obtain, to culture and to manipulate. In addition, total adipose tissue samples from human or rodent origin were used to perform similar secretome studies $[7,8]$. Both cell models and whole adipose tissue samples have contributed significantly to the current understanding that adipose tissue is not only the major site for fat storage, but is also an important endocrine organ. As such, many adipokines have been discovered, like for example PEDF [9] and vaspin [10]. However, it has become clear that there are considerable biological differences between rodents and humans, not only on the level of embryonic stem cells [11] and immunology [12], but particularly with respect to the adipocyte secretion profile. As an example, the mouse adipocyte-secreted protein resistin is secreted in the human adipose tissue by macrophages [13].

A whole adipose tissue-related secretome study consists of a mixed secretion profile of all adipose tissue containing cells including adipocytes, preadipocytes, macrophages, and stromal vascular cells. Studying adipose tissue biopsies gives one an idea of the total protein secretion profile but does not distinguish between the cellular origins of the proteins. Therefore, recent secretome studies, including the studies described in this thesis, have focused on the identification of novel human adipokines by using isolated and purified human fat cell material, which indeed revealed a more specific picture of the adipocyte-secreted proteins [14-19]. The availability of primary human cell material to obtain proper human 
adipocytes, especially visceral adipocytes, is limited. In addition, the donor-specific variability of adipose tissue and especially the loss of their differentiation capacity after isolation are points of concern. Therefore, we opted for a human cell strain, which was established from subcutaneous adipose tissue from a Simpson Golabi Behmel Syndrome (SGBS) patient. SGBS cells are valid preadipocytes and have no donor-specific variability. SGBS preadipocytes are able to differentiate up to $90 \%$ over 30 cell generation whereas their biology, morphology and function are similar to primary subcutaneous human (pre)adipocytes [20]. As such, this cell strain is ideal to culture, manipulate and to reveal information about adipocyte development, protein secretion or metabolism. So far, SGBS cells have been used to study adipocyte differentiation [17, 21, 22], apoptosis [23-25], glucose and fatty acid metabolism $[24,26]$ as well as regulation and expression of proteins including adipokines as shown in this thesis and several other studies [27-30]. Such investigations clearly show that human in vitro cell models are necessary to analyze adipocyte specific effects in reaction to particular conditions. As such, possible biological mechanisms related to a human obesity can be discovered. However, with respect to in vitro models the full knowledge of inter-organ cross-talk and substrate action is missing. Therefore, in vivo studies remain essential for a complete (patho)physiological understanding of the adipose tissue biology.

\section{Technical challenges}

The recent research community has not managed yet to identify all adipocyte-secreted proteins. There are several reasons to explain this. Firstly, there is limited availability of human cells and tissues as described above. Secondly, there are technical challenges among the different proteomic-based applications, such as throughput rate, instrument sensitivity, dynamic range of protein masses and abundances and quantification of protein expression. As such, no single proteomics approach can result in a complete protein identification and quantification map of adipocyte-secreted proteins.

Immunoaffinity applications including Western blotting and enzyme-linked immunosorbent assay (ELISA) and antibody arrays are highly efficient methods to detect and quantify proteins or protein modifications. However, the use of protein-specific antibodies makes these approaches relatively resource intensive, time consuming and expensive. In addition, such antibody-mediated approaches exclude the discovery of novel proteins and the simultaneous detection of whole tissue or body fluid protein maps [31-35]. These limitations of immunoaffinity assays necessitated the use of mass spectrometry (MS) technology to discover novel candidates of proteins. An application that received a considerable amount of research effort during the last decades was the 2-dimensional electrophoresis (2-DE) 


\section{CHAPTER 8}

approach [36]. The 2-DE technique has contributed to our understanding of the protein regulation, signaling and modulation of complex biological systems. Until now, 2-DE is still a common and preferred research-driven proteomics technique to perform de novo sequencing and protein identification for organisms with no or an incomplete genome sequence. Furthermore, it allows detection of specific protein modifications and protein isoforms [36]. The major advantages of 2-DE are that it is a relatively simple and a robust technique which is easily implemented with relatively low costs. In a narrow $\mathrm{pH}$ range up to 5000 protein spots can be resolved on one single gel [37]. In addition, down to $1 \mathrm{ng}$ protein per spot can be visible [38]. Nevertheless, the 2-DE approach is not suitable to analyze hydrophobic proteins and proteins above $250 \mathrm{kDa}$ or under $10 \mathrm{kDa}$. The detection of $1000-1500$ protein spots by a broad $\mathrm{pH}$-range gel compared to the many thousands of proteins in a complex sample makes it a challenge to compare different experiments with each other. A low reproduction rate and the time consuming procedure are additional disadvantages of this technique [39, 40].

To overcome such a restricted view on protein expression, shot gun approaches via liquid chromatography (LC) coupled to mass spectrometry (LC-MS) were introduced in proteomics research. Reversed phase, ion exchange or/and size exclusion LC columns allow separation of large amounts of analytes including proteins and peptides. Multidimensional protein identification techniques which use two different LC columns enable a high throughput of protein identifications in complex samples. However, such high throughput requires long analysis times and also includes a high level of false positive identifications. The handling of the dynamic range of protein expression in (complex) samples as well as the quantification of the masses and abundances of identified proteins are still major challenges for LC-MS approaches [41-44]. Stable isotopic labeling techniques (iTRAQ and ICAT) have been introduced for MS-mediated protein quantification [45]. However, although being an established technology, the application of stable isotopes still suffers from high costs and technical difficulties. Furthermore, proper protein quantification depends on labeling efficiencies [46-48]. Therefore, label-free MS-based protein quantification technologies have been developed that gained more and more popularity [46, 49-51]. Such label-free approaches are based on chromatographic peak intensity measurements or spectral counting, which allows the determination of relative protein abundances and in turn increases the protein dynamic range detection [52]. However, a combination of high sensitive, high throughput and cost effective MS-based quantitative protein analysis is still a major issue in modern proteomics [53].

An alternative MS application for precise and quantitative protein analyses of complex biological samples is the selected reaction monitoring (SRM) approach [54]. Within the last 30 years SRM was often used as a reference quantitative technique to analyze small 
molecules $[55,56]$. Its hypothesis-driven character includes the analysis of a defined set of proteins of interest within a complex sample. This eliminates the problem of the high abundant protein dominance and the unpredicted detection/identification of posttranslational modifications (PTMs) as experienced with shot-gun proteomics. As such, this approach is highly selective and sensitive over a wide dynamic protein range [57]. The disadvantages of SRM are based on a complex working approach, which requires several intermediate steps to generate the peptides of interest. In addition, the target analyte and the background material in the sample consist of the same material, i.e. peptides, which may lead to interference and ion suppression. This may result in limited detection capacity and indicates that SRM still needs improvement of the instrument set-up and the quantification strategy [54].

An alternative MS approach for the identification of small and low abundant proteins/molecules is capillary electrophoresis (CE) coupled to MS. The low molecularweight proteome/peptidome is mostly associated with biological active metabolites such as cytokines or hormones. The identification of such clinically relevant molecules is an important issue in understanding the development, progress and curing of diseases or metabolic dysfunctions including obesity. To identify such proteins the SRM technique could be a helpful tool too (see above). However, in contrast to SRM, CE separates small molecules from a complex biological sample and allows the analysis of thousands of polypeptides within 60 min without losing any resolution or compatibility [58]. Compared to LC-MS, CE-MS is highly reproducible, stable and sensitive. In addition, in contrast to LC-MS no buffer gradient is used because the migration of the analytes is controlled via the electric field strength [5860]. Despite this fact, CE is limited with respect to protein size (not above $20 \mathrm{kDa}$ ) and loading capacity (maximum 10-100 nl) [61]. The precise and accurate work character of CEMS makes it possible to identify intact small proteins/peptides, including their PTMs and to distinguish between mass differences of $0.001 \mathrm{Da}$ in the so called top-down experiment. In bottom-up experiments, where proteins are digested first in peptides to gain more information on high-molecular-weight proteomes, small proteins are easily lost and as such information on the low-molecular-weight proteome/peptidome [61].

Each MS application described cannot be considered as the gold standard for the identification and quantification of a complete proteome. This is also the case for the adipocyte proteome/secretome studies as was also shown in chapter 2. However, all these MS applications with their advantages and disadvantages push researchers to think carefully about their research questions and goals. In this respect, we were aware of the bottlenecks of 2-DE. Although the 2-DE technique was a good proteomic approach to analyze and identify known and novel adipocyte secretion proteins, to understand and quantify protein regulation during adipocyte differentiation (chapter 3 ), hypoxic conditions (chapter 5), RSV 


\section{CHAPTER 8}

treatment (chapter 6), CR conditions (chapter 7) as well as to compare CR- and RSVmediated adipocyte secretome changes (chapter 7 ), the identification of specific PTMs, small and low-abundant proteins requires the use of additional LC-MS or CE-MS techniques. Therefore, the gold standard may be a combination of different proteomics techniques to identify and quantify a maximum amount of proteins which would allow a more comprehensive view on the (pre)adipocyte proteome/secretome. This would enhance the possibility to characterize protein regulation, signaling and modification during specific biological set ups of (pre)adipocytes or cells from other tissues and in turn discover new biomarkers specific for metabolic dysfunction or disease.

\section{Adipose tissue as an endocrine organ}

Adipose tissue is known to store energy as triglycerides (TG). Excess fat storage during obesity results in the extension of existing adipocytes (hypertrophy) and in an increase of adipocyte number (hyperplasia) [62, 63]. Since adipose tissue is described as a major endocrine organ [64-66] more and more research groups became interested in adipose tissue-secreted factors. However, the most important feature is the fact that the para- and endocrine cross-talk mediated by adipocyte-secreted factors regulate the energy balance, which includes glucose up-take, fat accumulation, lipid metabolism and lipolysis. As such, several adipose tissue-secreted proteins have been discovered, including adiponectin [67], IL-6 [66, 68], leptin [69] and TNF-a [70]. Today, more than 600 adipocyte-specific secreted proteins have been discovered, which include ECM proteins, hormones, cytokines, immune regulation proteins, neurotrophins, proteins involved in angiogenesis, inflammation, signaling, lipid and glucose metabolism [71]. The ongoing identification of novel adipocyte-secreted proteins might be an indication of a stepwise solving of the technical limitations, including the introduction of valid human fat cell models, improved cell culture protocols and improved sensitivity of applied proteomics methods. However, the total cellular proteome contains an estimated number of 500.000 to 1.000 .000 different proteins from which $10-15 \%$ is predicted as secretory proteins [72] which indicates that (many) more adipocyte-secreted proteins are to be discovered. In addition, manipulation of adipocytes leads to different protein regulation and in turn a different protein secretion profile, which will even enhance the number of potential protein identifications. As such, we have demonstrated that (pre)adipocyte manipulation by differentiation stimulation, hypoxia, resveratrol treatment and calorie restriction leads to different secretion profiles in which we identified 23 novel adipocyte secreted proteins. 


\section{Secretion pattern determine WAT function}

As shown in this thesis manipulation of (pre)adipocytes leads to changed (pre)adipocyte secretion profiles. As such, dependent on the intervention, differentially regulated adipocyte secretion proteins can result on one hand in improvements of the obesity-associated metabolic syndrome and inflammatory phenotype (chapter 6 and 7 ) and on the other hand in (pre)adipocyte dysregulation and dysfunction (chapter 3, 4 and 5). Analyses of the positive as well as negative protein regulation and expression pathways are essential to understand the WAT function in the context of obesity and its associated diseases. The investigation of secretome changes during adipocyte differentiation, presented in chapter 3 and in several different adipocyte proteomic studies [2-6, 14, 19, 73-75] , demonstrated a clear difference between preadipocyte and adipocyte secretion patterns, which covers proteins involved in extracellular matrix (ECM) adaptation, regulation and signaling, processing as well as immune regulation. The most dramatic secretome changes during differentiation, which is due to structure remodeling, were obtained for the ECM proteins. We identified ECM protein including the collagen alpha-1 (I) and alpha 2 (I) chains as well as beta ig-h3 in several different spots which were differently intense and expressed. In addition, posttranslational modifications of these proteins, such as the $\mathrm{C}$-terminal peptides were identified. The presence of such C-terminal peptides as well as the identification of processing proteins like procollagen C-endopeptidase enhancer 1 and metalloproteinase inhibitor 1 [76] indicates a preadipocyte differentiation-dependent turnover of ECM proteins. It also indicates a complex regulation of the ECM proteins by posttranslational modification in preadipocytes and adipocytes. As such, inhibition of ECM proteins to try and limit obesity-associated enhanced WAT growth might be a powerful treatment strategy but can also be too complicated and too damaging for the biological function of other tissue/organs in an interactive regulation system.

Another example of preadipocyte and adipocyte differences is shown in chapter 4. Tissue factor (TF) is primarily known for its function in the initiation of the coagulation cascade. Its function within adipose tissue is mostly associated with the development of a hypercoagulable state, which may contribute to obesity-associated cardiovascular complications [77]. An up-regulation might negatively influence human health. Here, we analyzed 8-fold higher TF activity in adipocytes compared to preadipocytes. This might be due to higher density of anionic phospholipids in the exoleaflet of adipocytes $[78,79]$ or to increase cell surface size of adipocytes compared to preadipocytes [80] In contrast, compared to adipocyte-derived medium, an increased level of active TF was measured in preadipocyte-derived medium. The measurement of a secreted form of TF might be explained by the existence of 2 different TF's: a cell-bound TF (aa 1 - 295) and a TF lacking the transmembrane domain (aa $252-274$ ), described as the soluble/secreted alternatively 


\section{CHAPTER 8}

spliced (asTF) [81]. More recently, Censarek et al. [82] have demonstrated that asTF has no factor $\mathrm{X}$ and thrombin generation activity. This may explain the low TF activity in adipocyte medium compared to a higher cell-bound activity.

As described TF is primarily known as coagulation factor and is associated with obesityassociated cardiovascular complications [77]. Down regulation of TF expression could be interesting to treat TF-induced health problems including hypercoagulation [83], atherothrombotic disease [84], obesity-induced inflammation and in turn insulin resistance [85]. As such, HMG-CoA reductase inhibitors (statins) which reduce TF expression [86] would be a possible treatment strategy. We found that treatment of human (pre)adipocytes with simvastatin in a pharmacologically relevant dose reduced TF activity with about $50 \%$. However, treatment of obesity-associated hypercoagulation via inhibition of TF signaling could also result in the inhibition of angiogenesis and vascularization. Under hypoxic conditions adipose tissue TF can contribute to angiogenic processes [87-89], which in turn promotes vascular endothelial cell proliferation and new vessel formation [91]. Furthermore, the alternatively spliced/secreted form promotes the process of angiogenesis instead of the coagulation cascade by activating factor $X$ [90]. Furthermore, the alternatively spliced/secreted form is mentioned to promote the process of angiogenesis instead of the coagulation cascade by activating factor $X$ [91]. Therefore, TF inhibition may result in negative systemic effects associated with dysfunction of white adipose tissue (WAT) such as an increased level of inflammation and fibrosis [92].

An alternative strategy to treat obesity-associated complications is the circumvention of hypoxia within fast growing obesity-associated WAT. The hypothesis of hypoxic conditions within enlarged adipose tissue is based on the fact that the blood flow to adipose tissue is not elevated in obese subjects compared to lean subjects $[93,94]$ and that the diameter of hypertrophic adipocytes in obese subjects is increased $[80,95]$. Together this results in a poorly vascularised and in turn an $\mathrm{O}_{2}$-insufficient adipose tissue [96-98]. As such, cells of the adipose tissue switch from an oxidative phosphorylation to an anaerobic energy metabolism [99]. An anaerobic state leads to changes in lipid metabolism [100, 101], differentiation process [102] and secretion behavior [98, 103]. In chapter 5, we examined alterations in the adipocyte secretion profile under conditions mimicking hypoxia. In this study most of the identified secreted proteins were down-regulated. In addition, the up-regulation of secreted collagens by adipocytes, thought to be essential for cell survival, and the down-regulation of secreted collagens by preadipocytes, known to disturb the differentiation process, indicated an ECM dysregulation of both cell types. Other hypoxia studies also demonstrated differentially expressed ECM proteins, which resulted in adipose tissue fibrosis and in turn lead to increased adipose dysfunction and insulin resistance [104, 105]. As such, enlarged and/or hypoxic adipocytes lead to WAT dysfunction and eventually to obesity-associated 
metabolic complications. One way to overcome hypoxia is to stimulate angiogenesis in WAT. However, such stimulation should be limited to the WAT organ since enhanced angiogenesis within different organs/tissues might stimulate tumor growth [106]. This again demonstrates the complex intra- and intercellular regulation mechanisms of biological systems like organs and tissues.

\section{Treatment strategies of obesity and its metabolic complications}

Strategies to treat obese patients are bariatric surgery, dietary intervention, increased physical activity and pharmacotherapy [107-109]. Although bariatric surgery is the most risky one, it provides considerable weight loss and maintenance success. It alleviates insulin resistance in overweight type 2 diabetes patients, reduces hypertension and hyperlipidemia, improves life quality and as such decreases obesity-associated mortality. In addition, bariatric surgery reduces the number of medications as well as the health care costs [110]. There are three types of surgery: the restriction (a diminished calorie intake by reducing the stomach volume), the malabsorptive (a reduction of the intestinal absorption of nutrition by shortening the functional small bowel length) and the mixed procedure (a combination of restriction and malabsorptive surgery). The restriction procedure is characterized by less surgical risks and less metabolic complications but results in lower weight reduction. Compared to the restriction procedure the malabsorptive surgery results in a greater weight loss but also shows a higher perioperative morbidity risk as well as a greater potential for late postoperative metabolic complications. The third method is most frequently used but includes apart from benefits also the risks of the malabsorptive procedure. The surgical risks, the metabolic complications as well as the high surgery costs and limited surgical expertise still prevent bariatric surgery from being a universal treatment for obesity $[107,108]$.

Pharmacotherapy, physical activity and a diet are non-invasive interventions. There are several drugs available with weight reduction effects. The disadvantages of a pharmacotherapy are the short-term effects on weight loss, which necessitates a life long treatment for weight maintenance with increased risks for side effects like depression and headaches [109, 111-113]. A promising treatment supplement could be RSV which is shown to mimic positive effects of calorie restriction in obese humans including improved insulin resistance, decreased levels of blood glucose, TG and cytokines as well as increased intramyocellular lipid levels, reduced intrahepatic lipid content and systolic blood pressure $[114,115]$. As shown in chapter 6 and 7 we demonstrated that RSV treatment of mature human adipocytes in vitro positively affects the adipocyte secretome. This may have beneficial health effects including improved insulin sensitivity and anti-inflammatory effects. However, the applied $75 \mu \mathrm{M}$ RSV is a high concentration compared to physiological RSV 
concentrations which are not achieved by normal diets or measured in the blood plasma $[114,116,117]$. The bioavailability of RSV in in vivo studies is dramatically reduced due to a rapid reaction of resveratrol with glucuronides and sulfates [118]. Therefore, pharmacological intervention with high RSV concentrations might be obligatory to obtain the RSV-induced beneficial effects. Nevertheless, Timmers et al. [114] demonstrated that physiological RSV levels continued for 30 days exert beneficial health effects in humans. However, compared to in vitro studies RSV has a limited life span in vivo. In addition, it has been demonstrated that $\mathrm{RSV}$ is already oxidized after $24 \mathrm{~h}$ in vitro with a considerable decrease in its activity. As such, to achieve similar effects in a shorter intervention period higher concentrations seem to be required. For the in vitro RSV study in chapter 6 an intervention period of $48 \mathrm{~h}$ was used which was ideal to obtain a sufficient amount of secreted proteins although the risk of oxidized RSV and in turn the production of oxidative stress by generating $\mathrm{H}_{2} \mathrm{O}_{2}$ [119] was present. Increased $\mathrm{H}_{2} \mathrm{O}_{2}$ levels were shown to decrease SIRT1 expression [120-123]. In contrast, SIRT1 is thought to be indirectly activated by RSV via Epac1 and AMPK signaling pathway [124]. In our hands, functional RSV was demonstrated by Western blotting analysis which revealed a significant RSV-induced SIRT1 expression. The 48h intervention study in chapter 6 showed RSV-mediated changes in the adipocyte adipokine profile which reflected improvement of obesity-induced metabolic complications and anti-inflammatory processes.

Several studies revealed that physical activity alone during a short period of time as noninvasive obesity treatment strategy had no effect on weight loss because of reduced resting energy expenditure and/or life style habits [125-127]. Still, the advantage of physical activity is the improvement of cardiovascular conditions, insulin sensitivity and glycemic control, while a long-term physical activity does show an improved weight management [125-127]. A dietary intervention often results in a short-term weight reduction followed by weight regain after cessation of the diet. Still, in chapter 7 we showed that calorie restriction results in reduced intracellular TG content and secretome changes reflecting an improved obesityassociated metabolism. The reduced intracellular TG content resulted in cell shrinking and structural remodeling which normally is associated with decreased expression of cell structure components. However, CR induced the secretion for ECM proteins which might be due to a divergent regulation pattern [2] and/or to an adaption of the stress-related cell shrinking process in a cell survival manner. This may be related to the slower cell size reduction compared to RSV treatment. It seems that $C R$ is a less stress-inducing process in relation to intracellular TG reduction when compared to RSV treatment. However, the CRinduced beneficial effects were also shown in human intervention studies which revealed improved circulating adipokine patterns already with a $5 \%$ weight reduction [128]. These improvements are attributed to the decreased adipocyte size since adipokine expression and adipocyte size are correlated [80]. In addition, the reviews of human intervention studies of 
Klempel and Varady [129] and Varady and Hellerstein [130] indeed revealed CR-induced changes of human adipokine secretion patterns that included adiponectin, IL 6, IL-8, leptin, monocyte chemotactic protein-1, resistin, retinol-binding protein and TNF- $\alpha$. However, the effect of CR on adipocyte-secreted adipokines remained unclear. In chapter 7 it is shown that CR changes the secretion of adipokines like haptoglobin [131, 132], PEDF [133, 134] and prostaglandin-H2 D-isomerase $[135,136]$ in a way that obesity-associated metabolic complications and inflammatory phenotype would be improved. As such, CR may have a positive impact on obesity-associated inflammatory status, insulin resistance, glucose intolerance and WAT dysfunction. Although $\mathrm{CR}$ and RSV resulted in TG reduction, comparison of $\mathrm{CR}$ and RSV-mediated changes in adipokine patterns revealed different regulation patterns. RSV treatment resulted in a more affected adipokine secretion profile with a stronger effect on stress-related proteins (Chapter 7 ). This finding resulted in the idea that CR is less forceful than RSV but appears to reach its positive effects with minor cellular stress. Both treatment strategies appear to regulate different cellular signaling mechanisms with respect to improvement of obesity-associated metabolic dysfunctions and inflammatory phenotypes.

The fact that weight regain is often observed after an intervention with $C R$, pharmacological/supplemental treatment, or physical activity, may be due to our environment, lifestyle and habits. However, there might be an alternative biological explanation. Reduced adipocyte cell size urges the remodeling of the ECM. As such, cell shrinking leads to cellular stress and may eventually induce apoptosis [76, 137]. To overcome cellular stress and cellular apoptosis shrinking adipocytes might secrete signaling proteins to the brain that leads to increased food intake and as such stimulates the so-called yo-yo effect [137]. However, pharmacotherapy or dietary intervention/physical activity that is combined with behavior therapy has shown to be most successful in weight maintenance after weight loss [109]. In addition, several studies have shown that genetic factors are crucial for gaining weight [138-140]. Such genetic factors are also crucial to loose and maintain weight [141]. Studies have shown that such factors are mostly individually expressed, regulated and secreted. Such individual effects comply with the study of Wadden et al. [109] involving individual dietary treatments. As such, the adipose tissue as an interacting organ is subjected to general biological as well as individual mechanisms.

\section{Conclusion}

This thesis confirms the importance of proteomics and protein investigation in the field of obesity research. Although there are still technical obstacles we were able to demonstrate that the (pre)adipocyte secretome is related to crucial molecular mechanisms and is dynamic 


\section{CHAPTER 8}

by way of protein expression, stimulation, regulation, signaling and inhibition. This dynamic system can be easily manipulated and influenced which is likely useful for understanding the functions of WAT related to normal weight and/or obesity. This understanding is also essential to develop successful prevention/treatment strategies for obesity and its complications.

\section{Suggestions for future research}

Based on the results from this thesis it is shown that the adipokine profile can be regulated by different interventions that may have profound effects on obesity-associated complications. Still, the ideal treatment for obesity and its complications has to be discovered. This might have several reasons. The main reason is that the current results of obesity-associated research, especially the proteomics-related investigations, are still in the beginning of the discovery of the total WAT proteome and of the detailed adipokine regulation patterns in relation to complex biological mechanisms. By using 2-DE part of the (pre)adipocyte adipokine profiles could be covered. Future application of high sensitive LCMS approaches will provide a deeper coverage of the adipokine profiles and also allows the use of less sample material due to increased sensitivity of the technology. This in turn would make a more reliable analysis of primary human visceral and subcutaneous (pre)adipocytes possible. These cells are normally limited in their differentiation capacity, which reduces the amount of secreted proteins that can be analyzed.

Another advantage of high sensitive LC-MS approaches is the identification of low abundant proteins. This technique could achieve direct protein analyses of human samples related to interventions. It would provide the opportunity to study the individual effects and factors related to weight gain, maintenance and reduction. However, a complicating factor is that blood, tissue or urinary samples contain high abundant proteins which make detailed proteome analysis of low abundant proteins nearly impossible. In addition, isolation of fat cells from human tissues is associated with additional cell culturing procedures which would result in manipulation or deletion of the intervention-related effects. Therefore, novel sophisticated sample preparation procedures, together with high sensitive LC-MS approaches combined with reliable and reproducible quantification software would be a promising strategy to enhance the knowledge of protein regulation and expression mechanisms in (pre)adipocytes, WAT and obesity. 


\section{References}

1. Dixon, J.B., The effect of obesity on health outcomes. Mol Cell Endocrinol, 2010. 316(2): p. 104-8.

2. Aoki, N., et al., Identification and characterization of microvesicles secreted by 3T3L1 adipocytes: redox- and hormone-dependent induction of milk fat globule-epidermal growth factor 8-associated microvesicles. Endocrinology, 2007. 148(8): p. 3850-62.

3. Chen, X., et al., Quantitative proteomic analysis of the secretory proteins from rat adipose cells using a 2D liquid chromatography-MS/MS approach. J Proteome Res, 2005. 4(2): p. 570-7.

4. Kratchmarova, I., et al., A proteomic approach for identification of secreted proteins during the differentiation of 3T3-L1 preadipocytes to adipocytes. Mol Cell Proteomics, 2002. 1(3): p. 213-22.

5. Molina, H., et al., Temporal profiling of the adipocyte proteome during differentiation using a five-plex SILAC based strategy. J Proteome Res, 2009. 8(1): p. 48-58.

6. Wang, P., et al., Profiling of the secreted proteins during 3T3-L1 adipocyte differentiation leads to the identification of novel adipokines. Cell Mol Life Sci, 2004. 61(18): p. 2405-17.

7. Celis, J.E., et al., Identification of extracellular and intracellular signaling components of the mammary adipose tissue and its interstitial fluid in high risk breast cancer patients: toward dissecting the molecular circuitry of epithelial-adipocyte stromal cell interactions. Mol Cell Proteomics, 2005. 4(4): p. 492-522.

8. Roca-Rivada, A., et al., Secretome analysis of rat adipose tissues shows locationspecific roles for each depot type. J Proteomics, 2011. 74(7): p. 1068-79.

9. Wang, P., et al., Plasma pigment epithelium-derived factor is positively associated with obesity in Caucasian subjects, in particular with the visceral fat depot. Eur $\mathrm{J}$ Endocrinol, 2008. 159(6): p. 713-8.

10. Li, Q., et al., A novel adipocytokine, visceral adipose tissue-derived serine protease inhibitor (vaspin), and obesity. J Int Med Res, 2008. 36(4): p. 625-9.

11. Ginis, I., et al., Differences between human and mouse embryonic stem cells. Dev Biol, 2004. 269(2): p. 360-80.

12. Mestas, J. and C.C. Hughes, Of mice and not men: differences between mouse and human immunology. J Immunol, 2004. 172(5): p. 2731-8.

13. Arner, P., Resistin: yet another adipokine tells us that men are not mice. Diabetologia, 2005. 48(11): p. 2203-5.

14. Chiellini, C., et al., Characterization of human mesenchymal stem cell secretome at early steps of adipocyte and osteoblast differentiation. BMC Mol Biol, 2008. 9: p. 26. 
15. Kim, J., et al., Comparative analysis of the secretory proteome of human adipose stromal vascular fraction cells during adipogenesis. Proteomics, 2010. 10(3): p. 394405.

16. Lehr, S., et al., Identification and validation of novel adipokines released from primary human adipocytes. Mol Cell Proteomics, 2012. 11(1): p. M111 010504.

17. Rosenow, A., et al., Identification of Novel Human Adipocyte Secreted Proteins by Using SGBS Cells. J Proteome Res, 2010. 9: p. 5389-401.

18. Zhong, J., et al., Temporal profiling of the secretome during adipogenesis in humans. J Proteome Res, 2010. 9(10): p. 5228-38.

19. Zvonic, S., et al., Secretome of primary cultures of human adipose-derived stem cells: modulation of serpins by adipogenesis. Mol Cell Proteomics, 2007. 6(1): p. 18-28.

20. Wabitsch, M., et al., Characterization of a human preadipocyte cell strain with high capacity for adipose differentiation. Int J Obes Relat Metab Disord, 2001. 25(1): p. 815.

21. Newell, F.S., et al., Characterization of the transcriptional and functional effects of fibroblast growth factor-1 on human preadipocyte differentiation. Faseb J, 2006. 20(14): p. 2615-7.

22. Zandbergen, F., et al., The G0/G1 switch gene 2 is a novel PPAR target gene. Biochem J, 2005. 392(Pt 2): p. 313-24.

23. Fischer-Posovszky, P., et al., Conjugated linoleic acids promote human fat cell apoptosis. Horm Metab Res, 2007. 39(3): p. 186-91.

24. Grigem, S., et al., The effect of the HIV protease inhibitor ritonavir on proliferation, differentiation, lipogenesis, gene expression and apoptosis of human preadipocytes and adipocytes. Horm Metab Res, 2005. 37(10): p. 602-9.

25. Fischer-Posovszky, P., et al., Inhibition of death-receptor mediated apoptosis in human adipocytes by the insulin-like growth factor I (IGF-I)/IGF-I receptor autocrine circuit. Endocrinology, 2004. 145(4): p. 1849-59.

26. Lasa, A., et al., Resveratrol regulates lipolysis via adipose triglyceride lipase. J Nutr Biochem, 2011. 23(4): p. 379-84.

27. Do, M.S., et al., Inflammatory gene expression patterns revealed by DNA microarray analysis in TNF-alpha-treated SGBS human adipocytes. Yonsei Med J, 2006. 47(5): p. 729-36.

28. Bodles, A.M., et al., Pioglitazone increases secretion of high-molecular-weight adiponectin from adipocytes. Am J Physiol Endocrinol Metab, 2006. 291(5): p. E1100-5.

29. Bao, Y., et al., Zinc-alpha2-glycoprotein, a lipid mobilizing factor, is expressed and secreted by human (SGBS) adipocytes. FEBS Lett, 2005. 579(1): p. 41-7. 
30. Laumen, H., T. Skurk, and H. Hauner, The HMG-CoA reductase inhibitor rosuvastatin inhibits plasminogen activator inhibitor-1 expression and secretion in human adipocytes. Atherosclerosis, 2008. 196(2): p. 565-73.

31. de Wildt, R.M., et al., Antibody arrays for high-throughput screening of antibodyantigen interactions. Nat Biotechnol, 2000. 18(9): p. 989-94.

32. Kingsmore, S.F., Multiplexed protein measurement: technologies and applications of protein and antibody arrays. Nat Rev Drug Discov, 2006. 5(4): p. 310-20.

33. Kononen, J., et al., Tissue microarrays for high-throughput molecular profiling of tumor specimens. Nat Med, 1998. 4(7): p. 844-7.

34. Nishizuka, S., et al., Proteomic profiling of the NCl-60 cancer cell lines using new high-density reverse-phase lysate microarrays. Proc Natl Acad Sci U S A, 2003. 100(24): p. 14229-34.

35. Paweletz, C.P., et al., Reverse phase protein microarrays which capture disease progression show activation of pro-survival pathways at the cancer invasion front. Oncogene, 2001. 20(16): p. 1981-9.

36. Rogowska-Wrzesinska, A., et al., 2D gels still have a niche in proteomics. J Proteomics, 2013.

37. Fey, S.J. and P.M. Larsen, 2D or not 2D. Two-dimensional gel electrophoresis. Curr Opin Chem Biol, 2001. 5(1): p. 26-33.

38. Smith, R., Two-dimensional electrophoresis: an overview. Methods Mol Biol, 2009. 519: p. 1-16.

39. Lane, C.S., Mass spectrometry-based proteomics in the life sciences. Cell Mol Life Sci, 2005. 62(7-8): p. 848-69.

40. Canas, B., et al., Mass spectrometry technologies for proteomics. Brief Funct Genomic Proteomic, 2006. 4(4): p. 295-320.

41. McDonald, W.H. and J.R. Yates, 3rd, Shotgun proteomics and biomarker discovery. Dis Markers, 2002. 18(2): p. 99-105.

42. Washburn, M.P., et al., Analysis of quantitative proteomic data generated via multidimensional protein identification technology. Anal Chem, 2002. 74(7): p. 16507.

43. Washburn, M.P., D. Wolters, and J.R. Yates, 3rd, Large-scale analysis of the yeast proteome by multidimensional protein identification technology. Nat Biotechnol, 2001. 19(3): p. 242-7.

44. Wu, C.C., et al., A method for the comprehensive proteomic analysis of membrane proteins. Nat Biotechnol, 2003. 21(5): p. 532-8.

45. Gygi, S.P., et al., Quantitative analysis of complex protein mixtures using isotopecoded affinity tags. Nat Biotechnol, 1999. 17(10): p. 994-9. 
46. Sakai, J., et al., 180-labeling quantitative proteomics using an ion trap mass spectrometer. Proteomics, 2005. 5(1): p. 16-23.

47. Wu, W.W., et al., Comparative study of three proteomic quantitative methods, DIGE, cICAT, and ITRAQ, using 2D gel- or LC-MALDI TOF/TOF. J Proteome Res, 2006. 5(3): p. 651-8.

48. Colzani, M., et al., Relative protein quantification by isobaric SILAC with immonium ion splitting (ISIS). Mol Cell Proteomics, 2008. 7(5): p. 927-37.

49. Ono, M., et al., Label-free quantitative proteomics using large peptide data sets generated by nanoflow liquid chromatography and mass spectrometry. Mol Cell Proteomics, 2006. 5(7): p. 1338-47.

50. Wang, G., et al., Label-free protein quantification using LC-coupled ion trap or FT mass spectrometry: Reproducibility, linearity, and application with complex proteomes. J Proteome Res, 2006. 5(5): p. 1214-23.

51. Zhang, B., et al., Detecting differential and correlated protein expression in label-free shotgun proteomics. J Proteome Res, 2006. 5(11): p. 2909-18.

52. Bantscheff, M., et al., Quantitative mass spectrometry in proteomics: a critical review. Anal Bioanal Chem, 2007. 389(4): p. 1017-31.

53. Wang, M., et al., Label-free mass spectrometry-based protein quantification technologies in proteomic analysis. Brief Funct Genomic Proteomic, 2008. 7(5): p. 329-39.

54. Gallien, S., E. Duriez, and B. Domon, Selected reaction monitoring applied to proteomics. J Mass Spectrom, 2011. 46(3): p. 298-312.

55. Hoke, S.H., 2nd, et al., Increasing bioanalytical throughput using pcSFC-MS/MS: 10 minutes per 96-well plate. Anal Chem, 2001. 73(13): p. 3083-8.

56. Kostiainen, R., et al., Liquid chromatography/atmospheric pressure ionization-mass spectrometry in drug metabolism studies. J Mass Spectrom, 2003. 38(4): p. 357-72.

57. Lange, V., et al., Targeted quantitative analysis of Streptococcus pyogenes virulence factors by multiple reaction monitoring. Mol Cell Proteomics, 2008. 7(8): p. 1489-500.

58. Gaspar, A., et al., Trends in CE-MS 2005-2006. Electrophoresis, 2008. 29(1): p. 6679.

59. Frommberger, M., et al., Peptidomic analysis of rat urine using capillary electrophoresis coupled to mass spectrometry. Proteomics Clin Appl, 2007. 1(7): p. 650-60.

60. Kolch, W., et al., Capillary electrophoresis-mass spectrometry as a powerful tool in clinical diagnosis and biomarker discovery. Mass Spectrom Rev, 2005. 24(6): p. 95977. 
61. Metzger, J., et al., Adapting mass spectrometry-based platforms for clinical proteomics applications: The capillary electrophoresis coupled mass spectrometry paradigm. Crit Rev Clin Lab Sci, 2009. 46(3): p. 129-52.

62. Diamanti-Kandarakis, E., et al., The Impact of Endocrine Disruptors on Endocrine Targets. Horm Metab Res, 2010. 42(8): p. 543-52.

63. Gregoire, F.M., Adipocyte differentiation: from fibroblast to endocrine cell. Exp Biol Med (Maywood), 2001. 226(11): p. 997-1002.

64. Siiteri, P.K., Adipose tissue as a source of hormones. Am J Clin Nutr, 1987. 45(1 Suppl): p. 277-82.

65. Smith, S.R., The endocrinology of obesity. Endocrinol Metab Clin North Am, 1996. 25(4): p. 921-42.

66. Mohamed-Ali, V., J.H. Pinkney, and S.W. Coppack, Adipose tissue as an endocrine and paracrine organ. Int J Obes Relat Metab Disord, 1998. 22(12): p. 1145-58.

67. Kadowaki, T., et al., Adiponectin and adiponectin receptors in insulin resistance, diabetes, and the metabolic syndrome. J Clin Invest, 2006. 116(7): p. 1784-92.

68. Mohamed-Ali, V., et al., Subcutaneous adipose tissue releases interleukin-6, but not tumor necrosis factor-alpha, in vivo. J Clin Endocrinol Metab, 1997. 82(12): p. 4196200.

69. Zhang, Y., et al., Mechanisms for LEPR-mediated regulation of leptin expression in brown and white adipocytes in rat pups. Physiol Genomics, 2001. 4(3): p. 189-99.

70. Hotamisligil, G.S., Mechanisms of TNF-alpha-induced insulin resistance. Exp Clin Endocrinol Diabetes, 1999. 107(2): p. 119-25.

71. Lehr, S., S. Hartwig, and H. Sell, Adipokines: A treasure trove for the discovery of biomarkers for metabolic disorders. Proteomics Clin Appl, 2012. 6(1-2): p. 91-101.

72. Liu, J. and B. Rost, Comparing function and structure between entire proteomes. Protein Sci, 2001. 10(10): p. 1970-9.

73. Alvarez-Llamas, G., et al., Characterization of the human visceral adipose tissue secretome. Mol Cell Proteomics, 2007. 6(4): p. 589-600.

74. Roelofsen, $\mathrm{H}_{\text {., }}$ et al., Comparison of isotope-labeled amino acid incorporation rates (CILAIR) provides a quantitative method to study tissue secretomes. Mol Cell Proteomics, 2009. 8(2): p. 316-24.

75. Lim, J.M., et al., Defining the regulated secreted proteome of rodent adipocytes upon the induction of insulin resistance. J Proteome Res, 2008. 7(3): p. 1251-63.

76. Mariman, E.C. and P. Wang, Adipocyte extracellular matrix composition, dynamics and role in obesity. Cell Mol Life Sci, 2010. 67(8): p. 1277-92.

77. Samad, F., M. Pandey, and D.J. Loskutoff, Tissue factor gene expression in the adipose tissues of obese mice. Proc Natl Acad Sci U S A, 1998. 95(13): p. 7591-6. 
78. Bach, R.R., Tissue factor encryption. Arterioscler Thromb Vasc Biol, 2006. 26(3): p. 456-61.

79. Shaw, A.W., et al., The local phospholipid environment modulates the activation of blood clotting. J Biol Chem, 2007. 282(9): p. 6556-63.

80. Skurk, T., et al., Relationship between adipocyte size and adipokine expression and secretion. J Clin Endocrinol Metab, 2007. 92(3): p. 1023-33.

81. Bogdanov, V.Y., et al., Alternatively spliced human tissue factor: a circulating, soluble, thrombogenic protein. Nat Med, 2003. 9(4): p. 458-62.

82. Censarek, P., et al., Alternatively spliced human tissue factor (asHTF) is not procoagulant. Thromb Haemost, 2007. 97(1): p. 11-4.

83. Li, J.Q., et al., Atorvastatin reduces tissue factor expression in adipose tissue of atherosclerotic rabbits. Int J Cardiol, 2007. 115(2): p. 229-34.

84. Mobarrez, F., et al., Atorvastatin reduces thrombin generation and expression of tissue factor, P-selectin and GPIIla on platelet-derived microparticles in patients with peripheral arterial occlusive disease. Thromb Haemost, 2011. 106(2): p. 344-52.

85. Badeanlou, L., et al., Tissue factor-protease-activated receptor 2 signaling promotes diet-induced obesity and adipose inflammation. Nat Med, 2011. 17(11): p. 1490-7.

86. Brandes, R.P., et al., Withdrawal of cerivastatin induces monocyte chemoattractant protein 1 and tissue factor expression in cultured vascular smooth muscle cells. Arterioscler Thromb Vasc Biol, 2003. 23(10): p. 1794-800.

87. Bluff, J.E., et al., Tissue factor, angiogenesis and tumour progression. Breast Cancer Res, 2008. 10(2): p. 204.

88. Belting, M., J. Ahamed, and W. Ruf, Signaling of the tissue factor coagulation pathway in angiogenesis and cancer. Arterioscler Thromb Vasc Biol, 2005. 25(8): p. 1545-50.

89. Liu, Y. and B.M. Mueller, Protease-activated receptor-2 regulates vascular endothelial growth factor expression in MDA-MB-231 cells via MAPK pathways. Biochem Biophys Res Commun, 2006. 344(4): p. 1263-70.

90. Vona-Davis, L. and D.P. Rose, Angiogenesis, adipokines and breast cancer. Cytokine Growth Factor Rev, 2009. 20(3): p. 193-201.

91. Signaevsky, M., et al., Role of alternatively spliced tissue factor in pancreatic cancer growth and angiogenesis. Semin Thromb Hemost, 2008. 34(2): p. 161-9.

92. Rutkowski, J.M., K.E. Davis, and P.E. Scherer, Mechanisms of obesity and related pathologies: the macro- and microcirculation of adipose tissue. Febs J, 2009. 276(20): p. 5738-46. 
93. Virtanen, K.A., et al., Glucose uptake and perfusion in subcutaneous and visceral adipose tissue during insulin stimulation in nonobese and obese humans. J Clin Endocrinol Metab, 2002. 87(8): p. 3902-10.

94. Karpe, F., et al., Impaired postprandial adipose tissue blood flow response is related to aspects of insulin sensitivity. Diabetes, 2002. 51(8): p. 2467-73.

95. Lolmede, K., et al., Effects of hypoxia on the expression of proangiogenic factors in differentiated 3T3-F442A adipocytes. Int J Obes Relat Metab Disord, 2003. 27(10): p. 1187-95.

96. Pasarica, M., et al., Reduced Oxygenation in Human Obese Adipose Tissue Is Associated with Impaired Insulin Suppression of Lipolysis. J Clin Endocrinol Metab, 2010. 95(8): p. 4052-5.

97. Fleischmann, E., et al., Tissue oxygenation in obese and non-obese patients during laparoscopy. Obes Surg, 2005. 15(6): p. 813-9.

98. Trayhurn, P. and I.S. Wood, Adipokines: inflammation and the pleiotropic role of white adipose tissue. Br J Nutr, 2004. 92(3): p. 347-55.

99. Denko, N.C., Hypoxia, HIF1 and glucose metabolism in the solid tumour. Nat Rev Cancer, 2008. 8(9): p. 705-13.

100. Krishnan, J., et al., Activation of a HIF1alpha-PPARgamma axis underlies the integration of glycolytic and lipid anabolic pathways in pathologic cardiac hypertrophy. Cell Metab, 2009. 9(6): p. 512-24.

101. Yin, J., et al., Role of hypoxia in obesity-induced disorders of glucose and lipid metabolism in adipose tissue. Am J Physiol Endocrinol Metab, 2009. 296(2): p. E33342.

102. Trayhurn, P., B. Wang, and I.S. Wood, Hypoxia in adipose tissue: a basis for the dysregulation of tissue function in obesity? Br J Nutr, 2008. 100(2): p. 227-35.

103. Dimitriadis, G., et al., Impaired postprandial blood flow in adipose tissue may be an early marker of insulin resistance in type 2 diabetes. Diabetes Care, 2007. 30(12): p. 3128-30.

104. Halberg, N., et al., Hypoxia-inducible factor 1alpha induces fibrosis and insulin resistance in white adipose tissue. Mol Cell Biol, 2009. 29(16): p. 4467-83.

105. Khan, T., et al., Metabolic dysregulation and adipose tissue fibrosis: role of collagen VI. Mol Cell Biol, 2009. 29(6): p. 1575-91.

106. Ruf, W., N. Yokota, and F. Schaffner, Tissue factor in cancer progression and angiogenesis. Thromb Res, 2010. 125 Suppl 2: p. S36-8.

107. Hussain, S.S. and S.R. Bloom, The pharmacological treatment and management of obesity. Postgrad Med, 2011. 123(1): p. 34-44. 
108. McEwen, L.N., et al., The cost, quality of life impact, and cost-utility of bariatric surgery in a managed care population. Obes Surg, 2010. 20(7): p. 919-28.

109. Wadden, T.A., M.L. Butryn, and C. Wilson, Lifestyle modification for the management of obesity. Gastroenterology, 2007. 132(6): p. 2226-38.

110. Sjostrom, L., Bariatric surgery and reduction in morbidity and mortality: experiences from the SOS study. Int J Obes (Lond), 2008. 32 Suppl 7: p. S93-7.

111. Davidson, M.H., et al., Weight control and risk factor reduction in obese subjects treated for 2 years with orlistat: a randomized controlled trial. Jama, 1999. 281(3): p. 235-42.

112. James, W.P., et al., Effect of sibutramine on cardiovascular outcomes in overweight and obese subjects. N Engl J Med, 2010. 363(10): p. 905-17.

113. Sakane, N., Pharmacology in health foods:merits and demerits of food with health claims for the prevention of metabolic syndrome. J Pharmacol Sci, 2011. 115(4): p. 476-80.

114. Timmers, S., et al., Calorie Restriction-like Effects of 30 Days of Resveratrol Supplementation on Energy Metabolism and Metabolic Profile in Obese Humans. Cell Metab, 2011. 14(5): p. 612-22.

115. Olholm, J., et al., Anti-inflammatory effect of resveratrol on adipokine expression and secretion in human adipose tissue explants. Int J Obes (Lond), 2010. 34(10): p. 154653.

116. Baur, J.A., et al., Resveratrol improves health and survival of mice on a high-calorie diet. Nature, 2006. 444(7117): p. 337-42.

117. Lagouge, M., et al., Resveratrol improves mitochondrial function and protects against metabolic disease by activating SIRT1 and PGC-1alpha. Cell, 2006. 127(6): p. 110922.

118. Wenzel, E. and V. Somoza, Metabolism and bioavailability of trans-resveratrol. Mol Nutr Food Res, 2005. 49(5): p. 472-81.

119. Yang, N.C., C.H. Lee, and T.Y. Song, Evaluation of resveratrol oxidation in vitro and the crucial role of bicarbonate ions. Biosci Biotechnol Biochem, 2010. 74(1): p. 63-8.

120. Rayalam, S., et al., Resveratrol induces apoptosis and inhibits adipogenesis in 3T3L1 adipocytes. Phytother Res, 2008. 22(10): p. 1367-71.

121. Bai, S.W., et al., Identification and characterization of a set of conserved and new regulators of cytoskeletal organization, cell morphology and migration. BMC Biol, 2011. 9: p. 54.

122. Mader, I., et al., Identification of a novel proapoptotic function of resveratrol in fat cells: SIRT1-independent sensitization to TRAIL-induced apoptosis. Faseb J, 2010. 24(6): p. 1997-2009. 
123. Furukawa, A., et al., $\mathrm{H} 2 \mathrm{O} 2$ accelerates cellular senescence by accumulation of acetylated p53 via decrease in the function of SIRT1 by NAD+ depletion. Cell Physiol Biochem, 2007. 20(1-4): p. 45-54.

124. Park, S.J., et al., Resveratrol ameliorates aging-related metabolic phenotypes by inhibiting cAMP phosphodiesterases. Cell, 2012. 148(3): p. 421-33.

125. Jeffery, R.W., et al., Physical activity and weight loss: does prescribing higher physical activity goals improve outcome? Am J Clin Nutr, 2003. 78(4): p. 684-9.

126. Tumiati, R., et al., Home-centred physical fitness programme in morbidly obese individuals: a randomized controlled trial. Clin Rehabil, 2008. 22(10-11): p. 940-50.

127. Wing, R.R. and J.O. Hill, Successful weight loss maintenance. Annu Rev Nutr, 2001. 21: p. 323-41.

128. Varady, K.A., et al., Degree of weight loss required to improve adipokine concentrations and decrease fat cell size in severely obese women. Metabolism, 2009. 58(8): p. 1096-101.

129. Klempel, M.C. and K.A. Varady, Reliability of leptin, but not adiponectin, as a biomarker for diet-induced weight loss in humans. Nutr Rev, 2011. 69(3): p. 145-54.

130. Varady, K.A. and M.K. Hellerstein, Do calorie restriction or alternate-day fasting regimens modulate adipose tissue physiology in a way that reduces chronic disease risk? Nutr Rev, 2008. 66(6): p. 333-42.

131. Maffei, M., et al., The obesity and inflammatory marker haptoglobin attracts monocytes via interaction with chemokine (C-C motif) receptor 2 (CCR2). BMC Biol, 2009. 7: p. 87.

132. Lisi, S., et al., Obesity-associated hepatosteatosis and impairment of glucose homeostasis are attenuated by haptoglobin deficiency. Diabetes, 2011. 60(10): p. 2496-505.

133. Borg, M.L., et al., Pigment epithelium-derived factor regulates lipid metabolism via adipose triglyceride lipase. Diabetes, 2011. 60(5): p. 1458-66.

134. Famulla, S., et al., Pigment epithelium-derived factor (PEDF) is one of the most abundant proteins secreted by human adipocytes and induces insulin resistance and inflammatory signaling in muscle and fat cells. Int J Obes (Lond), 2011. 35(6): p. 76272.

135. Chowdhury, A.A., et al., Sustained expression of lipocalin-type prostaglandin D synthase in the antisense direction positively regulates adipogenesis in cloned cultured preadipocytes. Biochem Biophys Res Commun, 2011. 411(2): p. 287-92.

136. Ragolia, L., et al., Accelerated glucose intolerance, nephropathy, and atherosclerosis in prostaglandin D2 synthase knock-out mice. J Biol Chem, 2005. 280(33): p. 2994655. 
137. Mariman, E.C., An adipobiological model for weight regain after weight Loss. Adipobiology, 2011. 3.

138. Farooqi, I.S. and S. O'Rahilly, Genetic factors in human obesity. Obes Rev, 2007. 8 Suppl 1: p. 37-40.

139. Rankinen, T., et al., The human obesity gene map: the 2005 update. Obesity (Silver Spring), 2006. 14(4): p. 529-644.

140. Sharma, A.M. and R. Padwal, Obesity is a sign - over-eating is a symptom: an aetiological framework for the assessment and management of obesity. Obes Rev, 2010. 11(5): p. 362-70.

141. Wang, P., et al., Blood profile of proteins and steroid hormones predicts weight change after weight loss with interactions of dietary protein level and glycemic index. PLoS One, 2011. 6(2): p. e16773. 
Summary 


\section{Summary}

Obesity and the obesity-associated metabolic dysfunctions are a major health and economic burden. Adipocyte-secreted proteins play an important role in obesity-associated disorders. Therefore it is essential to study the secreted factors of human (pre)adipocytes, not only to understand the underlying mechanisms of obesity-induced metabolic complications, but also to gain more insight in the regulation of weight gain, weight loss, weight re-gain and weight maintenance. This may aid the development of successful treatment strategies for obesity and its related complications.

In vivo studies are usually confined to plasma and other body fluids and as such the results demonstrate the effect of an intervention but not the underlying molecular mechanisms. That is why in vitro studies of human (pre)adipocytes and adipose tissue are necessary. Technical issues of proteomics technologies, including false positive identification, limited throughput, low sensitivity and quantification still do not allow identifying the complete (pre)adipocyte secretome. However, improved MS approaches developed during the last 10 years have helped to increase the understanding of the adipose tissue/adipocyte proteome and its regulation, including the adipocyte-secreted proteins. Nevertheless, much is still unknown about the molecular mechanisms responsible for the development of obesity and its metabolic complications, as well as strategies to improve the obesity-induced metabolic phenotype. This thesis describes in vitro studies with human SGBS (pre)adipocytes which were investigated regarding the regulation of (pre)adipocyte-specific secreted proteins in the context of triglyceride (TG) accumulation/excess, adipocyte differentiation and TG reduction/increased lipolysis.

Chapter 2 evaluates relevant proteomic technologies in the field of adipocyte biology. In this context new findings, new molecular aspects of adipocyte biology and the discovery of novel adipokines within the adipocyte research field were presented and discussed. They have led to the conclusion that a combination of analysis techniques is essential to cover the identification of the total (pre)adipocyte proteome as well as changes therein.

Secretion differences of SGBS (pre)adipocytes during adipogenesis are described in chapter 3. Secretome profiling was performed on both cell types which led to the identification of 6 novel human adipocyte-secreted proteins. In addition, this study revealed 20 proteins that had not been detected before in human adipose material. In addition, 23 proteins that were previously detected in visceral adipose tissue were found to be secreted by SGBS-cells of subcutaneous origin. Furthermore, this secretome study confirmed SGBS cells as an ideal human (pre)adipocyte cell strain for adipocyte-related proteomics studies. 
Tissue factor (TF) is involved in blood coagulation. Consequently, altered expression of TF during adipose tissue expansion might contribute to the development of obesity-induced thrombosis. As such, we investigated possible TF expression by SGBS (pre)adipocytes, which is reported in chapter 4 . On both cell types and in the medium TF-mediated factor Xa and thrombin activation was observed. Our results indicate that human (pre)adipocytes express functional TF, which may have implications for obesity-induced thrombosis but also for extravascular fibrosis and angiogenesis.

Increased TG accumulation leads to increased adipose tissue mass, which might result in hypoxic conditions. Hypoxic areas have been observed in WAT of obese persons. This may lead to WAT dysregulation and development of obesity-associated metabolic dysregulation. In chapter 5 we describe that $\mathrm{CoCl}_{2}$-induced hypoxic secretome changes of (pre)adipocytes are mostly associated with protein down-regulation and a dysregulation of the extracellular matrix. This confirmed the hypothesis that hypoxia induces detrimental effects within enlarged adipose tissue of obese subjects. Such dysregulation was reflected by an upregulation of collagens in adipocytes which indicated a cell survival process. In preadipocytes collagens were down-regulated which might be indicative for a disturbed differentiation process. In addition, we identified 9 novel (pre)adipocyte secreted proteins of which 6 were regulated by hypoxia.

Treatment strategies for obesity and obesity-induced complications are essential to cope with their associated medical problems. As such, investigation of promising drugs or food supplements is a key feature of obesity research. Since resveratrol (RSV) is known to mimic beneficial health effects of calorie restriction more and more research is performed to understand its biological mechanism. The study in chapter 6 reveals an RSV-mediated increase of intracellular lipolysis, which resulted in a beneficial change of the adipocyte secretion profile. As such, ECM proteins were down-regulated while process-related proteins were mostly up-regulated. In addition, the secretion of proteins that are protective against cellular stress and of proteins involved in the regulation of apoptosis were up-regulated. Furthermore, RSV induced an up-regulation of adiponectin and ApoE and a down-regulation of PAI-1 and PEDF secretion. This indicated a positive impact on the adipocyte-secretome towards an improved obesity-associated inflammatory phenotype and metabolic dysfunction. In addition, 2 novel RSV-regulated adipocyte-secreted proteins were identified.

Another treatment strategy of obesity is a calorie restricted diet. An in vitro effect of CR on the human adipocyte secretion profile had not been investigated before. As such, chapter 7 describes the identification of 6 novel adipocyte-secreted proteins that are regulated by $C R$. 
In addition, it was shown that CR-induced adipocyte TG reduction led to a positively affected adipokine secretion pattern indicative for an improved inflammatory phenotype and an improved obesity-associated metabolic dysfunction including insulin resistance and glucose intolerance.

To distinguish between advantages and disadvantages of an RSV versus a calorie restricted intervention a comparison of the secretion profile of both obesity treatment strategies was also performed and described in chapter 7. CR and RSV differently affected the SGBS adipocyte secretion profile in which both interventions show an improvement of the obesityassociated inflammatory phenotype and the obesity-associated metabolic disorders while CR seems less forceful but appears to reach its positive effects with minor cellular stress.

All these studies were related to the investigation of the human (pre)adipocyte secretome. In chapter 8 the results have been critically discussed in the context of the advantages and disadvantages of the used human cell model and the applied proteomics techniques. It is argued that technical improvement of analysis methods together with the acceptance of individual biological responses might increase the understanding of the endocrine function of the adipose tissue. This may aid the further development of improved obesity treatment strategies. 
Supplemental data 


\section{Supplemental data}

\section{Supplemental data chapter 3}

Supplement 1 of 2 is shown at the website of Journal of Proteomic Research (http://pubs.acs.org/doi/suppl/10.1021/pr100621g).

\section{Supplemental data chapter 5}

Supplement 1

Supplemental Table 1 is a summary of MS/MS-related data of all identified proteins of SGBS adipocytes including spot number, protein name, peptide information and molecular weight. Further detailed information is deposited on the PRIDE server (project accession number: 28672-28710). In addition, statistical calculations (student T-test) are shown for all differentially expressed spots from the $2 \mathrm{D}$ gels.

This Excel table is shown at https://www.dropbox.com/sh/=mas5n7hfdscaxo/WVB5_52Tso as Supplemental data Chapter 5_Supplement 1. 


\section{Adipocytes}
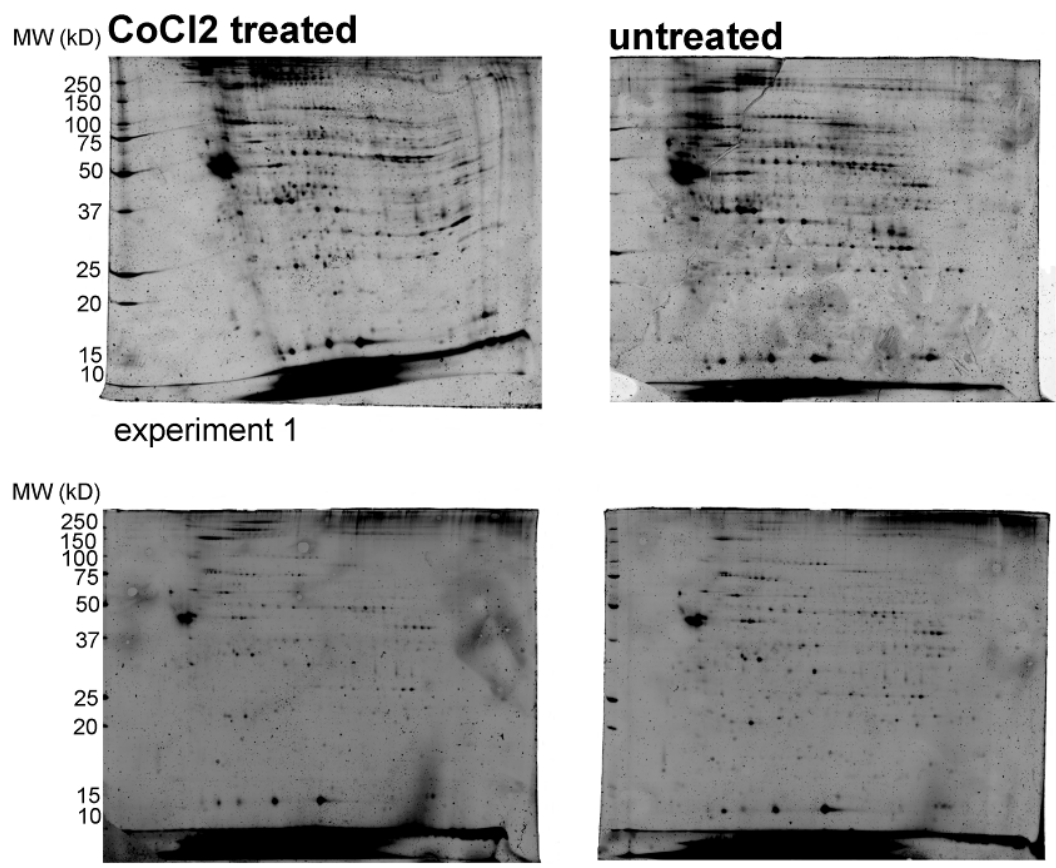

experiment 2
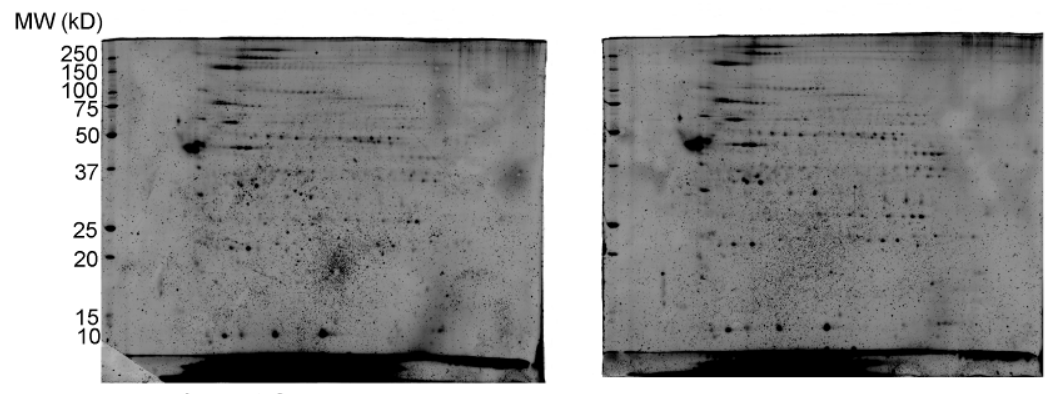

experiment 3
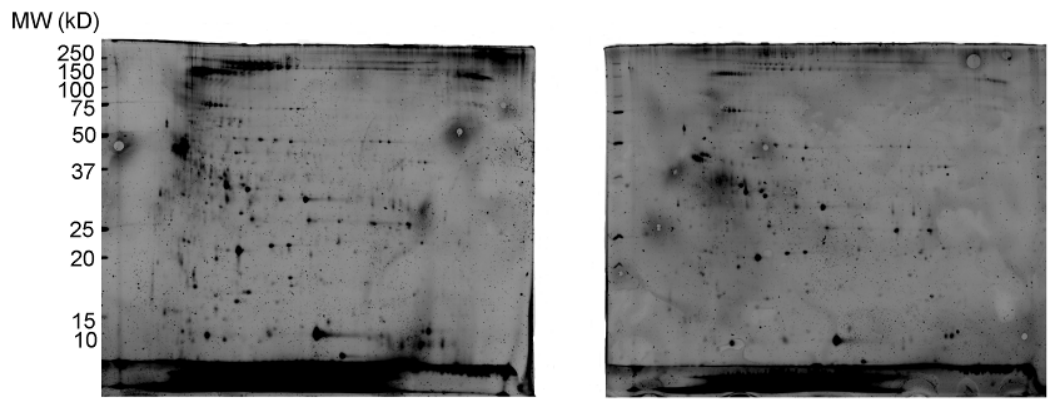

experiment 4

Figure of Supplement 1: 2-D gels of 4 independent experiments for adipocytes

\section{Supplement 2}

Supplemental Table 2 is a summary of MS/MS-related data of all identified proteins of SGBS preadipocytes including spot number, protein name, peptide information and molecular weight. Further detailed information is deposited on the PRIDE server (project accession 
number: 28672-28710). In addition, statistical calculations (student T-test) are shown for all differentially expressed spots from the $2 \mathrm{D}$ gels.

This Excel table is shown at https://www.dropbox.com/sh/0mas5n7hfdscaxo/WVB5_52Tso as Supplemental data Chapter 5_Supplement 2.

\section{Preadipocytes}
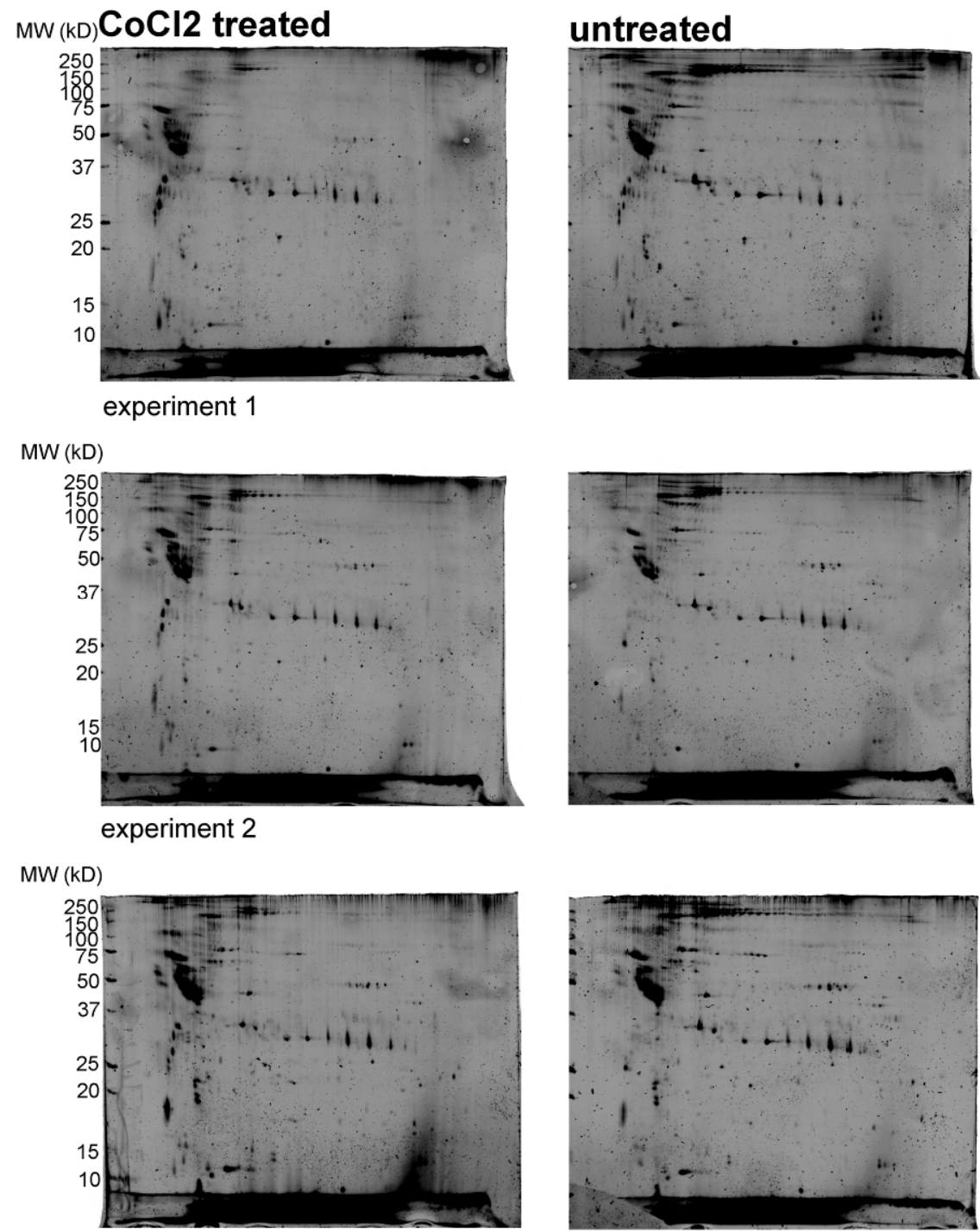

experiment 3

Figure of Supplement 2: 2-D gels of 3 independent experiments for preadipocytes 


\section{Supplement 3}

Supplemental Table 3 is a summary of MS/MS-related data of all identified proteins of SGBS preadipocytes including spot number, protein name, peptide information and molecular weight. Further detailed information is deposited on the PRIDE server (project accession number: 28672-28710). In addition, statistical calculations (student T-test) are shown for all differentially expressed spots from the $2 \mathrm{D}$ gels.

This Excel table is shown at the websit of Journal BBA http://dx.doi.org/10.1016/j.bbapap.2013.10.003.

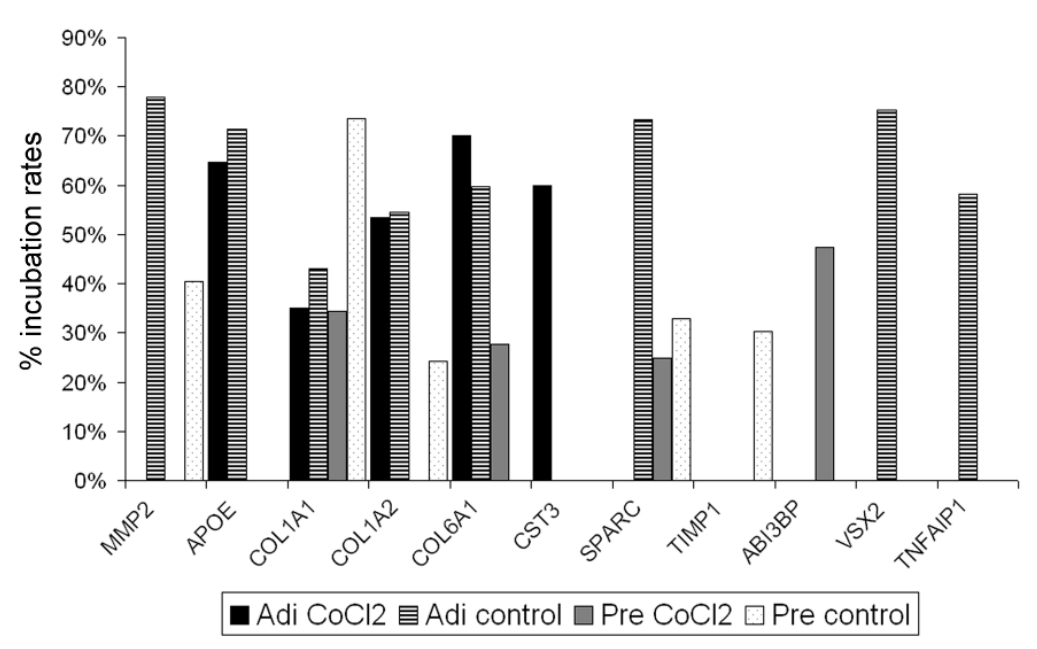

Incorporation rates of stable isotope labeled phenylalanine (L-(ring$2,3,4,5,6{ }^{2} \mathrm{H}_{5}$ ) phenylalanine) in secreted proteins from $\mathrm{CoCl}_{2-}$ treated and non-treated (pre)adipocytes.

Table of Supplement 3: Overview of the identified proteins derived from the protein turnover experiments

\begin{tabular}{|c|c|c|c|c|c|c|c|c|}
\hline $\begin{array}{l}\text { Accession } \\
\text { number }\end{array}$ & protein name & $\begin{array}{l}\text { Protein } \\
\text { score }\end{array}$ & $\begin{array}{c}\text { Protein } \\
\text { score } \\
\text { C. I. \% } \\
\end{array}$ & $\begin{array}{c}\text { unlabeled } \\
\text { petide } \\
\text { mass }\end{array}$ & intensity & $\begin{array}{l}\text { labeled } \\
\text { peptide }\end{array}$ & intensity $\mathrm{F}^{*}$ & differences \\
\hline \multicolumn{9}{|c|}{$\begin{array}{l}\text { Adipocytes } \mathrm{CoCl} 2 \\
\text { classical secreted }\end{array}$} \\
\hline \multirow[t]{3}{*}{ P02649 } & $\begin{array}{l}\text { Apolipoprotein } \\
\text { E precursor }\end{array}$ & 99 & 100 & 1033.5 & $3.7 \mathrm{E}+04$ & 1038.5 & $2.6 \mathrm{E}+04$ & $68.57 \%$ \\
\hline & & & & 1536.6 & $5.4 \mathrm{E}+03$ & 1541.7 & $2.4 \mathrm{E}+03$ & $45.19 \%$ \\
\hline & & & & 1552.7 & $6.2 E+04$ & 1557.7 & $4.5 E+04$ & $72.67 \%$ \\
\hline P02649 & $\begin{array}{l}\text { Apolipoprotein } \\
\text { E precursor }\end{array}$ & 90 & 99.999 & 1033.5 & $4.6 \mathrm{E}+03$ & 1038.5 & $2.6 \mathrm{E}+03$ & $56.02 \%$ \\
\hline \multirow[t]{2}{*}{ P02649 } & $\begin{array}{l}\text { Apolipoprotein } \\
\text { E precursor }\end{array}$ & 91 & 99.999 & 1033.5 & $8.3 E+04$ & 1038.5 & $4.8 E+04$ & $57.53 \%$ \\
\hline & & & & 1552.7 & $1.4 \mathrm{E}+04$ & 1557.7 & $1.2 \mathrm{E}+04$ & $84.92 \%$ \\
\hline \multirow[t]{2}{*}{ P02649 } & $\begin{array}{l}\text { Apolipoprotein } \\
\text { E precursor }\end{array}$ & 66 & 99.704 & 1033.5 & $2.3 E+04$ & 1038.5 & $1.2 \mathrm{E}+04$ & $53.25 \%$ \\
\hline & & & & 1552.7 & $5.8 \mathrm{E}+03$ & 1557.7 & $4.6 E+03$ & $79.67 \%$ \\
\hline \multirow[t]{2}{*}{ P02452 } & $\begin{array}{l}\text { Collagen } \\
\text { alpha } 1(I) \\
\text { chain } \\
\text { precursor }\end{array}$ & 67 & 99.76 & 2086.0 & $2.9 \mathrm{E}+03$ & \multicolumn{2}{|c|}{ no labelling } & \\
\hline & & & & 2657.2 & 4.7E+03 & \multicolumn{2}{|c|}{ no labelling } & \\
\hline P02452 & $\begin{array}{l}\text { Collagen } \\
\text { alpha } 1(\mathrm{I}) \\
\text { chain } \\
\text { precursor }\end{array}$ & 54 & 95.727 & 1030.5 & $4.6 \mathrm{E}+04$ & 1035.5 & $1.6 \mathrm{E}+04$ & $35.15 \%$ \\
\hline P08123 & $\begin{array}{l}\text { Collagen } \\
\text { alpha 2(I) } \\
\text { chain } \\
\text { precursor }\end{array}$ & 60 & 98.796 & 1461.8 & $1.2 \mathrm{E}+04$ & 1466.8 & $6.8 \mathrm{E}+03$ & $56.76 \%$ \\
\hline
\end{tabular}


Table of Supplement 3: Overview of the identified proteins derived from the protein turnover experiments

\begin{tabular}{|c|c|c|c|c|c|c|c|c|}
\hline $\begin{array}{c}\text { Accession } \\
\text { number }\end{array}$ & protein name & $\begin{array}{l}\text { Protein } \\
\text { score }\end{array}$ & $\begin{array}{l}\text { Protein } \\
\text { score } \\
\text { C. I. } \%\end{array}$ & $\begin{array}{l}\text { unlabeled } \\
\text { petide } \\
\text { mass }\end{array}$ & intensity & $\begin{array}{l}\text { labeled } \\
\text { peptide }\end{array}$ & intensity $\mathrm{F}^{*}$ & differences \\
\hline \multicolumn{9}{|c|}{$\begin{array}{l}\text { Adipocytes } \mathrm{CoCl} 2 \\
\text { classical secreted }\end{array}$} \\
\hline \multirow[t]{2}{*}{ P08123 } & $\begin{array}{l}\text { Collagen } \\
\text { alpha 2(I) } \\
\text { chain } \\
\text { precursor }\end{array}$ & 187 & 100 & 1461.7 & $1.1 \mathrm{E}+05$ & 1466.7 & $5.8 \mathrm{E}+04$ & $50.49 \%$ \\
\hline & & & & 1517.7 & $2.9 E+04$ & 1522.7 & $1.5 \mathrm{E}+04$ & $52.89 \%$ \\
\hline \multirow[t]{2}{*}{ P01034 } & $\begin{array}{l}\text { Cystatin C } \\
\text { precursor }\end{array}$ & 55 & 96.279 & 1226.6 & $1.3 E+04$ & 1231.6 & $8.1 E+03$ & $61.66 \%$ \\
\hline & & & & 2060.9 & $3.9 \mathrm{E}+04$ & 2065.9 & $2.3 E+04$ & $58.30 \%$ \\
\hline \multicolumn{9}{|c|}{ non-classical secreted } \\
\hline \multirow[t]{4}{*}{ P08670 } & Vimentin & 112 & 100 & 1093.5 & 1.7E+04 & \multicolumn{2}{|c|}{ no labelling } & \\
\hline & & & & 1533.8 & $1.5 \mathrm{E}+04$ & \multicolumn{2}{|c|}{ no labelling } & \\
\hline & & & & 1570.9 & $3.5 \mathrm{E}+04$ & \multicolumn{2}{|c|}{ no labelling } & \\
\hline & & & & 2202.9 & $9.3 \mathrm{E}+04$ & \multicolumn{2}{|c|}{ no labelling } & \\
\hline \multicolumn{9}{|c|}{ intracellulare } \\
\hline \multirow[t]{9}{*}{ O75417 } & $\begin{array}{l}\text { DNA } \\
\text { polymerase } \\
\text { theta }\end{array}$ & 66 & 99.704 & 980.5 & $1.8 \mathrm{E}+04$ & no & abeling & \\
\hline & & & & 1115.5 & $3.7 \mathrm{E}+03$ & \multicolumn{2}{|c|}{ no labeling } & \\
\hline & & & & 1121.5 & 8.7E+03 & \multicolumn{2}{|c|}{ no labeling } & \\
\hline & & & & 1165.7 & $1.3 E+04$ & \multicolumn{2}{|c|}{ no labeling } & \\
\hline & & & & 1323.6 & $3.1 \mathrm{E}+04$ & \multicolumn{2}{|c|}{ no labeling } & \\
\hline & & & & 1516.7 & $2.6 \mathrm{E}+04$ & 1521.7 & $7.8 \mathrm{E}+04$ & $29.89 \%$ \\
\hline & & & & 1547.7 & $3.5 \mathrm{E}+03$ & \multicolumn{2}{|c|}{ no labeling } & \\
\hline & & & & 1750.7 & $6.2 E+03$ & \multicolumn{2}{|c|}{ no labeling } & \\
\hline & & & & 1751.7 & $3.0 \mathrm{E}+03$ & \multicolumn{2}{|c|}{ no labeling } & \\
\hline \multirow[t]{2}{*}{ O75417 } & $\begin{array}{l}\text { DNA } \\
\text { polymerase } \\
\text { theta }\end{array}$ & 66 & 99.704 & 1790.8 & $1.6 \mathrm{E}+04$ & \multicolumn{2}{|c|}{ no labeling } & \\
\hline & & & & 1949.9 & $5.5 \mathrm{E}+03$ & 1955.0 & $5.9 E+04$ & $108.64 \%$ \\
\hline \multirow[t]{2}{*}{ Q9NUR3 } & $\begin{array}{l}\text { Protein } \\
\text { C20orf46 }\end{array}$ & 58 & 97.955 & 948.5 & $3.4 \mathrm{E}+03$ & \multicolumn{2}{|c|}{ no labeling } & \\
\hline & & & & 1559.7 & $3.4 \mathrm{E}+03$ & 1564.7 & 3256.32 & $96.20 \%$ \\
\hline O15482 & $\begin{array}{l}\text { Testis-specific } \\
\text { protein TEX28 }\end{array}$ & 55 & 96.013 & & & no $F$ & & \\
\hline \multicolumn{9}{|c|}{ Adipocytes controle } \\
\hline \multicolumn{9}{|c|}{ classical secreted } \\
\hline \multirow[t]{4}{*}{ P08253 } & $\begin{array}{l}72 \text { kDa type IV } \\
\text { collagenase } \\
\text { precursor }\end{array}$ & 77 & 99.975 & 1407.5 & $5.5 \mathrm{E}+03$ & 1412.6 & $3.7 E+03$ & $67.60 \%$ \\
\hline & & & & 1418.7 & $2.6 \mathrm{E}+04$ & 1423.7 & $1.7 E+04$ & $65.06 \%$ \\
\hline & & & & 1603.7 & $8.8 \mathrm{E}+03$ & 1608.7 & $5.8 E+03$ & $65.66 \%$ \\
\hline & & & & 2108.0 & $3.7 \mathrm{E}+03$ & 2113.0 & $4.2 E+03$ & $112.82 \%$ \\
\hline \multirow[t]{2}{*}{ P02649 } & $\begin{array}{l}\text { Apolipoprotein } \\
\text { E precursor }\end{array}$ & 60 & 98.68 & 1033.5 & $1.1 \mathrm{E}+04$ & 1038.5 & $9.0 \mathrm{E}+03$ & $82.19 \%$ \\
\hline & & & & 1552.6 & $1.1 \mathrm{E}+04$ & 1557.7 & $8.5 E+03$ & $75.35 \%$ \\
\hline P02650 & $\begin{array}{l}\text { Apolipoprotein } \\
\text { E precursor }\end{array}$ & 131 & 100 & 1033.5 & $1.2 \mathrm{E}+04$ & 1038.5 & $6.1 \mathrm{E}+03$ & $52.13 \%$ \\
\hline & & & & 1552.6 & $8.9 E+03$ & 1557.7 & $5.4 \mathrm{E}+03$ & $60.86 \%$ \\
\hline P02651 & $\begin{array}{l}\text { Apolipoprotein } \\
\text { E precursor }\end{array}$ & 81 & 99.99 & 1536.6 & $4.4 \mathrm{E}+03$ & 1541.7 & $4.1 \mathrm{E}+03$ & $92.40 \%$ \\
\hline & & & & 1552.7 & $1.3 \mathrm{E}+04$ & 1557.7 & $1.1 \mathrm{E}+04$ & $82.83 \%$ \\
\hline
\end{tabular}


Table of Supplement 3: Overview of the identified proteins derived from the protein turnover experiments

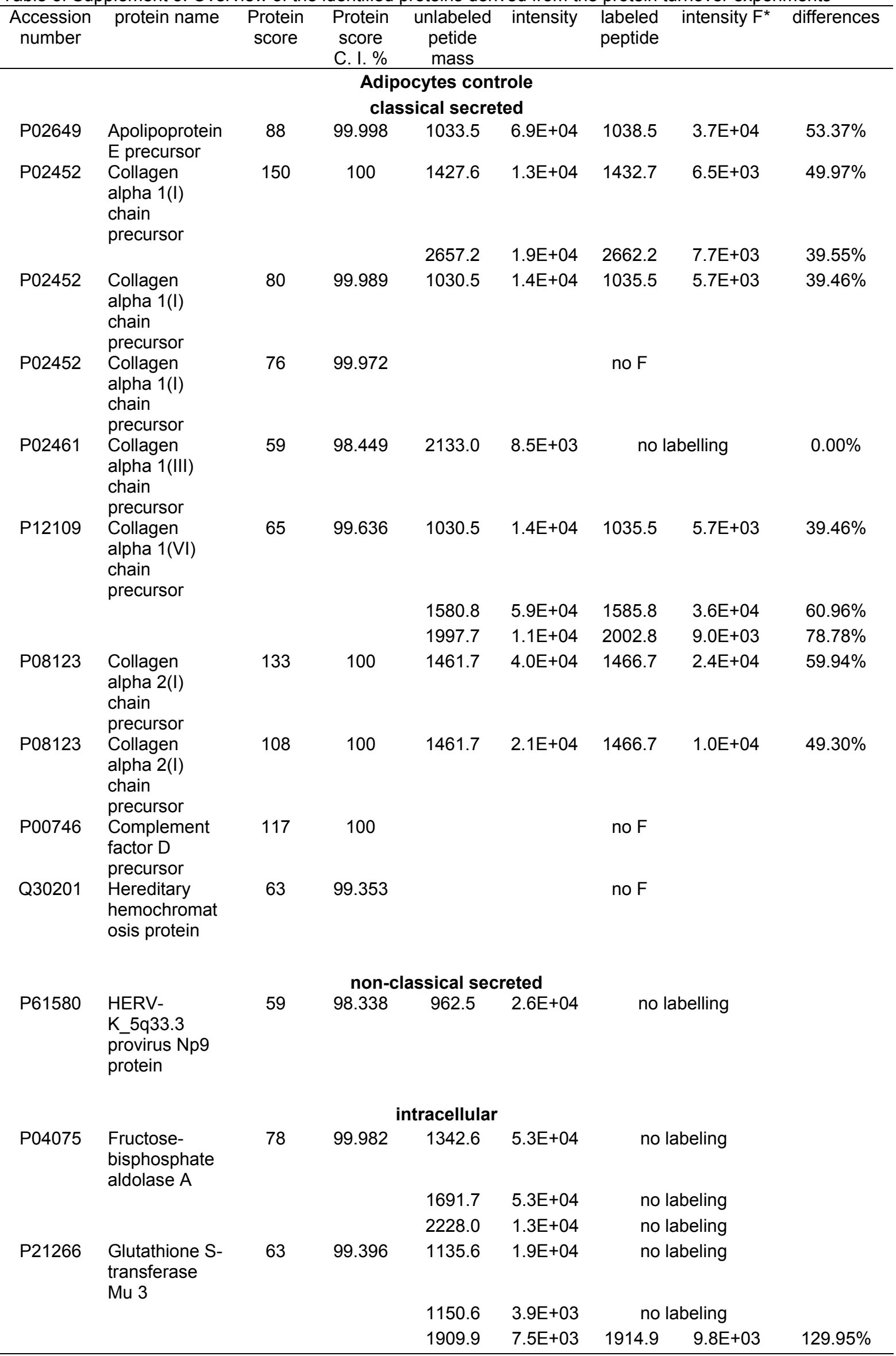


Table of Supplement 3: Overview of the identified proteins derived from the protein turnover experiments

\begin{tabular}{|c|c|c|c|c|c|c|c|c|}
\hline $\begin{array}{l}\text { Accession } \\
\text { number }\end{array}$ & protein name & $\begin{array}{l}\text { Protein } \\
\text { score }\end{array}$ & $\begin{array}{l}\text { Protein } \\
\text { score } \\
\text { C. I. \% }\end{array}$ & $\begin{array}{l}\text { unlabeled } \\
\text { petide } \\
\text { mass }\end{array}$ & intensity & $\begin{array}{l}\text { labeled } \\
\text { peptide }\end{array}$ & intensity $\mathrm{F}^{*}$ & differences \\
\hline \multicolumn{9}{|c|}{ Adipocytes controle } \\
\hline \multicolumn{9}{|c|}{ intracellular } \\
\hline \multirow[t]{6}{*}{ P30041 } & $\begin{array}{l}\text { Peroxiredoxin } \\
6\end{array}$ & 63 & 99.424 & 906.4 & $1.8 \mathrm{E}+04$ & \multicolumn{3}{|c|}{ no labeling } \\
\hline & & & & 1085.5 & $6.3 E+04$ & \multicolumn{2}{|c|}{ no labeling } & \\
\hline & & & & 1135.6 & $1.9 \mathrm{E}+04$ & \multicolumn{2}{|c|}{ no labeling } & \\
\hline & & & & 1395.6 & $8.9 E+03$ & \multicolumn{2}{|c|}{ no labeling } & \\
\hline & & & & 1665.7 & $3.8 \mathrm{E}+03$ & \multicolumn{2}{|c|}{ no labeling } & \\
\hline & & & & 2098.0 & $4.2 \mathrm{E}+03$ & \multicolumn{2}{|c|}{ no labeling } & \\
\hline \multirow[t]{3}{*}{ Q9Y5X3 } & $\begin{array}{l}\text { Sorting nexin } \\
5\end{array}$ & 55 & 95.92 & 1165.7 & $2.4 \mathrm{E}+04$ & \multicolumn{2}{|c|}{ no labeling } & \\
\hline & & & & 1302.5 & $5.6 \mathrm{E}+03$ & \multicolumn{2}{|c|}{ no labeling } & \\
\hline & & & & 1578.7 & $1.7 E+05$ & \multicolumn{2}{|c|}{ no labeling } & \\
\hline \multirow[t]{3}{*}{095347} & $\begin{array}{l}\text { Structural } \\
\text { maintenance } \\
\text { of } \\
\text { chromosome } \\
\text { 2-like }\end{array}$ & 56 & 97.177 & 1383.7 & $1.3 \mathrm{E}+04$ & 1388.7 & $6.1 \mathrm{E}+03$ & $47.21 \%$ \\
\hline & & & & 1511.7 & $2.2 \mathrm{E}+04$ & \multicolumn{2}{|c|}{ no labeling } & \\
\hline & & & & 1563.7 & $7.4 \mathrm{E}+03$ & \multicolumn{2}{|c|}{ no labeling } & \\
\hline \multirow[t]{5}{*}{ P60174 } & $\begin{array}{l}\text { Triosephosph } \\
\text { ate isomerase }\end{array}$ & 62 & 99.323 & 954.4 & $5.2 \mathrm{E}+03$ & \multicolumn{2}{|c|}{ no labeling } & \\
\hline & & & & 1082.5 & $2.0 \mathrm{E}+03$ & \multicolumn{2}{|c|}{ no labeling } & \\
\hline & & & & 1458.6 & $1.4 \mathrm{E}+04$ & \multicolumn{2}{|c|}{ no labeling } & \\
\hline & & & & 1614.7 & $1.2 \mathrm{E}+04$ & \multicolumn{2}{|c|}{ no labeling } & \\
\hline & & & & 2191.9 & $1.2 \mathrm{E}+04$ & \multicolumn{2}{|c|}{ no labeling } & \\
\hline \multirow[t]{7}{*}{ Q8N7K0 } & $\begin{array}{l}\text { Zinc finger } \\
\text { protein } 433\end{array}$ & 55 & 96.103 & 1177.5 & $2.1 \mathrm{E}+03$ & \multicolumn{2}{|c|}{ no labeling } & \\
\hline & & & & 1448.8 & $3.1 \mathrm{E}+03$ & \multicolumn{2}{|c|}{ no labeling } & \\
\hline & & & & 1522.7 & $2.8 \mathrm{E}+04$ & \multicolumn{2}{|c|}{ no labeling } & \\
\hline & & & & 1708.8 & $3.8 \mathrm{E}+03$ & \multicolumn{2}{|c|}{ no labeling } & \\
\hline & & & & 2211.0 & $4.0 \mathrm{E}+04$ & no I & abeling & \\
\hline & & & Prea & ipocytes C & $\mathrm{Cl} 2$ & & & \\
\hline & & & clas & sical secre & & & & \\
\hline P02452 & $\begin{array}{l}\text { Collagen alpha } \\
1(\mathrm{I}) \text { chain } \\
\text { precursor }\end{array}$ & 182 & 100 & 1427.7 & $1.8 \mathrm{E}+04$ & 1432.7 & $5.2 \mathrm{E}+03$ & $29.70 \%$ \\
\hline & & & & 1537.8 & $1.1 \mathrm{E}+04$ & 1542.8 & $4.3 E+03$ & $39.05 \%$ \\
\hline P02452 & $\begin{array}{l}\text { Collagen alpha } \\
1(\mathrm{I}) \text { chain } \\
\text { precursor }\end{array}$ & 51 & 90.212 & & & no $F$ & & \\
\hline P12109 & $\begin{array}{l}\text { Collagen alpha } \\
1(\mathrm{VI}) \text { chain } \\
\text { precursor }\end{array}$ & 64 & 99.563 & 1580.8 & $1.3 \mathrm{E}+04$ & 1585.8 & $3.6 \mathrm{E}+03$ & $27.60 \%$ \\
\hline P09486 & $\begin{array}{l}\text { SPARC } \\
\text { precursor }\end{array}$ & 58 & 98.135 & 1950.0 & $8.8 \mathrm{E}+03$ & 1955.0 & $2.2 \mathrm{E}+03$ & $24.81 \%$ \\
\hline Q7Z7G0 & $\begin{array}{l}\text { Target of } \\
\text { Nesh-SH3 } \\
\text { precursor }\end{array}$ & 69 & 99.855 & 1975.9 & $6.8 \mathrm{E}+03$ & 1980.9 & $4.8 E+03$ & $70.39 \%$ \\
\hline & & & & & & 1985.9 & $2.5 \mathrm{E}+03$ & $37.45 \%$ \\
\hline & & & & 2205.0 & $5.0 \mathrm{E}+03$ & 2210.0 & $1.7 E+03$ & $34.02 \%$ \\
\hline
\end{tabular}


Table of Supplement 3: Overview of the identified proteins derived from the protein turnover experiments

\begin{tabular}{|c|c|c|c|c|c|c|c|c|}
\hline $\begin{array}{l}\text { Accession } \\
\text { number }\end{array}$ & protein name & $\begin{array}{l}\text { Protein } \\
\text { score }\end{array}$ & $\begin{array}{l}\text { Protein } \\
\text { score } \\
\text { C. I. } \%\end{array}$ & $\begin{array}{l}\text { unlabeled } \\
\text { petide } \\
\text { mass }\end{array}$ & intensity & $\begin{array}{l}\text { labeled } \\
\text { peptide }\end{array}$ & intensity $\mathrm{F}^{*}$ & differences \\
\hline \multicolumn{9}{|c|}{ Preadipocytes $\mathrm{CoCl} 2$} \\
\hline \multicolumn{9}{|c|}{ non-classical secreted } \\
\hline \multirow[t]{5}{*}{ P30044 } & $\begin{array}{l}\text { Peroxiredoxin } \\
5, \\
\text { mitochondrial } \\
\text { precursor }\end{array}$ & 61 & 98.998 & 933.5 & $5.4 \mathrm{E}+03$ & \multicolumn{3}{|c|}{ no labelling } \\
\hline & & & & 1089.6 & $5.9 \mathrm{E}+03$ & \multicolumn{3}{|c|}{ no labelling } \\
\hline & & & & 1344.7 & $3.4 \mathrm{E}+04$ & \multicolumn{3}{|c|}{ no labelling } \\
\hline & & & & 1539.7 & $1.4 \mathrm{E}+04$ & \multicolumn{3}{|c|}{ no labelling } \\
\hline & & & & 1827.9 & $2.3 \mathrm{E}+03$ & \multicolumn{3}{|c|}{ no labelling } \\
\hline \multicolumn{9}{|c|}{ intracellular } \\
\hline Q9C0H9 & $\begin{array}{l}\text { p130Cas- } \\
\text { associated } \\
\text { protein }\end{array}$ & 60 & 98.796 & 1046.6 & $2.6 \mathrm{E}+03$ & \multicolumn{3}{|c|}{ no labeling } \\
\hline P07311 & $\begin{array}{l}\text { Acylphosphata } \\
\text { se, organ- } \\
\text { common type } \\
\text { isozyme }\end{array}$ & 55 & 95.825 & 980.6 & $9.8 \mathrm{E}+02$ & \multicolumn{3}{|c|}{ no labeling } \\
\hline \multirow[t]{3}{*}{ P22392 } & $\begin{array}{l}\text { Nucleoside } \\
\text { diphosphate } \\
\text { kinase B }\end{array}$ & 56 & 96.975 & 1051.5 & $4.0 \mathrm{E}+03$ & \multicolumn{3}{|c|}{ no labeling } \\
\hline & & & & 1175.6 & $2.2 \mathrm{E}+04$ & \multirow{2}{*}{\multicolumn{2}{|c|}{$\begin{array}{l}\text { no labeling } \\
\text { no labeling }\end{array}$}} & \\
\hline & & & & 1344.7 & $3.4 \mathrm{E}+04$ & & & \\
\hline \multicolumn{9}{|c|}{ Preadipocyte controlle } \\
\hline \multicolumn{9}{|c|}{ classical secreted } \\
\hline \multirow[t]{5}{*}{ P08253 } & $\begin{array}{l}72 \text { kDa type IV } \\
\text { collagenase } \\
\text { precursor }\end{array}$ & 57 & 97.705 & 1407.5 & $7.0 \mathrm{E}+03$ & 1412.6 & $3.5 \mathrm{E}+03$ & $50.51 \%$ \\
\hline & & & & 1418.7 & $3.0 \mathrm{E}+04$ & 1423.7 & $9.0 \mathrm{E}+03$ & $30.49 \%$ \\
\hline & & & & 1603.705 & $9.3 \mathrm{E}+03$ & 1608.7 & $5.6 \mathrm{E}+03$ & $60.36 \%$ \\
\hline & & & & 2107.931 & $1.0 \mathrm{E}+04$ & 2113.0 & 7.7E+03 & $74.35 \%$ \\
\hline & & & & 2198.964 & $5.6 \mathrm{E}+03$ & 2204.0 & $3.7 \mathrm{E}+03$ & $65.40 \%$ \\
\hline \multirow[t]{2}{*}{ P02452 } & $\begin{array}{l}\text { Collagen alpha } \\
1(\mathrm{I}) \text { chain } \\
\text { precursor }\end{array}$ & 56 & 96.606 & & & no $F$ & & \\
\hline & & & & 1828.8 & $6.9 \mathrm{E}+03$ & 1833.8 & $5.1 \mathrm{E}+03$ & $73.61 \%$ \\
\hline P08123 & $\begin{array}{l}\text { Collagen alpha } \\
2(I) \text { chain } \\
\text { precursor }\end{array}$ & 58 & 97.955 & 1461.7 & $2.6 \mathrm{E}+04$ & 1466.7 & $6.3 \mathrm{E}+03$ & $24.26 \%$ \\
\hline \multirow[t]{5}{*}{ P09382 } & Galectin-1 & 73 & 99.934 & 968.4 & $8.0 E+03$ & \multicolumn{3}{|c|}{ no labelling } \\
\hline & & & & 1482.7 & 7.7E+03 & \multicolumn{3}{|c|}{ no labelling } \\
\hline & & & & 1486.7 & $9.4 \mathrm{E}+04$ & \multicolumn{3}{|c|}{ no labelling } \\
\hline & & & & 1800.9 & $1.2 E+04$ & \multicolumn{3}{|c|}{ no labelling } \\
\hline & & & & 2299.2 & $1.1 \mathrm{E}+05$ & \multicolumn{2}{|c|}{ no labelling } & \\
\hline \multirow[t]{4}{*}{ P09382 } & Galectin-1 & 62 & 99.223 & 1486.6 & $4.4 \mathrm{E}+04$ & \multicolumn{2}{|c|}{ no labelling } & \\
\hline & & & & 1647.7 & $2.2 \mathrm{E}+03$ & \multicolumn{2}{|c|}{ no labelling } & \\
\hline & & & & 1800.8 & $2.0 \mathrm{E}+03$ & no l & abelling & \\
\hline & & & & 2299.1 & $2.3 E+04$ & no l & abelling & \\
\hline P01033 & $\begin{array}{l}\text { Metalloproteina } \\
\text { se inhibitor } 1 \\
\text { precursor }\end{array}$ & 54 & 95.094 & 1233.6 & $2.3 \mathrm{E}+03$ & 1238.6 & $6.9 \mathrm{E}+02$ & $30.39 \%$ \\
\hline P09486 & $\begin{array}{l}\text { SPARC } \\
\text { precursor }\end{array}$ & 59 & 98.617 & 1949.9 & $1.2 \mathrm{E}+05$ & 1955.0 & $4.0 \mathrm{E}+04$ & $32.78 \%$ \\
\hline
\end{tabular}


Table of Supplement 3: Overview of the identified proteins derived from the protein turnover experiments

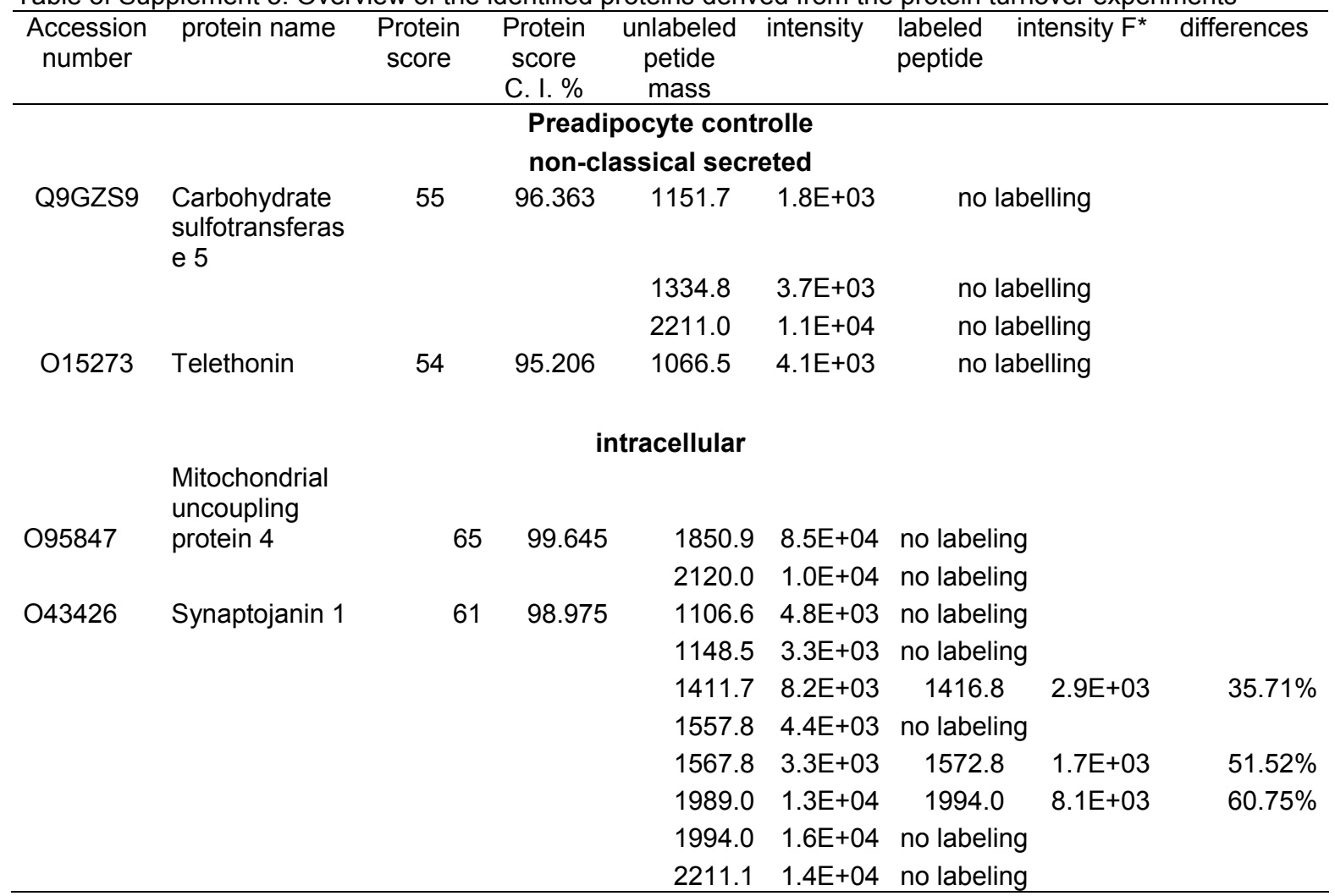

Table of Supplement 3: Overview of the identified proteins derived from the protein turnover experiments, including labeling levels. All four conditions including (pre)adipocytes incubated with or without $\mathrm{CoCl}_{2}$ are shown separately for ideal comparison. MS data related to the identifications are deposited on the PRIDE server (project accession number: 28672-28710). Proteins marked with * are identified as novel (pre)adipocytes secreted proteins.

\section{Supplemental data chapter 6}

Supplement 1 of 2 is shown at the websit of Journal of Proteomic Research (http://pubs.acs.org/doi/suppl/10.1021/pr300539b).

\section{Supplemental data chapter 7}

\section{Suplement 1}

Supplemental Table 1 is a summary of MS/MS-related data of all identified proteins of calorie restricted SGBS adipocytes including spot number, protein name, peptide information and molecular weight. Further detailed information is deposited on the PRIDE server (project accession number: 29652-29779). In addition, statistical calculations (student T-test) are shown for all differentially expressed spots from the 2D gels.

This Excel table is shown at https://www.dropbox.com/sh/0mas5n7hfdscaxo/WVB5_52Tso as Supplemental data Chapter 7_Supplement 1. 
Table of Supplement 1: Identifications of secreted proteins of calorie restricted and non-calorie restricted SGBS adipocytes

\begin{tabular}{|c|c|c|c|c|c|c|}
\hline \multirow[t]{3}{*}{$\begin{array}{c}\text { accession } \\
\text { number }\end{array}$} & \multicolumn{2}{|l|}{ Protein name } & \multirow[t]{2}{*}{ ID } & \multirow[t]{2}{*}{$\begin{array}{c}\mathrm{MW} \\
{[\mathrm{kDa}]}\end{array}$} & \multirow[t]{2}{*}{$\begin{array}{c}\text { fold } \\
\text { change }\end{array}$} & \multirow[t]{2}{*}{$p$-value } \\
\hline & classical & secreted & & & & \\
\hline & extracellul & ar matrix & & & & \\
\hline \multirow[t]{13}{*}{ P02452 } & Collagen alpha- $1(\mathrm{I})$ chain & (C-term) & 15 & 34,7 & $-5.40 E+04$ & 4.45E-02 \\
\hline & & & 15 & 217,5 & $-3.40 \mathrm{E}+00$ & $9.55 \mathrm{E}-02$ \\
\hline & & & 15 & 248,3 & $7.40 E+03$ & 2.29E-03 \\
\hline & & & 15 & 248,8 & $3.10 E+04$ & 2.27E-05 \\
\hline & & (C-term) & 18 & 34,2 & $-2.00 E+00$ & 5.17E-02 \\
\hline & & (C-term) & 18 & 31,9 & $8.20 E+04$ & 1.04E-02 \\
\hline & & (C-term) & 18 & 35,0 & $-2.80 E+00$ & 7.07E-02 \\
\hline & & & 37 & 219,3 & $-2.40 E+00$ & $9.32 \mathrm{E}-02$ \\
\hline & & & 37 & 221,0 & $-3.90 E+00$ & 4.27E-02 \\
\hline & & & 37 & 251,4 & $7.90 \mathrm{E}+00$ & 2.87E-02 \\
\hline & & (C-term) & 46 & 47,2 & $4.60 E+00$ & $9.70 \mathrm{E}-02$ \\
\hline & & & 66 & 243,2 & $5.20 \mathrm{E}+00$ & $9.22 \mathrm{E}-02$ \\
\hline & & & 80 & 249,8 & $1.10 \mathrm{E}+01$ & 4.70E-03 \\
\hline \multirow[t]{8}{*}{ P02461 } & Collagen alpha-1(III) chain & (C-term) & 15 & 34,7 & $-5.40 E+04$ & 4.45E-02 \\
\hline & & & 15 & 217,5 & $-3.40 E+00$ & 9.55E-02 \\
\hline & & & 15 & 248,3 & $7.40 \mathrm{E}+03$ & 2.29E-03 \\
\hline & & & 15 & 248,8 & $3.10 E+04$ & 2.27E-05 \\
\hline & & (C-term) & 21 & 50,0 & $-4.00 E+00$ & $6.02 \mathrm{E}-02$ \\
\hline & & (C-term) & 30 & 33,4 & $-2.90 \mathrm{E}+00$ & $1.83 \mathrm{E}-02$ \\
\hline & & (C-term) & 31 & 30,8 & $2.40 \mathrm{E}+00$ & 7.85E-08 \\
\hline & & & 66 & 243,2 & $5.20 \mathrm{E}+00$ & $9.22 \mathrm{E}-02$ \\
\hline P02462 & Collagen alpha-1(IV) chain (Isoform 1) & & 67 & 254,7 & $3.20 \mathrm{E}+00$ & 4.10E-02 \\
\hline \multirow[t]{5}{*}{ P20908 } & Collagen alpha-1(V) chain & (C-term) & 1 & 35,3 & $-5.00 E+00$ & 5.13E-02 \\
\hline & & (C-term) & 2 & 36,0 & $-4.40 E+00$ & 7.60E-02 \\
\hline & & & 13 & 125,0 & $-2.00 E+00$ & $1.20 \mathrm{E}-03$ \\
\hline & & (N-term) & 25 & 74,0 & $-4.90 E+04$ & 4.98E-02 \\
\hline & & & 28 & 196,7 & $-1.30 E+00$ & 2.25E-02 \\
\hline \multirow[t]{13}{*}{ P08123 } & Collagen alpha-2(I) chain & (C-term) & 40 & 28,7 & $6.10 E+04$ & 1.30E-06 \\
\hline & & & 40 & 48,4 & $2.10 E+00$ & $9.20 \mathrm{E}-02$ \\
\hline & & & 40 & 206,3 & $7.60 \mathrm{E}+00$ & 1.95E-02 \\
\hline & & & 40 & 203,2 & $5.70 \mathrm{E}+00$ & $5.05 E-02$ \\
\hline & & & 40 & 205,2 & $2.50 E+01$ & 4.55E-02 \\
\hline & & & 40 & 199,7 & $1.30 \mathrm{E}+01$ & 4.82E-02 \\
\hline & & (C-term) & 44 & 31,0 & $-3.20 E+00$ & 1.05E-02 \\
\hline & & (C-term) & 45 & 28,6 & $1.40 \mathrm{E}+04$ & 2.56E-02 \\
\hline & & & 67 & 254,7 & $3.20 \mathrm{E}+00$ & 4.10E-02 \\
\hline & & & 80 & 249,8 & $1.10 E+01$ & 4.70E-03 \\
\hline & & & 81 & 248,5 & $5.80 \mathrm{E}+00$ & $9.08 \mathrm{E}-02$ \\
\hline & & & 88 & 200,0 & $9.60 \mathrm{E}+02$ & 3.49E-02 \\
\hline & & & 89 & 200,6 & $1.60 \mathrm{E}+01$ & $7.42 \mathrm{E}-02$ \\
\hline \multirow[t]{4}{*}{ P08572 } & Collagen alpha-2(IV) chain & & 67 & 254,7 & $3.20 \mathrm{E}+00$ & 4.10E-02 \\
\hline & & & 80 & 249,8 & $1.10 \mathrm{E}+01$ & 4.70E-03 \\
\hline & & & 81 & 248,5 & $5.80 \mathrm{E}+00$ & $9.08 \mathrm{E}-02$ \\
\hline & & & 87 & 247,8 & $-5.70 E+00$ & 2.49E-03 \\
\hline P12110 & Collagen alpha-2(VI) chain (Isoform $2 \mathrm{C} 2 \mathrm{~A}$ & & 53 & 201,9 & $-4.80 E+00$ & 2.23E-02 \\
\hline P02751 & Fibronectin isoform 1 & & 27 & 308,5 & $-8.70 E+00$ & 2.67E-03 \\
\hline D3DQH8 & SPARC & & 11 & 44,5 & $-4.40 E+00$ & 1.88E-02 \\
\hline
\end{tabular}


Table of Supplement 1: Identifications of secreted proteins of calorie restricted and non-calorie restricted SGBS adipocytes

\begin{tabular}{|c|c|c|c|c|c|}
\hline $\begin{array}{l}\text { accession } \\
\text { number }\end{array}$ & Protein name & ID & $\begin{array}{c}\mathrm{MW} \\
{[\mathrm{kDa}]}\end{array}$ & $\begin{array}{c}\text { fold } \\
\text { change }\end{array}$ & p-value \\
\hline \multicolumn{6}{|c|}{$\begin{array}{c}\text { classical secreted } \\
\text { processing }\end{array}$} \\
\hline P07711 & Cathepsin L1 & 34 & 39,7 & $5.30 \mathrm{E}+04$ & 6.62E-02 \\
\hline Q9UBR2 & Cathepsin Z & 60 & 35,8 & $2.30 \mathrm{E}+00$ & 5.88E-02 \\
\hline \multirow[t]{2}{*}{ P01033 } & \multirow[t]{2}{*}{ Metalloproteinase inhibitor 1} & 74 & 29,5 & $1.90 \mathrm{E}+00$ & 5.05E-02 \\
\hline & & 74 & 30,3 & $2.20 \mathrm{E}+04$ & 8.49E-03 \\
\hline \multirow[t]{2}{*}{ Q15113 } & Procollagen C-endopeptidase enhancer 1 & 63 & 47,6 & $3.90 \mathrm{E}+04$ & 2.87E-02 \\
\hline & \multicolumn{2}{|l|}{ regulation/signaling } & & & \\
\hline B4DV10 & $\begin{array}{l}\text { cDNA FLJ59142. highly similar to Epididymal secretory } \\
\text { protein E1 * }\end{array}$ & 70 & 13,1 & $8.90 \mathrm{E}+00$ & 9.81E-02 \\
\hline \multirow[t]{3}{*}{ Q15848 } & \multirow[t]{3}{*}{ Adiponectin } & 19 & 36,3 & $-3.30 \mathrm{E}+00$ & 3.89E-02 \\
\hline & & 19 & 31,0 & $-5.90 \mathrm{E}+00$ & $6.42 \mathrm{E}-02$ \\
\hline & & 31 & 30,8 & $2.40 \mathrm{E}+00$ & 7.85E-08 \\
\hline \multirow[t]{2}{*}{095841} & \multirow[t]{2}{*}{ Angiopoietin-related protein 1} & 66 & 243,2 & $5.20 \mathrm{E}+00$ & $9.22 \mathrm{E}-02$ \\
\hline & & 77 & 40,6 & $2.50 \mathrm{E}+00$ & $6.01 \mathrm{E}-02$ \\
\hline P01019 & Angiotensinogen & 25 & 74,0 & $-4.90 E+04$ & 4.98E-02 \\
\hline \multirow{8}{*}{ P02649 } & \multirow{8}{*}{ Apolipoprotein E } & 1 & 35,3 & $-5.00 \mathrm{E}+00$ & 5.13E-02 \\
\hline & & 8 & 37,8 & $4.90 \mathrm{E}+03$ & 2.62E-02 \\
\hline & & 8 & 24,6 & $-4.10 E+00$ & 7.00E-07 \\
\hline & & 18 & 34,2 & $-2.00 \mathrm{E}+00$ & 5.17E-02 \\
\hline & & 18 & 31,9 & $8.20 E+04$ & 1.04E-02 \\
\hline & & 18 & 35,0 & $-2.80 \mathrm{E}+00$ & 7.07E-02 \\
\hline & & 19 & 36,3 & $-3.30 E+00$ & 3.89E-02 \\
\hline & & 19 & 31,0 & $-5.90 \mathrm{E}+00$ & $6.42 \mathrm{E}-02$ \\
\hline D6QS48 & Calumenin (isoform 4) & 6 & 32,1 & $-3.80 E+00$ & 2.10E-02 \\
\hline Q15782 & Chitinase-3-like protein 2 & 77 & 40,6 & $2.50 \mathrm{E}+00$ & $6.01 \mathrm{E}-02$ \\
\hline Q96AJ1 & Clusterin (Isoform 1) & 32 & 36.3 & $-6.60 E+00$ & 1.31E-02 \\
\hline P14625 & Endoplasmin & 12 & 58,1 & $6.70 \mathrm{E}+03$ & $1.56 \mathrm{E}-02$ \\
\hline Q12841 & Follistatin-related protein 1 & 9 & 46,1 & $-3.30 E+00$ & 2.32E-02 \\
\hline \multirow[t]{2}{*}{ P06396 } & \multirow[t]{2}{*}{ Gelsolin (Isoform 1) } & 52 & 116,7 & $-2.00 \mathrm{E}+00$ & 7.80E-02 \\
\hline & & 52 & 117,1 & $-6.40 \mathrm{E}+00$ & $9.41 \mathrm{E}-02$ \\
\hline P00738 & Haptoglobin & 38 & 16,7 & $-8.80 \mathrm{E}+00$ & $6.96 \mathrm{E}-02$ \\
\hline \multirow[t]{11}{*}{ P36955 } & \multirow[t]{11}{*}{ Pigment epithelium-derived factor } & 21 & 50,0 & $-4.00 E+00$ & $6.02 E-02$ \\
\hline & & 22 & 49,3 & $-2.70 \mathrm{E}+00$ & 7.38E-02 \\
\hline & & 23 & 51,3 & $-9.80 E+00$ & $1.62 \mathrm{E}-02$ \\
\hline & & 23 & 49,3 & $-2.50 \mathrm{E}+00$ & 6.77E-02 \\
\hline & & 23 & 49,5 & $-4.50 E+00$ & 7.94E-03 \\
\hline & & 23 & 48,5 & $-4.00 E+00$ & 1.04E-02 \\
\hline & & 23 & 46,3 & $1.10 \mathrm{E}+01$ & 3.82E-02 \\
\hline & & 46 & 47,2 & $4.60 \mathrm{E}+00$ & $9.70 \mathrm{E}-02$ \\
\hline & & 48 & 42,2 & $6.20 \mathrm{E}+03$ & 1.97E-03 \\
\hline & & 49 & 47,7 & $-2.00 \mathrm{E}+00$ & 8.42E-02 \\
\hline & & 66 & 243,2 & $5.20 \mathrm{E}+00$ & $9.22 \mathrm{E}-02$ \\
\hline \multirow[t]{2}{*}{ P05121 } & \multirow[t]{2}{*}{ Plasminogen activator inhibitor 1} & 63 & 47,6 & $3.90 \mathrm{E}+04$ & 2.87E-02 \\
\hline & & 76 & 42,8 & $7.10 \mathrm{E}+03$ & 3.47E-02 \\
\hline \multirow[t]{3}{*}{ P41222 } & \multirow[t]{3}{*}{ Prostaglandin-H2 D-isomerase } & 16 & 27,1 & $-1.50 \mathrm{E}+01$ & 2.15E-02 \\
\hline & & 42 & 27,0 & $1.40 \mathrm{E}+05$ & 8.17E-02 \\
\hline & & 16 & 16,4 & $-4.40 E+00$ & 1.91E-03 \\
\hline P10599 & Thioredoxin & 3 & 9,3 & $3.40 \mathrm{E}+04$ & 7.21E-04 \\
\hline Q8NBS9 & Thioredoxin domain-containing protein 5 & 22 & 49,3 & $-2.70 \mathrm{E}+00$ & 7.38E-02 \\
\hline
\end{tabular}


Table of Supplement 1: Identifications of secreted proteins of calorie restricted and non-calorie restricted SGBS adipocytes

\begin{tabular}{|c|c|c|c|c|c|}
\hline $\begin{array}{l}\text { accession } \\
\text { number }\end{array}$ & Protein name & ID & $\begin{array}{c}\mathrm{MW} \\
{[\mathrm{kDa}]}\end{array}$ & $\begin{array}{c}\text { fold } \\
\text { change }\end{array}$ & p-value \\
\hline & $\begin{array}{l}\text { classical secreted } \\
\text { regulation/signaling }\end{array}$ & & & & \\
\hline \multirow[t]{6}{*}{ P25311 } & Zinc-alpha-2-glycoprotein & 7 & 43,2 & $-9.30 E+00$ & 3.02E-02 \\
\hline & & 7 & 43,9 & $-8.40 E+00$ & 3.92E-02 \\
\hline & & 7 & 42,2 & $-9.00 E+04$ & 9.62E-03 \\
\hline & & 7 & 31,7 & $9.20 \mathrm{E}+04$ & 1.83E-02 \\
\hline & & 11 & 44,5 & $-4.40 E+00$ & 1.88E-02 \\
\hline & immune regulation & & & & \\
\hline \multirow[t]{3}{*}{ B4DPQ0 } & $\begin{array}{l}\text { cDNA FLJ54471. highly similar to Complement C1r } \\
\text { subcomponent * }\end{array}$ & 29 & 123,0 & $-4.00 E+00$ & 3.97E-02 \\
\hline & & 29 & 121,7 & $-4.50 E+00$ & 4.59E-02 \\
\hline & & 29 & 123,4 & $-2.50 E+00$ & 3.33E-02 \\
\hline \multirow[t]{4}{*}{ P09871 } & Complement $\mathrm{C} 1 \mathrm{~s}$ subcomponent & 13 & 125,0 & $-2.00 E+00$ & $1.20 \mathrm{E}-03$ \\
\hline & & 14 & 122,2 & $-3.40 E+00$ & 4.91E-02 \\
\hline & & 14 & 119,8 & $3.20 \mathrm{E}+00$ & 7.69E-02 \\
\hline & & 14 & 111,3 & $1.50 \mathrm{E}+04$ & 7.17E-06 \\
\hline \multirow[t]{2}{*}{ P06702 } & Protein S100-A9 & 51 & 249,6 & $8.10 \mathrm{E}+00$ & 2.43E-03 \\
\hline & non-classical secreted & & & & \\
\hline Q6NVY1 & $\begin{array}{l}\text { 3-hydroxyisobutyryl-CoA hydrolase. mitochondrial } \\
\text { (Isoform 1) * }\end{array}$ & 75 & 40,1 & $-5.80 E+00$ & 4.59E-02 \\
\hline P08865 & $40 S$ ribosomal protein SA & 11 & 44,5 & $-4.40 E+00$ & 1.88E-02 \\
\hline B4DWA6 & $\begin{array}{l}\text { cDNA FLJ60094. highly similar to F-actin capping } \\
\text { protein subunit beta * }\end{array}$ & 44 & 31,0 & $-3.20 E+00$ & 1.05E-02 \\
\hline \multirow[t]{2}{*}{ P06733 } & Alpha-enolase (Isoform alpha-enolase) & 49 & 47,7 & $-2.00 \mathrm{E}+00$ & 8.42E-02 \\
\hline & & 78 & 56,5 & $-4.50 \mathrm{E}+00$ & 5.79E-02 \\
\hline P23528 & Cofilin-1 & 72 & 14,8 & $2.60 \mathrm{E}+00$ & 2.50E-02 \\
\hline P60981 & Destrin & 73 & 14,1 & $3.90 \mathrm{E}+00$ & 5.04E-02 \\
\hline P15090 & Fatty acid binding protein. adipocyte & 68 & 10,1 & $6.50 \mathrm{E}+04$ & 6.37E-02 \\
\hline P09104 & Gamma-enolase & 10 & 48,5 & $6.40 \mathrm{E}+03$ & 7.36E-02 \\
\hline P09211 & Glutathione S-transferase P & 39 & 24,0 & $8.30 \mathrm{E}+00$ & 2.72E-02 \\
\hline P00390 & Glutathione reductase. mitochondrial & 39 & 61,3 & $4.30 \mathrm{E}+03$ & 5.11E-02 \\
\hline P04792 & Heat shock protein beta-1 & 41 & 26,6 & $1.30 \mathrm{E}+00$ & 7.28E-02 \\
\hline \multirow[t]{2}{*}{ Q6NSB4 } & HP protein * & 35 & 44,9 & $-8.70 E+04$ & 1.75E-02 \\
\hline & & 90 & 43,2 & $-5.30 E+04$ & $1.22 E-02$ \\
\hline P40926 & Malate dehydrogenase, mitochondrial & 85 & 36,6 & $3.50 \mathrm{E}+01$ & 1,35E-02 \\
\hline P30044 & Peroxiredoxin-5. mitochondrial & 70 & 13,1 & $8.90 \mathrm{E}+00$ & $9.81 \mathrm{E}-02$ \\
\hline P30086 & Phosphatidylethanolamine-binding protein 1 & 71 & 20,7 & $2.00 \mathrm{E}+00$ & 7.61E-02 \\
\hline P28074 & Proteasome subunit beta type-5 (isoform 1) & 84 & 21,5 & $2.10 \mathrm{E}+00$ & 4.21E-02 \\
\hline Q08257 & Quinone oxidoreductase & 85 & 36,6 & $3.50 \mathrm{E}+01$ & 1.35E-02 \\
\hline P04179 & Superoxide dismutase [Mn]. mitochondrial & 57 & 21.6 & $-2.30 E+00$ & 3.74E-02 \\
\hline \multirow[t]{3}{*}{ Q01995 } & Transgelin & 55 & 17.1 & $4.60 \mathrm{E}+00$ & 5.44E-02 \\
\hline & & 55 & 22.1 & $2.00 \mathrm{E}+00$ & 7.95E-02 \\
\hline & & 55 & 22.1 & $3.00 \mathrm{E}+04$ & 2.32E-04 \\
\hline P60174 & Triosephosphate isomerase (isoform 1) & 62 & 26.2 & $1.40 \mathrm{E}+04$ & $1.50 \mathrm{E}-02$ \\
\hline P15374 & Ubiquitin carboxyl-terminal hydrolase isozyme L3 * & 5 & 26.9 & $2.90 \mathrm{E}+00$ & $5.71 \mathrm{E}-02$ \\
\hline \multirow[t]{7}{*}{ P08670 } & Vimentin & 4 & 17.1 & $8.10 \mathrm{E}+00$ & $6.22 \mathrm{E}-02$ \\
\hline & & 4 & 21.1 & $6.70 \mathrm{E}+01$ & 5.32E-02 \\
\hline & & 4 & 18.6 & $1.90 \mathrm{E}+00$ & 3.27E-02 \\
\hline & & 4 & 22.1 & $2.50 \mathrm{E}+00$ & 2.04E-02 \\
\hline & & 4 & 27.2 & $4.90 \mathrm{E}+03$ & 1.99E-02 \\
\hline & & 9 & 46.1 & $-3.30 E+00$ & 2.32E-02 \\
\hline & & 11 & 44.5 & $-4.40 E+00$ & $1.88 \mathrm{E}-02$ \\
\hline
\end{tabular}


Table of Supplement 1: Identifications of secreted proteins of calorie restricted and non-calorie restricted SGBS adipocytes

\begin{tabular}{|c|c|c|c|c|c|}
\hline $\begin{array}{c}\text { accession } \\
\text { number }\end{array}$ & Protein name & ID & $\begin{array}{c}\mathrm{MW} \\
{[\mathrm{kDa}]}\end{array}$ & $\begin{array}{c}\text { fold } \\
\text { change }\end{array}$ & p-value \\
\hline \multicolumn{6}{|c|}{ non-classical secreted } \\
\hline \multirow[t]{2}{*}{ P08670 } & Vimentin & 26 & 72.7 & $-6.60 E+01$ & 6.63E-02 \\
\hline & & 76 & 42.8 & $7.10 \mathrm{E}+03$ & 3.47E-02 \\
\hline \multicolumn{6}{|c|}{ Intracellular } \\
\hline P10809 & $60 \mathrm{kDa}$ heat shock protein & 24 & 69.6 & $-3.20 E+00$ & 5.16E-03 \\
\hline \multirow[t]{2}{*}{ P60709 } & Actin & 32 & 36.3 & $-6.60 E+00$ & 1.31E-02 \\
\hline & & 35 & 44.9 & $-8.70 E+04$ & 1.75E-02 \\
\hline Q01518 & Adenylyl cyclase-associated protein 1 (Isoform 1) & 79 & 207.8 & $3.80 \mathrm{E}+00$ & 7.98E-02 \\
\hline P15121 & Aldose reductase & 59 & 36.2 & $1.40 \mathrm{E}+04$ & 2.32E-04 \\
\hline 000299 & Chloride intracellular channel protein 1 & 17 & 30.2 & $1.40 \mathrm{E}+04$ & 1.53E-02 \\
\hline P04080 & Cystatin-B & 68 & 10.1 & $6.50 \mathrm{E}+04$ & 6.37E-02 \\
\hline P00727 & Cytosol aminopeptidase (Isoform 1) & 64 & 58.8 & $-1.80 \mathrm{E}+00$ & 7.19E-02 \\
\hline P09417 & Dihydropteridine reductase & 62 & 26.2 & $1.40 \mathrm{E}+04$ & $1.50 \mathrm{E}-02$ \\
\hline Q05639 & Elongation factor 1 -alpha 2 & 69 & 13.2 & $3.10 \mathrm{E}+00$ & 7.98E-02 \\
\hline P47755 & F-actin-capping protein subunit alpha-2 & 33 & 35.6 & $-3.40 E+00$ & 8.74E-02 \\
\hline P49327 & Fatty acid synthase & 24 & 69.6 & $-3.20 E+00$ & 5.16E-03 \\
\hline Q1JUQ5 & FK506 binding protein 12 & 83 & 9.3 & $2.00 \mathrm{E}+01$ & 1.03E-02 \\
\hline P11413 & $\begin{array}{l}\text { Glucose-6-phosphate 1-dehydrogenase (Isoform } \\
\text { Long) }\end{array}$ & 65 & 61.5 & 2.10E+01 & 2.09E-02 \\
\hline P04406 & Glyceraldehyde-3-phosphate dehydrogenase & 85 & 36.6 & $3.50 \mathrm{E}+01$ & 1.35E-02 \\
\hline P11142 & Heat shock cognate $71 \mathrm{kDa}$ protein (Isoform 1) & 36 & 88.4 & $-1.70 \mathrm{E}+00$ & 9.66E-02 \\
\hline 014558 & Heat shock protein beta- 6 & 56 & 16.4 & $-4.00 E+00$ & 4.22E-02 \\
\hline \multirow[t]{2}{*}{ P40121 } & Macrophage-capping protein & 47 & 42.1 & $-1.40 \mathrm{E}+00$ & 8.39E-02 \\
\hline & & 48 & 42.2 & $6.20 E+03$ & 1.97E-03 \\
\hline P22392 & Nucleoside diphosphate kinase B (Isoform 1) & 73 & 14.1 & $3.90 \mathrm{E}+00$ & 5.04E-02 \\
\hline \multirow[t]{3}{*}{ P62937 } & Peptidyl-prolyl cis-trans isomerase A & 54 & 13.3 & $1.10 \mathrm{E}+04$ & 8.04E-02 \\
\hline & & 69 & 13.2 & $3.10 \mathrm{E}+00$ & 7.98E-02 \\
\hline & & 70 & 13.1 & $8.90 \mathrm{E}+00$ & $9.81 \mathrm{E}-02$ \\
\hline Q96Q06 & Perilipin-4 (Isoform 1) & 88 & 200 & $9.60 \mathrm{E}+02$ & 3.49E-02 \\
\hline Q96Q06 & Perilipin-4 (Isoform 2) & 89 & 200.6 & $1.60 \mathrm{E}+01$ & 7.42E-02 \\
\hline P30041 & Peroxiredoxin- 6 & 42 & 27 & $-4.40 \mathrm{E}+00$ & 8.17E-02 \\
\hline P02545 & Prelamin-A/C (Isoform A) & 42 & 27 & $-4.40 \mathrm{E}+00$ & 8.17E-02 \\
\hline \multirow[t]{2}{*}{ P07737 } & Profilin-1 & 59 & 36.2 & $1.40 \mathrm{E}+04$ & 2.32E-04 \\
\hline & & 82 & 10.4 & $1.60 \mathrm{E}+00$ & 8.26E-02 \\
\hline P06323 & Proteasome activator complex subunit 1 & 43 & 29.2 & $-6.70 E+01$ & 5.93E-06 \\
\hline P25786 & Proteasome subunit alpha type-1 (Isoform Short) & 58 & 30.7 & $-1.60 \mathrm{E}+00$ & 2.57E-03 \\
\hline \multirow[t]{2}{*}{014818} & Proteasome subunit alpha type-7 (Isoform 1) & 86 & 27.5 & $-1.40 E+00$ & 1.69E-08 \\
\hline & & 86 & 27.5 & $3.50 \mathrm{E}+03$ & 1.45E-02 \\
\hline Q2TBP0 & Proteasome subunit beta type-7 & 45 & 28.6 & $1.40 \mathrm{E}+04$ & 2.56E-02 \\
\hline Q13228 & Selenium binding protein 1 & 50 & 57.1 & $4.70 \mathrm{E}+00$ & 3.11E-02 \\
\hline P35237 & Serpin B6 & 20 & 42.3 & $-2.10 \mathrm{E}+00$ & $9.64 \mathrm{E}-02$ \\
\hline \multirow[t]{2}{*}{ P37837 } & Transaldolase & 61 & 38.3 & $7.00 \mathrm{E}+03$ & 1.19E-02 \\
\hline & & 61 & 38.3 & $7.20 \mathrm{E}+03$ & 5.53E-03 \\
\hline P55072 & Transitional endoplasmic reticulum ATPase & 26 & 72.7 & $-6.60 \mathrm{E}+01$ & 6.63E-02 \\
\hline B9A041 & Uncharacterized protein & 60 & 35.8 & $2.30 \mathrm{E}+00$ & $5.88 \mathrm{E}-02$ \\
\hline
\end{tabular}

Table of Supplement 1: Identifications of secreted proteins of calorie restricted and non-calorie restricted SGBS adipocytes by 2-DE followed by LC-MS/MS. Proteins with the identical ID number are related to the same spot and proteins marked with * are identified as novel adipocyte secreted proteins. Lines marked bolt are significant CR-stimulated proteins 
Dankwoord 


\section{Dankwoord}

In Nederland houden ze van een uitgebreid dankwoord waarin ze iedereen apart bedanken. Maar zelfs als deze dankwoorden enkele pagina's lang zijn, bestaat nog steeds het gevaar dat je iemand over het hoofd ziet en vergeet te vernoemen.

In Duitsland wordt het dankwoord dan weer vrij kort gehouden omdat ze daar niet van teveel woorden houden en zeker niemand willen vergeten of kwetsen.

Door in België te wonen en door in Nederland mijn Ph.D. te doen, heb ik geleerd dat mijn eigen cultuur een belangrijk deel van mijn persoonlijkheid uitmaakt. Mijn cultuur staat naast het gebruik van weinig woorden ook synoniem voor sociale betrekkingen en contacten. Net omwille van deze reden heb ik geen uitgebreid dankwoord geschreven en zal ik met veel plezier iedereen persoonlijk gaan bedanken die me tijdens deze periode bijgestaan en/of geholpen heeft.

Ik bedank Prof. Dr. E. Mariman en Dr. J. Renes voor het vertrouwen en voor de unieke kans om zo een inhoudelijk, praktisch en spannend project aan te mogen gaan.

De Proteomics werkgroep bestaande uit Ping, Freek en Anke bedank ik vooral voor de vriendelijke, open en plezante werksfeer. Zij waren en zijn de goede ziel van deze groep die er altijd waren als ik het moeilijk had.

Dr. Jean-Paul Noben, Erik Roymakers en de rest van het team van de Universiteit Hasselt bedank ik voor de enorme technische ondersteuning die voor het succes van de proteomics analyses en de goede resultaten van essentieel belang waren.

Mit Prof. Dr. Martin Wabitsch und Dr. Pamela Fischer-Posovszky entstand durch Ihre Bereitstellung des Zellmaterials und der offenen Diskussionsbereitschaft eine fruchtbare Zusammenarbeit, die ich sehr geschätzt habe.

Claudia en Desiree van ons administratief team, wil ik zeker niet vergeten. Zowel voor administratieve als voor persoonlijke problemen waren jullie er altijd. Heel erg bedankt voor deze steun.

Ik bedank ook de commissieleden Prof. Dr. Tilman Hackeng, Prof. Dr. Maurice van Steensel, Prof. Dr. Marleen van Baak en Prof. Dr. Frans Ramaekers voor het fungeren als commissie. Verder bedank ik ook Antoine en mijn 2 paranimfen Bianca en Marlou voor al het plezier samen en hun psychologische steun.

Zowel de commissieleden alsook de paranimfen en Desiree maakten een belangrijk deel uit van de finale punt op de "i” van mijn Ph.D.-verhaal. 
Publications 


\section{Publications}

Anja Rosenow, Jean-Paul Noben, Martin Wabitsch, Edwin C.M. Mariman, Johan Renes: Calorie restriction-induced changes in the secretome of human adipocytes, comparison with resveratrol-induced secretome effects, submitted

Anja Rosenow, Jean-Paul Noben, Freek G. Bouwman, Martin Wabitsch, Edwin C.M. Mariman, Johan Renes. (2013): Hypoxia-mimetic effects in the secretome of human preadipocytes and adipocytes. BBA - Protein and Proteomics 12:2761-2771

Rosenow A, Noben JP, Kallendrusch S, Fischer-Posovsky P, Mariman EC, Renes J. (2012): Resveratrol-induced changes of the human adipocyte secretion profile. JProtRes 11(9):473343

Rosenow A., Arrey TN. Bouwman FG., Noben JP., Wabitsch M., Mariman E., Karas M., Renes J. (2010): Identification of novel, human, adipocyte secreted proteins by using SGBS Cells. JProtRes 9: 5389-5401

Renes J., Rosenow A., Mariman E. (2009): Novel adipocyte features discovered by proteomics. Adipobiology 1: ISSN 1313-3705

Anja Rosenow, Simone J. H. Wielders, Edouard M. Bevers, Martin Wabitsch, Theo Lindhout, Edwin C.M. Mariman, Johan Renes: Expression of functional tissue factor by human (pre)adipocytes, submitted 
Curriculum Vitae 


\section{Curriculum Vitae}

Anja Rosenow was born on December 09 ${ }^{\text {th }} 1981$ in Salzwedel, Germany. She completed secondary school at the G.-E.-Lessing Realschule in 1998 and the grammar school at the Käthe Kollwitz Gymnasium in 2002 in Salzwedel. October 2002, she started her Bachelor study Biochemistry, with the specialization in organic chemistry, at Ruhr-University Bochum, Germany. For her bachelor thesis she performed a 4 month internship at the department Bioorganic Chemistry at the Ruhr-University Bochum, Germany to synthesis a 4-TEMPO-5'-O(4,4'-Dimethoxytrityl)-2'-O-(tert-butyldimethylsilyl)-uridine. She graduated as Bachelor of Science in Biochemistry September 2005. October 2005, she started her Master study Biochemistry, with the specialization in medicine and molecular biochemistry, at RuhrUniversity Bochum, Germany. To graduate as Master of Science in Biochemistry in September 2007 she performed a 9 month internship at the department of Molecular Cell Biochemistry at the Ruhr-University Bochum, Germany to express and structural characterize the adenylation domains from Ebony and U26 from Drosophila. November 2007, she started her Ph.D. study which included the investigations of novel adipokines of human adipocytes at the department of Human Biology at the Maastricht University, The Netherlands under the supervision of Prof. Dr. E. Mariman and Dr. J. Renes.

August 2012, she worked for a period of 8 month as a Scientist at Merck/MSD in the departments of analytical biochemistry and analytical development and validation at Oss, The Netherlands to develop an analytical HPLC method for the determination of protein deamidation. April 2013, she started to work as Analytical Development Specialist at Genzyme Geel, Belgium to support the chromatographic characterization group of the Technology Analytical department by project-related documentation and performance of GC, HPLC and MS analyses. In addition, she started as safety responsible person for the Technology Analytical department within Genzyme Geel. 